\title{
Control of PI4P 5-kinases by reversible phosphorylation in Arabidopsis thaliana
}

\author{
Dissertation
}

\author{
for the award of the degree \\ "Doctor rerum naturalium" \\ Division of Mathematics and Natural Sciences \\ of the Georg-August University Göttingen
}

within the doctoral program

Molecular Biology of Microbial, Animal and Plant Cells

of the Georg-August University School of Science (GAUSS)

\author{
submitted by \\ Jennifer Lerche \\ from Bad Gandersheim
}

Göttingen 2013 


\section{Mitglied der Prüfungskommission:}

Referent: $\quad$ Prof. Dr. Ingo Heilmann, Abteilung Zelluläre Biochemie, Institut für Biochemie und Biotechnologie, Martin-Luther-Universität Halle-Wittenberg

Korreferent: PD. Dr. Thomas Teichmann, Schwann-Schleiden-Forschungszentrum für Molekulare Zellbiologie, Abteilung Zellbiologie der Pflanze, Georg-AugustUniversität Göttingen

\section{Weitere Mitglieder der Prüfungskommission:}

Prof. Dr. Gregor Bucher, Johann-Friedrich-Blumenbach-Institut für Zoologie und Anthropologie, Abteilung Entwicklungsbiologie GZMB, Georg-August-Universität Göttingen

Prof. Dr. Steven Johnsen, Universitätsklinikum Hamburg-Eppendorf, Institut für Tumorbiologie Prof. Dr. Ivo Feussner, Albrecht-von-Haller-Institut GZMB, Abteilung Biochemie der Pflanze, Georg-August-Universität Göttingen

Prof. Dr. Andrea Polle, Abteilung Forstbotanik und Baumphysiologie, Institut für Forstbotanik, Georg-August-Universität Göttingen 
Herewith I affirm that I wrote this thesis independently and with no other sources and aids than quoted.

Jennifer Lerche 


\section{INDEX}

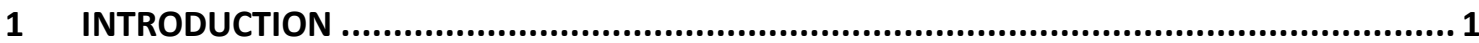

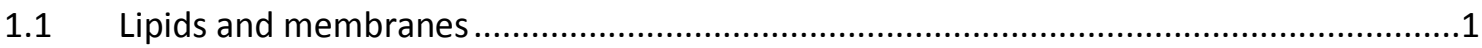





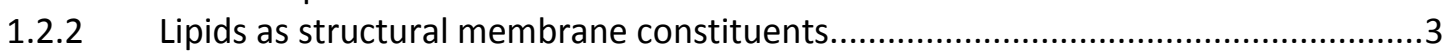

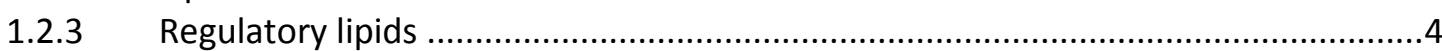

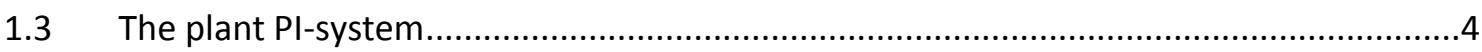

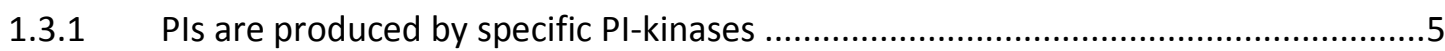

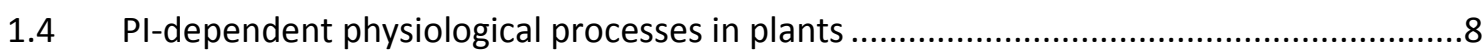

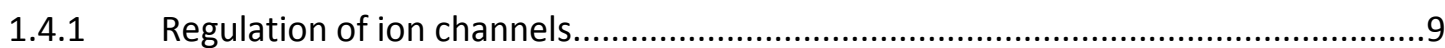

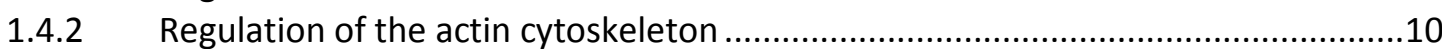

1.4.3 PIS and cellular processes controlling cell polarity ................................................11

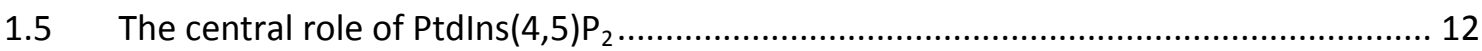

1.5.1 Ptdlns $(4,5) \mathrm{P}_{2}$-pools: How are they generated and maintained? ............................12

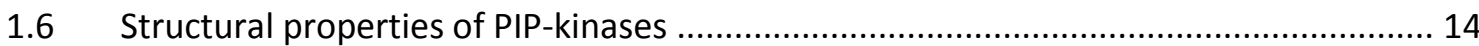

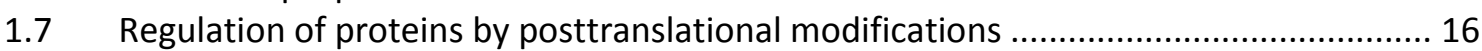

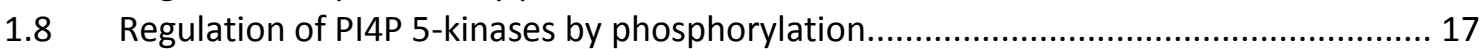

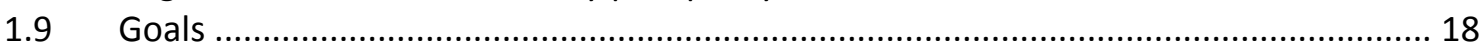

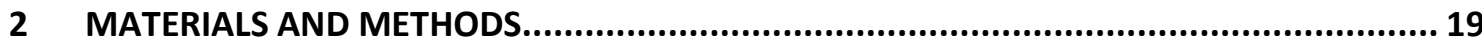

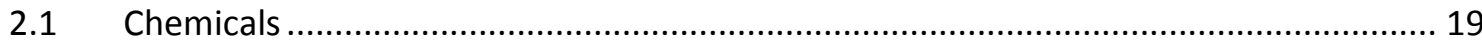

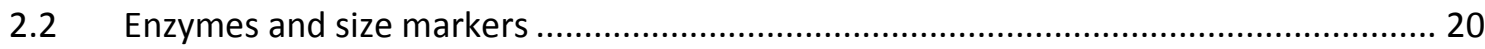

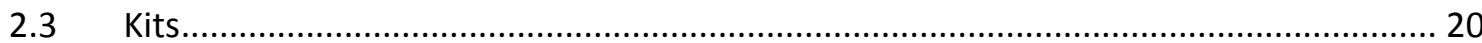

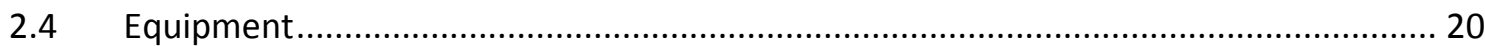



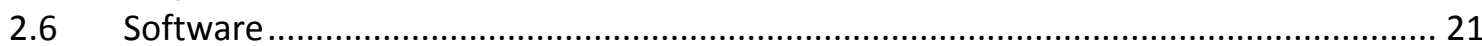

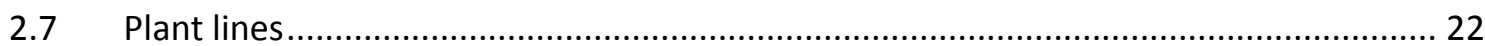

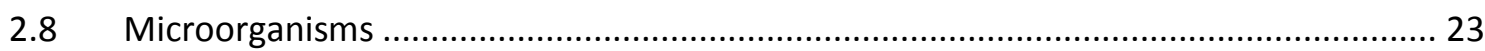

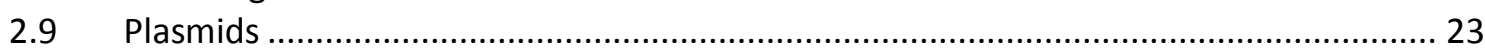

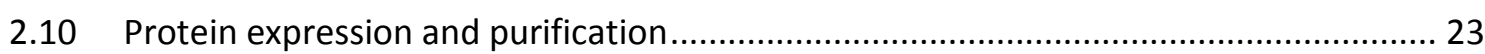

2.10.1 Preparation of chemically-competent $E$. coli cells...............................................24

2.10.2 Transformation of chemically-competent $E$. coli cells...........................................24

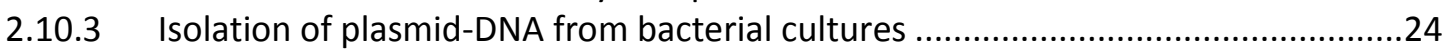

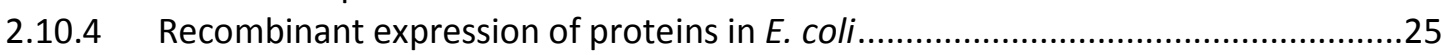

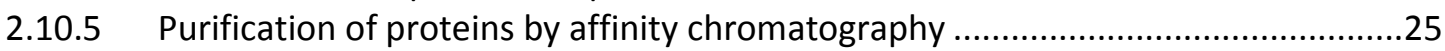



2.10.7 Separation of proteins via sodium dodecyl sulfate poly acrylamide gel

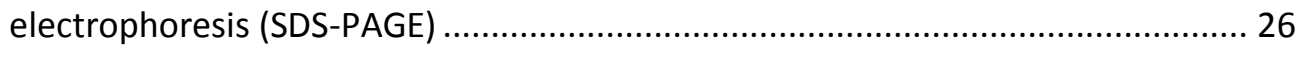

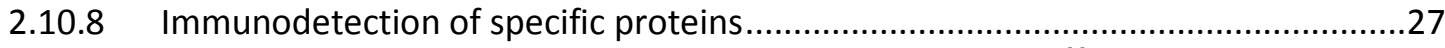

2.10.9 In vitro activity test of recombinant PI4P 5-kinases with $\mathrm{y}-\left[{ }^{32} \mathrm{P}\right]$ ATP ......................2

2.11 Detection of phosphorylation sites of in vitro-phosphorylated PIP5K2 and PIP5K3 ..... 28

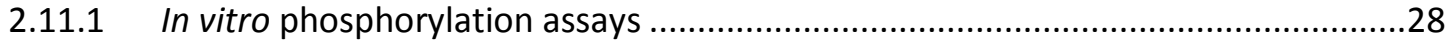

2.11.2 Tryptic in gel digestion of phosphorylated recombinant PI4P 5-kinases..................31

2.11.3 Detection of phosphorylation sites with PepStar ${ }^{\mathrm{TM}}$ peptide microarrays.................34 
2.11.4 In silico analysis of putative phosphorylation sites .35

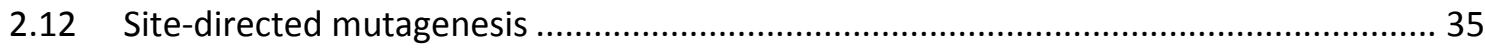

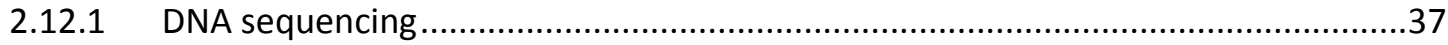

2.13 Preparation of constructs for the transformation of $A$. thaliana …............................... 37

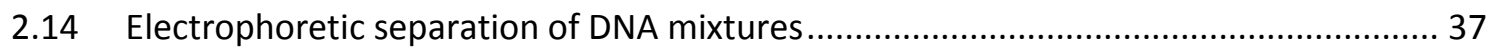

2.15 Preparation of chemically-competent Agrobacterium tumefaciens (Agrobac.



2.16 Transformation of chemically-competent Agrobac. tumefaciens cells .......................... 38



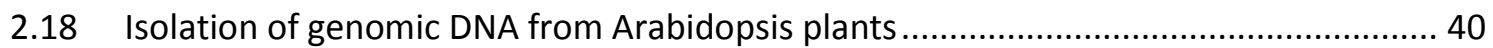



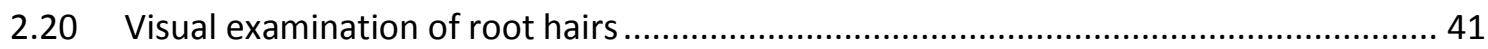

2.21 Transient expression of fluorescence-labled proteins in tobacco pollen tubes .............. 41

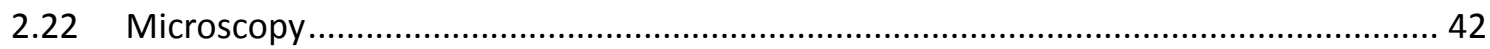

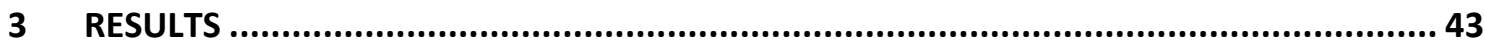

3.1 PIP5K2 can be recombinantly expressed and purified in E. coli .................................... 43

3.2 Phosphorylation of recombinantly expressed and purified PIP5K2 …........................ 45

3.2.1 Recombinant PIP5K2 is phosphorylated by PKA in vitro........................................46

3.2.2 Recombinant PIP5K2 is phoshorylated by an endogenous Arabidopsis protein

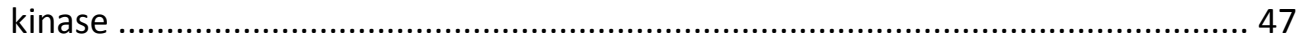

3.3 In vitro phosphorylation of recombinant PIP5K2 reduces catalytic activity ................... 49

3.4 Determination of possible phosphorylation sites in PIP5K2 ......................................... 51

3.4.1 A systematic deletion approach to delineate PIP5K2 protein regions important for phosphorylation effects on activity................................................................... 52

3.4.1.1 Phosphorylation of PIP5K2 truncations by Arabidopsis leaf extract .......................52

3.4.1.2 Effects of an Arabidopsis plant extract on catalytic activity of PIP5K2 truncations 54

3.4.2 Identification of phosphorylation sites using databases .......................................55

3.4.3 Determination of phosphorylation sites by MS ................................................57

3.4.4 Identification of phosphorylation sites by Peptide Array analysis ..........................57

3.5 Site-directed mutagenesis of selected serine and threonine residues in PIP5K2 ..........60 60

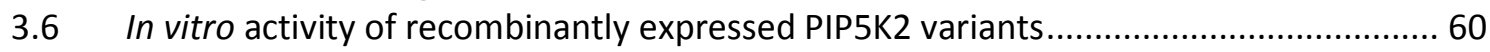

3.7 Degree of in vitro phosphorylation of PIP5K2 T430A and PIP5K2 T500A ........................62 62

3.8 Plasma membrane-association of fluorescence-tagged PIP5K2 A/D-variants transiently

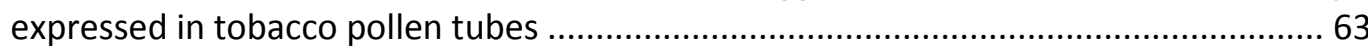

3.9 Effects of PIP5K2 A/D variants on pollen tube morphology .......................................... 64

3.10 Effects of overexpression of PIP5K2 variants in tobacco pollen tubes are not correlated

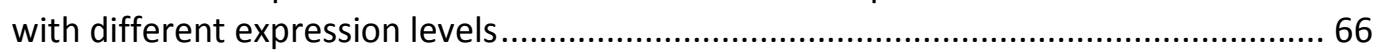



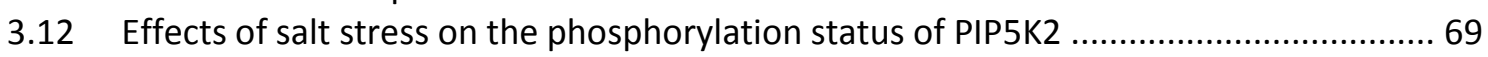

3.13 Similar or different? Control of PIP5K3 activity by reversible phosphorylation .............. 71

3.14 PIP5K3 can be recombinantly expressed in E. coli ..................................................... 71

3.15 Phosphorylation of recombinantly expressed and purified PIP5K3 ….......................... 73

3.15.1 Recombinant PIP5K3 is phosphorylated by PKA in vitro.........................................73

3.15.2 Purified PIP5K3 is phoshorylated by an endogenous Arabidopsis protein kinase ..74

3.16 In vitro phosphorylation of PIP5K3 reduces catalytic activity........................................... 76

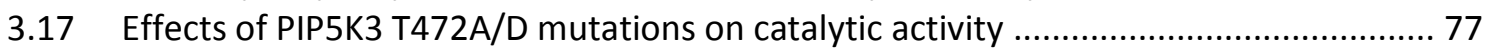


3.18 Subcellular localization of fluorescence tagged PIP5K3 T472A/D variants transiently expressed in tobacco pollen tubes ................................................................................ 79

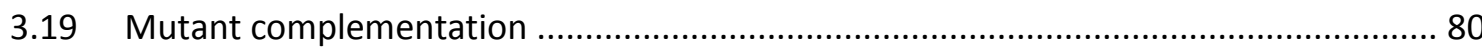



4.1 Some technical aspects of expression and enrichment of recombinant MBP PIP5K2 and MBP-PIP5K3 in E. coli 83

4.2 Similarities and differences of in vitro phosphorylation of MBP-PIP5K2 and MBP-PIP5K3 84

4.3 In vitro phosphorylation decreases catalytic activity of MBP-PIP5K2 and MBP-PIP5K3 86

$4.4 \quad \mathrm{~N}$-terminal regions of PIP5K2 might be important for its regulation ............................. 88



4.6 Phosphomimicry of some identified phosphorylation sites inhibits catalytic activity of

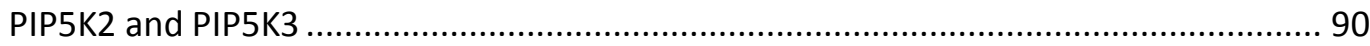

4.7 Phosphoablation of position T430 renders the MBP-PIP5K2 protein less susceptible to phosphorylation by Arabidopsis leaf extract .............................................................. 91

4.8 Are effects of phosphoablation and phosphomimicry related to altered in vivo I

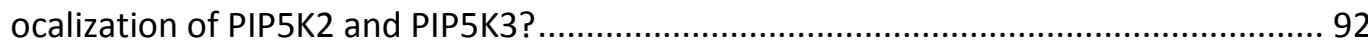

4.9 Phosphorylation of PIP5K2 and PIP5K3 in key positions is relevant for in vivo



4.10 The hypothesis of Rao et al. (1998) has to be expanded ................................................ 93

4.11 PI4P 5-kinases and their role in the response of plants to environmental stimuli......... 98

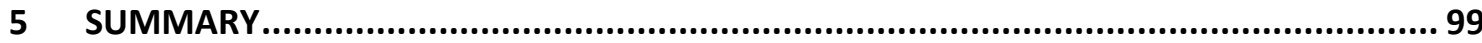

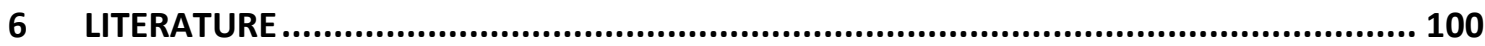

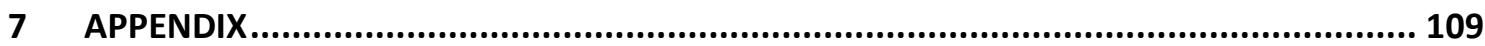

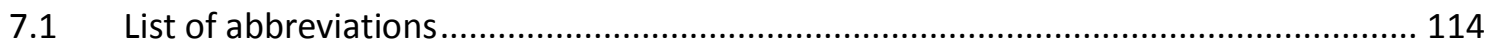

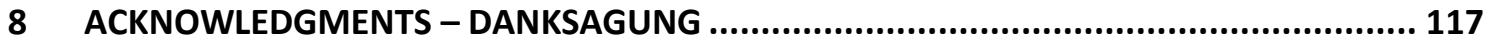






\section{INTRODUCTION}

Living cells coordinate an exceeding complexity of cellular processes, including the establishment and maintenance of cellular integrity and cellular polarity, complex metabolic reactions, movement or growth. In eukaryotes, various cellular processes are compartmentalized to enable independent functionality of contrapuntal reactions, for instance as part of anabolic or catabolic processes. Compartmentalization is the characterizing feature of eukaryotic cells, and cellular compartments are defined by membranes. In this context, phospholipids have a bi-functional role: As constituents of membranes, phospholipids aid the compartmentalization of the cell; as signaling molecules phospholipids can act as first or second messengers. The metabolic networks of a cell are not static but contain many components that dynamically adapt to developmental or environmental cues. The regulation of cellular processes targets key enzymes of a given pathway and can occur at different levels, including transcription, allosteric regulation, posttranslational modification or substrate availability. A specialized case of metabolic regulation is that exerted by signaling molecules, such as phosphoinositides (PIS), and it is the topic of this thesis to elucidate whether the dynamic formation of PIs is related to the posttranslational modification of their biosynthetic enzymes. A particular focus was on the regulation of enzymes producing the signaling lipid phosphatidylinositol 4,5-bisphosphate $\left(\operatorname{Ptd} \ln (4,5) \mathrm{P}_{2}\right)$. It is the intention of this chapter to introduce phospholipid functions in general and more specifically the regulatory functions of Pls and Ptdlns $(4,5) \mathrm{P}_{2}$. Furthermore, information will be provided on the enzymes producing $\operatorname{Ptdlns}(4,5) \mathrm{P}_{2}$ and the notion of their posttranslational modification.

\subsection{Lipids and membranes}

Phospholipids are amphipathic molecules that contain aliphatic hydrophobic carbon chains and a hydrophilic head group that can differ between lipid classes. The ensuing amphipathic character of phospholipids causes them to form lipid bilayers in aqueous solutions: hydrophobic parts will not interact with the water molecules and thus turn towards one another, whereas the hydrophilic head group of the lipid will be exposed to the aqueous solution (Alberts et al., 2002). Phospholipid bilayers are the main constituents of biological membranes and are important to the life of all cells. They not only limit the extracellular from the intracellular space but also aid maintenance of the characteristic differences between 
eukaryotic organelles, such as the nucleus, chloroplasts, mitochondria, the endoplasmic reticulum (ER) or the cytosol (Alberts et al., 2002). The compartmentalization of metabolic pathways requires the regulated exchange of energy, metabolites and information between the different organelles and also between cells and their environment (Horton et al., 2006). Composition and dynamic interaction of different membranes needs to be tightly regulated (Horton et al., 2006).

Besides phospholipids, membranes also contain embedded proteins that provide additional functional properties, such as the active transport of molecules or the enzymatic conversion of lipids, glycoproteins and polysaccharides (Singer and Nicholson, 1972). Embedded proteins and lipids are both able to move laterally in the plane of the membrane which is described in the fluid mosaic model (Fig. 1.1) and thus membrane shape can adapt to changes in intracellular status and the extracellular environment (Engelmann, 2005).

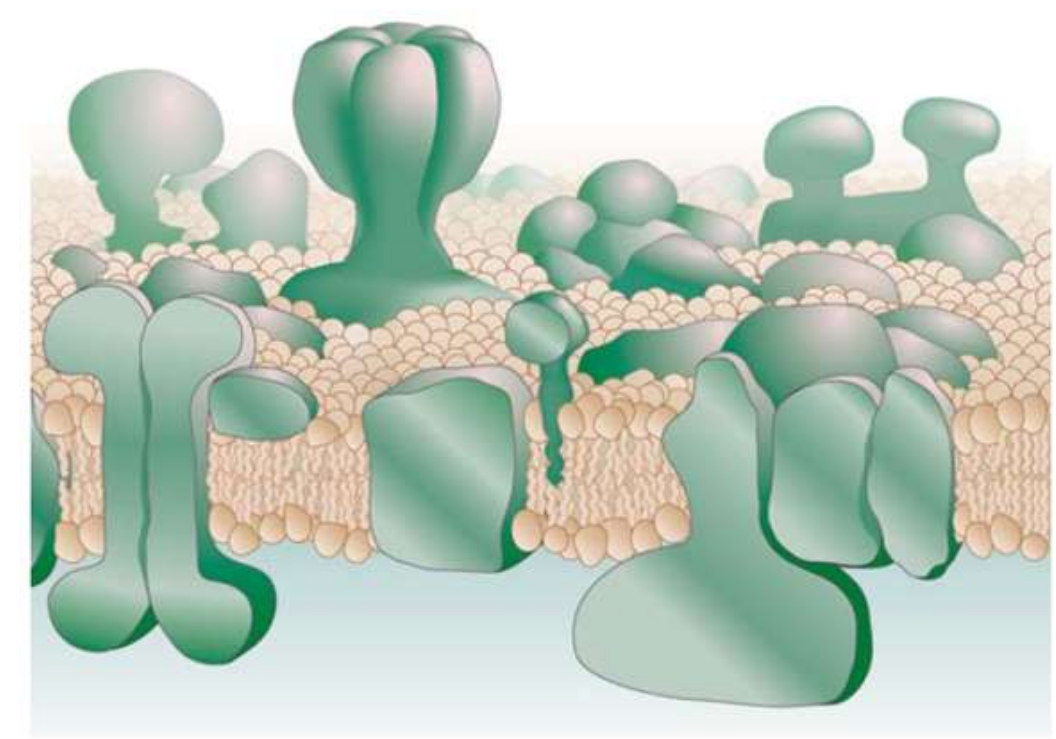

Fig. 1.1: The fluid mosaic model for the structure of cellular membranes. The major constituents of lipid bilayers are phospholipids (red) and proteins (green) that can move laterally in the plane of the membrane. From Engelmann (2005).

\subsection{Functional classes of lipids}

Lipids are hydrophobic molecules with diverse functions and can be classified into various groups: neutral lipids, including storage lipids and pigments; structural lipids, making up the body of membranes; and regulatory lipids with only low abundance but with important roles in the control of cellular processes (Somerville et al., 2000). 


\subsubsection{Neutral lipids}

Triacylglycerol (TAG) is a neutral lipid that consists of three fatty acids esterified to a glycerol backbone, resulting in an unpolar (neutral) hydrophobic molecule (Alberts, 1994). TAGs serve as an important energy depot in various organisms, including plant seeds and pollen (Somerville et al., 2000), and are the main lipids found in human food. TAGs are stored in lipid droplets also termed oil-bodies or oleosomes in the cytosol of eukaryotic organisms. In order to provide energy, TAGs are hydrolysed by lipases, releasing the fatty acids, which are then $\beta$ oxidized to yield energy and two-carbon intermediates for further metabolic conversion (Somerville et al., 2000).

Waxes are neutral lipids containing long chain unsaturated or saturated fatty acids esterified to long chain alcohols. Waxes are key components of the cuticle of most plants, which prevents desiccation, infection and ultraviolet light damage (Turkish and Sturley, 2009). Some plants like Jojoba (Simmondsia chinensis) also use oil composed of wax esters as a storage compound (Benzioni and Forti, 1989).

Pigments like Chlorophyll A and B or carotenoids also belong to the neutral lipids. These molecules derive from isoprene-units but will not be discussed further, as their structure is not related to the lipids relevant for this thesis.

\subsubsection{Lipids as structural membrane constituents}

Cellular membranes consist predominantly of structural lipids. The most abundant membrane lipids are phospholipids and glycolipids (Somerville et al., 2000). Animal cells contain an additional structural lipid, cholesterol, that is an important regulator of membrane fluidity. Phospholipids consist of four components: one or two fatty acids, a glycerol or sphingosine backbone and a primary alcohol that is bound to the backbone via a phosphodiester bond and represents the polar head group (Ohlrogge and Browse, 1995). Phospholipids that have a glycerol backbone are called glycerophopsholipids (Somerville et al., 2000). The simplest glycerophospholipid is phosphatidic acid (PtdOH) (Ohlrogge and Browse, 1995) that contains a phosphate group as a polar head group. All other glycerophospholipids are derivatives of $\mathrm{PtdOH}$ and can contain alcohols like choline, ethanolamine, serine or inositol as polar head groups (Ohlrogge and Browse, 1995). Glycerophospholipids with important structural properties are phosphatidylcholine (PtdCho), phosphatidylethanolamine (PtdEtn) and phosphatidylserine (PtdSer). Structures of PtdEtn and PtdCho are shown in Figure 1.2. 




Fig. 1.2: Structure of important structural glycerophospholipids. Gylcerophospholipids consist of a glycerin backbone that is esterified to two fatty acids ( $R$ and $\left.R^{\prime}\right)$, and an alcohol headgroup assoziated with the glycerol backbone via a phosphodiester bond. The head groups of PtdEtn and PtdCho are phosphoethanolamine and phosphocholine, respectively.

Glycoglycerolipids are the main contents of the thylakoid membranes in plastids (Ohlrogge and Browse, 1995). Like glycerophospholipids, glycoglycerolipids contain a glycerine backbone and two esterified fatty acids. The head group of the glycolipids consists of mono- or oligosaccharides (Curatolo, 1987). Important representatives are monogalactosyldiaclyglycerol (MGDG) and digalactosyldiacylglycerol (DGDG) (Somerville et al., 2000)

Sphingolipids form another class of structural lipids. They contain the long-chain aminoalcohol, sphingosine, or a derivative as a backbone (Sperling and Heinz, 2003), which is associated with a fatty acid via an amide linkage. The structure is then called ceramide. Ceramides can associate with different polar head groups, including phosphocholine or mono- and oligosaccharides and are substantial contents of membranes (Sperling and Heinz, 2003). In the context of this thesis glycolipids and sphingolipids will not be further discussed.

\subsubsection{Regulatory lipids}

Although the majority of phospholipids are membrane components with essential structural characteristics there is a class of phospholipids with only low abundance that has regulatory functions (Munnik et al., 1998; Stevenson et al., 2000; Heilmann, 2009). Phosphoinositides (PIs) belong to this group of regulatory lipids and are present in eukaryotic cells at only $1-3$ mol \% of the total phospholipids (Stevenson et al., 2000; Balla, 2006). Since PIs are the main focus of this thesis their structure and their synthesis will be described in more detail in the following sections.

\subsection{The plant PI-system}

PIs are glycerophospholipids and derive from the structural phospholipid, phosphatidylinositol (PtdIns). The D-myo-inositol head group of Ptdlns can be phosphorylated at positions 3, 4 and/or 5 (Stevenson et al., 2000; Balla, 2006). The sequential phosphorylation of the inositol 
head group gives rise to seven different PI-derivatives all having characteristic functions: phosphatidylinositol-monophosphate (Ptdlns3P, Ptdlns4P, Ptdlns5P), phosphatidylinositolbisphosphate $\left(\operatorname{Ptd} \operatorname{lns}(3,4) \mathrm{P}_{2}, \operatorname{Ptd} \operatorname{lns}(3,5) \mathrm{P}_{2}, \operatorname{Ptd} \ln s(4,5) \mathrm{P}_{2}\right)$ and phosphatidylinositol-3,4,5trisphosphate (Ptdlns(3,4,5) $\mathrm{P}_{3}$ ) (Fig. 1.3). Phosphorylation at position 2 and 6 of the inositol head group is sterically unfavorable. Although the PI-monophosphates and PI-bisphosphates are generated in all eukaryotic organisms (Anderson et al., 1999; Drobak et al., 1999), so far there is no evidence that PtdIns $(3,4,5) P_{3}$ is present in any plant model (Munnik et al., 1998; Stevenson et al., 2000). The structures of plant PIs are illustrated in Figure 1.3.
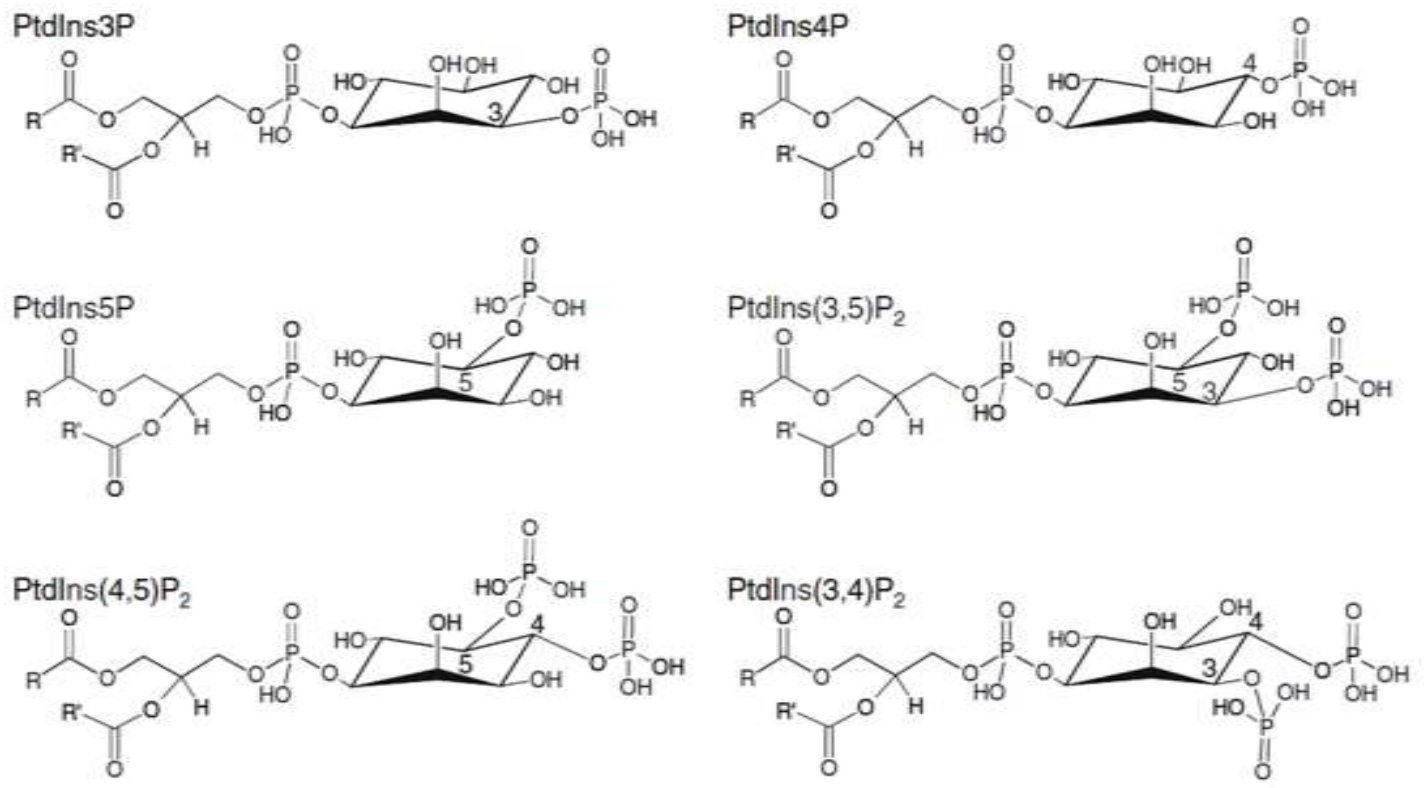

Fig. 1.3: Structure of PIs present in plants. PIs are glycerophospholipids containing an inositol head group. The inositol ring can be phosphorylated at particular positions giving rise to six known Pls in plants: Ptdlns3P, phosphatidylinositol-3-phosphate. Ptdlns4P, phosphatidylinositol-4-phosphate. Ptdlns5P, phosphatidylinositol-5-phosphate. Ptdlns $(3,5) \mathrm{P}_{2}$, phosphatidylinositol-3,5-bisphosphate. Ptdlns $(4,5) \mathrm{P}_{2}$, phosphatidylinositol-4,5-bisphosphate. Ptdlns $(3,4) \mathrm{P}_{2}$, phosphatidylinositol-3,4bisphosphate. Figure from (Ischebeck and Heilmann, 2010).

For the functional analysis of the plant PI-system it is necessary to consider the enzymes producing the various PIs (Mueller-Roeber and Pical, 2002). This knowledge can then aid in influencing the PI-metabolism by genetical or pharmacological approaches. Plant enzymes producing PIs are the focus of the next section.

\subsubsection{Pls are produced by specific PI-kinases}

The precursor of all PIs, PtdIns, is generated in the cytosolic leaflet of the ER membrane by the action of Ptdlns-synthases (PIS), which condensate cytidine-diphosphodiacylglycerol (CDPDAG) with D-myo-inositol (Justin et al., 1995; Ohlrogge and Browse, 1995; Jackson et al., 2000). Arabidopsis thaliana (A. thaliana) contains two PIS isoforms (PIS1 and PIS2) with high sequence 
similarity and both isoforms are localized in the ER (Löfke et al., 2008). Ptdlns is dispersed to various cellular membranes by a so far unknown mechanism. It is suggested that Ptdlns is relocalized by direct vesicle trafficking or by the activity of lipid transfer proteins (Vincent et al., 2005; Phillips et al., 2006). A combination of both mechanisms is also possible (Heilmann, 2008).

PtdIns-monophosphates are synthesized by phosphorylating the D-3 or D-4 position of the inositol head group, giving rise to Ptdlns3P or Ptdlns4P, respectively. The production of Ptdlns3P is catalyzed by phosphoinositide 3-kinases (PI3Ks). In plants and fungi PI3Ks belong to the class III PI3-kinases and only accept PtdIns as a substrate (Mueller-Roeber and Pical, 2002). PI3Ks of the classes I and II are able to phosphorylate additional PIs. Although both classes play an important role in animals (Vanhaesebroeck et al., 2001) they have not been reported for plants yet.

Ptdlns4P is produced by the action of phosphoinositide 4-kinases (PI4Ks) that have been found in animals (Endemann et al., 1987; Pike, 1992)and in plants (Okpodu et al., 1995; Stevenson et al., 1998; Pical et al., 1999; Xue et al., 1999). In Arabidopsis there are four ubiquitously expressed PI4Ks (Mueller-Roeber and Pical, 2002; Schmid et al., 2004) which are soluble proteins that peripherally localize to membranes. Localization of plant PI4-kinases has been analyzed in Arabidopsis root hairs (Preuss et al., 2006) and upon heterologous expression in insect cells (Stevenson-Paulik et al., 2003) and found at trans-Golgi vesicles and other, so far unidentified subcellular locations. So far, the biosynthesis of Ptdins5P is not clear and no Ptdlns kinase capable of phosphorylating the D-5 position has been found encoded in a plant genome (Heilmann, 2009). Ptdlns5P formation might involve the production of a 5phosphorylated Ptdlns-bisphosphate and subsequent dephosphorylation by an unknown phosphatase (Heilmann, 2009) .

The production of PtdIns-bisphosphates is catalyzed by PtdlnsP-kinases (PIPKs), which have been categorized in three classes (type I - III), depending on their substrate specificity (Hinchliffe et al., 1998; Mueller-Roeber and Pical, 2002). Type I PIPKs, which are the major focus of this thesis, can phosphorylate Ptdlns4P and Ptdlns3P (Mueller-Roeber and Pical, 2002). Type I PIPKs from Arabidopsis have a preference to phosphorylate Ptdlns4P (Elge et al., 2001; Perera et al., 2005; Lee et al., 2007; Kusano et al., 2008; Stenzel et al., 2008) and are, therefore, also referred to as PI4P 5-kinases. The Arabidopsis genome encodes eleven type I PI4P 5-kinase isoforms (Mueller-Roeber and Pical, 2002). Based on their domain structure, these isoforms have been grouped into the subfamilies A (isoforms 10 and 11 ) and B (isoforms 1 - 9) (Mueller-Roeber and Pical, 2002). As illustrated in Figure 1.4, PI4P 5-kinases of subfamily B subfamily are larger $(81-92 \mathrm{kDa})$ than those of subfamily A (46-48 kDa) due to the 
presence of additional $\mathrm{N}$-terminal domains. PI4P 5-kinases of subfamily B contain a characteristic membrane occupation and recognition nexus (MORN)- domain (Mueller-Roeber and Pical, 2002) with putative functions in plasma membrane binding (Takeshima et al., 2000; Im et al., 2007). The NT- and MORN-domains are joined to the rest of the protein by a linker (Lin)-domain. While originally no particular in vivo functionality was attributed to the Lindomain, recent evidence indicates that the Lin-domain directs PI4P 5-kinases into relevant regulatory contexts (Stenzel et al., 2012). All other domains are also present in subfamily A PI4P 5-kinases and thus resemble PIPKs from animal systems.

\section{Subfamily A \\ Subfamily B

\begin{tabular}{|c|c|}
\hline एшा & MORN \\
\hline
\end{tabular}

\section{Cat}

Fig. 1.4: Domain structure of PI4P 5-kinases from Arabidopsis. In Arabidopsis PI4P 5-kinases are grouped into subfamilies A and B. PI4P 5-kinases of subfamily B are larger and contain additional Nterminal domains not present in the smaller enzymes of subfamily A. NT, N-terminal domain. MORN, membrane occupation and recognition nexus-domain. Lin, Linker domain. Dim, Dimerization domain. Cat, Catalytic domain.

Experimental evidence (Lou et al., 2007; Stenzel et al., 2008) and Genevestigator analysis (Zimmermann et al., 2004) revealed that six of the Arabidopsis PI4P 5-kinase isoforms (AtPIP5K1, AtPIP5K2, AtPIP5K6, AtPIP5K7, AtPIP5K8 and AtPIP5K9) are ubiquitously expressed, whereas four isoforms are predominantly expressed in pollen tubes (AtPIP5K10, AtPIP5K11, AtPIP5K4, AtPIP5K5) (Schmid et al., 2004; Ischebeck et al., 2008; Sousa et al., 2008; Zhao et al., 2010; Ischebeck et al., 2011). One isoform, AtPIP5K3, is mainly expressed in the root cortex and in root epidermal cells (Kusano et al., 2008; Stenzel et al., 2008). With regard to their subcellular distribution, diverse reports indicate that PI4P 5-kinases are soluble proteins which can localize at the plasma membrane of animal cells, yeast and of Arabidopsis (Rao et al., 1998; Lee et al., 2007; Ischebeck et al., 2008; Kusano et al., 2008; Stenzel et al., 2008). Additionally PI4P 5-kinase activity has been found in other subcellular fractions like the actin cytoskeleton of yeast and plants (Desrivieres et al., 1998; Doughman et al., 2003; Davis et al., 2007), nuclei of mammals and yeast (Ciruela et al., 2000; Audhya and Emr, 2003; Santarius et al., 2006) and in endomembranes of animal and plant cells (Whatmore et al., 1996; Heilmann et al., 1999; Heilmann et al., 2001; Im et al., 2007). To date, the mechanisms directing PI4P 5-kinases to their target membranes in a cell are not clear.

The type II PIPKs are PI5P 4-kinases and thus catalyze the phosphorylation of PtdIns5P to form PtdIns(4,5) $\mathrm{P}_{2}$ (Clarke et al., 2007). No PI5P 4-kinase has been identified in plants (MuellerRoeber and Pical, 2002). The Type III PIPKs synthesize Ptdlns $(3,5) \mathrm{P}_{2}$ by phosphorylating 
Ptdlns3P and are called PI3P 5-kinases. Although the Arabidopsis genome encodes four PI3P 5kinase genes the corresponding gene products have not been shown to be active enzymes or further characterized (Mueller-Roeber and Pical, 2002; Ischebeck et al., 2010).

Since the PI network regulates diverse physiological processes in eukaryotic organisms the termination of PI signaling is equally important as signal generation (Berdy et al., 2001; Ercetin and Gillaspy, 2004). Organisms have evolved several mechanisms to turn off PI signaling, for instance by the dephosphorylation of the inositol polyphosphate head group. In Arabidopsis there are several genes encoding for putative phosphoinositide phosphatases. The SAC (suppressor of actin) family consists of nine genes encoding phosphatases that dephosphorylate Ptdlns4P and PtdIns $(3,5) \mathrm{P}_{2}$. SAC phosphatases have high similarity to Sac1p (Hughes et al., 2000; Foti et al., 2001) and Fig4P (Rudge et al., 2004) from yeast. Other phosphatases from Arabidopsis have similarity to the PTEN (phosphatase and tensin homolog) family from mammals (Gupta et al., 2002).

Another way to terminate $\mathrm{PI}$ signals is the specific hydrolysis of the PI molecule by phospholipases. PI-specific phospholipase C (PI-PLC) hydrolyses several PIs by cleaving the soluble inositol polyphosphate head group from the DAG backbone (Berridge and Irvine, 1984). The hydrolysis of Ptdlns $(4,5) \mathrm{P}_{2}$, thus, releases $\operatorname{lns} \mathrm{P}_{3}$ that can then influence additional signaling cascades. In Arabidopsis there is one family of PI-PLCs that contains 7 isoforms. The isoforms share high similarity with mammalian type $\zeta$ PLCs and need $\mathrm{Ca}^{2+}$ for activity (MuellerRoeber and Pical, 2002). Plant PI-PLCs are located to the plasma membrane (Melin et al., 1992; Dowd et al., 2006).

Although the PI-system is conserved between eukaryotic kingdoms and physiological functions of PIs are well investigated in mammals and yeast (Balla, 2006; Strahl and Thorner, 2007), there is still little known about the PI-signaling in plants. The next section will therefore highlight important physiological processes regulated by PIs in the plant system.

\subsection{PI-dependent physiological processes in plants}

PIs can perform cellular functions in plant and animal cells either as intact lipids acting as ligands for protein partners (Drobak et al., 1994; Lemmon, 2003; Balla, 2005) or by being a substrate for phospholipase C (PLC). PLC hydrolyzes Ptdlns $(4,5) \mathrm{P}_{2}$ into DAG and InsP $\mathrm{P}_{3}$ (Berridge, 1983) which can then activate additional signalling cascades.

Known functions of intact PIs are the activation of ion channels and ATPases (Varsanyi et al., 1983; Memon and Boss, 1990; Starling et al., 1995), the regulation of stomatal opening (Lee et al., 1996) or the regulation of the actin cytoskeleton (Lassing and Lindberg, 1985; Doughman et 
al., 2003; Hilpela et al., 2004). Additionally, various biotic or abiotic stresses result in changing Ptdlns $(4,5) \mathrm{P}_{2}$-levels which has been shown for all eukaryotic systems so far and suggests a role for PIs in stress signalling (Mueller-Roeber and Pical, 2002; Balla, 2006; Heilmann, 2009). Previous studies on the involvement of PIs in polar tip growth of pollen tubes or root hairs strengthen the central role for Pls in plant function and development (Heilmann, 2009; Ischebeck and Heilmann, 2010). To emphasize the complexity of PI signalling, some functions will be described in further detail in the next sections.

\subsubsection{Regulation of ion channels}

Although lipid bilayers are highly impermeable to most polar molecules, the exchange of substances between the intra- and the extracellular space is important for living organisms. Cells have evolved several transport mechanisms to relocate ionic molecules across the membrane, such as carrier proteins or ion channels and all of these mechanisms are highly selective for particular ions. The activity of ion channels is regulated by several factors, including by PIs. It has been suggested that ion channels bind Ptdlns $(4,5) \mathrm{P}_{2}$ via specific $\mathrm{N}$ and/or C-terminal binding domains (Suh and Hille, 2008). The first ion channels found to be regulated by Ptdlns $(4,5) \mathrm{P}_{2}$ were the mammalian inward-rectifier $\mathrm{K}^{+}$-channels (Hilgemann and Ball, 1996). These channels are activated by binding $\operatorname{Ptd} \ln s(4,5) \mathrm{P}_{2}$ with six basic residues located at the C-terminal cytosolic domain (Haider et al., 2007). An equivalent mechanism has been proposed for mammalian voltage-gated $\mathrm{K}^{+}$-channels (Delmas and Brown, 2005; Li et al., 2005). The transient receptor potential (TRP) channels are an interesting example for the different consequences Ptdlns(4,5) $\mathrm{P}_{2}$ binding can have, because some representatives like TRPV5 are activated upon PtdIns $(4,5) \mathrm{P}_{2}$ binding (Rohacs et al., 2005) whereas others like TRPLs are inhibited by Ptdlns $(4,5) \mathrm{P}_{2}$ (Estacion et al., 2001). In plants ion channel regulation by $\operatorname{Ptdln}(4,5) \mathrm{P}_{2}$ is less well studied. It was proposed that plant shaker-type $\mathrm{K}^{+}$-channels are activated by Ptdlns(4,5) $\mathrm{P}_{2}$ (Liu et al., 2005; Ma et al., 2009). Furthermore it has been suggested that $\mathrm{Ptd} \operatorname{lns}(4,5) \mathrm{P}_{2}$ inactivates slow anion channels involved in stomatal opening (Lee et al., 2007).

Stomata are located in epidermis cells of leaves and stems of plants and control the exchange of gas and water during photosynthesis and transpiration, respectively (Hetherington, 2001). During photosynthesis $\mathrm{CO}_{2}$ is assimilated to form energy-rich carbon compounds in the plastids. At the same time, $\mathrm{O}_{2}$ originating from the cleavage of water needs to be transported out of the leaf tissue to avoid oxidative toxicity. The regulation of stomatal opening is attended by changes of turgor pressure in the guard cells (Gilroy et al., 1990). Stomata are stimulated by 
various environmental influences including light or $\mathrm{CO}_{2}$, that often result in changes in cytosolic $\mathrm{Ca}^{2+}$-levels. InsP $\mathrm{P}_{3}$ and $\mathrm{Ca}^{2+}$ have both been reported to induce the closing of stomata (Gilroy et al., 1990). Additionally it has been suggested that the phytohormone abcisic acid (ABA) acts on stomatal closure by increasing $\mathrm{Ca}^{2+}$-levels (Hetherington, 2001). This effect is possibly caused by decreases in the levels of Ptdlns4P and Ptdlns $(4,5) \mathrm{P}_{2}$, and a simultaneous increase of InsP $\mathrm{P}_{3}$ (Lee et al., 1996). In line with these observations, exogenous application of PtdIns $(4,5) \mathrm{P}_{2}$ activates the opening of stomata (Lee and Miller, 2007). Interestingly, application of other PIs proved ineffective (Lee and Miller, 2007). Although a number of independent observations have been made in the last years, the mechanisms activating stomata opening upon Ptdlns $(4,5) \mathrm{P}_{2}$ action remain unclear. The changes in guard cell shape responsible for stomatal movement are in part related to dynamic alterations in the actin cytoskeleton. Among cellular processes influenced by Pls, reported effects on the actin cytoskeleton are very important and might touch on various aspects of physiology.

\subsubsection{Regulation of the actin cytoskeleton}

The actin cytoskeleton is a highly dynamic network of protein filaments that not only contributes to cell shape and structure but also plays a role in vesicle- and protein- trafficking, cell division and signalling (Wasteneys and Galway, 2003).

The cytoskeleton of eukaryotic cells consists of three types of filaments. Microtubules are filamentous, hollow cylinders of about $25 \mathrm{~nm}$ in width and consist of $\alpha$ and $\beta$ tubulin-units. Actin filaments are the thinnest components of the cytoskeleton and thus are also called microfilaments. They are about 5-9 $\mathrm{nm}$ in diameter, very flexible and consist of globular actin subunits (G-actin) that can bind ATP or ADP. F-actin is a polar structure, with a barbed (+) and a pointed (-) end. In the presence of ATP actin polymerizes spontaneously by adding G-actin monomers to the barbed or to the pointed end. This process is regulated in vivo by the action of actin binding proteins (ABPs) (Wasteneys and Galway, 2003). Some ABPs found in the animal system have homologues in plants (Wasteneys and Yang, 2004) while other animal AGPs are completely missing in the plant genome, like talin, vitronectin or vinculin (Wasteneys and Galway, 2003). The intermediate filaments of animal and fungal cells provide mechanical strength and are composed of a heterogenous set of proteins associating to fibers of around $10 \mathrm{~nm}$ thickness. Intermediate filaments have not been reported for plants (Wasteneys and Galway, 2003).

Although the organization and functionality of the cytoskeleton of plants and animals is clearly different, the regulation of actin dynamics by PIs seems to have been conserved in evolution 
between animals and plants. In this respect, Pls do not directly bind to actin but seem to regulate some of the ABPs (Wasteneys and Galway, 2003).

ABPs of the ADF (actin-depolarization factor)/cofilin-family destabilize the actin filament by aiding the degradation of the pointed end (Bailly et al., 2001) and it has been shown that the ADF/cofilin proteins are inhibited by PtdIns(4,5) $\mathrm{P}_{2}$ (Ojala et al., 2001). In line with this concept it has been observed that membranes rich in Ptdlns $(4,5) \mathrm{P}_{2}$ stabilize actin filaments (Yin and Janmey, 2003). A similar regulatory mechanism has been postulated for plants (Gungabissoon et al., 1998).

Profilins are small ABPs that bind monomeric G-actin and can have positive or negative effects on F-actin assembly in vitro. Profilins can bind PtdIns $(4,5) \mathrm{P}_{2}$ (Sohn et al., 1995; Kovar et al., 2000) and this binding causes profilin to localize to the plasma membrane resulting in the release of G-actin monomers (Witke, 2004). When Ptdlns $(4,5) P_{2}$ is hydrolysed by PLC, profilin is released and can regulate F-actin again. In plants, profilins play an important role in cell elongation and cell shape of sporophytes (Ramachandran et al., 2000). Similar to some PI4P 5kinases, profilins have been shown to localise to the apical plasma membrane of growing root hairs (Braun et al., 1999).

The manipulation of the PI content in living cells has been shown to have severe effects on the cytoskeleton. For instance, a decrease in the Ptdlns $(4,5) \mathrm{P}_{2}$-content in animal fibroblasts, results in reduced attachment of the cytoskeleton to the plasma membrane (Raucher et al., 2000). Reciprocally, increased of Ptdlns $(4,5) \mathrm{P}_{2}$-levels has been shown to result in the aggregation and hyperpolimerization of the actin cytoskeleton of mammalian cell lines (Shibasaki et al., 1997; Rozelle et al., 2000; Yamamoto et al., 2001). Recent investigations on tobacco pollen tubes suggest an influence of Ptdlns $(4,5) \mathrm{P}_{2}$ on actin cytoskeleton dynamics also in plants (Ischebeck et al., 2011). In the context of these studies complex effects of PIs on the establishment of cell polarity were described.

\subsubsection{PIS and cellular processes controlling cell polarity}

An important characteristic of multicellular organisms is their polarity, which plays central roles in dictating the patterns of cell division, organogenesis and growth. Polarity can be found at every developmental level and is not restricted to complex organisms, as even single cells display polarity in the orientation of the Golgi apparatus or the patterns of cytoplasmic streaming. Extreme examples of single cells exhibiting polar growth are fungal hyphae, neurons, root hairs or pollen tubes which all show extreme forms of polar tip-growth. The regulation of the cellular processes underlying tip-growth is connected to $\operatorname{Ptd} \ln s(4,5) \mathrm{P}_{2}$ (Thole 
et al., 2008).In fungal hyphae, growing pollen tubes and root hairs Ptdlns $(4,5) P_{2}$ is localized at the apical plasma membrane and has been shown to be essential for growth (Mahs et al., 2012; Braun et al., 1999; Kost et al., 1999; Ischebeck et al., 2008; Kusano et al., 2008; Sousa et al., 2008; Stenzel et al., 2008). In Arabidopsis, lack of AtPIP5K3 resulted in compromised root hair formation, whereas overexpression of AtPIP5K3 resulted in non-polar spherical growth of root hairs (Stenzel et al., 2008). A corresponding swelling phenotype was observed in petunia pollen tubes upon inhibition of PLC-activity and thus leading to an increased level of PtdIns (4,5) $\mathrm{P}_{2}$ (Dowd et al., 2006) and in pollen tubes overexpressing certain PI4P 5-kinase isoforms (Ischebeck et al., 2011; Stenzel et al., 2012).

\subsection{The central role of PtdIns $(4,5) \mathrm{P}_{2}$}

Ptdlns(4,5) $\mathrm{P}_{2}$ is the best-studied regulatory PI (Chen et al., 1991; Munnik et al., 1998; Stevenson et al., 2000) and plays important regulatory roles in both animal and plant cells by controlling a diverse set of processes. Since it is obvious that Ptdlns $(4,5) \mathrm{P}_{2}$ can bind and thus regulate more than one alternative protein partner, it has been suggested that different functions of Ptdlns $(4,5) \mathrm{P}_{2}$ are orchestrated by the establishment of distinct subcellular pools (Doughman et al., 2003; Santarius et al., 2006). How Ptdlns(4,5) $\mathrm{P}_{2}$-pools with different metabolic origin and/or different spatio-temporal localization (Doughman et al., 2003; Santarius et al., 2006) are established and maintained is yet not well understood. Some concepts will be presented in the following paragraphs.

\subsubsection{Ptdlns $(4,5) \mathrm{P}_{2}$-pools: How are they generated and maintained?}

As described in the last sections there are several functions reported for $\operatorname{Ptdlns}(4,5) \mathrm{P}_{2}$ (Santarius et al., 2006; Meijer and Munnik, 2003; Mueller-Roeber and Pical, 2002; Stevenson et al., 2000), suggesting that the organization of $\operatorname{Ptd} \operatorname{lns}(4,5) \mathrm{P}_{2}$ in distinct physiological pools can be compartmentalized along organellar borders or in membrane microdomains in plants and animals (King et al., 1987; Liu et al., 1998; Heilmann et al., 1999; Kost, 2008; König et al., 2007). Since the production of PIs is accomplished by the action of several enzymes often present in multiple isoforms it is possible that particular Ptdlns $(4,5) \mathrm{P}_{2}$-pools are generated by particular isoforms associated with these pools. For PISs it has been reported that the isoforms PIS1 and PIS2 have different preferences for CDP-DAG-species containing unsaturated or saturated acyl chains (Löfke et al., 2008). Furthermore, the suborganellar localization of PIS1 and PIS2 at the ER is not strictly identical and it has been proposed that the Ptdlns synthesized is channeled 
into different metabolic pathways (Löfke et al., 2008). From animal models it is known that PIkinases and phosphatases can have preferences for PI-substrates with distinct fatty acid compositions (Carricaburu and Fournier, 2001; Schmid et al., 2004). In plants it has previously been observed that the fatty acid composition of $\operatorname{Ptdlns}(4,5) \mathrm{P}_{2}$ generated after application of hyperosmotic stress differs from that present before the stress (König et al., 2007), indicating that distinct Ptdlns $(4,5) \mathrm{P}_{2}$-species may function in different physiological contexts. In tobacco pollen tubes it has been shown overexpression of different isoforms of PI4P 5-kinases resulted in exclusive effects on different cellular processes (Ischebeck et al., 2008; Sousa et al., 2008). In subsequent studies, it has been demonstrated that the alternative recruitment of PI4P 5kinases to different microdomains within the plasma membrane of growing pollen tubes will direct Ptdlns $(4,5) \mathrm{P}_{2}$ toward different physiological effects (Stenzel et al., 2012).

The concepts described for the establishment of cellular PtdIns $(4,5) \mathrm{P}_{2}$-pools rely on the spatial separation of PI4P 5-kinase isoforms with unique functions in different compartments or even within microdomains of the same membrane. An important additional aspect possibly contributing to the formation of distinct PtdIns $(4,5) \mathrm{P}_{2}$-pools is the regulation of $\operatorname{Ptdlns}(4,5) \mathrm{P}_{2}$ production via the dynamic regulation of PI4P 5-kinases, that might specifically be inhibited or activated according to cellular requirements. There are various examples for stress-induced activation of enzymes synthesizing or hydrolyzing Ptdlns $(4,5) \mathrm{P}_{2}$ (Stevenson et al., 2000; Meijer and Munnik, 2003). In the red alga Galdieria sulphuraria it was found that plasma membraneassociated PI4P 5-kinase activity is induced within seconds after application of hyperosmotic stress (Heilmann et al., 1999; Heilmann et al., 2001). Similarly, gravitropic stimulation of maize stems resulted in transient increases in plasma membrane-associated PI4P 5-kinase activity within seconds of stimulation (Perera et al., 1999). Furthermore, subjecting Arabidopsis cell cultures or intact Arabidopsis plants to hyperosmotic stress led to increased Ptdlns $(4,5) \mathrm{P}_{2}$ synthesis (Pical et al., 1999; DeWald et al., 2001; König et al., 2007). The rapid nature of Ptdlns $(4,5) P_{2}$ production within seconds of exposure to stress suggests the involvement of posttranslational modification rather than transcriptional regulation (Heilmann et al., 1999; Pical et al., 1999; König et al., 2007). Since the elucidation of PI4P 5-kinase regulation has an important impact on further understanding the plant PI network, it was the main goal of this thesis to elucidate whether and how plant PI4P 5-kinases are regulated by posttranslational modifications, in particular reversible phosphorylation. Before introducing the regulation of protein function by phosphorylation, general structural properties of PIP-kinases will be described that are important for the understanding of the working hypotheses underlying experiments present in this thesis. 


\subsection{Structural properties of PIP-kinases}

Evidently, the activation of PIP-kinases is of great importance for the understanding of the PInetwork. In 1998, the structure of the human PIP-kinase II $\beta$ (HsPIPKII $\beta$ ) was crystalized (Rao et al., 1998) and the information gained from the structure aided in the understanding of how PIP-kinases associate with membranes and convert Ptdlns-monophophates to Ptdlnsbisphosphates. The three-dimensional model of the HsPIPKII $\beta$ (Fig. 1.5) illustrates the protein at a $3 \AA$ resolution and shows that the enzyme has a homodimeric structure.

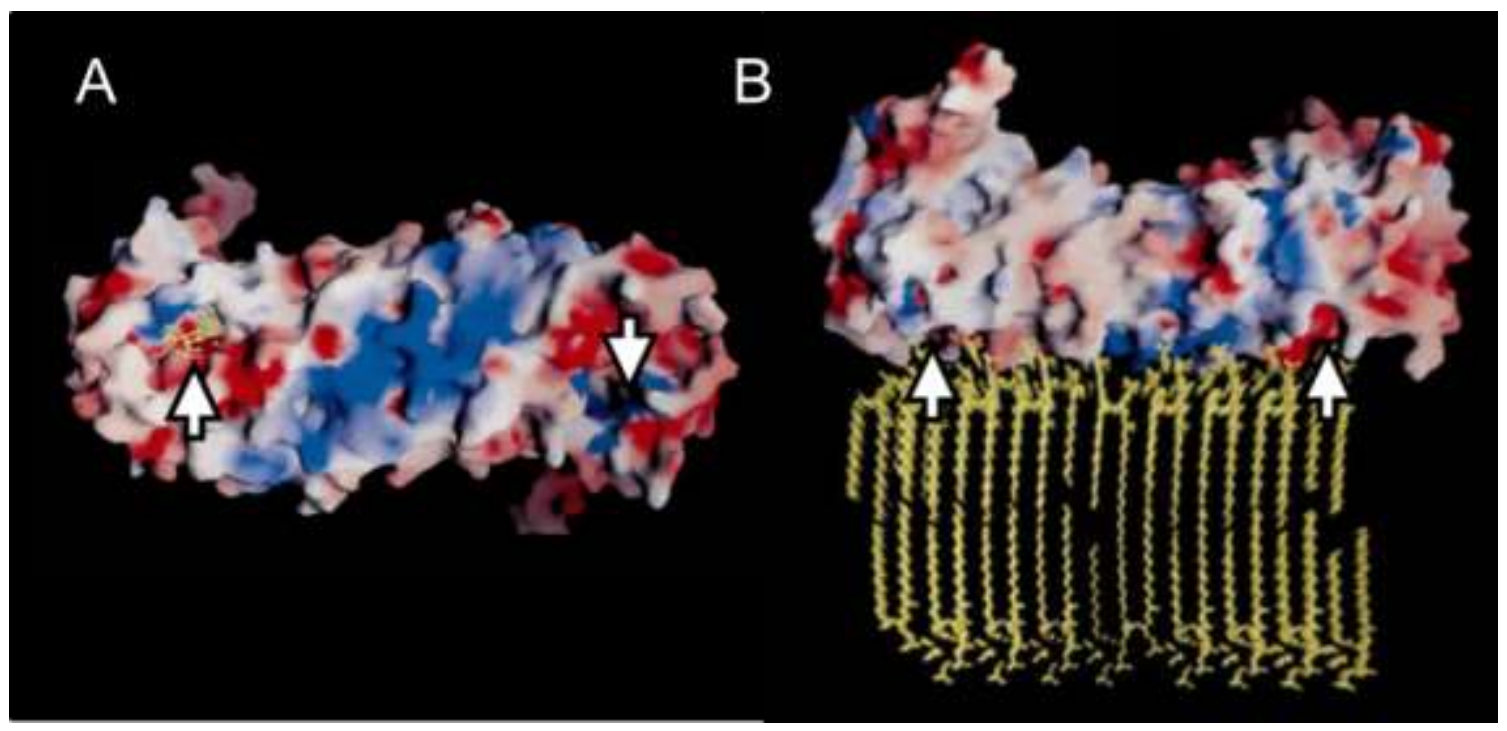

Fig. 1.5: Crystal structure of human PIPKIIß. The electrostatic potential is indicated by the colors and was calculated by GRASP (Graphical Representation And Analysis of Structural Proteins; (Honig and Nicholls, 1995). Red, Negative charges. Blue, Positive charges. A, Dimer in ventral orientation. ATP and PtdIns (yellow) are modulated into the catalytic centers (arrows). B, Association of HsPIPKII $\beta$ with the model membrane consisting of dimyristoyl-PtdCho (yellow). Figure modified from (Rao et al., 1998).

The HsPIPKII $\beta$ homodimer consists of two globular monomers that join their $\mathrm{N}$-terminal domains towards each other. The resulting homodimer presents a flattened surface towards the membrane (Rao et al., 1998). The homodimer is characterized by its unusually flat region at the ventral, membrane-oriented side of the protein that also contains many positivelycharged amino acid residues. The membrane interface region also holds the entrances to the catalytic site with the ATP binding pockets. It has been suggested that the flattened region facilitates membrane association by electrostatically interacting with negatively charged head groups of a membrane containing Ptdlns-monophosphates as substrate lipids (Rao et al., 1998). The structure of the HsPIPKII $\beta$ homodimer implies that the membrane association is based on weak interactions between enzyme and lipid bilayer (Burden et al., 1999). This concept is in contrast to observations on other membrane-associated signaling proteins, like protein kinase $C$ or phospholipase $A$, which penetrate the membrane and anchor the enzyme by hydrophobic interactions of specific domains (Hurley and Grobler, 1997). 
When basic amino acids of the HsPIPKII $\beta$ protein were mutated to acidic residues, the affinity of the enzyme towards substrate vesicles was severely decreased, suggesting that the electrostatic interaction between membrane and enzyme is necessary for catalytic function (Burden et al., 1999). In this proposed model for membrane association (Rao et al., 1998; Burden et al., 1999) the PI-substrate has access to the catalytic sites and is in optimal proximity to the ATP binding pocket. The reaction catalyzes the transfer of a phosphate group onto the Pl-substrate and subsequently the product is released (Rao et al., 1998). By associating with the membrane and phosphorylating its substrate lipid in situ, HsPIPKII $\beta$ bypasses the need to remove the substrate lipid partially or fully from the membrane and thus the reaction is energetically favored (Rao et al., 1998).

A possible means to regulate PIP-kinase activity might be based on the weak interactions of the PIP-kinase with the membrane, which are easily counteracted by phosphorylation, an important posttranslational modification which introduces negative charges (Rao et al., 1998; Burden et al., 1999). Based on the structure of the human PIP-kinase II $\beta$, phosphorylation especially at the positively-charged membrane-interface seems a likely means to regulate membrane association and activity, as it would interfere with the electrostatic interactions between enzyme and substrate membrane (Fig. 1.6). Thus, phosphorylation of PIP-kinases may influence catalytic activity by inhibiting the proper association of the enzyme with the substrate membrane. While this hypothesis suggests that PIP-kinases are removed from the membrane by phosphorylation at the membrane interface, it is also possible that phosphorylation prevents binding and conversion of the lipid substrate at the membrane even without complete dislocation of the enzyme from the membrane into the cytosol (Fig. 1.6).
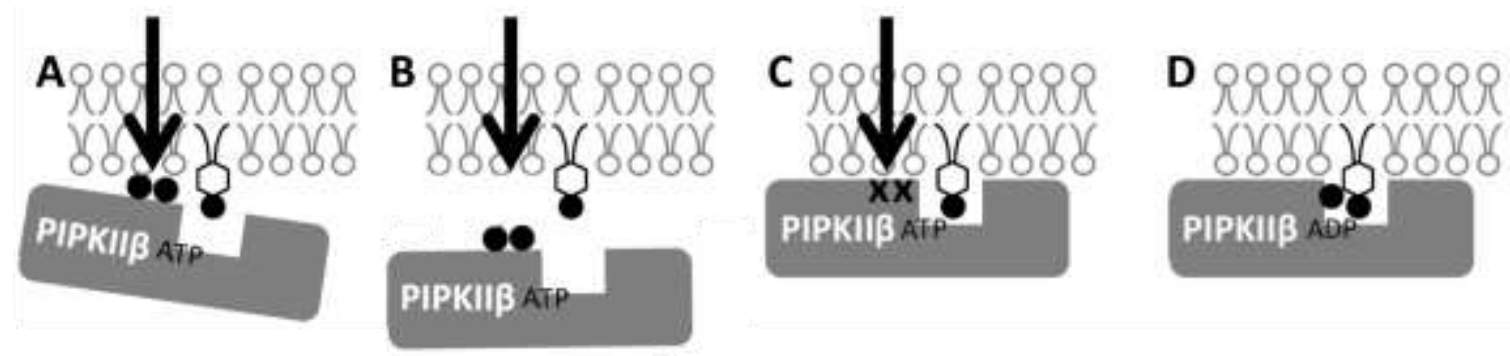

Fig. 1.6. A model of phosphorylation regulating PI4P 5-kinase activity and/or membrane association. $A$, Phosphorylation at the membrane interface (circles, arrow) interferes with the electrostatic interaction of the basic ventral side of human PIP-kinase II beta with anionic phospholipids. B, PIP5KII $\beta$ is removed from the membrane by phosphorylation at the membrane interface (circles arrow). C, Dephosphorylation (crosses, arrow) enables interaction with the substrate lipids. C, Proper membrane binding leads to the formation of $\operatorname{Ptd} \operatorname{lns}(4,5) \mathrm{P}_{2}$.

All structural data described have been obtained based on the study of the HsPIPKII $\beta$, an enzyme displaying marked differences to PIP-kinases from plants. HsPIPKII $\beta$ and the Arabidopsis PI4P 5-kinases that are the topic of this thesis share only between 25 and $36 \%$ 
sequence identity. Furthermore, HsPIPKII $\beta$ and the plant enzymes also differ in their preferred substrates and the regiospecificity of the catalysed reaction, HsPIPKII $\beta$ phosphorylating PtdIns5P in the D-4 position and the Arabidopsis enzymes phosphorylating Ptdlns4P in the D-5 position (Heilmann, 2009). In the absence of better structural data, it is a key working hypothesis of this thesis that PI4P 5-kinases from Arabidopsis display structural properties similar to those HSPIPKII $\beta$ and that a positively charged membrane interface region of Arabidopsis PI4P 5-kinases is the site of posttranslational modification by phosphorylation.

\subsection{Regulation of proteins by posttranslational modifications}

Posttranslational modifications extend the complexity of protein functions, since proteins can be modified by the addition of several chemical groups (Hunter, 2007; Yang and Seto, 2008). In this respect, protein phosphorylation is a widespread modification that has important regulatory effects on protein function. Reversible phosphorlyation is currently the best studied posttranslational modification (Reinders and Sickmann, 2005; Morandell et al., 2006) and functions in diverse cellular processes such as metabolism, transcription and translation, protein degradation, signal transduction, cell-to-cell communication, cell diversification and differentiation have been described (Graves and Haystead, 2003; Hunter, 2009).

Reversible phosphorylation of a protein can lead to temporary changes in its conformation, affecting catalytic activity, protein stability, interactions with other biomolecules or the subcellular localization of a protein (de la Fuente van Bentem et al., 2006). Protein phosphorylation in eukaryotic organisms mostly occurs on serines, threonines or tyrosines. Additionally, histidine and glutamate are also accessible for phosphorylation by specific protein kinases. Based on data from large scale experiments analyzing the phosphorylation status of Arabidopsis proteins it was shown that serine is the most detectable phosphorylation site ( 85 \%) followed by threonine (10.7 \%) and tyrosine (4.4. \%), respectively (Huang et al., 2003); (Sugiyama et al., 2008). A similar distribution was detected for the human phosphoproteinprofile (Olsen et al., 2006; Molina et al., 2007), suggesting that the basic regulatory machineries in eukaryotic organisms have been conserved in evolution. In plants protein phosphorylation can be triggered by light, phytohormones, lack of nutrients, pathogen invasion or changes in temperature (de la Fuente van Bentem et al., 2006; Kersten et al., 2009).

The complex interplay between phosphorylation and dephosphorylation occurs by the action of specific protein kinases and protein phosphatases that modify particular residues of their specific target proteins (Thingholm et al., 2009). Phosphorylation of a specific residue can then 
lead to conformational changes in the tertiary structure of the protein, exposing additional phosphorylation sites or sterically preventing the protein from further phosphorylation (Thingholm et al., 2009).

Since phosphorylation regulates diverse cellular processes, it is possible that rapid increases of PtdIns(4,5) $P_{2}$-levels observed upon application of external stimuli (Heilmann et al., 1999; Pical et al., 1999; DeWald et al., 2001; Heilmann et al., 2001; König et al., 2007; König et al., 2008) is due to activation/ inactivation of PI4P 5-kinases by phosphorylation or dephosphorylation. As will be described in the following section, reversible phosphorylation has indeed been described for PI4P 5-kinases from different eukaryotic model systems.

\subsection{Regulation of PI4P 5-kinases by phosphorylation}

There are several reports indicating protein phosphorylation as an important regulatory mechanism of PI4P 5-kinase-activitiy in animal, yeast and plant models. For Schizosaccharomyces pombe it was demonstrated that a PI4P 5-kinase can be phosphorylated by a casein kinase (CK1), decreasing the activity in vitro (Vancurova et al., 1999). For the PI4P 5-kinase Mss4p of Saccharomyces cerevisiae it has been shown that protein phosphorylation controls plasma membrane association (Audhya and Emr, 2003). In 2001 phosphorylation of the human Type I PIP-kinase (HsPIPK1) by protein kinase a (PKA) was reported to decrease catalytic activity (Park et al., 2001). Specific dephosphorylation of HsPIPK1 by a protein phosphatase reactivated the enzyme (Park et al., 2001). Similar observations were reported for plant enzymes. For instance it was shown that Arabidopsis PIP5K1 can also be phosphorylated in vitro by PKA (Westergren et al., 2001). Importantly, activity tests with phosphorylated PIP5K1 illustrated reduced specific activity in comparison to dephosphorylated protein (Westergren et al., 2001).

Despite of reports on in vitro phosphorylation of Arabidopsis PIP5K1, it remains unclear where in plant PI4P 5-kinases phosphorylation occurs, whether phosphorylation of plant PI4P 5kinases influences membrane association, and whether plant-endogenous protein kinases are involved with relevance for Ptdlns(4,5) $\mathrm{P}_{2}$ formation in vivo. Therefore, this thesis is concerned with regulation of two Arabidopsis PI4P 5-kinases by reversible phosphorylation. Arabidopsis PIP5K2 was chosen for this study, because it displays higher catalytic activity than PIP5K1 (Stenzel et al., 2008), to which it has high sequence similarity and because PIP5K2, like PIP5K1 is expressed in all organs of Arabidopsis (Stenzel et al., 2008). Arabidopsis PIP5K3 was chosen based on its even higher specific activity (Stenzel et al., 2008) and because PIP5K3 is 
specifically expressed in roots and seedlings (Stenzel et al., 2008), suggesting a potentially different mode of regulation than PIP5K2.

\subsection{Goals}

For various eukaryotic models it has been shown that PI4P 5-kinases are regulated by reversible phosphorylation, including PIP5K1 from Arabidopsis. In the context of this thesis two Arabidopsis PI4P 5-kinases, PIP5K1 and PIP5K3, were investigated for effects of phosphorylation on the in vitro and in vivo functionality and subcellular localization of the enzymes. The precise goals were as follows:

1. Characterize the in vitro phosphorylation of recombinant PI4P 5-kinases from Arabidopsis by PKA and by relevant plant extracts.

2. Determine possible phosphorylation sites by computer aided prediction and by Mass spectrometry (MS)-analysis on recombinant proteins.

3. Generate cDNA-clones of PIP5K2 and PIP5K3 by site directed mutagenesis to encode protein variants in which phosphorylation sites are altered to residues mimicking the dephosphorylated (A) or the phosphorylated state (D)

4. Express the variant enzymes in E. coli and test for effects on catalytic activity.

5. Express the variant enzymes as fusions to fluorescence tags in tobacco pollen tubes and test for effects on plasma membrane localization and pollen tube morphology.

6. Test variants of PIP5K2 and PIP5K3 for their capability to complement the phenotype of the available T-DNA insertion mutants. 


\section{Materials and Methods}

\subsection{Chemicals}

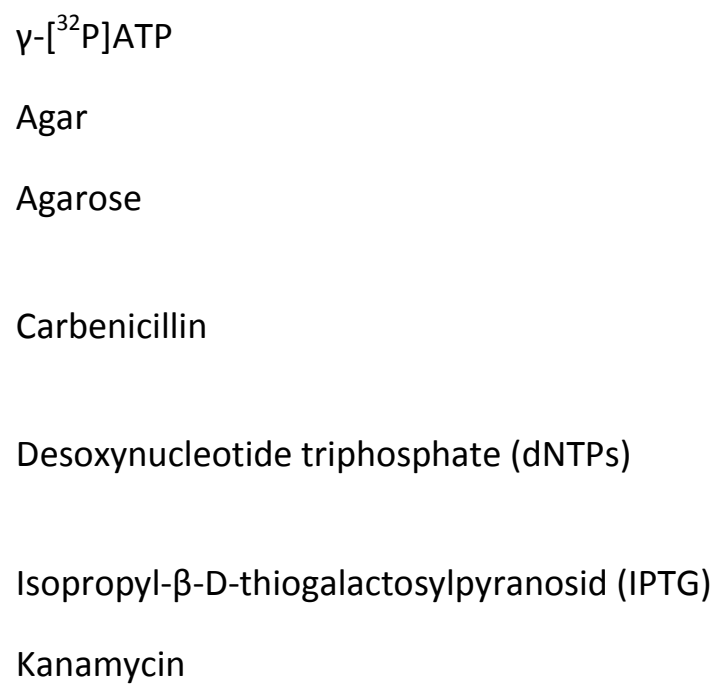

Hartmann Analytics, Braunschweig, Germany Invitrogen, Karlsruhe, Germany Duchefa Biochemie, Haarlem, The Netherlands

Duchefa Biochemie, Haarlem, The Netherlands

Roche Molecular Biochemicals, Mannheim, Germany

AppliChem, Darmstadt, Germany

Duchefa Biochemie, Haarlem, The Netherlands

Duchefa Biochemie, Haarlem, The Netherlands

Invitrogen, Karlsruhe, Germany

Roche Diagnostics GmbH, Mannheim, Germany

Avanti Polar Lipids Inc., Alabaster, AL, USA

Duchefa Biochemie, Haarlem, The Netherlands

OSi Specialties Inc., South Charleston, WI, USA

Bayer Crop Science, Mannheim, Germany

All other chemicals were purchased from the companies Roth (Karlsruhe, Germany), Merck (Darmstadt, Germany), Fluka (Steinheim, Germany) or Sigma-Aldrich (Deisenhofen, Germany). All media and solutions were prepared with filtered ultrapure water ( $\left(\mathrm{dd}_{2} \mathrm{O}\right)$. Media were sterilized for $20 \mathrm{~min}$ at $120^{\circ} \mathrm{C}$ in an autoclave (H+P Labortechnik, Oberschleißheim, Germany) before use. 


\subsection{Enzymes and size markers}

Gateway® LR Clonase ${ }^{\mathrm{TM}}$ Enzyme Mix

GeneRulerTM 1kb DNA-Ladder

Hot Start Taq-DNA Polymerase

Lysozyme

Pfu-PolymeraseTM

Phusion High Fidelity DNA-Polymerase

Protein kinase A (PKA) Catalytic subunit from bovine heart

Prestained Protein Molecular Weight Marker

Restriction endonucleases

Trypsin from bovine pancreas

Unstained Protein Molecular Weight Marker
Invitrogen, Karlsruhe, Germany

MBI Fermentas, St. Leon Rot, Germany

Peqlab, Erlangen, Germany

SERVA Electrophoresis $\mathrm{GmbH}$, Heidelberg Germany

MBI Fermentas, St. Leon Rot, Germany

NEB, Ipswich, MA, USA

Sigma-Aldrich, Deisenhofen, Germany

MBI Fermentas, St. Leon Rot, Germany

MBI Fermentas, St. Leon Rot, Germany

Sigma-Aldrich, Deisenhofen, Germany

MBI Fermentas, St. Leon Rot, Germany

\subsection{Kits}

Nucleospin Plasmid Kit

Nucleospin Extract II Kit

Plasmid Midi Core Kit

MBP Trap HP, 5 ml,
Macherey \& Nagel, Düren, Germany

Macherey \& Nagel, Düren, Germany

Qiagen, Hilden, Germany

GE Healthcare Life Sciences, Stockholm, Sweden

\subsection{Equipment}

ÄKTA FPLC

Fluorescence Stereo Microscope AXIO IMAGER M1

46HE Filterset for YFP

Helium driven particle delivery system PDS1000

LSM 510Meta
GE Healthcare Life Sciences, Stockholm, Sweden

Carl Zeiss, Jena, Germany

Carl Zeiss, Jena, Germany

Bio Rad, Munich, Germany

Carl Zeiss, Jena, Germany 
SDS gel rigs, SE 250

Tissue-Lyser MM200

FRENCH $^{\text {TM }}$-PRESS

RC10 and Jouan RCT 60

Fujifilm BAS-1500 phosphorimager
Hoefer scientific instruments, Holliston, MA, USA

Retsch, Haan, Germany

Gaulin, APV Homogeniser GmbH, Gatwick, UK

MedServ GmbH, Leipzig, Germany

Fujifilm, Düsseldorf, Germany

\subsection{Single-use materials}

Cellulose Acetate Filter $0.2 \mu \mathrm{m}$

Glass beads $2.85-3.3 \mathrm{~mm}$

Micron Gold Microcarriers, Stopping Screens, Rupture Disks (1350 psi)

PepStar $^{\mathrm{TM}}$ peptide microarrays

Silica 560 thin layer chromatography plates $20 \times 20 \mathrm{~cm}$

ZipTip U-C18, Tip Size P10
Sartorius, Göttingen, Germany

Roth, Karlsruhe, Germany

Bio Rad, Munich, Germany

JPT Peptide Technologies GmbH, Berlin, Germany

Merck, Darmstadt, Germany

Merck Millipore Corporation, Billerica, MA, USA

\subsection{Software}

Several software tools were used to process, image or sequence data as described in table 2.1.

Table 2.1: Software tools used in this thesis.

\begin{tabular}{|c|c|}
\hline ImageJ (http://rsbweb.nih.gov/ij/) & $\begin{array}{l}\text { Software for measuring root hair length } \\
\text { and pollen tube diameter }\end{array}$ \\
\hline Photoshop 7.0 (Adobe Systems, Munich, Germany) & Graphic tool for image processing \\
\hline $\begin{array}{l}\text { NetPhos } 2.0 \text { (CBS Prediction Center, Center For } \\
\text { Biological Sequence Analysis, TU Denmark. Freely } \\
\text { available at } \\
\text { http://www.cbs.dtu.dk/services/NetPhos)(Blom et } \\
\text { al., 1999) }\end{array}$ & $\begin{array}{l}\text { Identification of phosphorylation sites } \\
\text { in proteins by computer aided } \\
\text { prediction }\end{array}$ \\
\hline $\begin{array}{l}\text { PhosPhAt } 4.0 \text { www.cbs.dtu.dk/services/NetPhos/ } \\
\text { (Heazlewood et al., 2008) }\end{array}$ & $\begin{array}{l}\text { Database collecting experimentally } \\
\text { identified phosphorylation sites in } \\
\text { Arabidopsis proteins }\end{array}$ \\
\hline
\end{tabular}




\subsection{Plant lines}

Table 2.2: Plant lines used during this thesis.

\begin{tabular}{|c|c|c|c|}
\hline Name & Species & Transgenes & Obtained from \\
\hline Tobacco WT & $\begin{array}{l}\text { Nicotiana tabacum } \\
\text { Ecotype Samsun N }\end{array}$ & - & $\begin{array}{l}\text { Department of } \\
\text { Cellular Biochemistry } \\
\text { MLU Halle- } \\
\text { Wittenberg }\end{array}$ \\
\hline $\begin{array}{l}\text { Arabidopsis wild } \\
\text { type Col-0 }\end{array}$ & $\begin{array}{l}\text { Arabidopsis thaliana } \\
\text { Ecotype Columbia } 0\end{array}$ & - & $\begin{array}{l}\text { Department of } \\
\text { Cellular Biochemistry } \\
\text { MLU Halle- } \\
\text { Wittenberg }\end{array}$ \\
\hline $\begin{array}{l}\text { Arabidopsis pip5k1 } \\
\text { PIP5K1 pip5k2 } \\
\text { double mutant }\end{array}$ & $\begin{array}{l}\text { Arabidopsis thaliana } \\
\text { Ecotype Columbia } 0\end{array}$ & SALK cassette & $\begin{array}{l}\text { Department of } \\
\text { Cellular Biochemistry } \\
\text { MLU Halle- } \\
\text { Wittenberg }\end{array}$ \\
\hline $\begin{array}{l}\text { Arabidopsis pip5k1 } \\
\text { pip5k2 double } \\
\text { mutant } \\
\text { PIP5K2::PIP5K2 } \\
\text { T500A:EYFP }\end{array}$ & $\begin{array}{l}\text { Arabidopsis thaliana } \\
\text { Ecotype Columbia } 0\end{array}$ & $\begin{array}{l}\text { SALK cassette } \\
\text { PIP5K2::PIP5K2 } \\
\text { T500A:EYFP }\end{array}$ & $\begin{array}{l}\text { Generated during this } \\
\text { work }\end{array}$ \\
\hline $\begin{array}{l}\text { Arabidopsis pip5k1 } \\
\text { pip5k2 double } \\
\text { mutant } \\
\text { PIP5K2::PIP5K2 } \\
\text { T500D:EYFP }\end{array}$ & $\begin{array}{l}\text { Arabidopsis thaliana } \\
\text { Ecotype Columbia } 0\end{array}$ & $\begin{array}{l}\text { SALK cassette } \\
\text { PIP5K2::PIP5K2 } \\
\text { T500D:EYFP }\end{array}$ & $\begin{array}{l}\text { Generated during this } \\
\text { work }\end{array}$ \\
\hline $\begin{array}{l}\text { Arabidopsis pip } 5 k 3 \\
\text { mutant }\end{array}$ & $\begin{array}{l}\text { Arabidopsis thaliana } \\
\text { Ecotype Columbia } 0\end{array}$ & SALK cassette & $\begin{array}{l}\text { SALK institute, La } \\
\text { Jolla, CA, USA }\end{array}$ \\
\hline $\begin{array}{l}\text { Arabidopsis pip5k3 } \\
\text { mutant } \\
\text { PIP5K3::EYFP:PIP5K3 } \\
\text { T472A }\end{array}$ & $\begin{array}{l}\text { Arabidopsis thaliana } \\
\text { Ecotype Columbia } 0\end{array}$ & $\begin{array}{l}\text { SALK cassette } \\
\text { PIP5K3::PIP5K3 } \\
\text { T472A:EYFP }\end{array}$ & $\begin{array}{l}\text { Generated during this } \\
\text { work }\end{array}$ \\
\hline $\begin{array}{l}\text { Arabidopsis pip5k3 } \\
\text { mutant } \\
\text { PIP5K3::EYFP:PIP5K3 } \\
\text { T472D: }\end{array}$ & $\begin{array}{l}\text { Arabidopsis thaliana } \\
\text { Ecotype Columbia } 0\end{array}$ & $\begin{array}{l}\text { SALK cassette } \\
\text { PIP5K3::PIP5K3 } \\
\text { T472D:EYFP }\end{array}$ & $\begin{array}{l}\text { Generated during this } \\
\text { work }\end{array}$ \\
\hline
\end{tabular}




\subsection{Microorganisms}

\begin{tabular}{|c|c|c|c|}
\hline Organism & Strain & Genotype & Reference \\
\hline Escherichia coli & DH $5 \alpha$ & $\begin{array}{l}\text { fhuA2 } \Delta \text { (argF-lacZ)U169 phoA glnV44 } \\
\text { Ф80 } \Delta \text { (lacZ)M15 gyrA96 recA1 relA1 } \\
\text { endA1 thi-1 hsdR17 }\end{array}$ & - \\
\hline Escherichia coli & Rosetta 2 & 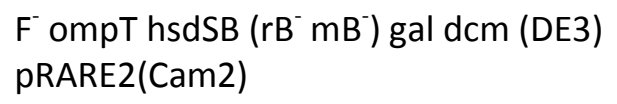 & - \\
\hline $\begin{array}{l}\text { Agrobacterium } \\
\text { tumefaciens }\end{array}$ & pEHA105 & pTiBo542 $\Delta$ T-DNA RifR & (Hood et al., 1993) \\
\hline
\end{tabular}

\subsection{Plasmids}

Table 2.4: Plasmids used in this thesis.

\begin{tabular}{|c|c|c|c|}
\hline Vector & $\begin{array}{l}\text { Selection } \\
\text { marker }\end{array}$ & Obtained from & Plasmid structure \\
\hline pETM41 & $A m p^{R}$ & $\begin{array}{l}\text { Dr. Achim Dickmanns, } \\
\text { Göttingen, Germany }\end{array}$ & $\begin{array}{l}\text { Plasmid contains an N-terminal MBP- } \\
\text { tag for purification and a TEV cleavage } \\
\text { site for removal of the MBP-tag }\end{array}$ \\
\hline pLatGW & $A m p^{R}$ & $\begin{array}{l}\text { Prof. Dr. Wolfgang } \\
\text { Dröge-Laser, } \\
\text { Würzburg, Germany }\end{array}$ & $\begin{array}{l}\text { Plasmid contains a Lat52 promotor } \\
\text { used for pollen-specific expression in } \\
\text { front of a Gateway cassette containing } \\
\text { attR1 and attR2 sequences required for } \\
\text { the Gateway }{ }^{\circledR} \text {-System }\end{array}$ \\
\hline $\begin{array}{l}\text { pCAMBIA } \\
3300-0 G C\end{array}$ & $A m p^{R}$ & $\begin{array}{l}\text { Dr. Ellen Hornung, } \\
\text { Göttingen, Germany }\end{array}$ & $\begin{array}{l}\text { Plasmid contains a BASTA resistence } \\
\text { under a } 35 \text { S promoter and a Gateway } \\
\text { cassette containing attR1 and attR2 } \\
\text { sequences required for the Gateway }{ }^{\circledR} \\
\text { System }\end{array}$ \\
\hline
\end{tabular}

\subsection{Protein expression and purification}

For the biochemical characterization of PIP5K2 and PIP5K3, the recombinant proteins were heterologously expressed in Escherichia coli (E. coli) as will be described in detail in the following sections. 


\subsubsection{Preparation of chemically-competent E. coli cells}

For transformation experiments chemically-competent $E$. coli cells were prepared as follows: Simple colonies were picked and precultured in $5 \mathrm{ml}$ of LB media (1\% (w/v) peptone, $0.5 \%$ $(\mathrm{w} / \mathrm{v})$ yeast extract, $1 \%(\mathrm{w} / \mathrm{v}) \mathrm{NaCl})$ over night at $37^{\circ} \mathrm{C}$ and continuous shaking at $120 \mathrm{rpm}$. $3 \mathrm{ml}$ of the starter culture were used to inoculate $250 \mathrm{ml}$ of LB media and cells were grown at $37{ }^{\circ} \mathrm{C}$ to an optical density $\left(\mathrm{OD}_{600}\right)$ of $0.6-0.8$. Cells were cooled down for $10 \mathrm{~min}$ and precipitated at $1000 \times \mathrm{g}$ for $10 \mathrm{~min}$ at $4{ }^{\circ} \mathrm{C}$. The media was removed and the pellet was washed in $80 \mathrm{ml}$ of cooled and sterilized TFB buffer (10 mM piperazine-N,N'-bis(2-ethanesulfonic acid) (PIPES), pH 6.7, $15 \mathrm{mM} \mathrm{CaCl}_{2}, 250 \mathrm{mM} \mathrm{KCl}, 55 \mathrm{mM} \mathrm{MnCl}_{2}$ ). After incubation on ice for another $10 \mathrm{~min}$, cells were centrifuged, resolved in $20 \mathrm{ml}$ of TFB buffer containing $7 \%(\mathrm{w} / \mathrm{v})$ dimethyl sulfoxide (DMSO) and the incubation step on ice was repeated. Finally, the cell suspension was collected in aliquots, immediately frozen in liquid nitrogen and stored at $-80 \mathrm{C}^{\circ}$ for further experiments.

\subsubsection{Transformation of chemically-competent $E$. coli cells}

Transformation of chemically-competent $E$. coli cells was achieved according to Inoue et al. (1990). $100 \mu \mathrm{l}$ of chemically-competent cells were thawed on ice and 1-10 $\mu \mathrm{l}$ of plasmid DNA were added. After incubation on ice for $30 \mathrm{~min}$, cells were heat-shocked at $42{ }^{\circ} \mathrm{C}$ for $40 \mathrm{~s}$ in a heating block and immediately transferred on ice for 2-5 min. $350 \mu \mathrm{l}$ of LB media were added and cells were grown at $37{ }^{\circ} \mathrm{C}$ for $1 \mathrm{~h}$. Finally, cells were plated on solid LB medium (as above solidified with $1.5 \%(\mathrm{w} / \mathrm{v})$ agar) containing the appropriate selection antibiotic (carbenicillin $100 \mu \mathrm{g} / \mathrm{ml}$, kanamycin $50 \mu \mathrm{g} / \mathrm{ml}$ ) and grown over night at $37^{\circ} \mathrm{C}$.

\subsubsection{Isolation of plasmid-DNA from bacterial cultures}

Plasmids were isolated from $2 \mathrm{ml}$ of liquid E. coli cultures using the NucleoSpin Plasmid Kit (Macherey-Nagel, Düren, Germany) according to the manufacturer's instructions. To generate larger amounts of DNA, plasmids where isolated from medium scale $(20 \mathrm{ml})$ E. coli cultures using the CompactPrep Plasmid Midi Core Kit (Qiagen, Hilden, Germany) following manufacturer's recommendations. For purification of DNA from solutions or agarose gel pieces the NucleoSpin Extract II Kit (Macherey-Nagel, Düren, Germany) was used following manufacturer's recommendations. 


\subsubsection{Recombinant expression of proteins in E. coli}

Recombinant proteins were expressed in E. coli Rosetta 2 cells, an E. coli strain possessing additional tRNAs for 7 codones rarely used in E. coli (AGA, AGG, AUA, CUA, GGA, CCC and CGG) and thus, enhancing the expression of eukaryotic proteins. E. coli Rosetta 2 cells were transformed with pETM41-PIP5K2 or pETM41-PIP5K3 vector constructs as described above. The expression vectors contained an N-terminal maltose binding protein (MBP)-tag for further purification by affinity chromatography and to enhance the solubility of the proteins. The constructs were kindly provided by Dr. Irene Stenzel (MLU Halle-Wittenberg, Germany). Transformed cells were picked from plate and cultured at $37{ }^{\circ} \mathrm{C}$ over night in $50 \mathrm{ml}$ of $2 \mathrm{YT}$ medium (1\% (w/v) tryptone, $0.5 \%(\mathrm{w} / \mathrm{v})$ yeast extract, $0.5 \%(\mathrm{w} / \mathrm{v}) \mathrm{NaCl}, 0.2 \%(\mathrm{w} / \mathrm{v})$ glucose) containing the appropriate antibiotics at $37{ }^{\circ} \mathrm{C}$ overnight. The preculture was used to inoculate a baffled flask with $300 \mathrm{ml}$ of $2 \mathrm{YT}$ to an $\mathrm{OD}_{600}$ of 0.1 . Cells were grown at $37^{\circ} \mathrm{C}$ and protein expression was induced with $1 \mathrm{mM}$ isopropyl-1-thio- $\beta$-D-galactopyranoside (IPTG) at an $\mathrm{OD}_{600}$ of 0.7 - 0.8. After induction the culture was shaken at $130 \mathrm{rpm}$ for $4 \mathrm{~h}$ and $28^{\circ} \mathrm{C}$. Cells were subsequently harvested by centrifugation for $20 \mathrm{~min}$ at $10000 \times \mathrm{g}$. The bacterial pellet was resuspended in $40 \mathrm{ml}$ of binding buffer ( $50 \mathrm{mM}$ Tris $\mathrm{HCl}, \mathrm{pH}$ 7.5, $300 \mathrm{mM} \mathrm{NaCl}, 1 \mathrm{mM}$ EDTA) containing $0.1 \mathrm{mM}$ phenylmethanesulfonylfluoride (PMSF) and $1 \mathrm{mg} / \mathrm{ml}$ lysozyme (Serva Electrophoresis, Heidelberg, Germany) was added to initiate cell wall break down. After incubation on ice for $1 \mathrm{~h}$, the cellular disintegration was further aided by disrupting the cells in a FRENCH ${ }^{\text {TM }}$-PRESS (Gaulin, APV Homogeniser GmbH, Gatwick, UK) at 1200 bar. Membrane particles were removed by centrifugation for $20 \mathrm{~min}$ at $20000 \mathrm{xg}$ and $4{ }^{\circ} \mathrm{C}$ and the supernatant was kept cold for further purification steps.

\subsubsection{Purification of proteins by affinity chromatography}

Affinity chromatography is a widely used method to separate complex protein mixtures. The method is based on the high affinity of specific protein domains used as affinity-tags towards specific chemical groups such as sugars or metals. These groups are immobilized on a matrix and packed onto a column (immobile phase). By applying the protein mixture to the immobile phase, the target protein will bind to the matrix, whereas other proteins with lower binding affinity will flow through. After washing the immobile phase, the target protein can be eluted by applying a solution that a) has a higher affinity towards the matrix and thus competes with the target protein for binding capacity or b) contains a high concentration of the chemical group that is bound by the target protein and thus leads to elution of the protein from the immobile phase. 
In this thesis, recombinantly expressed proteins were fused to an N-terminal MBP-tag. The MBP-tag has a high affinity towards maltose and thus binds to columns packed with Dextrin Sepharose $\mathrm{T}^{\mathrm{TM}}$. Bound MBP can be eluted by applying a solution containing high concentrations of maltose. The purification procedure was simplified by using an ÄKTA ${ }^{T M}$-protein purification system (ÄKTA FPLC, GE Healthcare Life Sciences, Stockholm, Sweden). Crude cell lysates were applied with a flow rate of $1 \mathrm{ml} / \mathrm{min}$ onto a $5 \mathrm{ml} \mathrm{MBP}$ Trap (GE Healthcare Life Sciences, Stockholm, Sweden) equilibrated with binding buffer. The column was washed with binding buffer until the base line was reached again and the wash through was collected to document purification. Bound protein was eluted by applying elution buffer $(10 \mathrm{mM}$ maltose in binding buffer) with a flow of $1 \mathrm{ml} / \mathrm{min}$ and collected in fractions of $1 \mathrm{ml}$.

\subsubsection{Estimation of protein contents}

Protein concentrations were determined according to (Bradford, 1976) by mixing $5 \mu l$ of the sample with $995 \mu \mathrm{l}$ of Bradford reagent (0.01\% (w/v) Coomassie blue R 250, 5 \% (v/v) ethanol, $8,5 \%(\mathrm{v} / \mathrm{v})$ o-phosphoric acid) and measuring the absorption at $595 \mathrm{~nm}$. Protein concentrations were calibrated with BSA. Successful purification was verified by immunoblot analysis and in vitro activity tests.

\subsubsection{Separation of proteins via sodium dodecyl sulfate poly acrylamide gel electrophoresis (SDS-PAGE)}

Proteins were separated on $10 \%$ SDS gels according to (Laemmli, 1970). Stacking gels were composed of $0.66 \mathrm{ml}$ acrylamide/ bis-acrylamide ( $30 \% / 0.8 \%(\mathrm{w} / \mathrm{v})$ ), $0.8 \mathrm{ml} 0.6 \mathrm{M} \mathrm{Tris} \mathrm{HCl}, \mathrm{pH}$ 6.8, $2.5 \mathrm{ml} \mathrm{H}_{2} \mathrm{O}, 40 \mu \mathrm{l} 10 \%(\mathrm{w} / \mathrm{v})$ SDS and $2.5 \mu \mathrm{l}$ tetramethylethylenediamine (TEMED). Running gels consisted of $2 \mathrm{ml}$ acrylamide/ bis-acrylamide (30\%/ $0.8 \%(\mathrm{w} / \mathrm{v})$ )), $2.2 \mathrm{ml} 1.8 \mathrm{M}$ Tris $\mathrm{HCl}, \mathrm{pH} 8.8,2.75 \mathrm{ml} \mathrm{H} \mathrm{O}_{2}, 60 \mu \mathrm{l} 10 \%$ (w/v) SDS and $6 \mu$ I TEMED. The gels were polymerized by adding $0.5 \%(\mathrm{w} / \mathrm{v})$ ammonium persulfate (APS) and casted in a multiple gel caster (SE 200 series, Hoefer scientific instruments, Holliston, MA, USA). Before loading, protein samples were denaturated by adding 1/5 SDS sample buffer (225 mM Tris $\mathrm{HCl}, \mathrm{pH} 6.8,5 \%$ (w/v) glycerol, 5 \% (w/v) SDS, $0.05 \%$ (w/v) Coomassie blue R 250, $250 \mathrm{mM}$ dithiothreitol (DTT)) and heating at $95{ }^{\circ} \mathrm{C}$ for $5 \mathrm{~min}$. Gels were run at $25 \mathrm{~mA} /$ gel for $1 \mathrm{~h}$ in gel tanks (SE 250, Hoefer scientific instruments, Holliston, MA, USA) containing SDS running buffer ( 25 mM Tris, 200 mM glycine, $0.3 \mathrm{mM}$ SDS). The gels were then either subjected to immunodetection (see section 
2.7) or stained with Coomassie staining solution (40\% (v/v) methanol, $10 \%(\mathrm{v} / \mathrm{v})$ acetic acid und 0,25 \% (w/v) Coomassie Brilliant BlueG250).

\subsubsection{Immunodetection of specific proteins}

Recombinant proteins were detected by a western blot analysis using specific antibodies. Denaturated proteins were first separated by SDS-PAGE, and then transferred onto a nitrocellulose membrane (Optitran BA-S 85, $0.45 \mu \mathrm{m}$, Whatman, Maidstone, UK) via a wet blot cell (Biorad, Munich, Germany). The transfer was carried out in blotting buffer $(0.6 \%(\mathrm{w} / \mathrm{v})$ Tris, 0,3\% (w/v) glycine, $0.4 \%(\mathrm{w} / \mathrm{v})$ SDS, $20 \%$ methanol (v/v)) at $60 \mathrm{~V}$ for $1 \mathrm{~h}$. After blotting the nitrocellulose membrane was blocked with $5 \%$ (w/v) BSA in TBS buffer $(20 \mathrm{mM}$ Tris $\mathrm{HCl}$, $\mathrm{pH}$ 7.5, $50 \mathrm{mM} \mathrm{NaCl}$ ) overnight and washed in TBS buffer for $5 \mathrm{~min}$. The membrane was incubated with a primary a MBP-antibody (1:10000 in TBS buffer, Sigma) for $3 \mathrm{~h}$ at $4{ }^{\circ} \mathrm{C}$, following 2 washing steps with TBS buffer for 10 min each and an incubation for $1 \mathrm{~h}$ with a secondary antibody. The secondary antibody was conjugated to an alkaline phosphatase (AP) that catalyzes the reaction of nitro blue tetrazolium chloride (NBT) and 5-bromo-4-chloro-3indolyl phosphate (X-Phosphate) into a blue colourant. After incubation with the second antibody the washing steps with TBS buffer were repeated and the membrane was equilibrated in AP buffer (100 mM Tris $\mathrm{HCl}, \mathrm{pH} 9.5,100 \mathrm{mM} \mathrm{NaCl}, 5 \mathrm{mM} \mathrm{MgCl} 2)$ for 10 min. For the development $10 \mathrm{ml}$ of fresh AP buffer were added containing $35 \mu \mathrm{X}$-phosphate $(50 \mathrm{mg} / \mathrm{ml}$ in $100 \%$ Dimethylformamid (DMF)) and $45 \mu \mathrm{l} \mathrm{NBT} \mathrm{(75} \mathrm{mg/ml} \mathrm{in} 70 \%$ DMF). The staining procedure was terminated by washing the membrane in $\mathrm{dd}^{\mathrm{H}} \mathrm{H}_{2} \mathrm{O}$.

\subsubsection{In vitro activity test of recombinant PI4P 5-kinases with $\mathrm{Y}-\left[{ }^{32} \mathrm{P}\right]$ ATP}

The activity of recombinantly expressed PI4P 5-kinases was tested according to (Cho and Boss, 1995) by measuring the incorporation of radiolabeled $y-\left[{ }^{32} P\right]$ ATP into defined lipid substrates. For each reaction $6.25 \mu \mathrm{l}$ of a $1 \mathrm{mg} / \mathrm{ml}$ Ptdlns4P solution (Avanti Polar Lipids Inc., Alabaster, AL, USA) were dried under a stream of nitrogen and $5 \mu \mathrm{l} 2 \%(\mathrm{v} / \mathrm{v})$ triton X-100 were added. The dried lipids were evenly resuspended in a water bath sonificator for $10 \mathrm{~min}$. To assay PI4P 5kinase activity 1-1.5 $\mu \mathrm{g}$ of recombinantly expressed protein was mixed with $15 \mathrm{mM} \mathrm{MgCl}$, $1 \mathrm{mM} \mathrm{Na}_{2} \mathrm{MoO}_{4}, 1 \mathrm{mM}$ ATP, $10 \mu \mathrm{Ci} \psi-\left[{ }^{32} \mathrm{P}\right]$ ATP, $1 \mathrm{mM}$ Tris $\mathrm{HCl}, \mathrm{pH} 7.2$ and Ptdlns4P in a total volume of $50 \mu \mathrm{l}$. After an incubation at room temperature for $1 \mathrm{~h}$ the lipid products were extracted with $1.5 \mathrm{ml}$ chloroform:methanol (1:2 (v/v)), $250 \mu \mathrm{l} 0.5 \mathrm{mM}$ EDTA, $500 \mu \mathrm{l} 2.4 \mathrm{M} \mathrm{HCl}$ and $500 \mu \mathrm{l}$ chloroform according to (Cho et al., 1992). The samples were mixed, the organic 
phase was transferred into a new glass reaction tube and the aqueous phase was reextracted with $500 \mu$ l chloroform. The organic phases were combined and washed with $1 \mathrm{ml} 0.5 \mathrm{M} \mathrm{NaOH}$ in methanol:water $(1: 1(\mathrm{v} / \mathrm{v}))$. After removal of the aqueous phase, the extracted lipids were dried under a stream of nitrogen and dissolved in $20 \mu \mathrm{l}$ chloroform:methanol $(2: 1(\mathrm{v} / \mathrm{v}))$. The lipids were then separated via thin layer chromatography by applying the lipid products onto silica S60 plates (Merck, Darmstadt, Germany) and chloroform:methanol:ammonium hydroxide:water (45:45:4:11 (v/v/v/v)) as a developing solvent. Lipids were identified by comigration of authentic standards (5 $\mu$ g Ptdlns $4 P, 5 \mu \mathrm{g}$ Ptdlns $(4,5) \mathrm{P}_{2}$, Avanti polar lipids Inc., Alabaster, AL, USA) on the same plate. Lanes with lipids standards were cut and visualized by dipping the plate into an aqueous solution of $10 \%(\mathrm{w} / \mathrm{w}) \mathrm{CuSO}_{4}$ and $8 \%(\mathrm{w} / \mathrm{v})$ phosphoric acid followed by heating to $180{ }^{\circ} \mathrm{C}$ (König et al, 2007). Radiolabled lipids were visualized by exposing an imager screen (BAS-MP 2040 s, Fujifilm, Düsseldorf, Germany) and the extent of ${ }^{32} \mathrm{P}$-incorporation was quantified by a phosphorimager (Fujifilm BAS-1500, Fujifilm, Düsseldorf, Germany).

\subsection{Detection of phosphorylation sites of in vitro-phosphorylated PIP5K2 and PIP5K3}

Phosphoproteomics is a branch of proteomics that identifies phosphorylated proteins and maps detected phosphorylation sites (Kersten et al., 2009). In some cases it is also possible to assign corresponding protein kinases and phosphatases (Kersten et al., 2009). Little is known about the plant phosphoproteome, making the identification of possible phosphorylation sites difficult. Furthermore, the identification of putative phosphorylation sites based on known recognition sites, as has been shown for human protein kinases, is not possible to date for plant protein kinases (Kersten et al., 2009). Therefore, alternative approaches were needed to investigate the phosphorylation status of PIP5K2 and PIP5K3 from Arabidopsis as will be described in the next section.

\subsubsection{In vitro phosphorylation assays}

The phosphorylation status of PIP5K2 and PIP5K3 was determined by phosphorylating the enzymes with a commercially available PKA or with Arabidopsis protein kinases. Arabidopsis subcellular fractions were used as source for endogenous protein kinase activity. 


\section{Arabidopsis thaliana plant growth and subcellular fractionation}

Arabidopsis plants destined for the preparation of extracts, were sown on soil (Type Substrate 1, Klasmann-Deilmann $\mathrm{GmbH}$, Geeste, Germany), vernalized over night at $4{ }^{\circ} \mathrm{C}$ and grown under short day conditions with $8 \mathrm{~h}$ of light (7000 lux) and $16 \mathrm{~h}$ of darkness at $20-22{ }^{\circ} \mathrm{C}$ in climate growth-chambers (A 1000, Adaptis, Conviron, Winnipeg, Canada). Leaves of 6-weeksold plants were harvested, snap frozen in liquid nitrogen and stored at $-80{ }^{\circ} \mathrm{C}$. For the preparation of plant extracts from seedlings, Arabidopsis plants were cultivated under sterile conditions. Seeds were surface-sterilized with $6 \%(\mathrm{v} / \mathrm{v})$ sodium hypochlorite and $0.3 \%(\mathrm{v} / \mathrm{v})$ triton $\mathrm{X}-100$ for $12 \mathrm{~min}$ and washed with sterile $\mathrm{H}_{2} \mathrm{O}$ five times. Seeds were then plated on sterile Petri-dishes containing MS growth medium $(0.22 \%$ (w/v) Murashige \& Skoog medium, $1.5 \%(\mathrm{w} / \mathrm{v})$ sucrose, $1 \%(\mathrm{w} / \mathrm{v})$ agar) and grown under the conditions described for soil cultures. After 2 weeks of growth plants were harvested, immediately snap frozen in liquid nitrogen and stored at $-80^{\circ} \mathrm{C}$. For extracts prepared from plants challenged with salt stress, Arabidopsis seeds were surface-sterilized as described above and sown on MS growth medium that was cast sloped in rectangular Petri-dishes (see Fig. 2.1). Seeds were plated on the upper site of the MS growth medium and grown vertically under the conditions described for soil cultures. After 2 weeks of growth Petri-dishes were placed horizontally for $1 \mathrm{~h}$ in order to equilibrate plants to gravitropic stimuli. Afterwards roots were overlayed with liquid MS medium (without agar) containing $0.4 \mathrm{M} \mathrm{NaCl}$, the sloped $\mathrm{MS}$ growth medium prevented the leaves from exposure to the salt medium. Leaves were harvested prior to stress application and after 15, 30 and 60 min of salt stress, immediately snap frozen in liquid nitrogen and stored at $-80^{\circ} \mathrm{C}$.

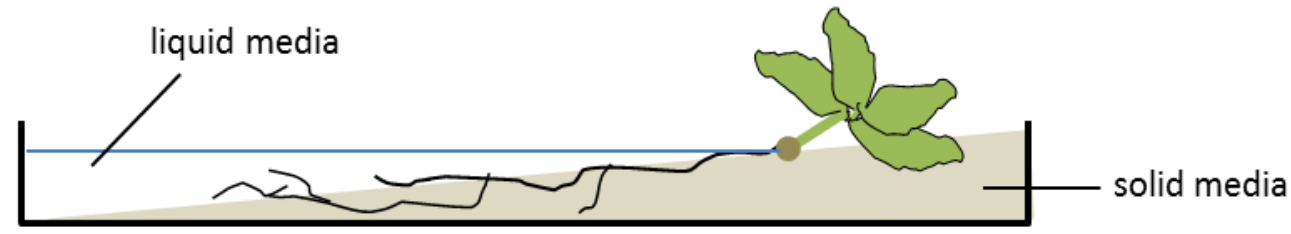

Fig. 2.1: Stress treatment of Arabidopsis plants sown on sloped MS growth medium. For salt-stress treatment Arabidopsis seeds were sown on MS medium that was cast sloped on Petri-dishes (grey). Plants were grown vertically for 2 weeks, placed horizontally and roots were exposed to liquid MS medium containing $0.4 \mathrm{M} \mathrm{NaCl}$ (blue line). Plant leaves (green) were not touched by the salt medium.

In cells, protein kinases are only active when phosphorylated themselves and the maintenance of their phosphorylation status is important. Therefore it was necessary to add appropriate phosphatase inhibitors to extraction buffers directly before use (Dissmeyer and Schnittger, 2011). Furthermore, protease inhibitors were included to prevent protein degradation. The inhibitors used are listed in Table 2.5. 
Table 2.5: List of inhibitors used for the preparation of Arabidopsis leaf extract.

\begin{tabular}{lll}
\hline Inhibitor & Inhibition of & Final concentration \\
\hline $\begin{array}{l}\text { PhosSTOP (Roche Diagnostics } \\
\text { GmBH, Mannheim, Germany) }\end{array}$ & $\begin{array}{l}\text { acid and alkaline phosphatases, } \\
\text { serine/threonine (PP1, PP2A, and } \\
\text { PP2B) and tyrosine protein } \\
\text { phosphatases (PTP) }\end{array}$ & $\begin{array}{l}\text { According to } \\
\text { manufacturer's } \\
\text { instructions }\end{array}$ \\
$\mathrm{Na}_{3} \mathrm{VO}_{4}$ & $\begin{array}{l}\text { Tyrosin phosphatases } \\
\mathrm{NaF}\end{array}$ & $1 \mathrm{mM}$ \\
$\begin{array}{l}\text { Protease inhibitor cocktail } \\
\text { (Sigma Aldrich, Germany) }\end{array}$ & $\begin{array}{l}\text { serine, cysteine, aspartic, and } \\
\text { metalloproteases, aminopeptidases }\end{array}$ & $\begin{array}{l}\text { According to } \\
\text { manufacturer's } \\
\text { instructions }\end{array}$ \\
\hline
\end{tabular}

Protein extracts were prepared from Arabidopsis-tissue by grinding deep-frozen material with mortar and pestle and immediately homogenizing the tissue powder on ice in 2 volumes of cold leaf extract - buffer (100 mM Tris, pH 7.5, $100 \mathrm{mM} \mathrm{MgCl}_{2}, 0.5 \mathrm{M} \mathrm{NaCl}, 1 \mathrm{mM}$ ethylene glycol tetraacetic acid (EGTA), $1 \mathrm{mM}$ DTT) including the appropriate inhibitors described above. Homogenates were centrifuged at $14000 \times \mathrm{g}$ for $15 \mathrm{~min}$ in a precooled centrifuge and the protein concentration of the supernatant was determined using Bradford. It was important to prepare protein extracts always freshly and to keep them on ice during all experiments to prevent loss of protein kinase activity or protein degradation.

\section{In vitro phosphorylation assays with radiolabeled $\mathrm{p}-\left[{ }^{32} \mathrm{P}\right]$ ATP}

The basic principle of in vitro phosphorylation assays is the use of radiolabeled $y-\left[{ }^{32} \mathrm{P}\right]$ ATP as a phosphate donor. In case of phosphorylation, the radiolabeled terminal $\gamma-\left[{ }^{32} \mathrm{P}\right]$-phosphate of the ATP molecule is transferred onto the protein of interest which can then be visualized by running an SDS-PAGE and exposing a radiosensitive film. Since radiolabeled $\mathrm{\gamma}-\left[{ }^{32} \mathrm{P}\right]$ ATP is only applied in nanomolar concentrations it is necessary to include cold ATP in the reaction mix in order to reach the $\mathrm{K}_{\mathrm{M}}$-value of the protein kinases used. If the cold ATP concentration is too low, phosphorylation of PIP5K2/PIP5K3 will consume a longer time range and thus increasing the possibility of protein kinase degradation or inactivation. If reciprocally the concentration of cold ATP is too high, less protein will be phosphorylated with radiolabeled ${ }^{32} \mathrm{P}$, thus, decreasing the detectability. The optimal cold ATP concentration was titrated when establishing the assay. For phosphorylation assays 5-10 $\mu \mathrm{g}$ of purified PI4P 5-kinase were phosphorylated with 4 units of PKA or $30 \mu \mathrm{g}$ of freshly prepared Arabidopsis plant extract. PKA, leaf extract, denaturated leaf extract and PI4P 5-kinase alone were used as negative controls. Leaf extract was 
denaturated by boiling at $95{ }^{\circ} \mathrm{C}$ for $20 \mathrm{~min}$. The samples were prepared in a $50 \mu \mathrm{l}$ reaction containing $100 \mathrm{mM}$ Tris, $\mathrm{pH}$ 7.5, $100 \mathrm{mM} \mathrm{MgCl}, 0.5 \mathrm{M} \mathrm{NaCl}, 1 \mathrm{mM}$ EGTA, $1 \mathrm{mM}$ DTT, phosphatase inhibitor cocktail (PhosSTOP, Roche Diagnostics GmBH, Mannheim, Germany) 50 $\mu \mathrm{M}$ cold ATP and $10 \mu \mathrm{Ci} \gamma-\left[{ }^{32} \mathrm{P}\right]$ ATP (Hartmann Analytics, Braunschweig, Germany). The samples were mixed and incubated at room temperature for $1 \mathrm{~h}$. If part of the experimental setup $1 \mu \mathrm{l}$ tobacco etch virus (TEV) -protease $(1 \mathrm{mg} / \mathrm{ml}$, kindly provided from Dr. Annett Weidner, MLU Halle) or shrimp alkaline phosphatase (SAP) (MBI Fermentas, St. Leon Rot, Germany) was added afterwards and additionally incubated for $1 \mathrm{~h}$ at room temperature. The reaction was stopped by adding $10 \mu \mathrm{l}$ of SDS sample buffer and heating the samples at $95{ }^{\circ} \mathrm{C}$ for $5 \mathrm{~min}$. Afterwards the denaturated proteins were separated on $10 \%$ SDS gels, the gels were stained with Coomassie staining solution and dried by clamping the gels over night between two cellophane films soaked with $3 \%(\mathrm{v} / \mathrm{v})$ glycerol, $30 \%(\mathrm{v} / \mathrm{v})$ methanol and $10 \%$ ( $\mathrm{v} / \mathrm{v}$ ) acetic acid. Radiolabeled protein bands were visualized by exposing a radiosensitive film (BAS-MP 2040 s, Fujifilm, Düsseldorf, Germany). The extent of ${ }^{32} \mathrm{P}$-incorporation was quantified by a phosphorimager (Fujifilm BAS-1500, Fujifilm, Düsseldorf, Germany).

\subsubsection{Tryptic in gel digestion of phosphorylated recombinant PI4P 5-kinases}

To detect phosphorylation sites with MS-analysis, 20-30 $\mu \mathrm{g}$ purified PI4P 5-kinase was first phosphorylated with plant extract from 6-weeks-old Arabidopsis plants as described above with the exception that the reaction mix included $5 \mathrm{mM}$ ATP and no $\mathrm{v}-\left[{ }^{32} \mathrm{P}\right]$ ATP was used. When a complex sample mixture is analyzed using MS-analysis, it is possible that highly abundant proteins mask the detection of peptides with low abundance. Furthermore, a mass spectrum of a highly complex sample results in an overwhelming number of different components and thus is difficult to analyze. That is why proteins were separated first via SDSPAGE, stained with Coomassie staining solution prior to MS-analysis and only the protein bands corresponding to PIP5K2 or PIP5K3 were used for further analysis. In addition, it was important to minimize contamination, especially with keratins, during sample preparation.

In preparation for MS-analysis the protein bands corresponding to PIP5K2 and PIP5K3 were extracted and the gel pieces were digested with trypsin according to (Shevchenko et al., 1996) since peptides ionize and fragment easier and more efficiently than proteins. In brief, gel slices extracted from SDS-PAGE-gels were macerated and destained by adding $100 \mu \mathrm{l}$ of $\mathrm{dd} \mathrm{H}_{2} \mathrm{O}$. After agitation for $10 \mathrm{~min}$ at room temperature the supernatant was discarded and the washing step was repeated. Then $100 \mu \mathrm{l}$ of destaining solution (30\% (v/v) acetonitrile in $100 \mathrm{mM} \mathrm{NH}_{4} \mathrm{HCO}_{3}, \mathrm{pH}$ 8.5) were added, the samples were incubated at room temperature for 
15 min with agitation and the supernatant was discarded. This procedure was repeated 4 times. After a second cycle of washing, incubation and decanting with $100 \mu \mathrm{ldd} \mathrm{H}_{2} \mathrm{O}$ and $100 \mu \mathrm{l}$ acetonitrile respectively, the gel pieces were dried by a centrifugal evaporator linked to a refrigerated cold trap (RC10 and Jouan RCT 60, MedServ GmbH, Leipzig, Germany). The dried gel slices were reduced with $40 \mu \mathrm{l}$ of $10 \mathrm{mM}$ DTT in $100 \mathrm{mM} \mathrm{NH}_{4} \mathrm{HCO}_{3}, \mathrm{pH} 8.5$, incubated for 5 min at room temperature and $30 \mathrm{~min}$ at $50{ }^{\circ} \mathrm{C}$. The supernatant was discarded, the samples were washed with $100 \mu \mathrm{l}$ acetonitrile and alkylated by adding $40 \mu \mathrm{l}$ of $54 \mathrm{mM}$ iodoacetamide in $100 \mathrm{mM} \mathrm{NH}_{4} \mathrm{HCO}_{3}, \mathrm{pH}$ 8.5. After incubation for $15 \mathrm{~min}$ at room temperature in the dark, the supernatant was discarded, the samples were washed twice with $100 \mu \mathrm{l}$ of destaining solution and the gel slices were dried. For digestion the dried gel slices were covered with trypsin solution ( $3 \mathrm{ng} / \mu$ l trypsin (Sigma-Aldrich, Deisenhofen, Germany), $50 \mathrm{mM} \mathrm{NH}_{4} \mathrm{HCO}_{3}, \mathrm{pH} 8.5,5 \%$ ( $\mathrm{v} / \mathrm{v}$ ) acetonitrile) and incubated over night at room temperature. Trypsin cleaves peptides in the C-terminal position next to arginine and lysine residues. Peptides were extracted with a solution of $50 \%(\mathrm{v} / \mathrm{v})$ acetonitrile and $0.1 \%(\mathrm{v} / \mathrm{v})$ trifluoroacetic acid (TFA) (same volume as trypsin solution) for $40 \mathrm{~min}$ with agitation and the supernatant was transferred into a new reaction tube. The extraction was repeated with incubation for $15 \mathrm{~min}$ and the supernatants were combined. The peptides were dried, sonicated in $20 \mu \mathrm{l}$ of $0.1 \%(\mathrm{v} / \mathrm{v})$ TFA for $5 \mathrm{~min}$ and stored at $-20^{\circ} \mathrm{C}$ for further analysis.

\section{Desalting of peptide solutions}

MS-analysis is based on the ionization of molecules. Therefore it is important to minimize the abundance of other ions such as sodium and phosphate salts that can increase the detection background. Peptide solutions were 32desalted and concentrated by using ZipTips (Merck Millipore Corporation, Billerica, MA, USA) filled with $\mathrm{C}_{18}$ silicia resin. ZipTips were washed with $75 \%(\mathrm{v} / \mathrm{v})$ acetonitrile twice and equilibrated with $0.1 \%(\mathrm{v} / \mathrm{v})$ TFA in $\mathrm{dd} \mathrm{H}_{2} \mathrm{O}$. For sample binding ZipTips were aspirated and dispensed through the peptide solution media 10 times. After washing the tip with $0.1 \%(\mathrm{v} / \mathrm{v})$ TFA for 3 times, peptides were eluted with $0.1 \%(\mathrm{v} / \mathrm{v})$ TFA in $75 \%(v / v)$ acetonitrile.

\section{MS-analysis of tryptic peptide mixtures}

MS is an analytical method to determine molecule masses. It depends on the ionization of chemical compounds or molecule fragments that are then separated based on their mass-tocharge-ratio ( $\mathrm{m} / \mathrm{z}$ ) as will be described briefly in the following section (Vollhardt, 2005). 
After loading the sample of interest onto the mass spectrometer it enters the gas phase and the molecules are transformed into charged ions by emitting electrons. There are different methods to ionize a sample, that can be used depending on the sample structure. Chemical and electron-impact ionization are often used for gases or vapors whereas electro-sprayionization (ESI) and matrix-assisted laser desorption-ionization (MALDI) are used for liquids and solid biological samples. After ionization the ions are accelerated and deflected in an electromagnetic field and thereby can be separated according to their mass-to-charge ratio. Smaller ions with a high charge deflect stronger than large ions with the same charge. Since organic molecules have a tendency to ionize into one time positively charged ions the charge can often be neglected (Vollhardt, 2005). After separating the ions according to their $\mathrm{m} / \mathrm{z}$ ratio an analyzer catalogs the ions present by selecting a narrow range of $m / z$ or scanning through a range of $\mathrm{m} / \mathrm{z}$. The results are converted into an electric signal that is recorded as a spectrum.

In the context of this thesis, MS-analyses to identify phosphorylation sites in PIP5K2 and PIP5K3 were performed by Dr. Waltraud Schulze (MPI Golm, Germany). Tryptic peptide mixtures were analyzed by LC-MS/MS using a nanoflow Easy-nLC HPLC sytem (Thermo Scientific, Waltham, MA, USA) and an LTQ-Orbitrap (Thermo Scientific, Waltham, MA, USA) hybrid mass spectrometer. Since phosphopeptides only occur as a minor proportion of the total peptides of a protein it was necessary to enrich phosphorylated peptides on a titanium dioxide $\left(\mathrm{TiO}_{2}\right)$ matrix before $\mathrm{MS}$. $\mathrm{TiO}_{2}$ specifically binds negatively charged phospho-serine, phospho-threonine and phospho-tyrosine residues which can then be eluted after several washing steps. The enrichment increases the possibility of detecting phosphorylation sites by reducing the background of highly abundant but not phosphorylated peptides. After MSanalysis the fragmentation spectra obtained are searched against the Arabidopsis protein database. From this database the theoretical masses of trypsin-digested Arabidopsis proteins can be calculated so that the experimentally determined fragment masses can be identified. Simultaneously, the sequence coverage can be calculated, at best representing the complete sequence of the protein of interest. Phosphorylated peptides are identified by screening the database for differences of $96 \mathrm{Da}$, the mass of a phosphorylated group, between experimentally verified and calculated peptide masses.

A freely available tool to find phosphorylation sites in plant proteins is database PhosPhat4.0 (www.cbs.dtu.dk/services/NetPhos/; (Heazlewood et al., 2008)). This database collects phosphorylation sites that were experimentally verified by different research groups during various applications. 


\subsubsection{Detection of phosphorylation sites with PepStar ${ }^{\mathrm{TM}}$ peptide microarrays}

Another approach to identify phosphorylation sites in PI4P 5-kinases was the use of PepStar ${ }^{\mathrm{TM}}$ peptide microarrays (JPT Peptide Technologies GmbH, Berlin, Germany). The basic principle of this method is the use of specific peptides that are immobilized on a matrix which is then subjected to phosphorylation by protein kinases. The protein kinases phosphorylate particular peptides on the matrix depending on their sequence motive. Afterwards phosphorylated peptides are visualized by using different dyes that specifically bind to phosphorlylated peptides, such as ProQDiamond, or by using $y-\left[{ }^{32} \mathrm{P}\right]$ ATP as phosphate donor. Since the sequence and location of each peptide on the peptide array is known, phosphorylated fragments can be analyzed for existing serine, threonine or tyrosine residues.

In the context of this thesis the amino acid sequence of PIP5K2 was split into 15-mer peptides. Each of the synthesized peptides was shifted 4 amino acid residues against the next, resulting in overlapping fragments (Fig. 2.2 A). This is important since one fragment can contain more than one phosphorylation site. By creating overlapping fragments phosphorylated peptides can be compared to not phosphorylated peptides and thus possible phosphorylation sites can be narrowed down. The peptides were then spotted in triplicates onto a glass surface (Fig. 2.2 B) and each peptide array was printed in 3 identical subarrays (Fig. 2.2.C).

A

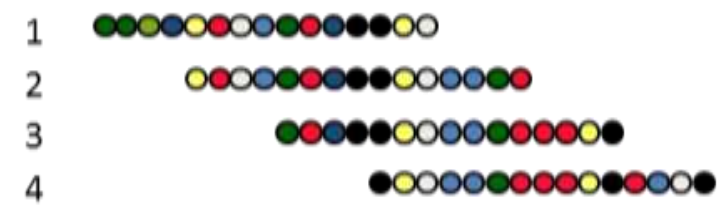

B

C

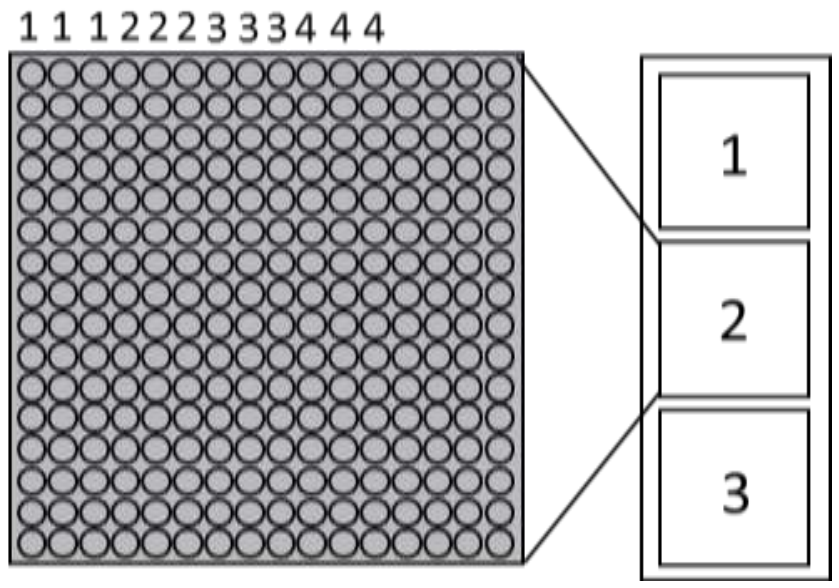

Fig. 2.2: Schematic illustration of a peptide array. A, overlapping peptide fragments are synthesized and spotted in triplicates onto a glass surface (B). Each peptide array consisted of three identical subarrays (C). 
The application was performed according to manufacturer's instructions. In brief two peptide arrays were arranged in a sandwich-like orientation and separated by two spacers, resulting in a total volume of $300 \mu \mathrm{l}$ between the two slides. The peptide arrays were treated with 4 units of PKA (Sigma-Aldrich, Deisenhofen, Germany) or 150-800 $\mu \mathrm{g}$ protein of freshly prepared Arabidopsis extract of 6-week-old plants in a $300 \mu$ reaction containing $100 \mathrm{mM}$ Tris, pH 7.5, $100 \mathrm{mM} \mathrm{MgCl} 2,0.5 \mathrm{M} \mathrm{NaCl}, 1 \mathrm{mM}$ EGTA, $1 \mathrm{mM}$ DTT, phosphatase inhibitor cocktail (PhosSTOP, Roche Diagnostics GmBH, Mannheim, Germany), $10 \mu \mathrm{M}$ ATP and $200 \mu \mathrm{Ci} \psi$ - $\left[{ }^{32} \mathrm{P}\right]$ ATP (Hartmann Analytics, Braunschweig, Germany) by pipetting the reaction mixture in between the two slides. The "microarray sandwich" was placed into an incubation chamber composed of a wet cloth placed in a large Petri-dish to avoid evaporation and incubated for $1 \mathrm{~h}$ at room temperature. After incubation the peptide arrays were washed 3 times with $2 \%(\mathrm{v} / \mathrm{v})$ phosphoric acid, dd $\mathrm{H}_{2} \mathrm{O}$ and methanol respectively. Each washing step was performed for 20 min after which the peptide arrays were dried and phosphorylated spots visualized by exposing an imager screen for 1 day in the case of PKA treatment or 7 days in the case of treatment with plant extract. Phosphorylated peptides were identified with a peptide map included in the kit. By comparing phosphorylated peptides to non-phosphorylated neighbour fragments, phosphorylation sites could be identified.

\subsubsection{In silico analysis of putative phosphorylation sites}

Another approach to identify possible phosphorylation sites is the use of bioinformatical analyses that predict phosphorylation sites using specific algorithms (Blom et al., 1999). Various algorithms are available for several posttranslational modifications both in eukaryotic and prokaryotic organisms. In this thesis NetPhos 2.0 (CBS Prediction Center, Center For Biological Sequence Analysis, TU Denmark, http://www.cbs.dtu.dk/services/NetPhos) was used. It must be noted that most algorithms, including NetPhos 2.0, are not adapted to plant specific phosphorylation sites. Therefore, the prediction of phosphorylation sites in plant proteins with web-accessible algorithms is error-prone and hard to interpret.

\subsection{Site-directed mutagenesis}

The effects of phosphorylation on a target protein are largely due to the change in the surface charge introduced by the anionic phosphate-moiety. The effects of possible phosphorylation sites can thus be characterized in vitro and in vivo by constitutively mimicking phosphorylation or dephosphorylation via the introduction of specific charged or non-charged residues in the 
respective positions of a protein (Tang et al., 2003; Tang et al., 2005). Figure 2.3 illustrates the phosphorylation of serine as an example. In the dephosphorylated state the hydroxyl group of the serine residue is free and uncharged (Fig. 2.3 A). Upon phosphorylation, the hydroxyl group anchors the phosphate group which introduces a 2-fold negative charge (Fig. $2.3 \mathrm{~B}$ ). Through the surface charge that is introduced protein phosphorylation can not only effect conformational changes but also influence electrostatical interactions of the protein. Altering a putative phosphorylation site to alanine (A) resembles the uncharged hydroxyl group (Fig. 2.3 C) and thus mimics the dephosphorylated state of the protein (phosphoablation). The particular phosphorylation site is not accessible to phosphorylation any longer due to the missing hydroxyl group. In contrast, altering the phosphorylation site to aspartate (D) mimics the phosphorylated state of the protein (phosphomimicry) since $D$ is introducing a permanent negative charge into the protein structure (Fig. $2.3 \mathrm{D}$ )
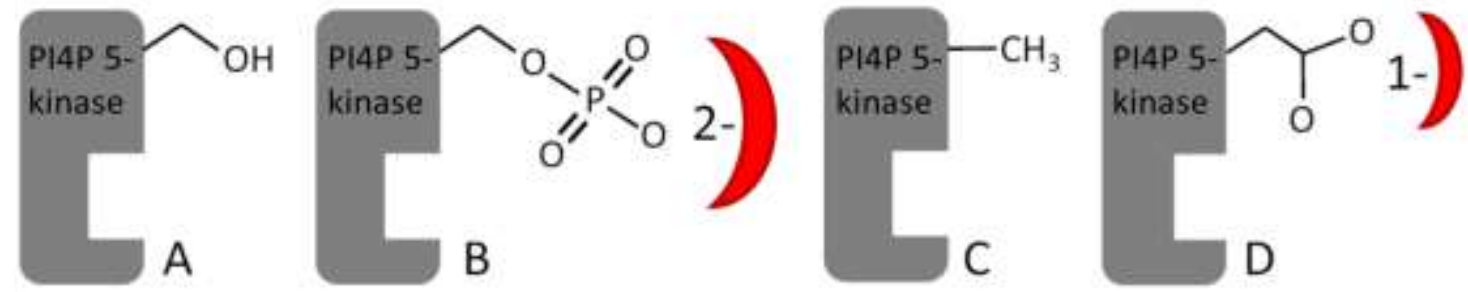

Fig. 2.3. Phosphomimicry and phosphoablation of known phosphorylation sites in PI4P 5-kinases. Serine was chosen as example to illustrate effects of mutation on known phosphorylation sites (A). B, phosphorylation of serine introduces two negative charges into the PIP5K structure. C, altering the serine residue to alanine resembles constitutive dephosphorylation. $\mathrm{D}$, altering the site to aspartate also introduces a negative charge and thus mimics the phosphorylated state.

The specific exchange of amino acids in proteins was achieved by site-directed mutagenesis using QuickChange ${ }^{\mathrm{TM}}$ technology (Stratagene, La Jolla, CA, USA). This method is based on the polymerase-chain-reaction (PCR)-amplification of a complete plasmid with primers that contain the desired base pair exchange encoding the altered amino acid. The PCR was performed with Phusion High fidelity polymerase (NEB, Ipswich, MA, USA) in a $50 \mu$ l reaction according to manufacturer's instructions. The amplification was achieved using the following thermal cycling steps: $98^{\circ} \mathrm{C}$ for $30 \mathrm{~s}$ as initial DNA melting step, 25 cycles of $98^{\circ} \mathrm{C}$ for $10 \mathrm{~s}, 55$ ${ }^{\circ} \mathrm{C}$ for $30 \mathrm{~s}, 30 \mathrm{~s} /$ base pair at $72{ }^{\circ} \mathrm{C}$. The final elongation was performed at $72{ }^{\circ} \mathrm{C}$ for $3 \mathrm{~min}$.

Alternatively, Pfu DNA polymerase (MBI Fermentas, St. Leon Rot, Germany) was used in a total volume of $50 \mu \mathrm{l}$ according to manufacturer's instructions. Thermal cycling was performed as described for the Phusion High fidelity polymerase with the exception that the denaturation step was carried out at $95^{\circ} \mathrm{C}$ and the elongation was allowed $2 \mathrm{~min} /$ base pair.

After amplification the reaction was restricted with $10 \mathrm{U}$ of Dpnl (MBI Fermentas, St. Leon Rot, Germany) at $37{ }^{\circ} \mathrm{C}$ over night to degrade methylated template DNA. The unmethylated 
product DNA was used to transform chemically-competent E. coli as described above. A complete list of the oligonucleotide sequences used is recorded in the appendix in Table 7.1.

\subsubsection{DNA sequencing}

To verify point mutations in plasmids DNA was sequenced by GATC Biotech (Konstanz, Germany) and LGC genomics (Berlin, Germany) according to manufacturer's instructions.

\subsection{Preparation of constructs for the transformation of $A$. thaliana}

For the transformation of pip $5 k 1$ pip $5 k 2$ double mutant plants and pip $5 k 3$ single mutant plants pCAMBIA $33.00 \mathrm{GC}$ constructs were created using the GATEWAY system. The PCAMBIA vector contained a 1500-bp PIP5K2 promoter fragment as fusion to a C-terminal EYFP for the transformation pip $5 k 1$ pip $5 k 2$ double mutant plants. For the transformation of pip $5 k 3$ single mutant plants a pCAMBIA 33.00 GC construct with the respective 1500-bp PIP5K3 promotor fragment as fusion to an N-terminal EYFP was used. Both pCAMBIA $33.00 \mathrm{GC}$ constructs contained a Gateway cassette with attR1 and attR2 sites necessary for transfering DNA fragments between donor and target vectors. pLATGW constructs containing the appropriate PIP5K2-CDNA and PIP5K3-cDNA were used as donor vectors, respectively. The Gateway reaction was performed using $10 \mathrm{fmol}$ donor vector, $20 \mathrm{fmol}$ pCAMBIA $33.00 \mathrm{GC}$ and $1 \mu \mathrm{l}$ Clonase II (Invitrogen Karlsruhe, Germany) in a total reaction of $10 \mu$ l containing $7 \mu$ l TE-buffer (10 mM Tris, pH 8, 1 mM EDTA). The samples were incubated at room temperature for $16 \mathrm{~h}$. The reaction was stopped by adding $2 \mu \mathrm{g}$ of protein kinase K (Invitrogen, Karlsruhe, Germany) and incubating the sample for $10 \mathrm{~min}$ at $37^{\circ} \mathrm{C}$.

\subsection{Electrophoretic separation of DNA mixtures}

DNA was mixed with $1 / 5$ sample buffer ( $60 \%(\mathrm{v} / \mathrm{v})$ glycerol, $0.4 \%$ orange $\mathrm{G}$ ) and separated on agarose gels (40 mM Tris HCl, pH 8.3, 2 mM EDTA solidified with 1-1.5 \% (w/v) agarose) for 20$30 \mathrm{~min}$ at $120 \mathrm{~V}$. The DNA fragment size was estimated by running $5 \mu \mathrm{l}$ of GeneRuler $1 \mathrm{~kb}$ DNA Ladder (MBI Fermentas, St. Leon Rot, Germany) on the same gel. The separated DNA fragments were visualized by an UV imager (INTAS Gel IX Imager, Sartorius, Göttingen, Germany) after staining the gel in a $2 \mu \mathrm{g} / \mathrm{ml}$ ethidium bromide bath for $10 \mathrm{~min}$. 


\subsection{Preparation of chemically-competent Agrobacterium tumefaciens (Agrobac. tumefaciens) cells}

For transformation of $A$. thaliana plants the Agrobac. tumefaciens strain EH 105 (Hood et al., 1993) was used. To render the cells competent for transformation a colony was picked from a plate and precultured in $2 \mathrm{ml}$ of YEB medium $(0.5 \%(\mathrm{w} / \mathrm{v})$ beef extract, $0.1 \%(\mathrm{w} / \mathrm{v})$ yeast extract, $0.5 \%(\mathrm{w} / \mathrm{v})$ peptone, $0.5 \%(\mathrm{w} / \mathrm{v})$ sucrose, $\left.5 \mathrm{mM} \mathrm{MgSO}_{4}\right)$ containing $50 \mathrm{\mu g} / \mathrm{ml}$ rifampicin at $28{ }^{\circ} \mathrm{C}$ overnight. The preculture was used to inoculate $50 \mathrm{ml}$ of YEB medium containing rifampicin (as above) and grown at $28^{\circ} \mathrm{C}$ until an $\mathrm{OD}_{600}$ of 0.5 was reached. The cells were collected by centrifugation at $5000 \times \mathrm{g}$ and $4{ }^{\circ} \mathrm{C}$ for $5 \mathrm{~min}$ and washed with $10 \mathrm{ml}$ of ice cold $0.15 \mathrm{M} \mathrm{NaCl}$ solution. The centrifugation step was repeated, the cells were resuspended in $1 \mathrm{ml}$ of ice-cold $75 \mathrm{mM} \mathrm{CaCl}_{2}$ solution, aliquoted and snap-frozen in liquid nitrogen. Cells were stored at $-80^{\circ} \mathrm{C}$ for further experiments.

\subsection{Transformation of chemically-competent Agrobac. tumefaciens cells}

Agrobac. tumefaciens cells were transformed according to (Hofgen and Willmitzer, 1988). $50 \mu \mathrm{l}$ of competent Agrobacterium cells were thawed and incubated with $5 \mu$ plasmid DNA for $5 \mathrm{~min}$. After incubation, the cells were shock-frozen in liquid nitrogen, incubated at $37{ }^{\circ} \mathrm{C}$ for $5 \mathrm{~min}$ and $1 \mathrm{ml}$ of YEB medium was added. The cells were then cultured at $28^{\circ} \mathrm{C}$ for $4 \mathrm{~h}$ and plated on solidified YEB medium (as above solidified with $1.5 \%(\mathrm{w} / \mathrm{v})$ agar) containing $50 \mu \mathrm{g} / \mathrm{ml} \mathrm{rifampicin} \mathrm{and} \mathrm{the} \mathrm{respective} \mathrm{antibiotic} \mathrm{for} \mathrm{the} \mathrm{selection} \mathrm{of} \mathrm{positive} \mathrm{transformants.}$

\subsection{Transformation of $A$. thaliana}

The Agrobacterium-mediated transformation of recombinant constructs into Arabidopsis plants was performed using the floral dip method (Clough and Bent, 1998). Transformed Agrobacterium cells were picked from plates, transferred to $5 \mathrm{ml}$ of YEB medium containing the appropriate antibiotics for selection and grown at $28{ }^{\circ} \mathrm{C}$ over night. The precultures were used to inoculate $300 \mathrm{ml}$ of YEB medium. After cultivation at $28{ }^{\circ} \mathrm{C}$ for $12-16 \mathrm{~h}$, the cell suspension was centrifuged at $10000 \times \mathrm{g}$ for $20 \mathrm{~min}$ and the supernatant was discarded. The collected cells were washed with $10 \mathrm{ml} 5 \%(\mathrm{w} / \mathrm{v})$ sucrose, sedimented and resuspended in a total volume of $400 \mathrm{ml}$ of $5 \%$ (w/v) sucrose including $0.025 \%$ (v/v) Silwet (OSi Specialties Inc., South Charleston, WI, USA) to increase dispersion and adhesion of the cell-sucrose mixture to the plants. Arabidopsis plants in their flowering stage were dipped in the Agrobacterium cell 
suspension. If only few flowers were present, the procedure was repeated after one week. The transformed plants were grown under greenhouse conditions and their offspring was selected for BASTA resistance by spraying 7 to 14-days-old plants with $0.5 \%$ (v/v) BASTA solution (Bayer crop science, Mannheim, Germany). Resistant seedlings were isolated and T2 seeds were used for further analysis.

One aim of this thesis was the test for in vivo functionality of several PIP5K2 and PIP5K3 variants by transforming different plant lines with the respective PIP5K2 CDNA and PIP5K3 CDNA (c.f. 2.13).

PIP5K2 cDNA was expressed in the Arabidopsis pip5k1 pip5k2 double mutant background. Arabidopsis PIP5K1- and PIP5K2-alleles in these plants are interrupted by T-DNA insertions resulting in a knock-out of both genes. pip5k1 pip5k2 double mutant plants have an easy recognizable dwarfism phenotype and that can be complemented by the expression of a functional PIP5K2 (Fig. 2.4 D). pip5k1 pip5k2 double mutants also depict male sterility, therefore it is not possible to transform these plants. Instead, heterozygous PIP5K1 pip5k1 $\mathrm{x}$ pip5k2 pip5k2 mutants that have no obvious phenotype (Fig. 2.4 E) were used for transformation experiments and had to be selected before transformation. After transformation with the respective PIP5K2-CDNA, PIP5K1 pip5k1 x pip5k2 pip5k2 mutant T2 seeds were sown and selected for pip $5 k 1$ pip $5 k 2$ mutant plants containing the respective PIP5K2-cDNA. These plants were then analysed for their ability to complement the dwarfism phenotype.

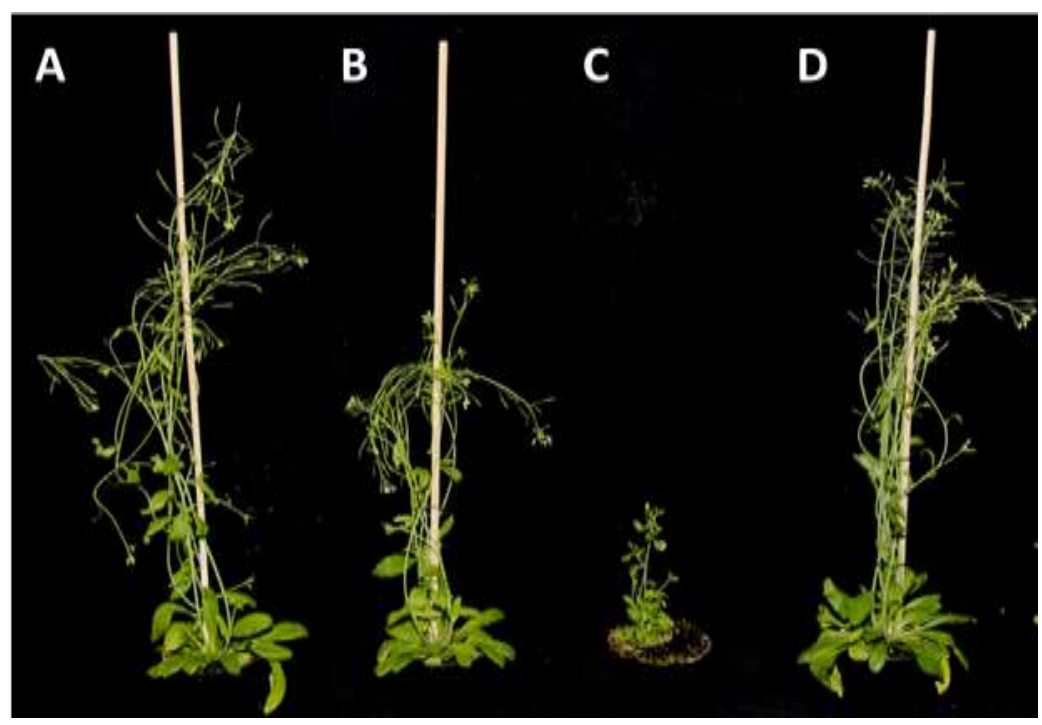

Fig. 2.4. Arabidopsis pip5k1 pip5k2 double mutants have a serve phenotype. Plants were grown for 6 weeks under long day conditions. A, Arabidopsis wild type. B, Arabidopsis pip5k1 mutant. C, Arabidopsis pip5k1 pip5k2 double mutant. D, complemented pip5k1 pip5k2 double mutant with PIP5K2. Images were kindly provided by Dr. Irene Stenzel. 
PIP5K3 c-DNA was expressed in the Arabidopsis pip5k3 mutant background. In these plants PIP5K3-alleles are knocked-out by T-DNA insertions. Arabidopsis pip5k3 mutants are affected in root hair growth (Stenzel et al., 2008) but are fertile and therefore were directly used for transformation experiments.

\subsection{Isolation of genomic DNA from Arabidopsis plants}

Genomic DNA was isolated from medium-sized leaves of 2-weeks-old Arabidopsis plants, which were harvested and shock frozen in liquid nitrogen. The plant tissue was ground with glass beads in a type MM200 shaking mill (Retsch, Haan, Germany) and the pulverized tissue was thawed in $250 \mu \mathrm{l}$ of CTAB ( $2 \%$ (w/v) CTAB, $100 \mathrm{mM}$ Tris HCl, pH 8.0, $20 \mathrm{mM}$ EDTA, pH 8.0, 1.4 M NaCl). After incubation at $65^{\circ} \mathrm{C}$ for $10-20 \mathrm{~min}$, the samples were cooled and an equal volume of chloroform:isoamyl alcohol (24:1) was added. After agitation, the samples were centrifuged at $7500 \times \mathrm{g}$ for $3 \mathrm{~min}$ at room temperature and $200 \mu \mathrm{l}$ of the upper aqueous phase were transferred into a new reaction tube. Then $20 \mu \mathrm{l}$ of $\mathrm{CTAB} / \mathrm{NaCl}$ solution ( $10 \% \mathrm{CTAB}, 0.7 \%$ $\mathrm{NaCl}$, preheated to $65^{\circ} \mathrm{C}$ ) were added and the samples were mixed and incubated for $2 \mathrm{~min}$ at room temperature. Afterwards an equal volume of isopropanol was added, the samples were mixed by inverting the tubes several times and incubated at room temperature for $2 \mathrm{~min}$. To precipitate the plasmid DNA, samples were spun at $20000 \mathrm{xg}$ for $10 \mathrm{~min}$. The supernatant was carefully decanted, the pellet was washed with $100 \mu \mathrm{l}$ of $75 \%(\mathrm{v} / \mathrm{v})$ ethanol and spun at 16000 $\mathrm{x} g$ for $15 \mathrm{~min}$. Finally the ethanol was removed, the pellet was dried and dissolved over night in $50 \mu$ l of dd $\mathrm{H}_{2} \mathrm{O}$.

\subsection{Identification of T-DNA mutants}

Successful transformation of Arabidopsis plants was tested by amplifying specific DNA sequences using PCR. Arabidopsis wild type plants and T-DNA insertion mutants were genotyped by using $1 \mu \mathrm{l}$ of genomic DNA as a template. The PCR was performed with Hot TaqDNA Polymerase (Peqlab, Erlangen, Germany) according to manufacturer's instructions in a total volume of $20 \mu \mathrm{l}$. The following thermal cycling was used: $3 \mathrm{~min}$ at $94{ }^{\circ} \mathrm{C}$ as initial DNA melting step following $27 \mathrm{cycles}$ at $94^{\circ} \mathrm{C}$ for $30 \mathrm{~s}, 58^{\circ} \mathrm{C}$ for $30 \mathrm{~s}$ and $72{ }^{\circ} \mathrm{C}$ for $2 \mathrm{~min}$. The final elongation was performed at $72{ }^{\circ} \mathrm{C}$ for $3 \mathrm{~min}$. To confirm wild type pip5k1 alleles the primer combination P1 and P2 were used. For the identification of pip5k1 T-DNA insertion lines the primer combination P2 and P7were used. To confirm pip5k2 wild type alleles the primer combinations $\mathrm{P} 3$ and $\mathrm{P} 4$ were used. pip5k2 T-DNA insertions were amplified using the primer 
combinations P4 and P7. Wild type pip5k3 alleles were confirmed using the primer combination P5 and P6. For the identification of pip $5 k 3$ T-DNA insertion lines the primer combinations P6 and P7 were used. To confirm extopic insertion of PIP5K3::EYFP:PIP5K3 variants in the pip5k3 mutant background the primer combinations $\mathrm{P} 8$ and $\mathrm{P} 9$ were used.

\subsection{Visual examination of root hairs}

To analyse root hair phenotypes, seeds of control plants (wild type and pip5k3 mutants) and transformed plants were sterilized, sown on rectangular Petri-dishes containing MS growth medium, and cultured vertically under continuous light. Root hair phenotypes were examined after one week of growth by microscopy (see section 2.22). The root hair length was measured with imageJ (http://rsbweb.nih.gov/ij/).

\subsection{Transient expression of fluorescence-labled proteins in tobacco pollen tubes}

Tobacco plants were grown on soil (Type Substrate 1, Klasmann-Deilmann GmBH, Geeste, Germany) in the green house under long day conditions with $16 \mathrm{~h}$ of light ( $\sim 130-150 \mu \mathrm{mol}$ photons $\mathrm{m}^{-2} \mathrm{~s}^{-1}$ ). Tobacco pollen was transformed by ballistic bombardment of the plant material with DNA-coated gold particles. In brief, plasmid DNA was precipitated on $1.25 \mathrm{mg}$ gold particles ( $1 \mu \mathrm{m}$, Bio Rad, Munich, Germany) by adding $1 \mathrm{M} \mathrm{CaCl}_{2}$ and $16 \mu \mathrm{M}$ spermidine. After mixing the samples for 2 min, gold particles were washed with $95 \%$ ethanol for 3 times. Mature pollen was harvested from 3 tobacco flowers of 8 week old plants and resuspended in $6 \mathrm{ml}$ of growth medium (5\% (w/v) sucrose, $12.5 \%(\mathrm{w} / \mathrm{v})$ PEG-6000, $0.03 \%(\mathrm{w} / \mathrm{v})$ casein hydrolysate, $15 \mathrm{mM}$ MES KOH, pH 5.9, $1 \mathrm{mM} \mathrm{CaCl}, 1 \mathrm{mM} \mathrm{KCl}, 0.8 \mathrm{mM} \mathrm{MgSO}_{4}, 1.6 \mathrm{mM} \mathrm{H}_{3} \mathrm{BO}_{3}$, $30 \mu \mathrm{M} \mathrm{CuSO}_{4}, 10 \mu \mathrm{g} / \mathrm{ml}$ rifampicin; according to Read et al. (1993). Resuspended pollen was filtered onto cellulose acetate filters (Sartorius, Göttingen, Germany) and placed on filter paper (Whatman, Maidstone, UK) soaked with growth medium. Pollen was immediately bombarded with plasmid coated gold particles via a helium-driven particle accelerator (PDS1000/He, Biorad, Munich, Germany) using 1350 psi rupture discs and a vacuum of 28 inches of mercury according to manufacturer's instructions. After bombardment, pollen grains were resuspended in growth medium and pollen tubes grown in small droplets of media directly on microscope slides. To analyse localization of the transformed constructs pollen were grown for $7 \mathrm{~h}$. The investigation of pollen tube morphology was carried out after 12-16 h of growth. 


\subsection{Microscopy}

In the context of this thesis PIP5K2 and PIP5K3 proteins fused to an enhanced yellowfluorescent protein (EYFP) were investigated for their localization in tobacco pollen tubes and their effects on tobacco pollen tube morphology. Yellow-fluroescent protein (YFP) is a derivative of the green fluorescent protein (GFP) which was first identified in the jellyfish Aequorea victoria (Shimomura et al., 1962). GFP is a fluorophor that builds spontaneously without the necessity of catalytic enzyme activity (Ormo et al., 1996). Mutation of particular amino acid residues in GFP led to the development of several other fluorescent proteins like YFP or red-fluorescent protein (RFP). EYFP is derivative of YFP and is characterized by a brighter fluorescence and a higher stability (Janke et al., 2004).

The effects of PIP5K2:EYFP and EYFP:PIP5K3 on pollen tube morphology and the effects of EYFP:PIP5K3 on root hair development were investigated with the Fluorescence Stereo Microscope AXIO IMAGER M1 (Carl Zeiss, Jena, Germany) using an AXIO 46 HE filterset for YFP and a $200 \times$ magnification. Images were taken with SteREO Discovery V8 (Carl Zeiss, Jena, Germany). Root hair length, pollen tube diameter and fluorescence intensities were measured with Image J (http://rsbweb.nih.gov/ij/).

The localization of PIP5K2:EYFP and EYFP:PIP5K3 in tobacco pollen tubes was investigated with the confocal microscope LSM 510 Meta (Carl Zeiss, Jena, Germany). EYFP was excited with an $\mathrm{Ar}^{+}$-Laser and a wavelength of $488 \mathrm{~nm}$. Emitted fluorescence was detected with a HTF 405/488/514/633 major beam spiltter and a 505-530nm band pass filters. Images were taken with corresponding software programs (Zeiss LSM Image Browser version 4.0, Carl Zeiss, Jena, Germany). 


\section{RESULTS}

Ptdlns $(4,5) \mathrm{P}_{2}$ mediates various important cellular functions and its production by PI4P 5 kinases needs to be tightly controlled. Previous experiments using different plant model systems have shown that Ptdlns $(4,5) \mathrm{P}_{2}$ production and PI4P 5-kinase activity can increase very rapidly after stress. This suggests an efficient regulatory mechanism for the activation of PI4P 5-kinases. Although several correlative investigations towards the dynamics $\operatorname{Ptd} \operatorname{lns}(4,5) \mathrm{P}_{2}$ formation upon stress-treatments or during development have previously been conducted, the molecular mechanisms controlling rapid changes in PI4P 5-kinase-activity are not clear. It is the working hypothesis of this thesis that posttranslational modification plays an important role in controlling PI4P 5-kinase activity. In support of this concept there is published experimental evidence that Arabidopsis PIP5K1 can be phosphorylated in vitro and it was the aim of this work to verify whether phosphorylation of other PI4P 5-kinases occurs in plants, to determine which amino acids are phosphorylated and what consequences of phosphorylation are regarding localization and functionality of the enzymes in plants. Two PI4P 5-kinases from Arabidopsis were selected (PIP5K2 and PIP5K3) for further characterization. PIP5K2 was chosen because it is ubiquitously expressed and a possible candidate for a stress-induced PI4P 5kinase, while PIP5K3 was selected as an example for an enzyme with root-specific expression, possibly displaying regulation by a mode different from that of PIP5K2.

\subsection{PIP5K2 can be recombinantly expressed and purified in E. coli}

To test phosphorylation of Arabidopsis PIP5K2, the enzyme was recombinantly expressed in $E$. coli. E. coli is a good expression system for PI4P 5-kinases as it is a prokaryote not exhibiting phosphoinositide signalling and lacks PI4P 5-kinase activity. Therefore, the activity of expressed PI4P 5-kinases can be analysed by using crude extracts without additional purification of the protein (Stenzel et al., 2008). In order to aid interpretation of in vitro modification patterns obtained with the recombinant proteins and to enable MS-analysis, PIP5K2 was purified.

PIP5K2 was cloned into a pETM41 expression vector to be expressed as a fusion protein to an $\mathrm{N}$-terminal MBP tag which enhances the solubility of the protein. The pETM41 MBP-PIP5K2 construct was kindly provided by Dr. Irene Stenzel (MLU Halle-Wittenberg). MBP-PIP5K2 was expressed in E. coli Rosetta 2 cells at $28{ }^{\circ} \mathrm{C}$ for $4 \mathrm{~h}$ (for details see section 2.10.4). After 
harvesting and disruption of the cells, crude cell extract was applied to further purification by affinity chromatography via an MBP trap matrix (GE Healthcare Life Sciences, Stockholm, Sweden). To analyse enrichment of MBP-PIP5K2, each purification step was monitored by a Coomassie stained SDS-PAGE (Fig. 3.1 A). As depicted in Figure 3.1 A the number of protein bands was reduced with subsequent purification steps. After the first elution step a band with a size of $120 \mathrm{kDa}$ appeared, which was not as pronounced in the crude extract or the wash fraction. Other smaller bands were only slightly visible. In the second elution fraction the same band was detected even stronger, at the same time the amount of smaller bands up to a size of around $40 \mathrm{kDa}$ increased compared to the first elution fraction. As the full-length MBPPIP5K2 fusion is about $120 \mathrm{kDa}$ in size, it is possible that the emerging band in both elution fractions was the expressed PI4P 5-kinase. The identity of the $120 \mathrm{kDa}$ band was verified by immunoblotting using an MBP antibody that specifically recognizes the MBP-tag of the MBPPIP5K2 fusion protein. For the immunoblot (Fig. 3.1 B) protein samples were loaded in the same order shown for Figure 3.1 A. In contrast to the decrease of protein bands discovered after unspecific Coomassie staining, Figure 3.1 B indicates that the amount of specifically detected protein bands by the MBP antibody increased with each purification step. Next to several smaller bands a protein band with a size of $120 \mathrm{kDa}$ appeared in the first and second eluted fractions similar to the pattern detected with Coomassie staining.

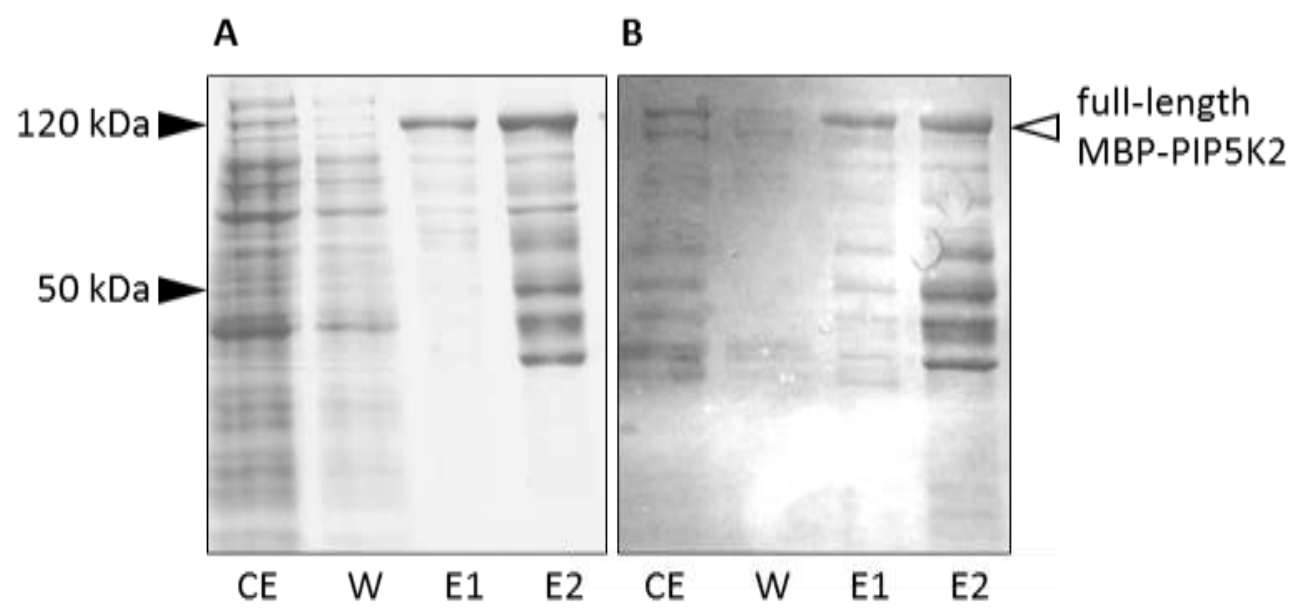

Fig. 3.1.: Purification of MBP-PIP5K2 by affinity chromatography. MBP-PIP5K2 was heterologously expressed in $E$. coli and purified with an MBP trap column. The progress of purification was documented by a Coomassie-stained SDS-PAGE-gel (A) and verified by immunodetection using an MBP antibody (B). $C E$, crude extract; W, wash; E1, eluted fraction 1; E2, eluted fraction 2. Black arrowheads indicate protein sizes defined by a commercial protein marker and apply for A and B; white arrowheads show the size of the full-length MBP-PIP5K2 fusion protein. The experiment was repeated twice with similar results.

To confirm successful purification and to document that enriched MBP-PIP5K2 was active, equal protein amounts of crude extract and of the second eluted fraction were subjected to radiolabeled activity tests. Figure 3.2 illustrates the specific activities of the selected fractions 
as the amount of PtdIns $(4,5)_{2}$ synthesized in pmol per minute per mg protein. The second eluted fraction was approximately five times more active than the crude extract. Purified MBP was also tested for its ability to synthesize PtdIns(4,5) $\mathrm{P}_{2}$ but no activity was detected (Fig. 3.2).



Fig. 3.2: Specific activities of recombinantly expressed MBP-PIP5K2. Crude extracts (CE) and the second eluted fraction (E2) were incubated with commercially available PtdIns4P and $y-\left[{ }^{32} \mathrm{P}\right]$ ATP. Product lipids were extracted and separated by TLC. The amount of radiolabled PtdIns $(4,5) \mathrm{P}_{2}$ was quantified by a phosphorimager. The purified maltose binding protein (MBP) alone was used as a negative control. Data represent means \pm standard deviation (SD) of two independent experiments.

The specific activity of crude extract and second eluted fraction were compared with the determined protein amounts of each fraction as shown in Table 3.1. Although a 10-fold decrease of both protein concentration and overall activity during purification was detected, the specific activity increased. Based on these results a purification factor of about 4.6 was calculated. The recombinantly expressed and purified MBP-PIP5K2 was then applied to further biochemical investigations as will be described in the next sections.

Table 3.1: Purification table of enriched PIP5K2 via affinity chromatography. Data show the progress of purification by comparing the specific activity of crude extract with the specific activity of the second eluted fraction.

\begin{tabular}{ccccccc}
\hline Step & $\begin{array}{c}\text { Volume } \\
(\mathbf{m l})\end{array}$ & $\begin{array}{c}\text { Protein } \\
(\mathbf{m g})\end{array}$ & $\begin{array}{c}\text { Activity } \\
(\mathbf{p m o l} / \mathbf{m i n})\end{array}$ & $\begin{array}{c}\text { Specific activity } \\
\text { (pmol/min/mg) }\end{array}$ & $\begin{array}{c}\text { Yield } \\
\text { (\%) }\end{array}$ & $\begin{array}{c}\text { Purification } \\
\text { (factor) }\end{array}$ \\
\hline $\begin{array}{c}\text { Crude } \\
\text { Extract }\end{array}$ & 10 & 64.6 & 147.2 & 2.3 & 100 & 1 \\
Elution 2 & 1 & 0.75 & 7.95 & 10.6 & 5.4 & 4.6 \\
\hline
\end{tabular}

\subsection{Phosphorylation of recombinantly expressed and purified PIP5K2}

In previous experiments (Westergren et al., 2001) it was demonstrated that Arabidopsis PIP5K1 can be phosphorylated by commercially available PKA. PIP5K1 is similar in sequence to PIP5K2 and the two enzymes show $89 \%$ identity of the amino acid level. To test whether 
homologous PIP5K2 can also be subjected to phosphorylation and to establish a working experimental set up, purified MBP-PIP5K2 was first incubated with PKA.

\subsubsection{Recombinant PIP5K2 is phosphorylated by PKA in vitro}

Phosphorylation of MBP-PIP5K2 was tested in vitro by incubating $5 \mu \mathrm{g}$ of recombinantly expressed and purified protein with PKA and radiolabeled $y-\left[{ }^{32} \mathrm{P}\right]$ ATP. After incubating the samples for $1 \mathrm{~h}$, proteins were separated via SDS-PAGE and stained with Coomassie. Protein phosphorylation was detected by exposing a radiosensitive film.

Figure 3.3 shows a section of a Coomassie-stained gel $(A)$ as a loading control and the corresponding autoradiograph (B). Phosphorylation by PKA was detectable for the MBP-PIP5K2 full-length protein (Fig. 3.3 B, lane 1, white arrowhead), whereas no phosphorylation was discovered in the absence of exogenous PKA (Fig. 3.3 B lane 4). To exclude that the detected protein phosphorylation can be attributed to another, co-migrating protein or to phosphorylation of the MBP-tag, the tag was cleaved off by a TEV protease (Fig 3.3, lane 2). The resulting PIP5K2 fragment was smaller, as indicated by the black arrowhead, but still phosphorylated. The reversibility of the phosphorylation was verified by incubating PKAtreated MBP-PIP5K2 with SAP. As shown in Figure $3.3 \mathrm{~B}$ lane 3 phosphorylated protein bands disappeared after SAP-treatment, suggesting that phosphorylation of PIP5K2 depended on an enzymatic reaction and was not caused by mere binding of radiolabeled ATP to positively charged regions of the protein.

A

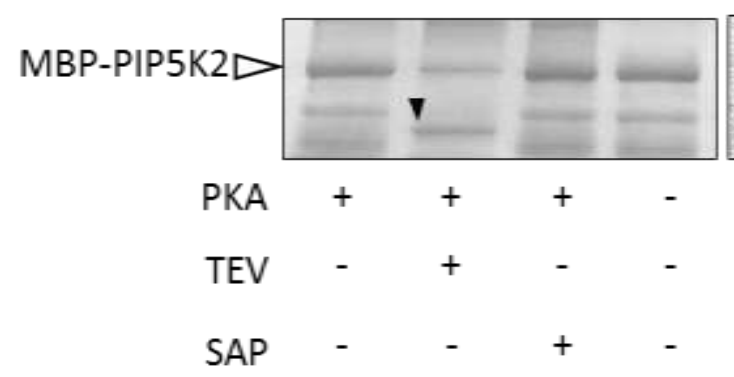

B
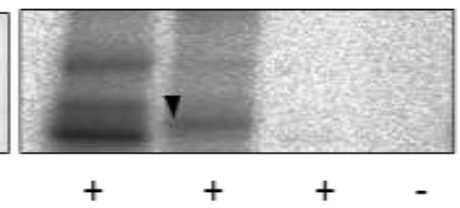

$-\quad+$

Fig. 3.3: Recombinantly expressed MBP-PIP5K2 is phosphorylated by PKA. Equal amounts of purified MBP-PIP5K2 were incubated with PKA and $y-\left[{ }^{32}\right.$ P] ATP. TEV and SAP were added as indicated. Samples were denaturated, separated by SDS-PAGE $(A)$ and phosphorylated bands were visualized by a phosphorimager (B). The white arrowhead indicates the size of full-length MBP-PIP5K2 fusion protein. Black arrowheads indicate the size of the smaller PIP5K2 fragment after treatment with TEV protease. Data are representatives for two independent experiments.

The data so far suggest that Arabidopsis PIP5K2 can be phosphorylated by PKA in vitro. PKA belongs to the mammalian AGC kinases which represent a large protein kinase family. Although AGC kinases are also present in plants, little is known about their function (Robert 
and Offringa, 2008) and no direct PKA homologue has been found to be encoded in the Arabidopsis genome. To test whether PIP5K2 can also be regulated through phosphorylation by an endogenous Arabidopsis protein kinase, subcellular fractions of Arabidopsis plants were investigated for their ability to phosphorylate MBP-PIP5K2, as will be described in the next section.

\subsubsection{Recombinant PIP5K2 is phoshorylated by an endogenous Arabidopsis protein kinase}

As PIP5K2 is ubiquitously expressed in Arabidopsis, extracts from leaves of 6-week-old plants were used as a source for protein kinase activity. To test for phosphorylation, different amounts of purified MBP-PIP5K2 were incubated with Arabidopsis leaf extract and radiolabeled $\left.\mathrm{Y}-{ }^{32} \mathrm{P}\right]$ ATP. After incubation for $1 \mathrm{~h}$, samples were separated by SDS-PAGE and the extent of $\mathrm{P}^{32}$-incorporation was visualized by a phosphorimager (Fig. 3.4 A). Figure 3.4 A indicates, that leaf extract phosphorylated a protein at $120 \mathrm{kDa}$ (lane 1), which is not visible when leaf extract alone was applied (Fig. 3.4 A lane 6), suggesting that the $120 \mathrm{kDa}$ sized band represents phosphorylated full-length MBP-PIP5K2. This suggestion is supported by the observation that the signal at $120 \mathrm{kDa}$ intensified when increasing amounts of leaf extracttreated MBP-PIP5K2 were loaded (lanes 3 and 5).

A

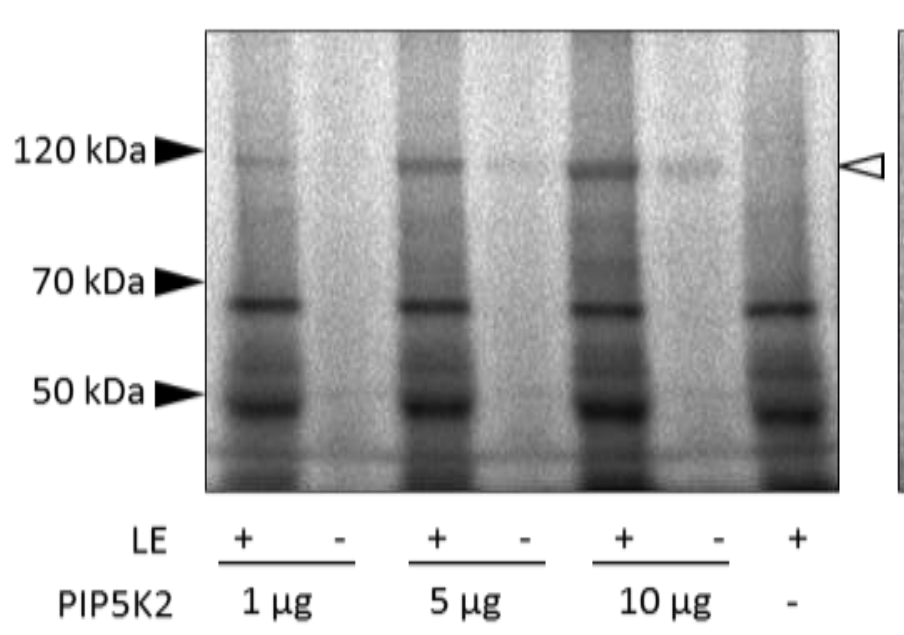

B

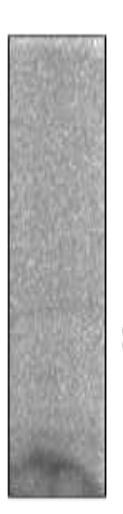

$95^{\circ} \mathrm{C}$

LE

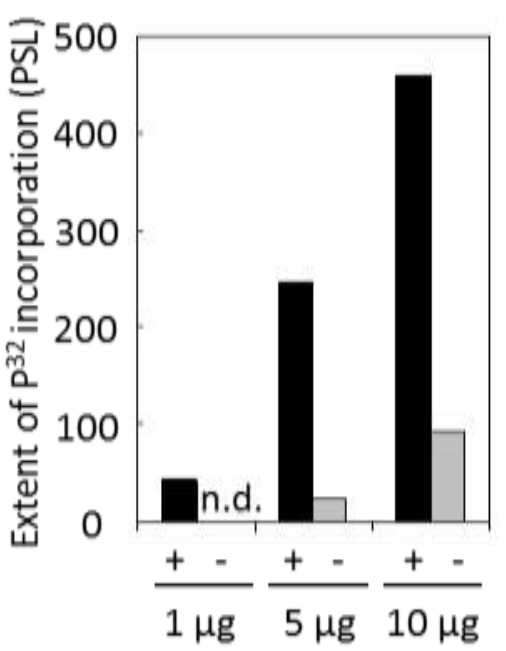

Fig. 3.4: Recombinantly expressed MBP-PIP5K2 is phosphorylated by an Arabidopsis protein kinase present in leaf extract. Recombinant MBP-PIP5K2 was incubated with (+) or without (-) leaf extract (LE). LE (lane 7) and denaturated LE $\left(95^{\circ} \mathrm{C}\right.$ LE) incubated with $\mathrm{Y}-\left[{ }^{32} \mathrm{P}\right]$ ATP alone were applied as internal controls. Samples were incubated with $\gamma-\left[{ }^{32} \mathrm{P}\right]$ ATP and proteins were separated by SDS-PAGE. Phosphorylated bands were visualized by a phosphorimager. A, lane 1-6 represent indicated amounts of purified MBP-PIP5K2. Black arrowheads point at protein sizes, the white arrowhead indicates the size of the MBP-PIP5K2 fusion protein. $B$, the extent of $P^{32}$ incorporation was quantified and is expressed as photo stimulated luminescence (PSL). Black bars indicate the extent of MBP-PIP5K2 phosphorylation after incubation with LE, grey bars represent $P^{32}$ incorporation without LE. n.d., not detected. Data are replicates of two independent experiments and show similar results. 
Next to phosphorylated MBP-PIP5K2 at $120 \mathrm{kDa}$, two additional phosphorylated bands at around $65 \mathrm{kDa}$ and $40 \mathrm{kDa}$ were detected in lanes 1, 3 and 5, which were also present in the leaf extract control (lane 6) and thus, represent intrinsic components of the leaf extract. When $5 \mu \mathrm{g}$ or $10 \mu \mathrm{g}$ of recombinant MBP-PIP5K2 were used a low degree of autophosphorylation of MBP-PIP5K2 was detectable (Fig. 3.4 A lane 2, 4 and 6). As another negative control, denaturated leaf extract alone was also tested for its ability to get phosphorylated but no phosphorylated bands were detectable (Fig. 3.4).

To verify the identity of the recombinant MBP-PIP5K2 protein and to test if the N-terminal MBP-tag contains phosphorylation motifs that can be recognized by an endogenous plant protein kinase and therefore lead to the detected phosphorylation of the MBP-PIP5K2 fusion protein, leaf extract-treated MBP-PIP5K2 was subjected to restriction by a TEV protease as has been described in chapter 3.2.1. A section of the Coomassie-stained SDS-PAGE gel and the corresponding autoradiograph is shown in Figure 3.5 A and B, respectively.

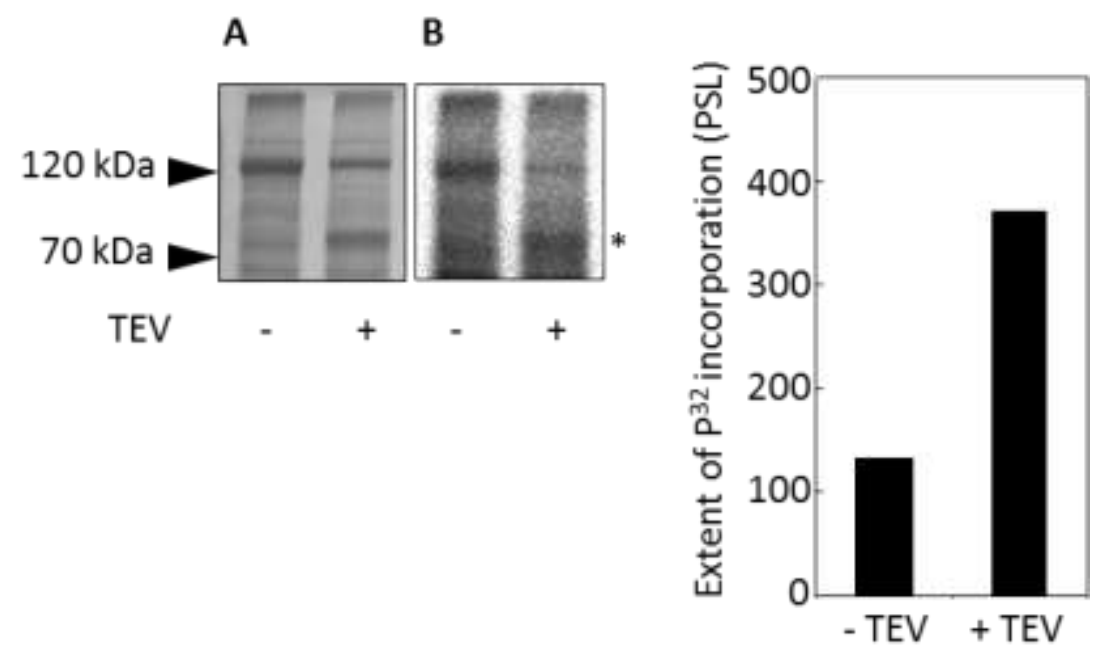

Fig. 3.5: TEV-treatment of phosphorylated MBP-PIP5K2 indicates phosphorylation of PIP5K2. Recombinantly expressed and purified MBP-PIP5K2 was treated with Arabidopsis leaf extract and TEV protease as indicated (+/-). Samples were separated by SDS-PAGE, Coomassie-stained (A) and a phosphorimage was exposed (B). Phosphorylation intensities at the $70 \mathrm{kDa}$ band $\left({ }^{*}\right)$ were quantified and illustrated in the graphic (right). Data were repeated twice and showed comparable results.

The phosphorylated MBP-PIP5K2 full-length protein investigated at $120 \mathrm{kDa}$ and was clearly detectable in the Coomassie-stained SDS-PAGE gel and in the phosphorimage (Fig. 3.5 B lane 1). After TEV-treatment, a smaller band was of approx. $70 \mathrm{kDa}$ in size and although a band with this size appeared in the Coomassie-stained gel (Fig. $3.5 \mathrm{~A}$ lane 2), a phosphorylated full-length band could still be identified at $120 \mathrm{kDa}$ in the phosphorimage (Fig. $3.5 \mathrm{~B}$ lane 2), suggesting that the MBP-PIP5K2 protein was not completely cleaved into MBP and PIP5K2 fragments. The band at the same position in the phosphorimage was slightly indistinct (Fig. 3.5 B lane 2). Therefore radiolabeled signals at $70 \mathrm{kDa}$ (Fig. $3.5 \mathrm{~B}$, asterisk) were quantified and compared between TEV-treated (+ TEV) and untreated (- TEV) samples as illustrated in Figure 3.5. After 
TEV-treatment the level of phosphorylation at $70 \mathrm{kDa}$ increased compared to untreated samples, suggesting that the appearing $70 \mathrm{kDa}$ PIP5K2 band is indeed phosphorylated.

The data imply that Arabidopsis PIP5K2 can not only be phosphorylated in vitro by an artificial PKA but is also phosphorylated by one or more protein kinases present in an endogenous plant extract. The next step towards understanding the functionality of this phosphorylation in the regulation of PIP5K2 was to test for changes in the in vitro activity after incubating recombinant PIP5K2 with PKA or plant extracts, respectively.

\subsection{In vitro phosphorylation of recombinant PIP5K2 reduces catalytic activity}

The influence of incubating PIP5K2 with leaf extract on PI4P 5-kinase activity was analysed by incubating purified MBP-PIP5K2 with PKA and $5 \mathrm{mM}$ of cold ATP. Phosphorylated MBP-PIP5K2 was then tested for activity with PtdIns4P and radiolabeled $y-\left[{ }^{32} P\right]$ ATP as substrates and the amount of synthesized Ptdlns $(4,5) \mathrm{P}_{2}$ was quantified. Equal amounts of purified MBP-PIP5K2 incubated with $5 \mathrm{mM}$ of cold ATP and buffer instead of PKA served as control and was analysed under the same conditions. Figure 3.6 illustrates PI4P 5-kinase activities observed expressed as percent activity compared to the buffer-treated MBP-PIP5K2. The results indicate that the activity of MBP-PIP5K2 decreased significantly about $50 \%$ after treatment with PKA and cold ATP compared to the untreated control. PKA alone had no PI4P 5-kinase activity. To test whether PI4P 5-kinase activity can be rescued by dephosphorylation of MBP-PIP5K2, SAP was applied but showed no difference in catalytic activity to PKA treated MBP-PIP5K2 (data not shown).

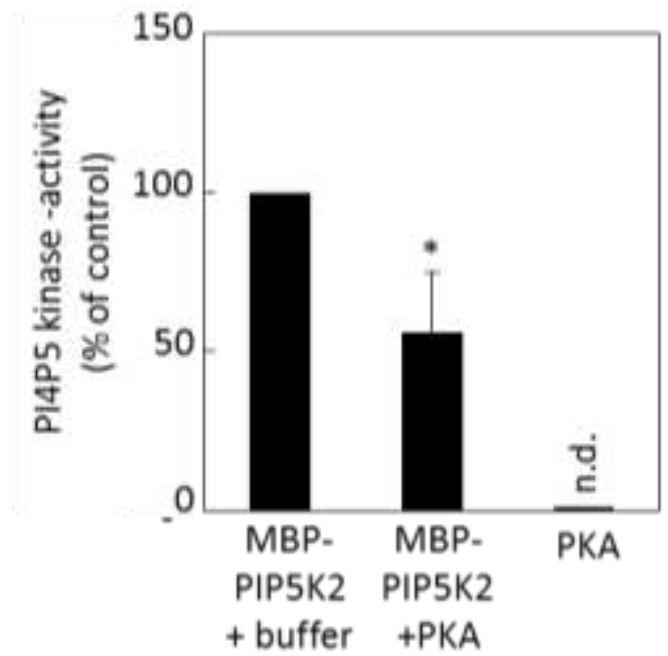

Fig. 3.6: PIP5K2 is inhibited by PKA. Purified MBP-PIP5K2 was incubated with buffer or PKA followed by activity tests with radiolabled $\mathrm{Y}-\left[{ }^{32} \mathrm{P}\right]$ ATP and Ptdlns4P as substrates. Lipid products were extracted, separated by TLC and the extent of synthesized PtdIns $(4,5) \mathrm{P}_{2}$ was quantified by phosphoimaging. Data represent the means \pm SD of three independent experiments, each assayed in duplicates. The asterisk indicates a significant decrease of PI4P 5-kinase activity after incubation with PKA compared to the control sample according to a student's t-test $(p=0.015)$. n.d, not detected. 
Although the phosphorylation of PIP5K2 by a mammalian PKA must be seen as an artificial modification, the decrease in activity after PKA-treatment gave first hints towards the function of such a modification.

To verify whether phosphorylation by an endogenous plant protein kinase had similar effects on activity, purified MBP-PIP5K2 was treated with leaf extract of 6-week-old plants. To furthermore test whether the effect observed depended on the availability of ATP as a protein kinase co-substrate, different concentrations of cold ATP were applied. The ability to form Ptdlns $(4,5) \mathrm{P}_{2}$ was tested as described for the PKA-treatment and the percent activity of each sample was compared to a control incubated with $5 \mathrm{mM}$ ATP and buffer instead of leaf extract as shown in Figure 3.7. Incubation with leaf extract and $5 \mathrm{mM}$ of cold ATP significantly decreased the PI4P 5-kinase activity by about $60 \%$. A treatment without cold ATP or with only $1 \mathrm{mM}$ ATP in the presence of leaf extract did not alter PI4P 5-kinase activity of the recombinant enzyme (Fig. 3.7 A). Leaf extract alone had no detectable PI4P 5 -kinase activity.

A

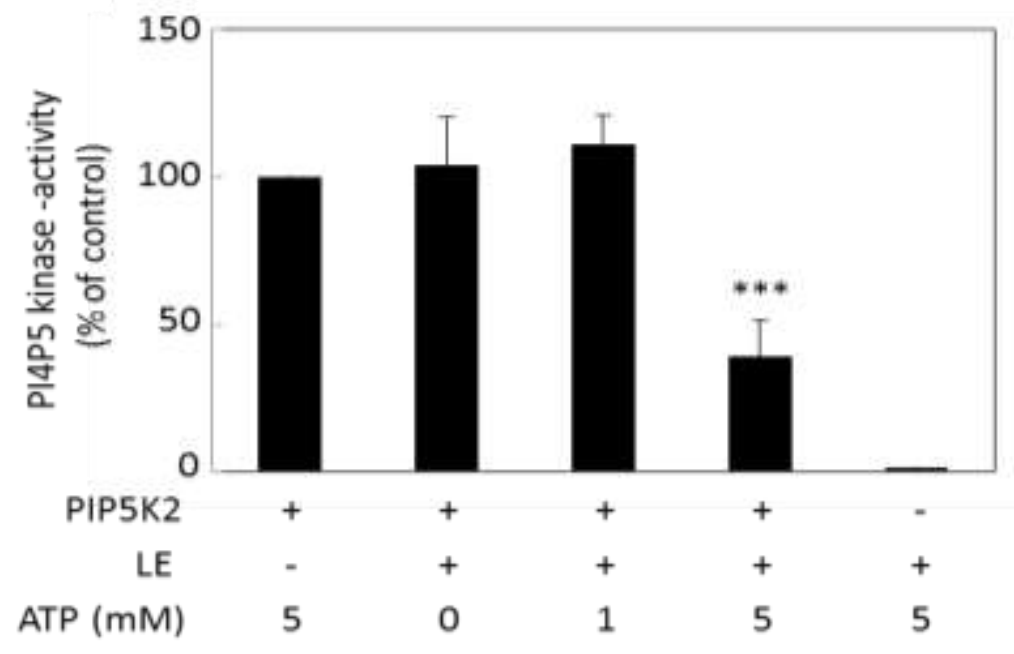

B

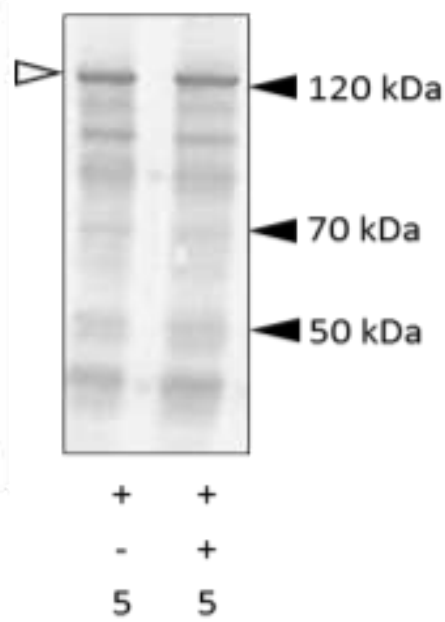

Fig. 3.7: MBP-PIP5K2 activity is inhibited by leaf extract in an ATP-dependent fashion not related to protein degradation. Equal amounts of purified MBP-PIP5K2 were incubated with leaf extract (LE) and 0-5 mM of cold ATP. The activity was tested by applying radiolabled $v-\left[{ }^{32} \mathrm{P}\right]$ ATP and Ptdlns4P. Lipid products were extracted, analyzed by TLC and quantified by phosphoimaging. A, PI4P 5 kinase activity of each sample in percent compared to a control incubated with buffer and $5 \mathrm{mM}$ of cold ATP only (left). Data represent the means \pm SD of 3-5 independent experiments, each assayed in duplicates. The asterisks indicate a significant decrease of PI4P 5-kinase activity after incubation with LE and 5 mM ATP compared to the buffer-treated control according to a student's t-test $(p<0.001)$. B, Samples incubated with and without LE and $5 \mathrm{mM}$ cold ATP were analyzed by immunoblot. White arrowheads represent the size of the MBP-PIP5K2 fusion protein. Black arrowheads show protein sizes. ). Immunoblot analyses were repeated twice and showed comparable results.

To verify that the observed decrease in PI4P 5-kinase activity after incubation of MBP-PIP5K2 in leaf extract and $5 \mathrm{mM}$ of cold ATP was not induced by degradation of the fusion protein an immunoblot was performed as depicted in Figure 3.7 B. Equal protein concentrations of MBPPIP5K2 were incubated with $5 \mathrm{mM}$ of cold ATP in the presence or in absence of leaf extract. 
Samples were separated by SDS-PAGE and developed using an MBP antibody. A band at 120 kDa was detected as the MBP-PIP5K2 full-length protein (white arrowhead) and is clearly detectable in both samples independent from their treatment with leaf extract. Therefore, the reduced activity observed upon treatment of recombinant MBP-PIP5K2 with leaf extract was not a consequence of degradation of the recombinant protein.

\subsection{Determination of possible phosphorylation sites in PIP5K2}

The data shown in the previous section imply that phosphorylation of PIP5K2 contributes to its regulation. To further analyse the role of phosphorylation by biochemical means it is necessary to identify possible phosphorylation sites based on the PIP5K2 sequence. The $70 \mathrm{kDa}$ protein PIP5K2 consists of 754 amino acids and contains at least five recognizable domains: a short $\mathrm{N}$ terminal (NT) domain, a membrane-occupation-and-recognition-nexus (MORN-) domain, a linker (Lin) domain, a dimerization (Dim) domain and catalytic (Cat) domain (Fig. 3.8). The Nterminal domains distinguish the plant PI4P 5-kinases from the animal PIP-kinases and have putative regulatory roles. As already described in the introduction, the MORN-domain is thought to function in plasma membrane binding (Takeshima et al., 2000; Im et al., 2007) and it was shown previously that the Lin-domain may also have important functions in subcellular localization (Stenzel et al., 2012).



Cat

Fig. 3.8: Arabidopsis PIP5K2 consists of several domains. NT, N-terminal domain; MORN, membraneoccupation-and-recognition-nexus domain; Lin, linker domain; Dim, dimerization domain; Cat, catalytic domain.

In the proposed model (Fig. 1.6, c.f. section 1.6) it is hypothesized that phosphorylation regulates PI4P 5-kinase activity by inhibiting proper binding to the PI substrate and/or correct localization of the enzyme to its target membrane. Since the $\mathrm{N}$-terminal regions have a supposed function in localizing PIP-kinases to the plasma membrane it was first tested whether successive $\mathrm{N}$-terminal deletions have effects on the phosphorylation status of MBPPIP5K2. 


\subsubsection{A systematic deletion approach to delineate PIP5K2 protein regions important for phosphorylation effects on activity}

The N-terminal domain of PIP5K2 is about 429 amino acids long and contains 32 serine-, 22 threonine- and 15 tyrosine-residues. The sequence is illustrated in Figure 3.9. To investigate whether the N-terminal regions exert an influence on phosphorylation of PIP5K2, a number of truncations were made, the altered proteins recombinantly expressed in E. coli and analyzed for their patterns of phosphorylation and in vitro activity after incubation with Arabidopsis leaf extract.

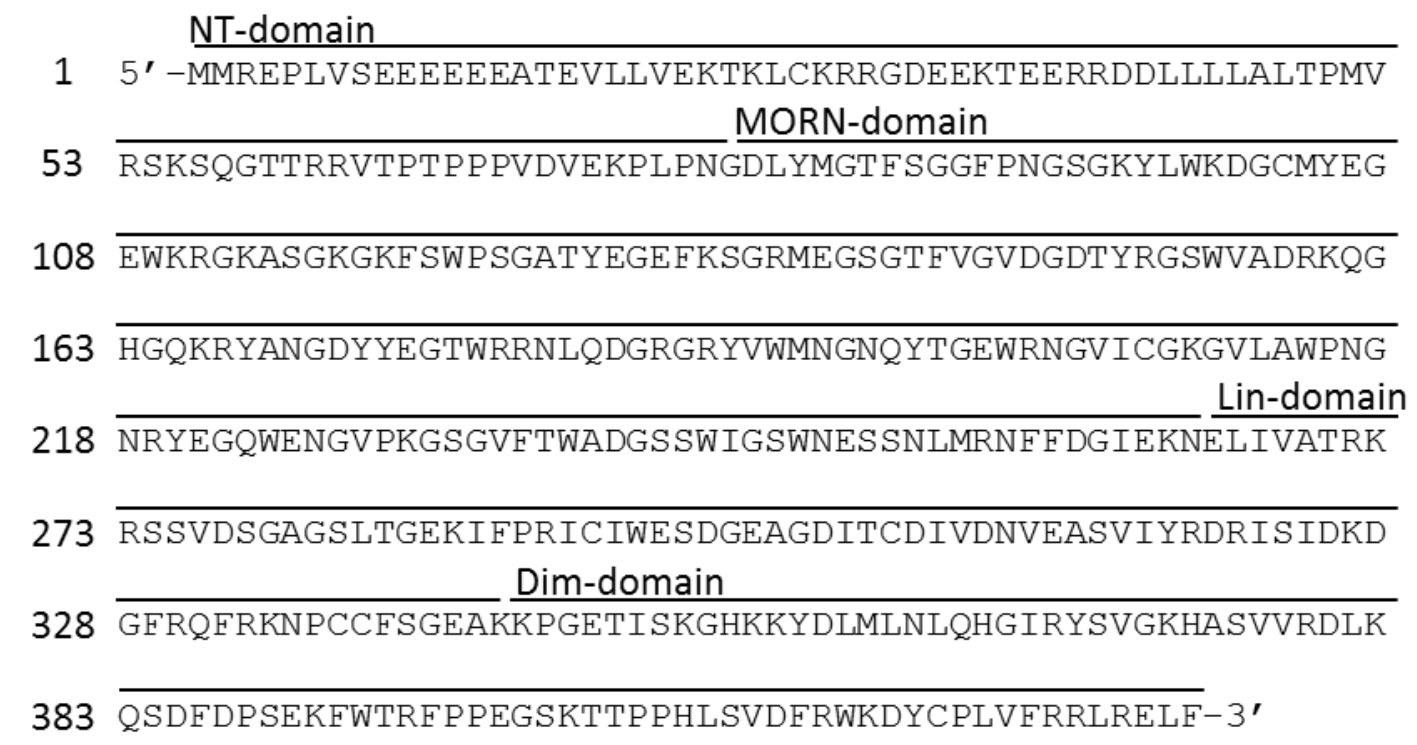

Fig. 3.9: Sequence of the $\mathrm{N}$-terminal domain of PIP5K2. The domains are assigned to the corresponding sequences as indicated. NT, N-terminal domain; MORN, membrane-occupation-and-recognition-nexus domain; Lin, linker domain; Dim, dimerization domain; Cat, catalytic domain.

\subsubsection{Phosphorylation of PIP5K2 truncations by Arabidopsis leaf extract}

Three PIP5K2-truncations were created by the systematic deletion of $\mathrm{N}$-terminal domains. The truncated variants of PIP5K2 are defined as follows: $\Delta$ NT-MORN, PIP5K2 260-754; $\Delta$ NTMORN-Lin, PIP5K2 344-754; and the catalytic domain alone, PIP5K2 429-754. The truncations are illustrated in Figure 3.10 A. The truncations were expressed as fusions to an $\mathrm{N}$-terminal MBP-tag in E. coli Rosetta 2 cells as described before (section 2.10.4) and purified via an MBP trap matrix. The constructs were kindly provided by Dr. Irene Stenzel (MLU Halle-Wittenberg). To verify enrichment, crude extracts, wash fractions and eluted fractions of full-length MBPPIP5K2, MBP- $\triangle$ NT-MORN, MBP- $\triangle$ NT-MORN-Lin and MBP-catalytic domain were separated by SDS-PAGE and stained with Coomassie (see appendix Fig. 7.1). Figure 3.10 B illustrates the separated elution fractions of full-length PIP5K2 and the truncated versions. Major bands were 
detectable at a size of $120 \mathrm{kDa}$ (full-length MBP-PIP5K2, lane 1), $100 \mathrm{kDa}$ (MBP- $\triangle$ NT-MORN, lane 2), $90 \mathrm{kDa}$ (MBP- $\triangle \mathrm{NT}-\mathrm{MORN}$-lin, lane 3) and $80 \mathrm{kDa}$ (MBP-catalytic domain, lane 4) that correlated to the expected size of the applied truncations.

To test for phosphorylation by an endogenous Arabidopsis protein kinase, equal amounts of purified full-length MBP-PIP5K2 and corresponding truncations were incubated with plant extract of 6-weeks-old leaves and radiolabeled $y-\left[{ }^{32} \mathrm{P}\right]$ ATP (see section 2.11 .1 and 3.2.2). Proteins were separated by SDS-PAGE and radiolabeled proteins were quantified by phosphorimaging. The extent of phosphorylation of truncated MBP-PIP5K2 variants was compared to phosphorylation of full-length MBP-PIP5K2 and is illustrated in figure $3.10 \mathrm{C}$. Deletion of the extreme $\mathrm{N}$-terminal domain and the MORN-domain decreased the phosphorylation status compared to wild type MBP-PIP5K2 by about $30 \%$ (Fig. $3.10 \mathrm{C}$ lane 2). The additional removal of the Lin-domain increased the extent of $\mathrm{P}^{32}$ incorporation again to wild type level (Fig. $3.10 \mathrm{C}$ lane 3). Recombinant MBP-Cat-domain displayed ca $50 \%$ phosphorylation compared to the full-length enzyme.

A

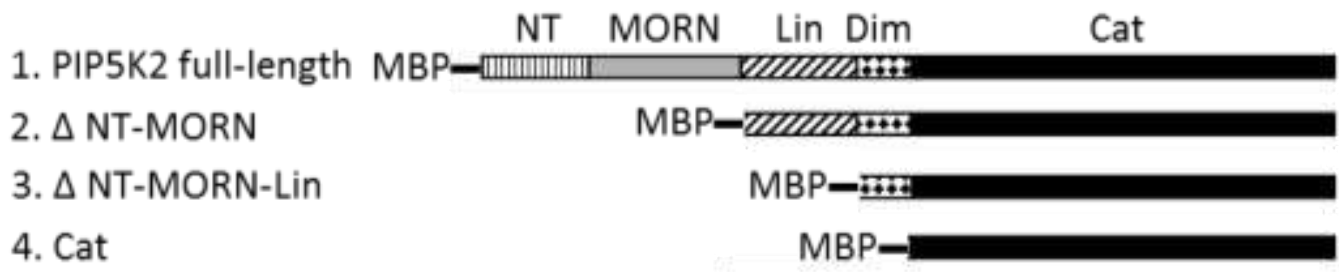

B

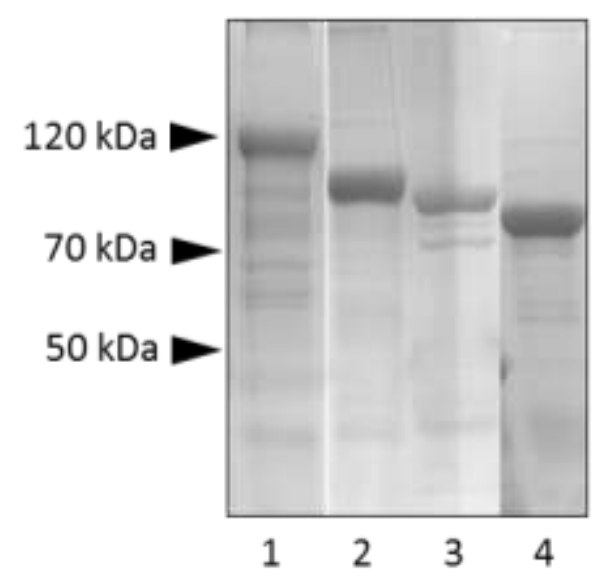

C



Fig. 3.10: Phosphorylation of truncated PIP5K2 variants by Arabdiopsis leaf extract. A, Truncated MBPPIP5K2 versions were created. NT, N-terminal domain; MORN, membrane-occupation-and-recognitionnexus domain; Lin, linker domain; Dim, dimerization domain; Cat, catalytic domain. B, Coomassie stained SDS-PAGE of purified full-length PIP5K2 and corresponding truncations. Purified proteins were subjected to phosphorylation by an Arabidopsis leaf extract and radiolabeled $\gamma-\left[{ }^{32} \mathrm{P}\right]$ ATP. Proteins were separated by SDS-PAGE and phosphorylated proteins were visualized by phosphorimaging. The extent of phosphorylation was measured and compared to the phosphorylation of full-length MBP-PIP5K2 (C). Data represent means \pm SD of 2-3 independent experiments. 


\subsubsection{Effects of an Arabidopsis plant extract on catalytic activity of PIP5K2 truncations}

In the last section the influence of an Arabidopsis plant extract on the phosphorylation status of the PIP5K2 truncations was investigated. During these experiments it was observed that the deletion of the extreme $\mathrm{N}$ terminal domains exceeded effects on the phosphorylation status of the PIP5K2 protein. In the next section it was therefore analyzed whether incubation with Arabidopsis plant extract also affected the activity of the respective PIP5K2 truncations.

To test for changes in PI4P 5-kinase activity purified MBP-PIP5K2 and the corresponding truncations were incubated with plant extract of 6-week-old leaves and $5 \mathrm{mM}$ of cold ATP. The corresponding buffer-treated samples were used as controls. After incubation, lipid kinase activity was tested by supplying radiolabeled $\mathrm{Y}-\left[{ }^{32} \mathrm{P}\right]$ ATP and Ptdlns4P. Product lipids were extracted, separated by TLC and the amount of synthesized Ptdlns $(4,5) \mathrm{P}_{2}$ was quantified by phosphoimaging. Figure 3.11 illustrates the relative activity of the recombinant proteins after incubation with leaf extract by comparing the amount of produced $\operatorname{Ptdlns}(4,5) \mathrm{P}_{2}$ of leaf extract-treated samples to that of the respective buffer-treated controls. As described in section 3.3, incubation of full-length MBP-PIP5K2 with leaf extract increased activity about $70 \%$ compared to untreated MBP-PIP5K2 (cf. Fig. 3.7). The activity of MBP- $\triangle$ NTT-MORN was only slightly reduced after incubation with LE compared to the untreated MBP- $\triangle N T-M O R N$ control. Similar results were observed when MBP- $\triangle$ NT-MORN-Lin and catalytic domain alone were subjected to leaf extract-treatment.

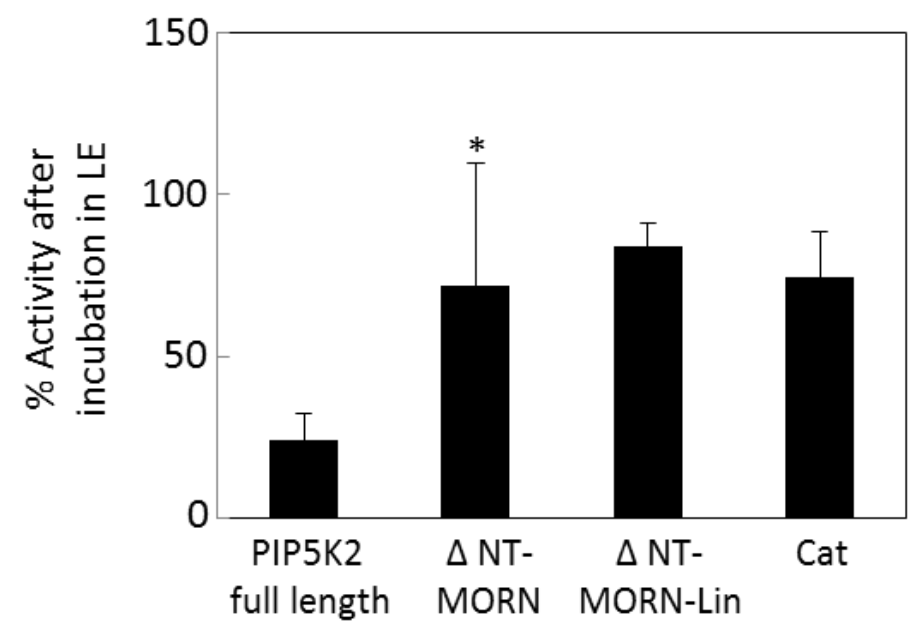

Fig. 3.11: Influence of Arabidopsis leaf extract on activity of MBP-PIP5K2 and truncated versions. Purified MBP-PIP5K2 and MBP-PIP5K2 truncations were incubated with leaf extract and $5 \mathrm{mM}$ of ATP following radiolabeled activity tests. Ptdlns $(4 ; 5) \mathrm{P}_{2}$ synthesized by treated samples was compared to that of the corresponding untreated samples and the respective individual proportions for each sample pair were expressed as \% activity. Data are means \pm SD of 3-4 experiments. The asterisk indicates a significant difference between PI4P 5-kinase activity of LE-treated wild type control and LE-treated MBP$\triangle$ NT-MORN according to a student's t-test $(p<0.001)$ 
In summary, MBP-PIP5K2 truncations were only slightly influenced by leaf extract-treatment compared to wild type PIPK52 (Fig. 3.11), suggesting that NT- and/or MORN- domains contain residues that are targets for phosphorylation with effects on catalytic activity.

\subsubsection{Identification of phosphorylation sites using databases}

The results observed in the previous section indicated that the NT- and MORN-domain might hold possible phosphorylation sites, important for regulating PIP5K2-activity. To biochemically analyse the effects of phosphorylation on catalytic activity and membrane localization it is important to determine phosphorylation with confidence. The first step in the determination of possible phosphorylation sites was the review of information in databases that are freely available on the internet. One of the databases used is the Arabidopsis Protein Phosphorylation Site Database (PhosPhAt 4.0) (Heazlewood et al., 2008) which provides a portal for information on Arabidopsis phosphorylation sites identified by mass spectrometry in large scale experiments made available by different research groups. Review of the database information available for different Arabidopsis PI4P 5-kinase isoforms revealed that several isoforms were phosphorylated. The database revealed a phosphorylation site for PIP5K2 at position S409 (Engelsberger et al., 2012). Because of the substantial similarities between PI4P 5-kinase isoforms of Arabidopsis, PhosPhAT 4.0 was also searched for phosphorylation sites identified in other PI4P 5-kinase isoforms. A phosphorylation site was determined for Arabidopsis PIP5K3 at position T472 (Engelsberger et al., 2012), a residue conserved in PIP5K2 at position T500 (Fig. 3.12).

Besides the review of experimental data deposited in databases, in a second approach the sequence of PIP5K2 was evaluated with the NetPhos prediction software (Blom et al., 1999). Since protein phosphorylation can only occur on amino acids with hydroxyl group (serine, threonine and sometimes tyrosine) NetPhos predicts phosphorylation on those three residues in eukaryotic proteins. The prediction is based on an algorithm and the service is freely available on the website http://www.cbs.dtu.dk/services/NetPhos/. The residues showing a high prediction score and conservation within the Arabidopsis PI4P 5-kinase family (Fig. 3.12) were chosen for further investigations and are listed in Table 3.2. 


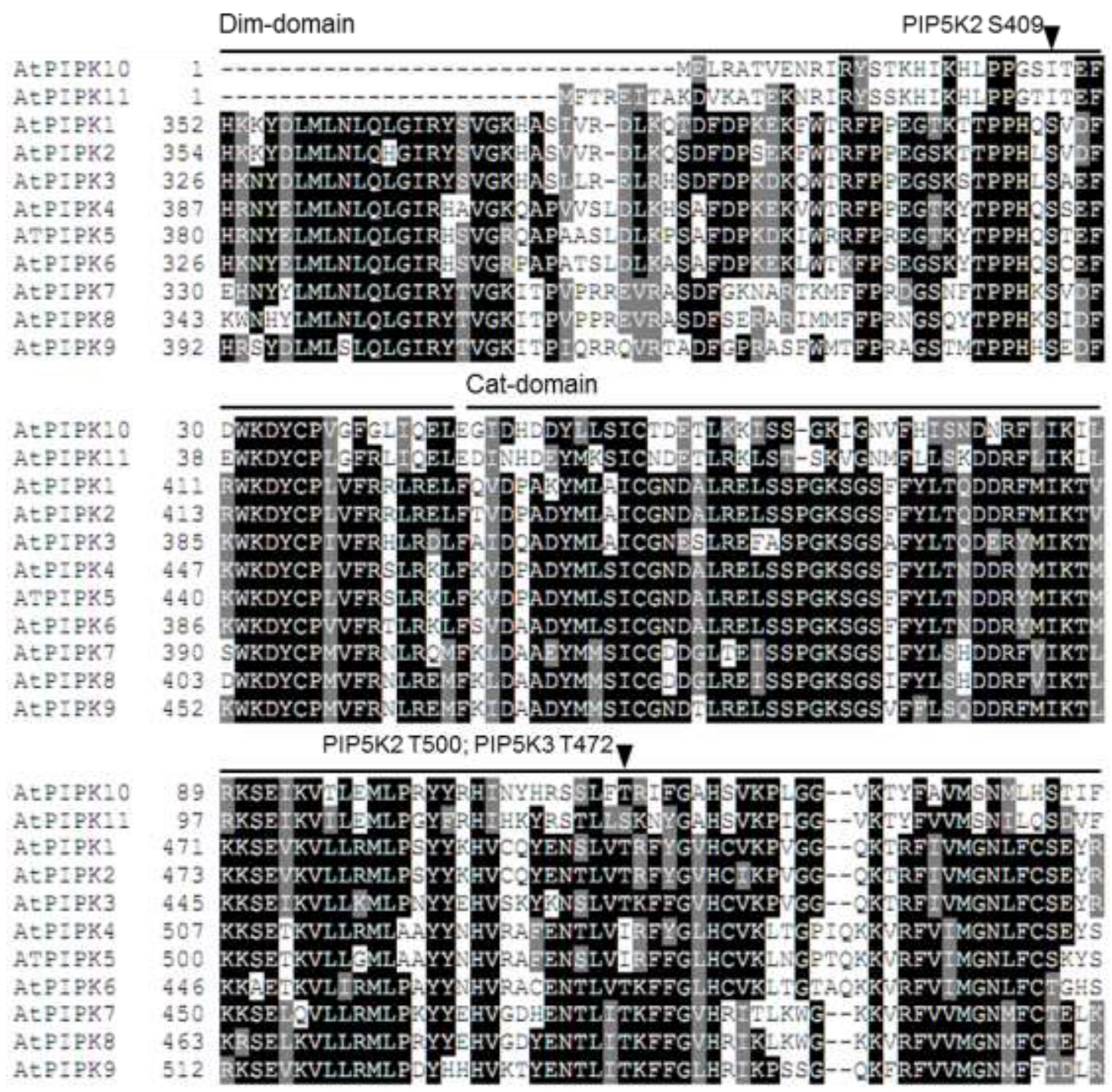

Fig. 3.12: Arabidopsis PI4P 5-kinase isoforms display high sequence similarity. Local alignment of part of the catalytic portions of the 11 Arabidopsis PI4P 5-kinase isoforms. Black areas represent identical amino acids. Grey areas show similar amino acids. Dim, dimerization domain; Cat, catalytic domain.

Table 3.2: Prediction of possible phosphorylation sites in Arabidopsis PIP5K2. The prediction of putative phosphorylation sites in PIP5K2 was evaluated by the NetPhos prediction software. Serine, threonine and tyrosine residues were rated with numbers between zero and one describing a low or a high chance of phosphorylation, respectively. Amino acids were considered as "conserved" when found in 5 or more isoforms.
Amino acid position
Prediction score
conserved

\begin{tabular}{ccc}
\hline S370 & 0.37 & Yes \\
S475 & 0.63 & Yes \\
S541 & 0.94 & Yes \\
T546 & 0.99 & yes \\
\hline
\end{tabular}




\subsubsection{Determination of phosphorylation sites by MS}

Although the research on PhosPhAT 4.0 revealed several phosphorylated residues in PIP5K2 and PIP5K3 it has to be noticed that these sites were identified in large scale experiments with cell cultures exposed to nitrogen starvation. In the context of this work it was important to identify phosphorylation sites in PIP5K2 after treatment with an Arabidopsis leaf extract. Therefore, an experimental setup was chosen to determine additional phosphorylation sites by mass spectrometry. MBP-PIP5K2 was treated with leaf extract, digested with trypsin (see section 2.11) and the digested peptides were subjected to MS analysis by Dr. Waltraud Schulze (MPI, Golm). Phosphorylated peptides were identified by comparing the identified peptide masses with the calculated peptide masses of trypsin digested PIP5K2. A mass difference of $96 \mathrm{Da}$ between two tryptic peptide fragments of corresponding sequences indicates phosphorylation. The analysed peptides covered over $80 \%$ of the PIP5K2 sequence as shown in Figure 3.13. New Phosphorylation sites were identified at position T430 and S598. The corresponding spectra are attached in the appendix (Fig. 7.2).

$\begin{array}{rlllll}1 & \text { MMREPLVSEE } & \text { EEEEATEVLL } & \text { VEKTKLCKRR } & \text { GDEEKTEERR } & \text { DDLLLLALTP } \\ 51 & \text { MVRSKSQGTT } & \text { RRVTPTPPPV } & \text { DVEKPLPNGD } & \text { LYMGTFSGGF } & \text { PNGSGKYLWK } \\ 101 & \text { DGCMYEGEWK } & \text { RGKASGKGKF } & \text { SWPSGATYEG } & \text { EFKSGRMEGS } & \text { GTFVGVDGDT } \\ 151 & \text { YRGSWVADRK } & \text { QGHGQKRYAN } & \text { GDYYEGTWRR } & \text { NLQDGRGRYV } & \text { WMNGNQYTGE } \\ 201 & \text { WRNGVICGKG } & \text { VLAWPNGNRY } & \text { EGQWENGVPK } & \text { GSGVFTWADG } & \text { SSWIGSWNES } \\ 251 & \text { SNLMRNEFG } & \text { IEKNELIVAT } & \text { RKRSSVDSGA } & \text { GSLTGEIFP } & \text { RICIWESDGE } \\ 301 & \text { AGDITCDIVD } & \text { NVEASVIYRD } & \text { RISIDKDGFR } & \text { QFRKNPCCFS } & \text { GEAKKPGETI } \\ 351 & \text { SKGHKKYDLM } & \text { LNLQHGIRYS } & \text { VGKHASVVRD } & \text { LKQSDFDPSE } & \text { KFWTRFPPEG } \\ 401 & \text { SKTTPPHLSV } & \text { DFRWDYCPL } & \text { VFRRLRELFT } & \text { VDPADYMLAI } & \text { CGNDALRELS } \\ 451 & \text { SPGKSGSFFY } & \text { LTQDDRFMIK } & \text { TVKKSEVKVL } & \text { LRMLPSYYKH } & \text { VCQYENTLVT } \\ 501 & \text { RFYGVHCIKP } & \text { VGGQKTRFIV } & \text { MGNLFCSEYR } & \text { IQRRFDLKGS } & \text { SHGRYTSKPE } \\ 541 & \text { GEIDETTTLK } & \text { DLDLNEAFRI } & \text { QRNWYQELT } & \text { QIKRDCEFLE } & \text { AERIMDYSLL } \\ 601 & \text { VGVHFRDDNT } & \text { GDKMGLSPFV } & \text { LRSGKIESYQ } & \text { SEKFMRGCRF } & \text { LEAELQDMDR } \\ 651 & \text { ILAGRKPLIR } & \text { LGANMPARAE } & \text { RMARRSDYDQ } & \text { YSSGGTNYQS } & \text { HGEVYEVVIY } \\ 701 & \text { FGIIDILQDY } & \text { DISKKIEHAY } & \text { KSLQADPASI } & \text { SAVDPKLYSR } & \text { RFRDFISRIF } \\ 751 & \text { IEDG } & & & & \end{array}$

Fig. 3.13: Sequence coverage of PIP5K2 after MS-analysis of trypsin digested peptides. Purified recombinant MBP-PIP5K2 was prephosphorylated in vitro, digested with trypsin and the resulting peptides were analysed by mass spectrometry. Shown is the amino acid sequence of PIP5K2. Black, identified peptides. Grey, not accessible to MS. Underlined, determined phosphorylation sites.

\subsubsection{Identification of phosphorylation sites by Peptide Array analysis}

Although two new phosphorylation sites of PIP5K2 were experimentally determined at position T430 and S598 via mass spectrometry, it is possible that Arabidopsis PIP5K2 is phosphorylated at yet more positions. To identify additional phosphorylation sites in PIP5K2, and to verify phosphorylation sites already determined by MS-analyses and computer aided 
prediction, PepStar ${ }^{\mathrm{TM}}$ peptide arrays (JPT Peptide Technologies GmbH, Berlin, Germany) were used as described in section 2.11.3.

Peptide Arrays were obtained from JPT Peptide Technologies $\mathrm{GmbH}$ and represent around 250 15-mer peptides (in triplicates) based on the PIP5K2 amino acid sequence (for details see section 2.11.3). In a first round of experiments, peptide arrays were incubated with PKA and $y$ $\left[{ }^{32} \mathrm{P}\right]$ ATP. Radiolabeled peptides were visualized by exposing a phosphorimager screen for 1 day (Fig. 3.14). Dark signals on the phosphorimage represent radiolabeled peptides and were detected in each of the three subarrays at identical positions (Fig. 3.14 A). Figure 3.14 B depicts an enlarged subarray. The corresponding peptide map was supplied with the kit allowing the assignment of each spot on the peptide array to its peptide sequence. Overall 16 radiolabeled peptide sequences were identified by the peptide array (Fig. 3.14 B, black arrowheads) and are illustrated in the appendix in Figure 7.3. Based on the peptide sequence the following PIP5K2 residues were identified as sites phosphorylated by PKA: T270, S274, S275, S323, S370, S409; S455, S456, T462, S676 and S739.

A

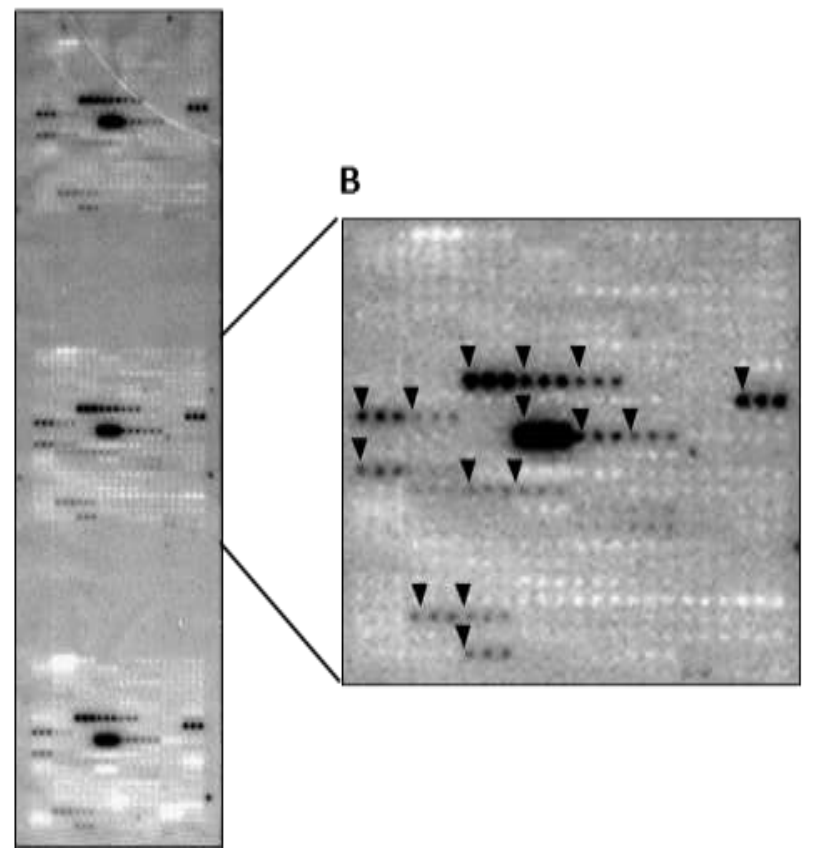

C



Fig. 3.14: PKA phosphorylates several PIP5K2 peptides. Based on the PIP5K2 sequence overlapping 15mer peptides were synthesised and spotted in triplicates onto a surface matrix. The peptide array is printed in three identical subarrays. A, phosphorimage of a peptide array after incubation with PKA and radiolabeled $\gamma-\left[{ }^{32} \mathrm{P}\right]$ ATP. B, Enlarged subarray of the same peptide array. The phosphorimage was exposed overnight. Each spot is defined by one peptide and can be identified by a peptide map supplied with the kit. Black arrowheads indicate radiolabeled peptides. C, Phosphorimage of a peptide array after incubation with Arabidopsis leaf extract. The phosphorimage was exposed for 7 days.

After the successful phosphorylation of the PIP5K2 peptide array by PKA the phosphorylation treatment was also attempted with Arabidopsis leaf extract (Fig. 3.14 C). In order to identify 
peptides that are phosphorylated by an endogenous plant protein kinase, peptide arrays were incubated with $150 \mu \mathrm{g}$ of protein from freshly prepared Arabidopsis leaf extract and radiolabeled $\psi-\left[{ }^{32} \mathrm{P}\right]$ ATP. The incubation was carried out as described for the treatment with PKA (see section 2.11.3). Figure 3.14 C depicts a phosphorimage of a peptide array incubated with $150 \mu \mathrm{g}$ protein extract. Although the peptide array was exposed for 7 days no radiolabeled peptides were detectable on the corresponding phosphorimage (Fig. 3.14 C). In a next experiment the amount of applied plant protein was increased to $300 \mu \mathrm{g}$ and $800 \mu \mathrm{g}$, respectively, but still no radiolabeled peptides could be identified (not shown).

Although the peptide array was not successful for an application with leaf extract the combined investigations so far have identified several candidate amino acid positions of PIP5K2 that might function as phosphorylation sites and are summarized in Table 3.3. The detected candidate amino acids are mostly localized in the catalytic domain (T430, S475, T500, S541, T546 and S598). Only two putative phosphorylation sites are located in the N-terminal domains (S370 and S409). For better overview, the phosphorylation sites further investigated are illustrated on a removable map is supplied in the appendix of this thesis (Fig. 7.4).

Table 3.3: Summary of predicted and experimentally verified phosphorylation sites chosen for further investigations. The amino acid positions listed are potential phosphorylation sites and were determined by computer aided prediction and mass spectrometry. Lin, linker domain; Dim, dimerization domain; Cat, catalytic domain.

\begin{tabular}{|c|c|c|c|}
\hline & Amino acid position & Domain & Identified with \\
\hline \multirow{4}{*}{ 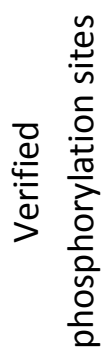 } & S409 & Dim & Mass spectrometry (PhosPhAT 4.0) \\
\hline & T430 & Cat & Mass spectrometry (this thesis) \\
\hline & T500 & Cat & $\begin{array}{l}\text { Homologous to PIP5K3 T472; defined by } \\
\text { mass spectometry (Phosphat 4.0) }\end{array}$ \\
\hline & S598 & Cat & Mass spectrometry (this thesis) \\
\hline \multirow{4}{*}{ 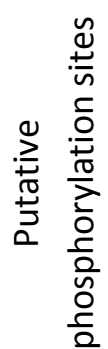 } & S370 & $\operatorname{Lin}$ & Computer aided prediction \\
\hline & S475 & Cat & Computer aided prediction \\
\hline & S541 & Cat & Computer aided prediction \\
\hline & T546 & Cat & Computer aided prediction \\
\hline
\end{tabular}




\subsection{Site-directed mutagenesis of selected serine and threonine residues in PIP5K2}

The sites found by MS and by computer aided prediction (Table 3.3) were chosen for further characterization of the effects of possible phosphorylation on the function of PIP5K2. As outlined in the material and methods section, selected serine or threonine residues of PIP5K2 were altered to $A$, resembling a dephosphorylated state (phosphoablation), or to $D$, mimicking constitutive phosphorylation (phosphomimicry). The resulting A/D variants of PIP5K2 were recombinantly expressed in $E$. coli and subjected to biochemical characterization.

\subsection{In vitro activity of recombinantly expressed PIP5K2 variants}

Based on the model described in the introduction, it was the working hypothesis of this study that the A-variant of a specific phosphorylation site should imitate dephosphorylated PIP5K2 and be catalytically active. Reciprocally, the D-variant mimicking the phosphorylated state of the enzyme should be less active than the A-variant or the respective wild type enzyme. To analyse whether the substitution of predicted and verified phosphorylation sites displayed effects as hypothesised, the amino acid positions S370, S409, S475, T430, T500, S541, T546 and S598 were all individually altered by site directed mutagenesis to A or D. The corresponding PIP5K2 A/D-variants were then expressed in E. coli Rosetta 2 cells as described in section 2.10.4. Crude extracts were analysed for catalytic activity by applying Ptdlns4P and radiolabeled $\gamma-\left[{ }^{32} \mathrm{P}\right]$ ATP for $1 \mathrm{~h}$. Lipid products were extracted, separated by TLC and Ptdlns $(4,5) \mathrm{P}_{2}$ synthesized was quantified by phosphorimaging.

The characterization of $A / D$-variants that were mutated based on experimentally verified phosphorylation sites is depicted in Figure 3.15. The activity of equal protein amounts of each variant was compared to the catalytically active PIP5K2 wild type control (Fig. 3.15 white bar) and is illustrated as percent activity of the control value. The variant combination T430A/D showed the predicted pattern described for putative phosphorylation sites where the Dvariant is less active then the A-variant. The same pattern was detectable for the T500A/D combination but here the activity of the A-variant was about $50 \%$ reduced compared to the wild type enzyme whereas the D-variant was inactive. The combinations S409A/D and S598A/D were overall strongly reduced in activity and did not display the expected activity pattern (Fig. 3.15). 


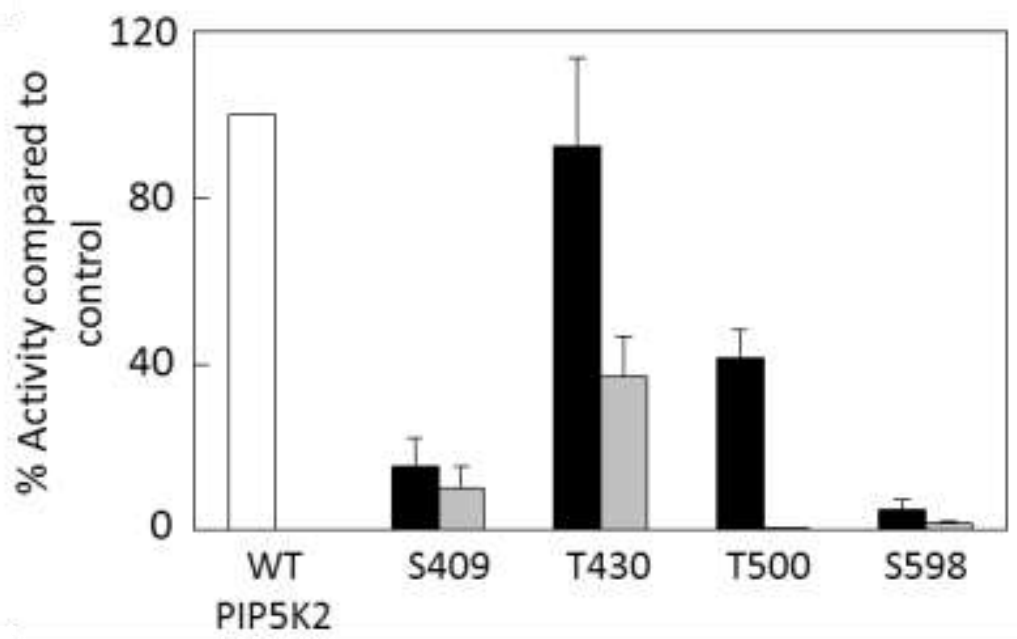

Fig. 3.15: PI4P 5-kinase activity of A/D variants of MBP-PIP5K2. Recombinant variants of MBP-PIP5K2 in which experimentally verified phosphorylation sites were altered to A or D were expressed in $E$. coli. Equal protein amounts of crude extract were tested for catalytic activity by adding Ptdlns4P and radiolabeled $\mathrm{V}-\left[{ }^{32} \mathrm{P}\right]$ ATP. Lipids were extracted, separated by TLC and radiolabeled PtdIns $(4,5) \mathrm{P}_{2}$ was quantified by a phosphorimager. Graphic shows activity of A- (black) and D- (grey) variants compared to wild type (wt) PIP5K2 (white) and represents means \pm SD of 2 experiments.

The characterization of phosphorylation sites that were chosen for mutation based only on computer aided prediction was performed accordingly and is shown in Figure 3.16. The PIP5K2 modifications S370A, S541A and T546A were more active than their corresponding D-Variants and thus supported the hypothesis. However, the activity even of the A-variants only reached 30-50 \% compared to the PIP5K2 control. The combination S475A/D was active about 40-50 \% (Fig. 3.16).



Fig. 3.16: PI4P 5-kinase activity of A/D variants of MBP-PIP5K2. Recombinant variants of MBP-PIP5K2 in which experimentally verified phosphorylation sites were altered to $A$ or $D$ were expressed in $E$. coli. Equal protein amounts of crude extract were subjected to activity tests by adding Ptdlns4P and radiolabeled $\mathrm{V}-\left[{ }^{32} \mathrm{P}\right]$ ATP. Lipids were extracted, separated by TLC and radiolabled PtdIns $(4,5) \mathrm{P}_{2}$ was quantified by a phosphorimager. Graphic shows activity of A- (black) and D- (grey) variants compared to wild type (wt) PIP5K2 (white) and represents means \pm SD of 2 experiments. 


\subsection{Degree of in vitro phosphorylation of PIP5K2 T430A and PIP5K2 T500A}

The determination of phosphorylation sites by biochemical and bioinformatical approaches described in the previous section has revealed several candidate phosphorylation sites that were altered to $A$ and $D$ and tested for catalytic activity. Importantly, in these experiments, the combinations T430A/D and T500A/D displayed high activity for the A-variant and low activity for the D-variant as suggested for the effects of phosphorylation on enzyme activity and were chosen for further analysis. As the other investigated combinations either did not show the expected difference between the A- and D-variants in activity or their ability to produce Ptdlns $(4,5) \mathrm{P}_{2}$ was overall strongly reduced, these variants were not considered any further. As the A-mutation of a specific amino acid mimics the constantly dephosphorylated state of an enzyme, it should not be open to phosphorylation any more. In a next experiment it was therefore tested whether the selected A-variants of PIP5K2 (MBP-PIP5K2 T430A and MBPPIP5K2 T500A) were still a substrate for in vitro phosphorylation.

MBP-PIP5K2 and the respective variants T430A and T500A were recombinantly expressed in E. coli and purified using affinity chromatography. Equal amounts of purified protein were incubated with radiolabled $y-\left[{ }^{32} \mathrm{P}\right]$ ATP and Arabidopsis leaf extract (see section 2.11.1). To exclude possible autophosphorylation MBP-PIP5K2 and the corresponding A-variants were incubated with $\gamma-\left[{ }^{32} \mathrm{P}\right]$ ATP in the absence of leaf extract. After incubation proteins were separated by SDS-PAGE, and phosphorylated bands were visualized using a phosphorimager (Fig. 3.17 A top). As depicted in Figure 3.17 A (top) both MBP-PIP5K2 and the variants T430A and T500A were still phosphorylated by an Arabidopsis-endogenous protein kinase. Autophosphorylation was not detected. To determine the relative $\mathrm{P}^{32}$-incorporation the intensity of phosphorylated MBP-PIP5K2, MBP-PIP5K2 T430A and MBP-PIP5K2 T500A was divided by the intensity of the respective Coomassie stained protein band. The normalized phosphorylation-value of each variant was then compared to that of MBP-PIP5K2 (Fig. 3.17 white bar) as depicted in Figure 3.17 B. After treatment with Arabidopsis leaf extract the phosphorylation-value of the MBP-PIP5K2 variant T430A decreased by about $50 \%$ compared to the wild type control. In confront, MBP-PIP5K2 T500A displayed stronger phosphorylation after treatment compared to wild type MBP-PIP5K2. 
A
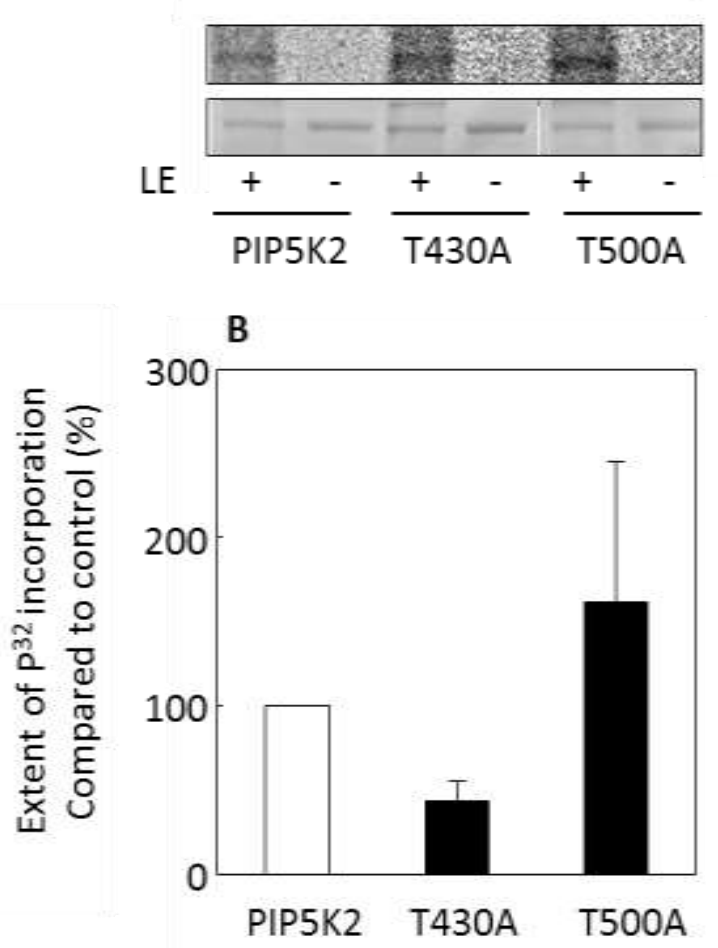

Fig. 3.17: In vitro phosphorylation of MBP-PIP5K2 T430A and MBP-PIP5K2 T500A. MBP-PIP5K2 T430A and MBP-PIP5K2 T500A were tested for phosphorylation by applying radiolabeled $\mathrm{Y}-\left[{ }^{32} \mathrm{P}\right]$ ATP and Arabidopsis leaf extract (LE). Proteins were separated by SDS-PAGE and phosphorylated protein bands were visualized by phosphorimaging. A, section of a phosphorimage (top) and the corresponding Coomassie stained gel (bottom). B, the level of phosphorylation of MBP-PIP5K2 T430A and MBP-PIP5K2 T500A was quantified and compared to the wild type control (white bar). The gels are replicates of two independent experiments and show similar results. The data are means of two independent experiments \pm SD.

Although the data described in this section further emphasize the role of phosphorylation in the functionality of PIP5K2, it should be noted that all results presented so far were based on in vitro experiments. In a next experiment the determined phosphorylation sites PIP5K2 T430 and T500 were therefore tested for in vivo effects.

\subsection{Plasma membrane-association of fluorescence-tagged PIP5K2 A/D-variants transiently expressed in tobacco pollen tubes}

PI4P 5-kinases are lipid modifying enzymes and thus associate to plant plasma membranes in order to convert Ptdlns4P to Ptdlns $(4,5) \mathrm{P}_{2}$. As described in the introduction it is suggested that phosphorylation of specific amino acids at the membrane interface prevent PI4P 5-kinases from correct localization by disturbing the electrostatic interactions between lipid kinase and target membrane. Therefore, in the next section it was tested whether the A/D substitutions at determined phosphorylation sites had effects on the membrane localization of PIP5K2. 
Plasma membrane localisation was monitored by fusing PIP5K2 and the respective variants PIPK2 T430A/D and PIPK2 T500A/D to a C-terminal enhanced yellow fluorescent protein (EYFP) and transiently expressing the fusions in tobacco pollen tubes. Membrane association was investigated after $7 \mathrm{~h}$ of growth in pollen tubes showing vigorous cytoplasmic streaming and is depicted in Figure 3.18.

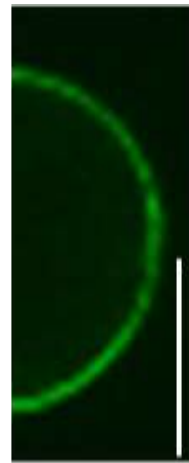

Wild type

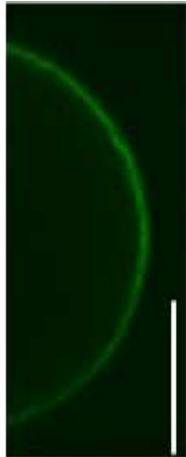

T500A



T500D

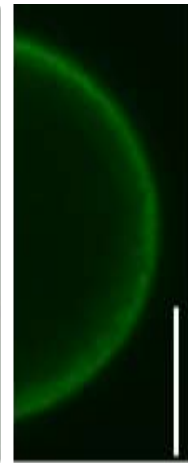

T430A

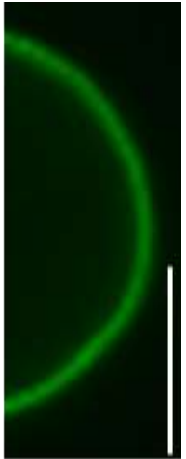

T430D

Fig. 3.18: Apical plasma membrane association of PIP5K2-EYFP wild type and corresponding A/Dvariants in tobacco pollen tubes. Tobacco pollen tubes were transformed with PIP5K2-EYFP (wt), PIP5K2 T500A-EYFP, PIP5K2 T500D-EYFP, PIP5K2 T430A-EYFP and PIP5K2 T430D-EYFP. Pollen tubes were grown for $7 \mathrm{~h}$ and PIP5K2-EYFP localization was imaged by confocal microscopy. Bars, $10 \mu \mathrm{m}$. Images are representatives for about 100 transformed pollen tubes per construct.

Transient expression of wild type PIP5K2-EYFP displayed a characteristic localization at the apical tip of the tobacco pollen tube (Fig. 3.18 left) as has already been described in the introduction. The localization of fluorescence tagged variants PIP5K2 T500A-EYFP and PIP5K2 T500D-EYFP was similar to each other and showed no difference to the localization of wild type PIP5K2-EYFP (Fig. 3.18). These observations indicate that the effect of phosphomimicry on catalytic activity is not related to the displacement of the enzyme from the membrane.

\subsection{Effects of PIP5K2 A/D variants on pollen tube morphology}

So far the analysis of selected A/D-variants of PIP5K2 provided information about their in vitro PI4P 5-kinase activity and their subcellular localization in tobacco pollen tubes. Subsequent experiments were performed to test the in vivo functionality of the PIP5K2 variants. A characteristic effect caused by the heterologous expression of Arabidopsis PIP5K2 in tobacco pollen tubes is a change in their morphology and tip-swelling as has previously been described (Stenzel et al., 2012). As indicated in Figure 3.19 pollen tubes expressing PIP5K2 can be classified in two morphological groups "normal growth" representing pollen tubes with 0$15 \mu \mathrm{m}$ in diameter (Fig. 3.19 A) and "apical tip-swelling" representing pollen tubes exceeding $15 \mu \mathrm{m}$ in diameter (Fig. 3.19 B). Apical tip-swelling was detected for the majority of the 
investigated pollen tubes upon expression of PIP5K2-EYFP, and only $30 \%$ showed a normal growing phenotype (Fig. 3.19 C). Tobacco pollen tubes expressing EYFP as a negative control exhibited no apical tip-swelling and grew normally (Fig. 3.19 C). These data are consistent with observations reported previously (Stenzel et al., 2012).

A

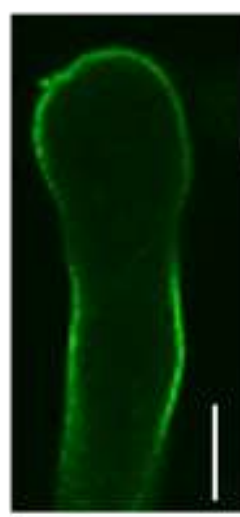

Normal

growth
B

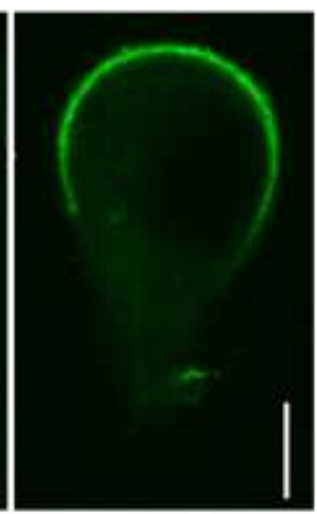

Apical tip swelling



Fig. 3.19: Effects of PIP5K2-EYFP expression on the morphology of tobacco pollen tubes. PIP5K2-EYFP was transiently expressed in tobacco pollen tubes and effects on morphology were monitored after $12 \mathrm{~h}$ of growth. Two different phenotypes can be observed after PIP5K2-EYFP expression: normal growing pollen tubes (A) or apical tip-swelling (B). Bars, $10 \mu \mathrm{m}$. The phenotypic distribution was investigated by measuring the diameter of transgenic pollen tubes (C). Black bars represent pollen tubes expressing PIP5K2-EYFP white bars show negative control expressing EYFP alone. Images are representative for $>300$ pollen tubes. Data display means \pm SD of 6 independent transformation experiments with at least 100 pollen tubes each. The asterisks indicate a significant difference between normal growth and apical tip-swelling after expression of PIP5K2-EYFP according to a student's t-test $(p<0.001)$.

Subsequent experiments are based on the assumption that functionally active PIP5K2 might result in a higher proportion of swollen pollen tube tips whereas less active or inactive PIP5K2 would not alter pollen tube morphology, resulting in a higher proportion of normal growing pollen tubes. Based on this hypothesis PIP5K2 T430A/D and PIP5K2 T500A/D were transiently expressed in tobacco pollen tubes and the cells analyzed for the arising morphological phenotypes (Fig. 3.20). Figure 3.20 A shows the phenotypic distribution of tobacco pollen tubes expressing PIP5K2-EYFP (black bar) and the variants T430A (white bar) T430D (grey bar). The transient expression of PIP5K2 T430A-EYFP and PIP5K2 T430D-EYFP both increased the amount of pollen tubes with more than $15 \mu \mathrm{m}$ in diameter. More than $60-70 \%$ of investigated pollen tubes displayed tip-swelling upon expression of PIP5K2 T430A-EYFP and PIP5K2 T430DEYFP, respectively (Fig. 3.20 A). Similar results were observed when pollen tubes were transformed with PIP5K2-EYFP as control. Figure $3.20 \mathrm{~B}$ illustrates the morphology of pollen tubes expressing PIP5K2 T500A-EYFP (white bar) and PIP5K2 T500D-EYFP (grey bar). After expression of PIP5K2 T500A-EYFP about $75 \%$ of the analyzed pollen tubes displayed a swollen 
tip as has been shown for the control PIP5K2-EYFP construct (Fig. 3.21 B black bar). In contrast, pollen transformed with PIP5K2 T500D-EYFP displayed normal growth (Fig. 3.21 B grey bar).


Fig. 3.20: Effects of expressing variants of PIP5K2-EYFP on the morphology of tobacco pollen tubes. PIP5K2-EYFP and PIP5K2-EYFP variants were transiently expressed in tobacco pollen tubes and effects on morphology were monitored after $12 \mathrm{~h}$ of growth. The phenotypic distribution was investigated by measuring the diameter of transgenic pollen tubes. A, black bar, PIP5K2-EYFP; white bar, PIP5K2 T430AEYFP; grey bar, PIP5K2 T430D-EYFP. B, black bar, PIP5K2-EYFP; white bar, PIP5K2 T500A-EYFP; grey bar, PIP5K2 T500D-EYFP. Data display means \pm SD of 3-6 independent experiments with each representing at least 100 pollen tubes. The asterisks indicate a significant difference between normal growth and apical tip-swelling after expression of PIP5K2 T500D-EYFP compared to control PIP5K2-EYFP constructs according to a student's t-test $(p<0.001)$.

\subsection{Effects of overexpression of PIP5K2 variants in tobacco pollen tubes are not correlated with different expression levels}

In the last section the in vivo activity of PIP5K2 T500A/D and PIP5K T430A/D combinations was tested by heterologous expression in tobacco pollen tubes. Tobacco pollen tubes expressing PIP5K2 T430A-EYFP, PIP5K2 T430D-EYFP and PIP5K2 T500A-EYFP depicted apical tip swelling as has been described for PIP5K2-EYFP (Stenzel et al., 2008) and thus, were thought to be functional in vivo. In contrast, expression of PIP5K2 T500D-EYFP had no influence on tobacco pollen tube morphology suggesting that this PIP5K2 variant was catalytic inactive in vivo. To test whether higher expression levels account for the observed morphological changes in pollen tubes expressing PIP5K2 T430A-EYFP, PIP5K2 T430D-EYFP and PIP5K2 T500A-EYFP compared to PIP5K2 T500D-EYFP, relative fluorescence intensities of transformed pollen tubes were measured with ImageJ (http://rsbweb.nih.gov/ij/). 
Figure 3.21 depicts the relative fluorescence intensity patterns for pollen tubes expressing the indicated PIP5K2-EYFP variants and shows that the amount of pollen tubes decreases continually towards high fluorescence intensities for all of the investigated constructs. About 40 to $50 \%$ of the pollen tubes transformed with PIP5K2-YFP, PIP5K2 T430A-EYFP and PIP5K2 T430D-EYFP constructs display low fluorescence intensities (Fig. 3.21 1-20). Pollen tubes heterologously expressing PIP5K2 T500A-EYFP and PIP5K2 T500D-EYFP are equally distributed between low and medium fluorescence intensities (Fig. 3.21 1-60). Although the fluorescence intensity of pollen tubes expressing PIP5K2 T500A-EYFP and PIP5K2 T500D-EYFP was similar, their effect on tobacco pollen tube morphology was severely different (Fig. 3.20 B), suggesting that the observed pollen tube phenotypes were not a result of altered expression level but indeed depended on the in vivo catalytic activity of the investigated PIP5K2-EYFP variants. An increased proportion of pollen tubes expressing PIP5K2 T500A-EYFP at high intensity was observed for $20 \%$ of the pollen subjected to analysis and may contribute to the wild type-like pattern illustrated for that variant in Figure 3.20. As the high intensity is only observed in $20 \%$ of the pollen tubes tested, this effect is not likely to substantially impair interpretation of the data shown in Figure 3.20.

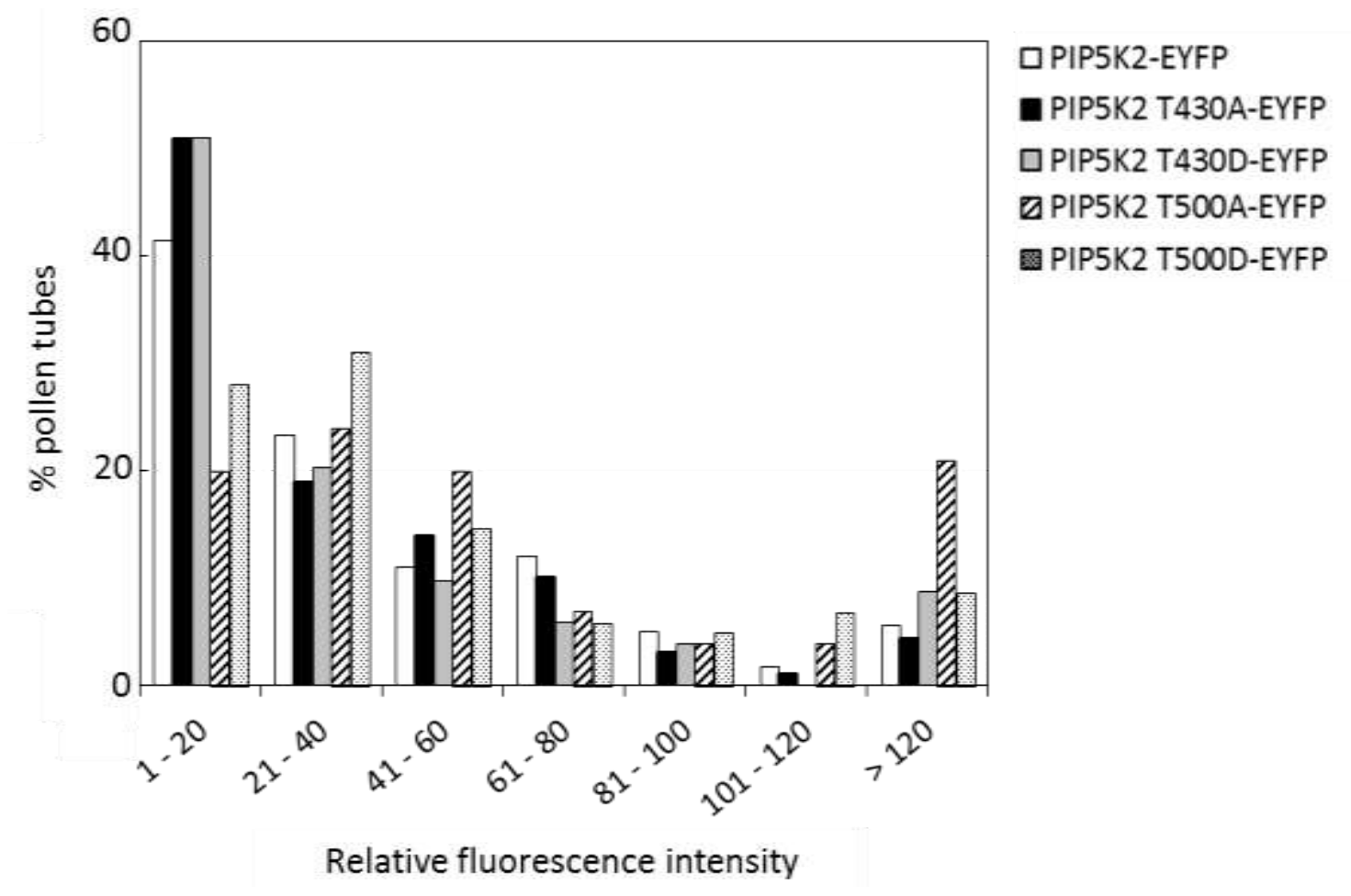

Fig. 3.21: Fluorescence intensities of pollen tubes expressing PIP5K2-EYFP and the corresponding variants. PIP5K2-EYFP and variants were transiently expressed in tobacco pollen tubes and the relative fluorescence was measured after $12 \mathrm{~h}$ of growth. Over 100 pollen tubes per construct were analysed with light exposure of $0.2 \mathrm{~s}$. 


\subsection{11 Mutant complementation}

Although the heterologous expression in tobacco pollen tubes gave first hints towards the in vivo functionality of PIP5K2 T430 A/D and PIP5K2 T500A/D combinations, it was next tested whether the investigated variants can also function in their homologous system. PIP5K2 is ubiquitously expressed in Arabidopsis, and although it has been postulated that Arabidopsis pip5k2 mutants show reduced lateral root formation (Mei et al., 2012), this phenotype was not observed during experiments performed in this thesis (data not shown). The disruption of an additional PI4P 5-kinase, PIP5K1, results in an Arabidopsis pip5k1 pip5k2 double mutant that has a severe phenotype as already described in section 2.17. By expressing PIP5K2 variants in the pip5k1 pip5k2 double mutant background and investigating the resulting phenotype, qualitative conclusions about the in vivo catalytic activity can be made.

To test whether the PIP5K2 variants investigated so far were able to complement the pip5k1 pip5k2 double mutant phenotype, PIP5K2 cDNA was expressed in the heterozygous pip5k1 PIP5K1 x pip5k2 pip5k2 mutant line under the control of a 1500-bp PIP5K2 promoter fragment as a fusion to a C-terminal EYFP tag. After transformation, the offspring was screened for pip5k1 $\times$ pip5k2 PIP5K2-EYFP mutants by amplifying the T-DNA tagged pip5k1 and pip5k2 alleles and the corresponding wild type alleles (Fig. 3.22 E). As can be seen in Figure 3.22 E, amplification of PIP5K1 and PIP5K2 wild type alleles results in a band with a size of $1200 \mathrm{bp}$. PIP5K2 wild type alleles were also amplified in pip5k1 $x$ pip5k2 PIP5K2 T500A-EYFP and pip5k1 x pip5k2 PIP5K2 T500D-EYFP mutant plants but were only about $750 \mathrm{bp}$ in size. The local amplification of the genomic PIP5K2 sequence contains two introns that are absent from PIP5K2-EYFP cDNA used for transformation experiments (Fig. 3.22 F). The observed size difference after amplification of the PIP5K2 wild type allele in pip5k1 1 × pip5k2 PIP5K2 T500AEYFP and pip5k1 $\times$ pip5k2 PIP5K2 T500D-EYFP mutant plants therefore is connected to the general absence of introns in cDNA and confirms that the transformation was successful. pip5k1 $x$ pip5k2 PIP5K2 T500A-EYFP and pip5k1 $x$ pip5k2 PIP5K2 T500D-EYFP were phenotypically analyzed (Fig. 3.22). Figure $3.22 \mathrm{~A}$ and $\mathrm{C}$ show wild type and pip5k1 pip5k2 double mutant plants, respectively. The expression of PIP5K2 T500A-EYFP in the pip5k1 pip5k2 double mutant background is able to rescue the phenotype (Fig. 3.22 B). pip5k1 x pip5k2 PIP5K2 T500A-EYFP mutants grew like wild type plants and also developed normal inflorescence. In contrast to that, pip5k1 x pip5k2 PIP5K2 T500D-EYFP mutant plants depicted the same phenotype as described for pip5k1 pip5k2 double mutants (Fig. $3.22 \mathrm{D}$ ). 

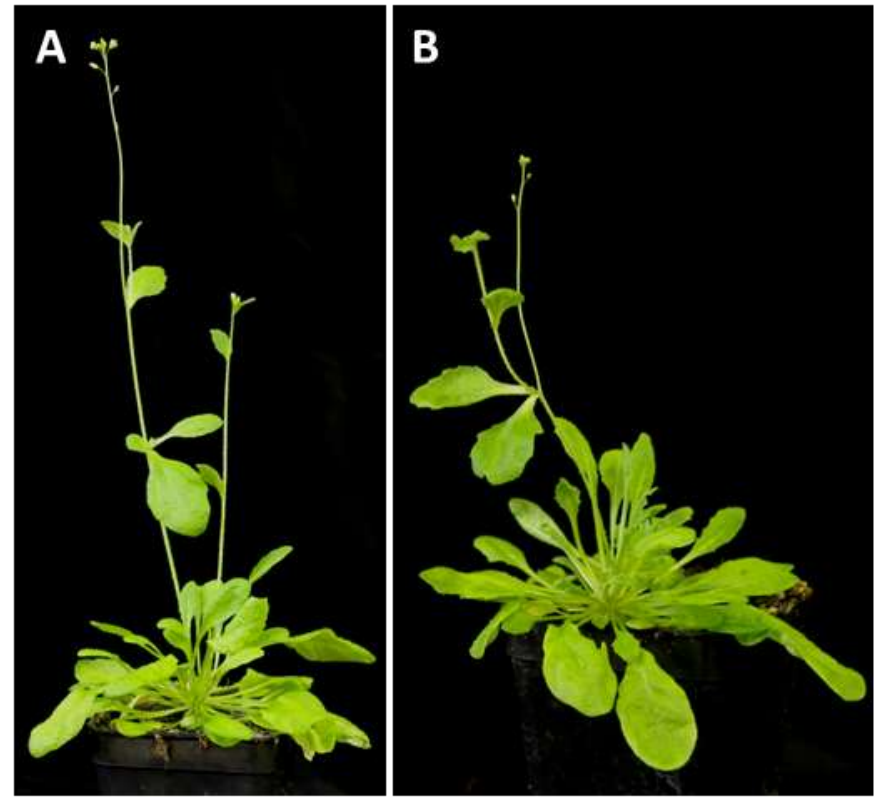

\section{E Wild type}

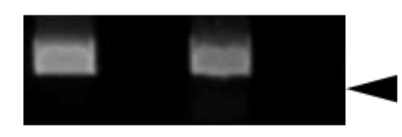

pip5k1

pip $5 k 2$



T500A

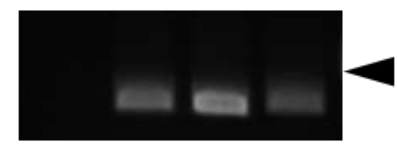

T500D

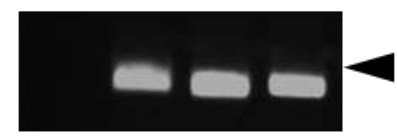

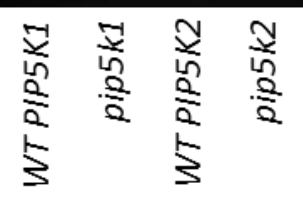



$\mathbf{F}$

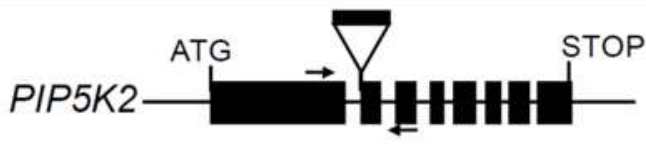

Fig. 3.22: Expression of PIP5K2-EYFP T500A can rescue the phenotype of the Arabidopsis pip5k1 pip5k2 double mutant. PIP5K2::PIP5K2T500A:EYFP and PIP5K2::PIP5K2T500D:EYFP cDNA was expressed in the pip5k1 PIP5K1 pip5k2 pip5k2 mutant background. Transformed double mutant plants identified by PCR-based genotyping and analyzed for morphology. A, Wild type control. B, Double mutant expressing PIP5K2T500A-EYFP. C, Double mutant control displaying dwarfism. D, Double mutant expressing PIP5K2T500D-EYFP. All plants shown (A-D) have been grown side-by-side for the same period under identical conditions. E, PCR based genotyping. Black arrowheads indicate $1000 \mathrm{bp}$. F, genomic PIP5K2 sequence with exons (black) and introns. The white arrowhead indicates the site if the T-DNA insertion.

The PIP5K2 variants of T500 were selected for the mutant complementation tests because of the clear difference in catalytic activities between T500A and T500D (Fig. 3.15). In future experiments, it will be interesting to also test the effects of expressing PIP5K2 T430A and PIP5K2 T430D in the Arabidopsis pip5k1 pip5k2 mutant background.

\subsection{Effects of salt stress on the phosphorylation status of PIP5K2}

Previous reports have suggested that salt stress-induced increases of Ptdlns $(4,5) \mathrm{P}_{2}$ may have a role in membrane maintenance and the adaptation of plants to salt-stress. Increased formation of Ptdlns $(4,5) \mathrm{P}_{2}$ after application of salt stress occurs very rapidly and suggests an 
effective regulatory mechanism for PI4P 5-kinases for example by posttranslational modification. During this work it was confirmed that protein phosphorylation is one regulatory factor of PIP5K2 activity. Two phosphorylation sites, T430 and T500, were identified and have been characterized as negative regulators of PIP5K2 activity. The data so far indicate that stress-induced activation of PIP5K2 involves dephosphorylation of previously existing, inactive phospho-enzyme.

To test for effects of plant extracts challenged with hyperosmotic stress on the phosphorylation status of PIP5K2, two week old plants were treated with $0.4 \mathrm{M} \mathrm{NaCl}$ and extracts were prepared after 15, 30 and 60 min of salt stress as described in 2.11. Control extracts were also prepared prior to the treatment. Afterwards purified MBP-PIP5K2 was incubated with equal concentrations of the respective leaf extracts, samples were separated via SDS-PAGE and phosphorylated proteins were visualized by phosphorimaging. Prior to stress treatment leaf extract was able to phosphorylate MBP-PIP5K2 (Fig. 3.23). After 15 min of salt stress leaf extract still phosphorylated MBP-PIP5K2 to $100 \%$. Extracts prepared from plants treated with $\mathrm{NaCl}$ for 30 and 60 min also had no adverse effect on the phosphorylation status of MBP-PIP5K2, indicating that protein kinase activity was unabated in those extracts. The experimental setup does not enable the detection of PIP5K2 dephosphorylation, as might indicate stress-induced activation of the enzyme, because of the necessary presence of phosphatase inhibitors in the assay mix. The data are thus consistent with the notion that PIP5K2-activity is inhibited, not activated by reversible phosphorylation.

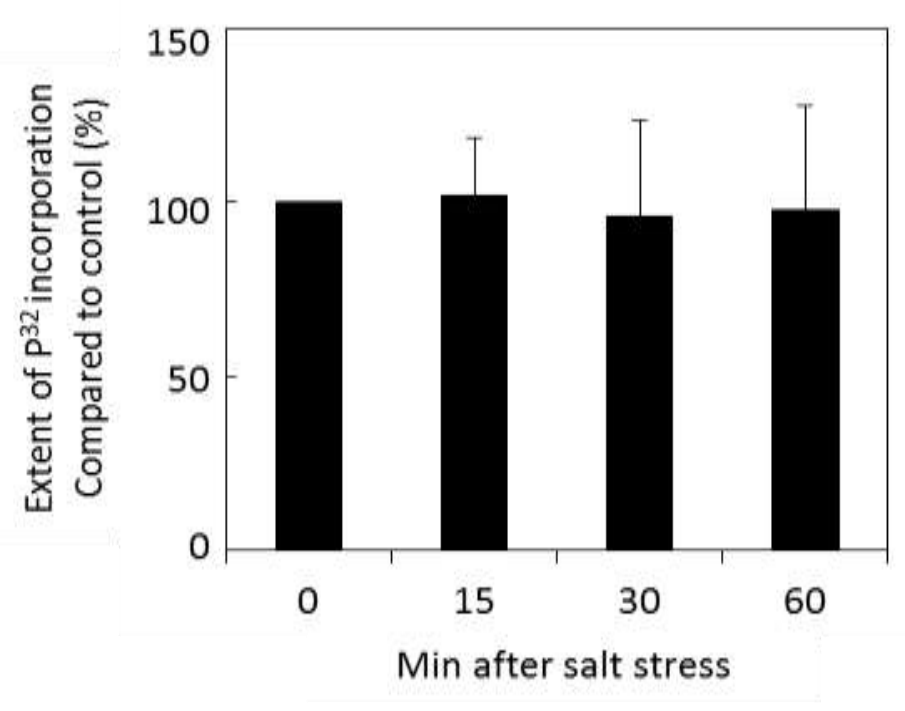

Fig. 3.23: MBP-PIP5K2 is equally phosphorylated by plant extracts challenged with salt stress. Plant extracts were prepared prior and after hyperosmotic stress and used to phosphorylate purified MBPPIP5K2. Results are triplicates \pm SD. 


\subsection{Similar or different? Control of PIP5K3 activity by reversible phosphorylation}

The results described in the previous sections suggest an important role for reversible phosphorylation in the control of PIP5K2 functionality. In the context of investigating possible phosphorylation sites of Arabidopsis PIP5K2, the online database PhosPhAT 4.0 revealed an experimentally verified phosphorylation site at position T472 for another PI4P 5-kinase isoform from Arabidopsis, PIP5K3 (see section 3.4.1). PIP5K3 T472 is highly conserved in the Arabidopsis PI4P 5-kinase family. Importantly, altering the corresponding amino acid T500 in PIP5K2 has resulted in severe effects on PIP5K2 in vitro (Fig. 3.15) and in vivo activity (Figs. 3.20-3.22), raising the question whether such observations could also be verified for PIP5K3. As the most active PI4P 5-kinase encoded in the Arabidopsis genome (Stenzel et al., 2008), PIP5K3 represents an important candidate and was thus included in additional studies.

\subsection{PIP5K3 can be recombinantly expressed in $E$. coli}

To test possible effects of phosphorylation on Arabidopsis PIP5K3 functionality it was necessary to recombinantly express and purify PIP5K3 as has been shown for MBP-PIP5K2 in section 3.1. Similar to PIP5K2, PIP5K3 was expressed as a fusion to an $\mathrm{N}$-terminal MBP tag in E. coli Rosetta 2 cells (for details see section 2.2.4) and purified via affinity chromatography using an MBP trap matrix. The plasmids were provided by Dr. Irene Stenzel (MLU HalleWittenberg). Enrichment was monitored by applying each collected fraction onto an SDS-PAGE (Fig. 3.24). As illustrated in Fig. $3.23 \mathrm{~A}$, the amount of Coomassie-stained protein decreased with the progress of purification. In the first eluted fraction no protein bands of the expected size were detectable, in the second eluted fraction a protein band at $120 \mathrm{kDa}$ appeared that was consistent with the calculated size of MBP-PIP5K3 full-length protein. In addition to the putative MBP-PIP5K3 band, smaller but still distinct bands were also detectable in the second eluted fraction. To verify enrichment of MBP-PIP5K3 an immunoblot using an MBP antibody was performed (Fig. 3.24 B). Although amount and intensity of specifically detected protein bands in the crude extract and the second eluted fraction were comparable, the MBP antibody visualized a protein band of $120 \mathrm{kDa}$ size in the second eluted fraction which was not as pronounced in the crude extract or the other applied fractions (Fig. 3.24 B). 


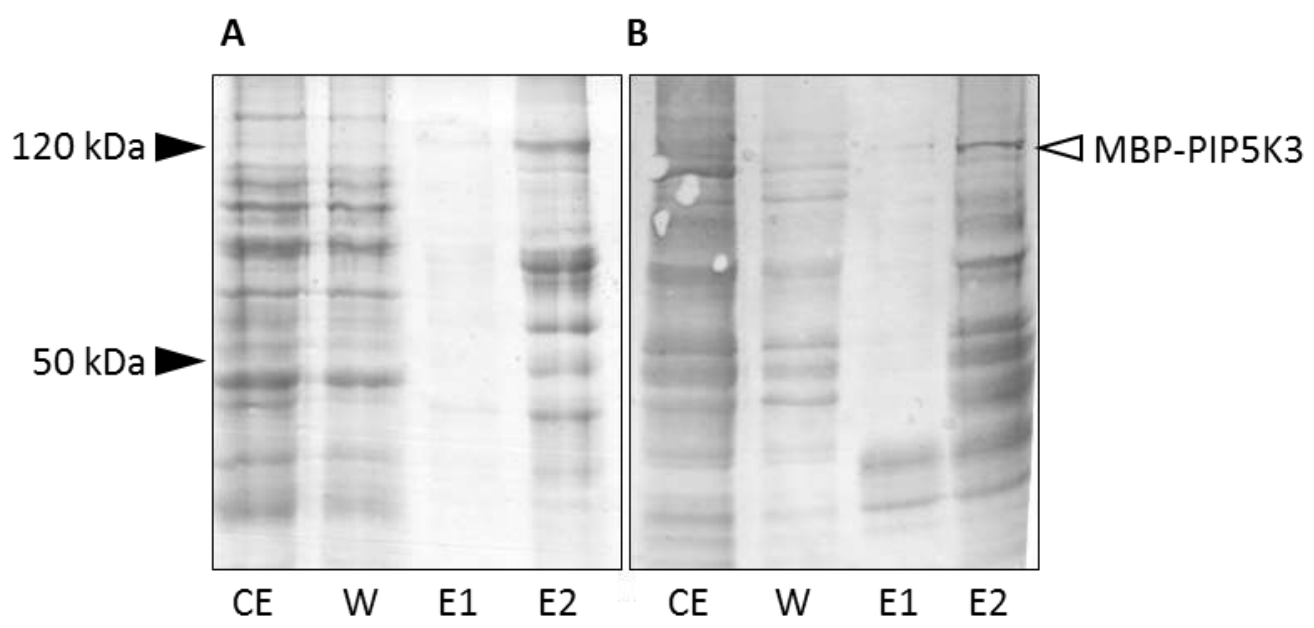

Fig. 3.24: Recombinant expression and purification of PIP5K3. MBP-PIP5K3 was heterologously expressed in E. coli Rosetta 2 cells and purified with an MBP column. The progress of purification was determined by a Coomassie stained SDS-PAGE-gel (A) and the respective immunoblot using an MBP antibody (B). CE, crude extract; W, wash; E1, eluted fraction 1; E2, eluted fraction 2. Black arrows indicate protein sizes defined by a commercial protein marker and apply for A and B; white arrows show the size of the full-length MBP-PIP53 fusion construct. The experiment was repeated twice \pm SD with similar results.

To test if purified MBP-PIP5K3 was still active after enrichment equal protein concentrations of crude extract and second eluted fraction were analysed for their ability to form $\operatorname{Ptd} \operatorname{lns}(4,5) \mathrm{P}_{2}$. Equal protein amounts were incubated with PtdIns4P and radiolabeled $\gamma-\left[{ }^{32} \mathrm{P}\right]$ ATP, lipid products were separated by $\mathrm{TLC}$ and radiolabeled $\operatorname{Ptd} \operatorname{lns}(4,5) \mathrm{P}_{2}$ was quantified by phosphorimaging. Figure 3.25 illustrates the amount of Ptdlns $(4,5) \mathrm{P}_{2}$ synthesized in pmol per minute per mg protein and shows that the specific activity of crude extract was about $30 \%$ higher compared to the second eluted fraction.

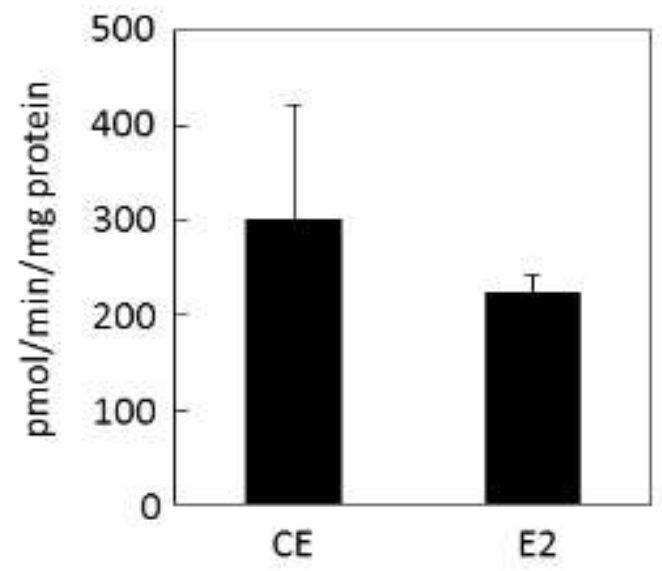

Fig. 3.25: Specific activity of recombinantly expressed MBP-PIP5K3. Crude extracts (CE) and the second eluted fraction (E2) were tested for activity by applying Ptdlns4P and $\mathrm{\gamma}-\left[{ }^{32} \mathrm{P}\right]$ ATP as substrates. Product lipids were extracted and separated by TLC. The amount of radiolabled Ptdlns $(4,5) \mathrm{P}_{2}$ was quantified by a phosphorimager. Data represent the means of two independent experiments. 
As summarized in Table 3.4 protein amount and overall activity decreased severely during purification. Based on the specific activity determined in the previous experiment a purification factor of 0.75 was calculated, suggesting that MBP-PIP5K3 activity was rather depleted than enriched. Although the purification factor was smaller than 1, the Coomassie stained gel and immunoblot (Fig. 3.24 A and B) both showed a substantially concentrated band at $120 \mathrm{kDa}$ in the second eluted fraction. Therefore the heterologously expressed and purified MBP-PIP5K3 was used for further applications. The reduced activity will be discussed further at a later point.

Table 3.4: Purification table of affinity enriched MBP-PIP5K3. Data show the progress of purification by relating the specific activity of crude extract with the specific activity of the second eluted fraction.

\begin{tabular}{ccccccc}
\hline Step & $\begin{array}{c}\text { Volume } \\
\text { (ml) }\end{array}$ & $\begin{array}{c}\text { Protein } \\
\text { (mg) }\end{array}$ & $\begin{array}{c}\text { Activity } \\
\text { (pmol/min) }\end{array}$ & $\begin{array}{c}\text { Specific activity } \\
\text { (pmol/min/mg) }\end{array}$ & $\begin{array}{c}\text { Yield } \\
\text { (\%) }\end{array}$ & $\begin{array}{c}\text { Purification } \\
\text { (factor) }\end{array}$ \\
\hline $\begin{array}{c}\text { Crude } \\
\text { Extract }\end{array}$ & 10 & 54 & 16259.4 & 301.1 & 100 & 1 \\
Elution 2 & 1 & 0.71 & 159.97 & 225.31 & 1 & 0.75 \\
\hline
\end{tabular}

\subsection{Phosphorylation of recombinantly expressed and purified PIP5K3}

The identification of the PIP5K3 residue T472 as a phosphorylation site indicates that posttranslational modification not only occurs on Arabidopsis PIP5K1 and PIP5K2 but possibly plays an important function in regulating the whole Arabidopsis PI4P 5-kinase family. In analogy to experiments performed with PIP5K2 (see section 3.2.1) purified MBP-PIP5K3 was subjected to phosphorylation by PKA and relevant plant extracts respectively as will be described in the following sections.

\subsubsection{Recombinant PIP5K3 is phosphorylated by PKA in vitro}

To test for in vitro phosphorylation $10 \mu \mathrm{g}$ purified MBP-PIP5K3 was incubated with commercially available PKA and radiolabeled $\mathrm{y}-\left[{ }^{32} \mathrm{P}\right]$ ATP as described in section 2.11 Protein phosphorylation was detected by separating the samples via SDS-PAGE and exposing a radiosensitive film. Figure 3.26 shows a section of a Coomassie stained gel (A) as a loading control and the corresponding autoradiograph (B). As depicted in the autoradiograph (Fig. 3.26 B) a radiolabeled MBP-PIP5K3 full-length protein (lane 1, white arrowhead) was only weakly detectable due to the high background. In the absence of exogenous PKA the background disappeared. No autophosphorylation was observed (Fig. 3.26 B lane 3). Similar to experiments performed with MBP-PIP5K2, MBP-PIP5K3 was cleaved with TEV protease, to exclude that the 
detected protein phosphorylation was attributed to phosphorylation of the MBP tag (Fig. 3.26, lane 2). As can be seen in the loading control (Fig. 3.26 A lane 2) the TEV cleavage of MBPPIP5K3 was incomplete. However, a weak radiolabeled signal was detectable in the corresponding lane of the phosphorimage that correlated to the size of PIP5K3 without MBPtag (Fig. 3.26 B lane 2, black arrowhead). The reversibility of the phosphorylation was also tested by incubating PKA treated MBP-PIP5K3 with SAP. As shown in lane 3 (Fig. 3.26 B) the phosphorylated MBP-PIP5K3 protein band disappeared. No autophosphorylation was observed (Fig. 3.26 B, lane 4).

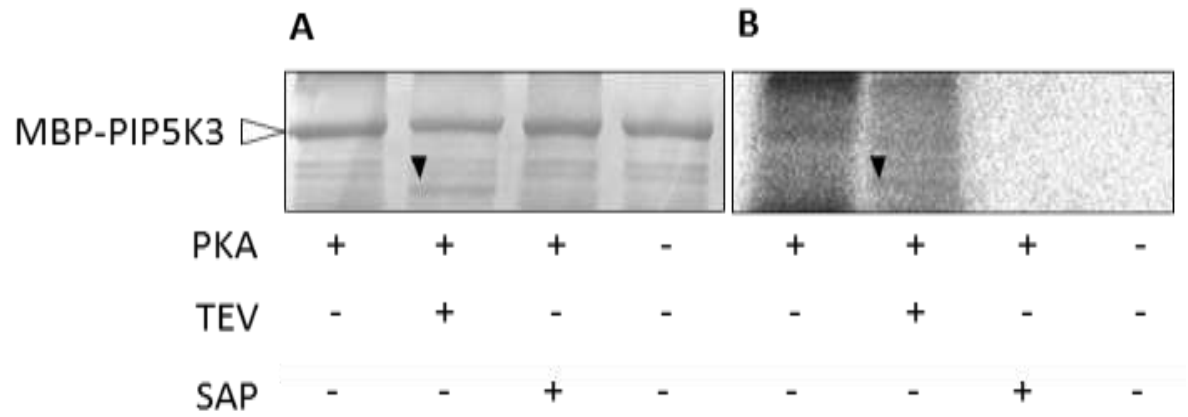

Fig. 3.26: Recombinantly expressed and purified PIP5K3 is phosphorylated by PKA. PIP5K3 was subjected to phosphorylation by incubating equal amounts of purified MBP-PIP5K3 with PKA and radiolabeled ${ }^{32} \mathrm{P}$ ATP. TEV protease and SAP were added as indicated. After incubation proteins were separated by SDS-Page and stained with Coomassie (A). B shows the respective phosphorimage. Black arrowheads indicate sizes of MBP-PIP5K3 fusion protein (lane 1) and PIP5K3 without MBP-tag (lane 2). Data are representative for two independent experiments.

The results indicate that recombinantly expressed MBP-PIP5K3 was phosphorylated by PKA. To investigate if phosphorylation of MBP-PIP5K3 can be repeated by an endogenous Arabidopsis protein kinase purified MBP-PIP5K3 was subjected to phosphorylation by an Arabidopsis plant extract.

\subsubsection{Purified PIP5K3 is phoshorylated by an endogenous Arabidopsis protein kinase}

In contrast to PIP5K2, PIP5K3 is predominantly expressed in Arabidopsis roots and seedlings (Stenzel et al., 2008). Therefore two-week-old seedlings were used as a source for preparing extracts containing relevant protein kinase activity. To test for phosphorylation, $10 \mu \mathrm{g}$ of purified MBP-PIP5K3 were incubated with seedling extract and radiolabled $\psi-\left[{ }^{32} \mathrm{P}\right]$ ATP. In addition, experiments were repeated with leaf extract of 6-week-old Arabidopsis plants to investigate potential differences in the phosphorylation status of PIP5K3. Phospholabeled proteins were separated by SDS-PAGE (Fig. 3.27 A) and visualized by a phosphoimager (Fig. 3.27 B). Figure 3.27 A illustrates the Coomassie stained gel and serves as loading control. The 
white arrowhead indicates full-length MBP-PIP5K3. Figure $3.27 \mathrm{~B}$ and $\mathrm{C}$ represent the respective phosphorimages and illustrate that both leaf extract (Fig. 3.26 B, lane 1) and seedling extract (Fig. 3.27 B, lane 2) phosphorylated the 120 kDa MBP-PIP5K3 full-length protein (black arrowheads). These bands were missing, when both extracts were applied without additional MBP-PIP5K3 (Fig. 3.27 C, black arrowheads) suggesting that they were not arising from the plant extracts. Furthermore, it was observed that leaf extract-treated MBPPIP5K3 ran slightly higher than seedling extract-treated MBP-PIP5K3.

A

B

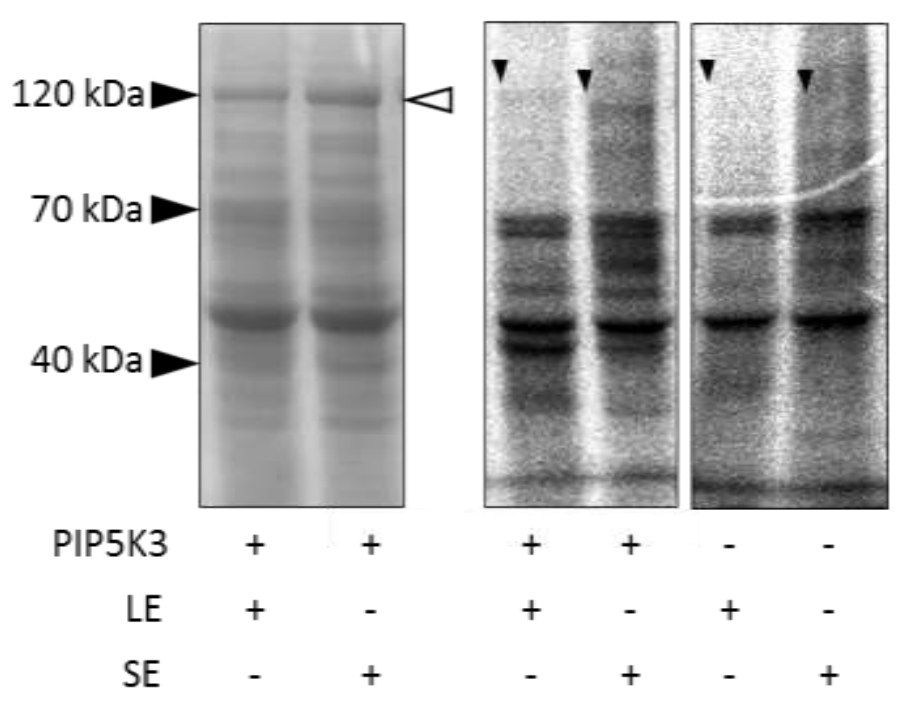

Fig. 3.27: Purified MBP-PIP5K3 is phosphorylated by different Arabidopsis extracts. Purified MBPPIP5K3 was tested for phosphorylation by applying radiolabeled $\gamma-\left[{ }^{32} \mathrm{P}\right]$ ATP and Arabidopsis leaf extract (LE) and Arabidopsis seedling extract (SE). Proteins were separated by SDS-PAGE and phosphorylated protein bands were visualized by phosphoimaging. A, Coomassie stained gel used as loading control. The white arrow marks the size of full-length MBP-PIP5K3. B and C, corresponding phosphorimages. Black arrows in B indicate the size difference between LE- and SE- treated MBP-PIP5K3. Black arrows in C indicate that plant extracts alone exhibit phosphorylated bands with this size. Images are triplicates and show similar results.

An attempt to treat phosphorylated MBP-PIP5K3 with TEV and test for phosphorylation of MBP by the plant extract was made but gave no interpretable results since phosphorylated bands comigrated with the intrinsic phosphorylated proteins of the plant extracts.

The data presented in the last section indicate that Arabidopsis PIP5K3 can be phosphorylated by PKA and Arabidopsis plant extracts in vitro. In the following section it was investigated whether phosphorylation of PIP5K3 altered PI4P5-kinase activity as has been shown for phosphorylated PIP5K2 in section 3.2. 


\subsection{In vitro phosphorylation of PIP5K3 reduces catalytic activity}

To test for changes in catalytic activity, purified MBP-PIP5K3 was pre-incubated with PKA and $5 \mathrm{mM}$ of cold ATP and subsequently analyzed for its ability to form Ptdlns $(4,5) \mathrm{P}_{2}$ as described before. The PI4P 5 kinase activity of PKA-treated MBP-PIP5K3 was then compared to the activity of a buffer-treated control (Fig. 3.28). The activity of PKA-treated MBP-PIP5K3 was reduced by $40 \%$ compared to the untreated control.

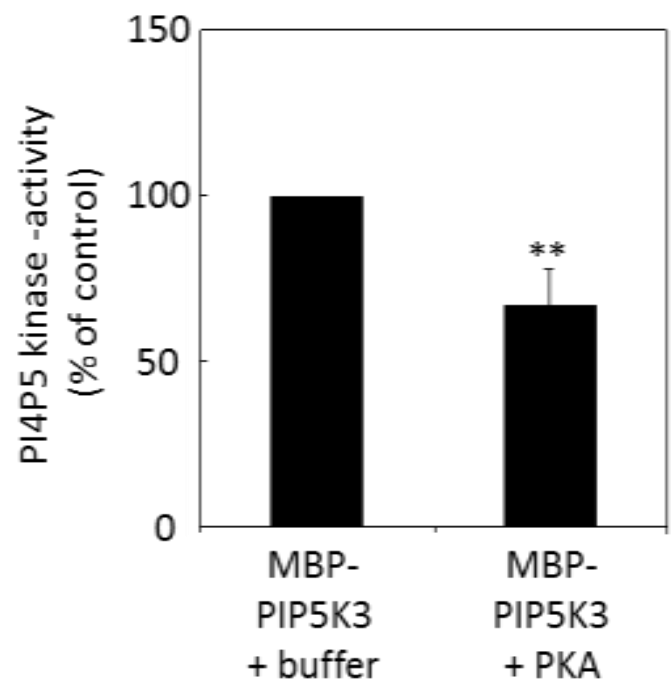

Fig. 3.28: PIP5K3 is inhibited by PKA. Purified MBP-PIP5K3 was subjected to PKA and cold ATP following activity tests with radiolabled $\gamma-\left[{ }^{32} \mathrm{P}\right]$ ATP and Ptdlns4P as substrates. The control sample was incubated in the same conditions with buffer instead of PKA. Data represent the means \pm SD of three independent experiments each assayed in duplicates. The asterisks indicate a significant decrease of PI4P 5-kinase activity after incubation with PKA compared to the buffer-treated control according to a student's t-test $(p<0.004)$.

To further test the influence of an Arabidopsis plant extract on PIP5K3 activity, equal amounts of purified recombinant MBP-PIP5K3 were treated with seedling extract in the presence of different concentrations of cold ATP. The ability to form Ptdlns $(4,5) P_{2}$ was then tested as described before (see section 2.10.9) and compared to the activity of a control treated with $5 \mathrm{mM}$ ATP and buffer instead of seedling extract (Fig. 3.29). To verify that MBP-PIP5K3 was not degraded upon treatment with seedling extract, an immunoblot was performed using an MBPantibody (Fig. 3.29). Differences in the MBP-PIP5K3 band intensities were taken into account. As illustrated in figure 3.29 incubation of MBP-PIP5K3 with seedling extract in the absence of endogenous ATP severely increased PI4P 5-kinase activity about 10-fold (please note the logarithmic scale in Figure 3.29). When seedling extract was supplied with 1 or $5 \mathrm{mM}$ of cold ATP the observed increase was gradually reduced: While the addition of seedling extract and 1 mM ATP still enhanced MBP-PIP5K3 activity about 5-fold, the incubation of MBP-PIP5K3 with seedling extract and 5 mM ATP increased activity only about 2-fold (Fig. 3.29). Seedling extract 
alone had no recognizable PI4P 5-kinase activity (data not shown). As another control MBPPIP5K3 was incubated without exogenous ATP and the activity was compared to the activity of MBP-PIP5K3 treated with 5 mM of ATP. As depicted in Fig. 3.29 the activity of ATP-treated and untreated MBP-PIP5K3 was similar suggesting that cold ATP alone was not able to reduce MBP-PIP5K3 activity or had an effect on the incorporation of $y-\left[{ }^{32} P\right] A T P$ in the subsequent activity test. Overall the treatment of MBP-PIP5K3 with the complex plant extract revealed both an ATP-dependent inhibition of PI4P 5-kinase activity and a massive unexpected activation not requiring ATP that is not sensitive to the presence of phosphatase inhibitors.

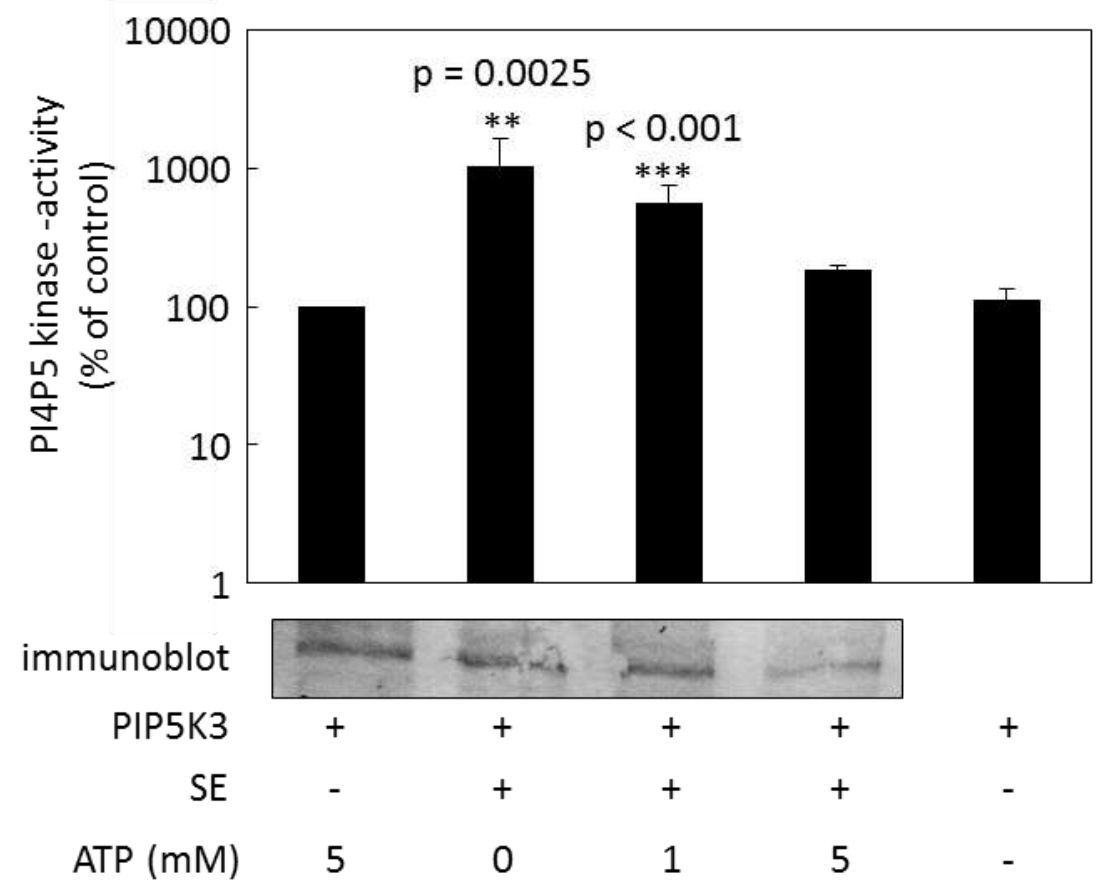

Fig. 3.29: Arabidopsis seedling extract modulates PIP5K3 activity. Equal amounts of purified MBPPIP5K3 were incubated with Arabidopsis seedling extract (SE) and 0-5 mM of cold ATP. Additionally MBP-PIP5K3 was treated without SE and ATP (right). The activity was tested by applying radiolabled $\gamma$ $\left[{ }^{32} \mathrm{P}\right]$ ATP and Ptdlns4P. Lipid products were extracted, analyzed by TLC and quantified by phosphoimaging. Immunoblots using an MBP-antibody were used as loading controls. Data show the PI4P 5 kinase activity of each sample in percent compared to a control incubated with buffer and $5 \mathrm{mM}$ of cold ATP (left). Please note the logarithmic scale. Data represent the means \pm SD of 3 independent experiments, each assayed in duplicates. The asterisks indicate a significant decrease of PI4P 5-kinase activity after incubation with SE and $5 \mathrm{mM}$ ATP compared to the buffer-treated control according to a student's t-test.

\subsection{Effects of PIP5K3 T472A/D mutations on catalytic activity}

To identify additional phosphorylation sites of PIP5K3 by MS-analysis, purified recombinant MBP-PIP5K3 was treated with seedling extract, digested with trypsin (see section 2.2.6) and the digested peptides were applied to mass spectrometry (Dr. Waltraud Schulze, Golm). Unfortunately, the analysed peptides only covered about $7.5 \%$ of the PIP5K3 sequence as 
shown in Figure 3.30 and no additional phosphorylation sites were identified. A peptide array experiment similar to that for PIP5K2 (cf. Figure 3.14) was not attempted and subsequent experiments on PIP5K3 therefore focused on the characterization of the confirmed site T472.

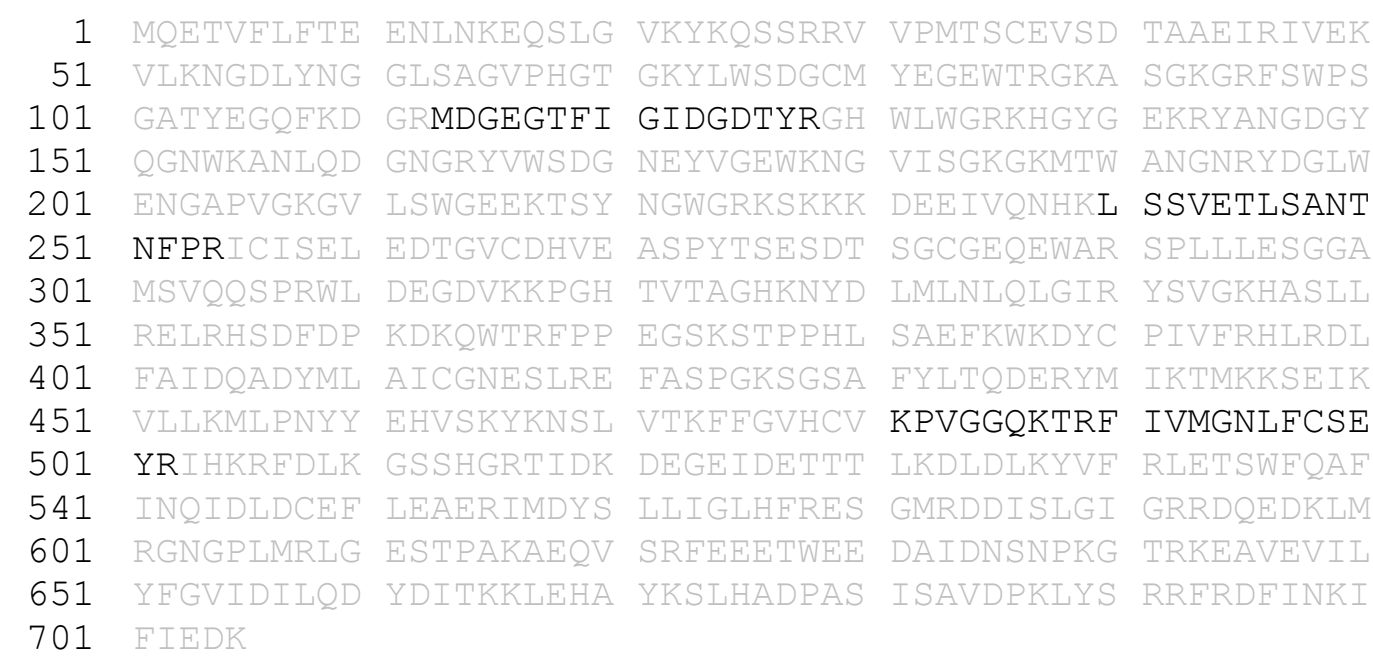

Fig. 3.30: Sequence coverage of PIP5K3 after MS-analysis of trypsin digested peptides. Purified recombinant MBP-PIP5K3 was prephosphorylated in vitro, digested with trypsin and the resulting peptides were analysed by mass spectrometry. Shown is the amino acid sequence of PIP5K3. Black, identified peptides. Grey, not accessible to MS.

The phosphorylation site found in PhosPhAT 4.0 at position PIP5K3 T472 is located in the catalytic domain and was chosen for further investigation by site directed mutagenesis. As already described in the material and methods section and in section 3.5 effects of phosphorylation were investigated by altering the selected threonine residue to $A$ (phosphoablation), and to $D$ (phosphomimicry). The resulting $A / D$ variants were heterologously expressed in E. coli Rosetta 2 cells and equal protein concentrations of crude extracts were tested for changes in PI4P 5-kinase activity by applying radiolabeled $\left.\gamma-{ }^{32} \mathrm{P}\right]$ ATP and Ptdlns4P as substrates. The activity of MBP-PIP5K3 T472 A/D variants was compared to that of a MBP-PIP5K3 wild type control and is visualized in Figure 3.31. As depicted, MBPPIP5K3 T472A was more active than wild type MBP-PIP5K3, whereas the corresponding D variant was nearly inactive. The investigated T472 A/D combination, thus, showed an activity pattern corresponding to the working hypothesis on the effects of phosphorylation on PI4P 5kinase activity according to Rao et al. (1998). 


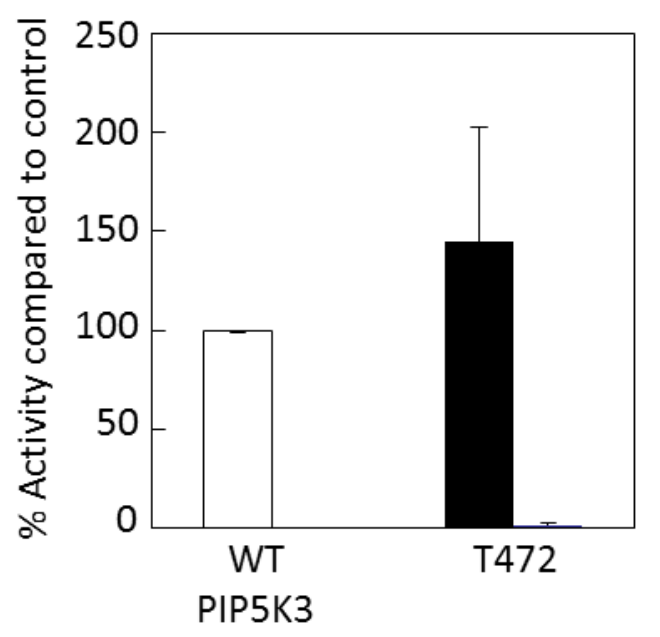

Fig. 3.31: PI4P 5-kinase activity of MBP-PIP5K3 T472 A/D variants. The experimentally verified phosphorylation site PIP5K3 T472 was altered to A and D and recombinantly expressed in E. coli. Equal protein amounts of crude extract were tested for catalytic activity by adding PtdIns4P and radiolabeled $\mathrm{Y}-\left[{ }^{32} \mathrm{P}\right]$ ATP. Lipids were extracted, separated by TLC and radiolabled Ptdlns $(4,5) \mathrm{P}_{2}$ was quantified by a phosphoimager. Graphic shows activity of A- (black) and D- (grey) variants compared to wild type (wt) PIP5K3 (white) and represents means of 2 experiments, \pm SD.

The degree of in vitro phosphorylation of MBP-PIP5K3 T472A was not further investigated, as has been shown for MBP-PIP52 T430 A and T500A (c.f. Fig. 3.17) because of the limited phosphorylation of wild type MBP-PIP5K3, which was barely detectable by the methods used.

\subsection{Subcellular localization of fluorescence tagged PIP5K3 T472A/D variants transiently expressed in tobacco pollen tubes}

Because of the clear results for catalytic activity in vitro, it was next tested whether phosphomimicry or phosphoablation of residue T472 exerted an effect on plasma membrane association of PIP5K3. PIP5K3 or the respective variants PIPK3 T472A/D were fused to an Nterminal EYFP and transiently expressed in tobacco pollen tubes. Plasma membrane association was investigated after $7 \mathrm{~h}$ of growth in pollen tubes showing vigorous cytoplasmic streaming and is depicted in Figure 3.32. As was previously described in the literature (Stenzel et al., 2008) EYFP-PIP5K3 displayed a distinct localization at the apical tip of the growing pollen tube (Fig. 3.32). Similarly, EYFP-PIPK3 T472A and EYFP-PIP5K3 T472D also localized predominantly to the plasma membrane (Fig. 3.32) in patterns not distinguishable from the distribution of the fluorescence-tagged wild type PIP5K3. 


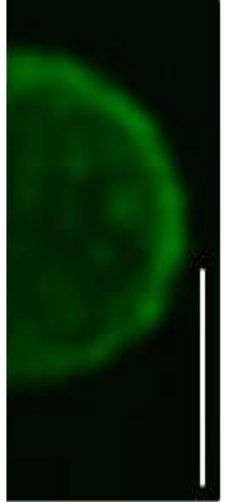

WT PIP5K3

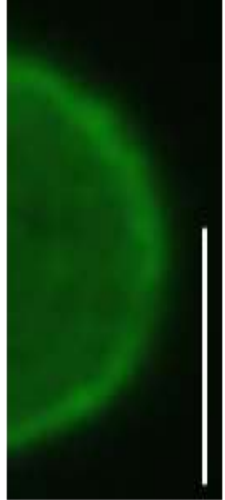

T472A

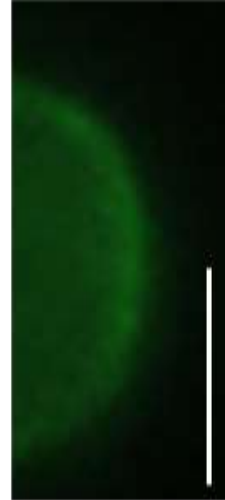

T472D

Fig. 3.32: Subcellular localization of PIP5K3-YFP wild type and corresponding A/D-combinations in tobacco pollen tubes. Tobacco pollen tubes were transformed with EYFP-PIP5K3 (wt) and EYFP-PIP5K3 T472A, and EYFP-PIP5K3 T472D. Pollen tubes were grown for $7 \mathrm{~h}$ and EYFP-PIP5K3 localization was imaged by epifluoreszence microscopy. Images are representative for 100 transformed pollen tubes per construct. Bars, $10 \mu \mathrm{m}$.

So far investigations on PIP5K3 indicate that position T472 is a possible phosphorylation site. Altering the respective amino acid to A or D had severe effects on PIP5K3 functionality but did not alter membrane association in growing tobacco pollen tubes. To test whether PIP5K3 T472A/D variants had an effect on in vivo functionality, PIP5K3 T472A and D variants were tested for their ability to complement an Arabidopsis pip5k3 T-DNA insertion mutant phenotype as will be described in the next section.

\subsection{Mutant complementation}

In previous experiments it was found that Arabidopsis PIP5K3 is essential for root hair formation. Arabidopsis mutant plants homozygous for T-DNA-disrupted PIP5K3 alleles were compromised in root hair formation (Stenzel et al., 2008). Therefore it was tested if stable expression of PIP5K3 T472A or PIP5K3 T472D were able to complement for he reduced root hair phenotype thus indicating in vivo functionality of the respective expressed protein variant(s).

PIP5K3 T472 A and PIP5K3 T472D CDNA was expressed in roots of the Arabidopsis pip5k3 mutant line pip5k3-4 under the control of a 1500-bp PIP5K3 promoter fragment as a fusion to an N-terminal EYFP tag. Vectors were provided by Dr. Irene Stenzel (MLU Halle-Wittenberg). Wild type Arabidopsis controls, and the respective mutant controls were grown side by side with the transgenics on solid agar plates for 1 week and analyzed for their ability to form root hairs. Root hair length was quantified with Image J and the amount of root hairs either $0-80$ or over $80 \mu \mathrm{m}$ in length was illustrated (Fig. $3.33 \mathrm{~F}$ ). As depicted in Figure 33, pip5k3-KO mutants 
exhibited reduced root hair growth (Fig. 3.33 A and F striped bar) compared to wild type plants (Fig. $3.33 \mathrm{~B}$ and $\mathrm{F}$ white bar). pip5k3 EYFP-PIP5K3 T472D plants were also inhibited in root hair growth (Fig. $3.33 \mathrm{C}$ and F grey bar) whereas the expression EYFP-PIP5K3 T472A in the pip5k3ko mutant background reestablished root hair growth and thus complemented the phenotype (Fig. $3.33 \mathrm{D}$ and $\mathrm{F}$ white bar). The presence of the EYFP-PIP5K3 T472D transgene in the pip5k3 mutant background displaying short root hairs was verified by PCR-based genotyping (Fig. 3.33 E) 

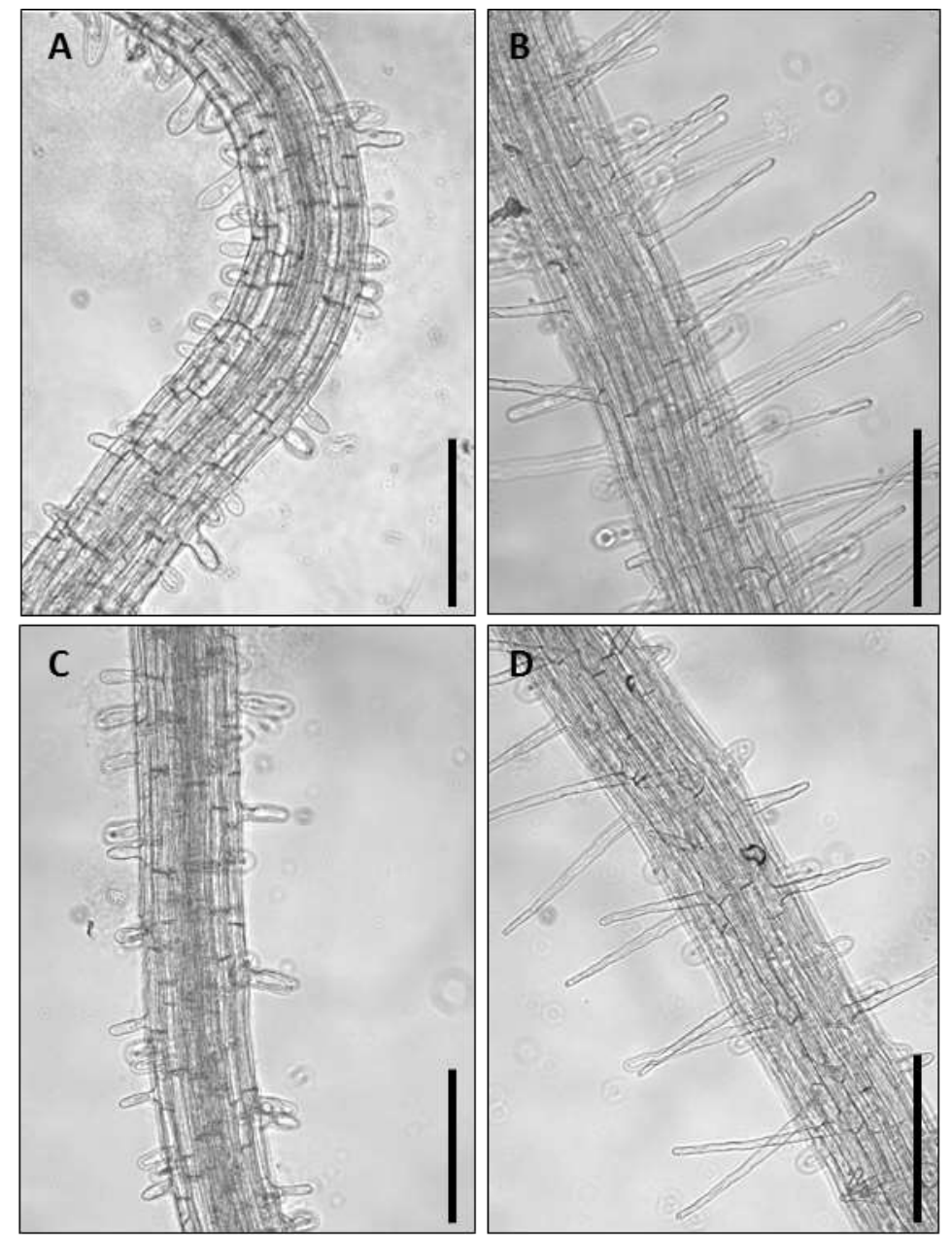

E

$F$
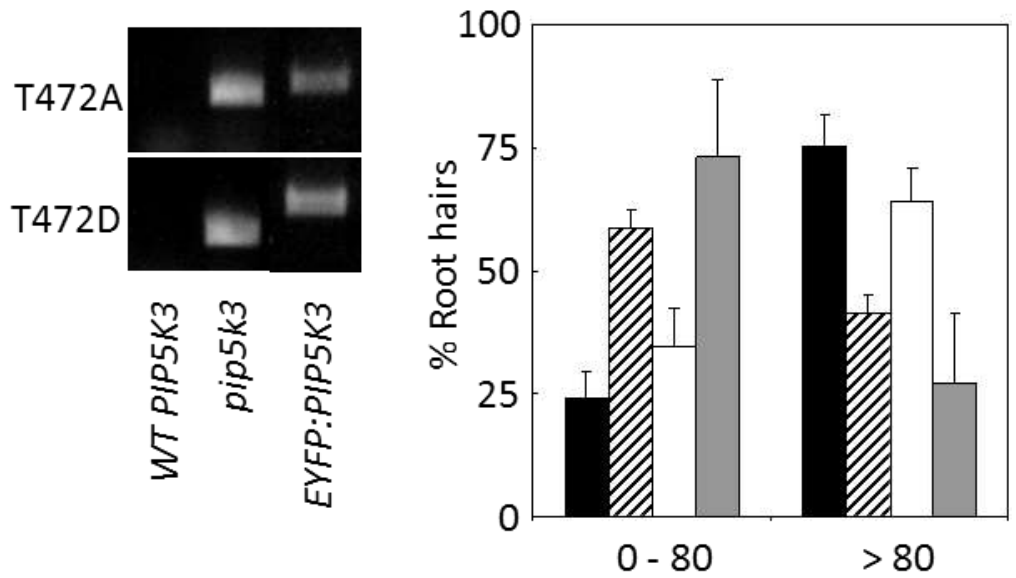

Root hair length $(\mu \mathrm{m})$

Fig. 3.33: Root hair morphology of wild type plants, pipk5k3 T-DNA insertion mutants and pip5k3 EYFP-PIP5K3 T472A/D mutants. pip5k3-ko mutants were transformed with PIP5K3::EYFP:PIP5K3 T472A and PIP5K3::EYFP:PIP5K3 T472D respectively. Plants were grown on agar plates for $7 \mathrm{~d}$ and digital images were taken. A, pip5k3 mutant. B, wild type. C, pip5k3 mutant expressing EYFP-PIP5K3 T472D. D, pip5k3 mutant expressing EYFP-PIP5K3 T472A. Bars $=200 \mu \mathrm{m}$. E, The presence of the EYFP-PIP5K3 transgene was verified by genotyping. F, Quantification of root hair length in wild type plants, pipk3mutants and variants. Striped bar, pip $5 k 3$ mutant. Black bar, wild type. White bar, pip5k3 EYFP-PIP5K3 T472D. Grey bar, pip5k3 EYFP-PIP5K3 T472A. Data represent 2 independent experiments with over 300 investigated root hairs. 


\section{Discussion}

Posttranslational modification by phosphorylation exerts important regulatory effects on cellular processes. In the context of this thesis the phosphoinositide kinases PIP5K2 and PIP5K3 were investigated for their ability to become phosphorylated by different plant extracts and it was established that phosphorylation resulted in the inhibition of catalytic activity. Phosphorylation sites of PIP5K2 and PIP5K3 were identified, the respective residues altered to mimic the dephosphorylated or the phosphorylated state of the enzyme and the enzyme variants characterized regarding in vitro activity, in vivo localization and in vivo functionality.

\subsection{Some technical aspects of expression and enrichment of recombinant MBP PIP5K2 and MBP-PIP5K3 in E. coli}

In previous studies (Stenzel et al., 2008; Ischebeck et al., 2008; Stenzel et al., 2012), recombinant expression of PI4P 5-kinases from A. thaliana proved difficult and recombinant protein was not generated in the substantial amounts required for detailed biochemical characterization. To enable the characterization of in vitro phosphorylation of Arabidopsis PI4P 5-kinases, the first aim of this thesis was to optimize heterologous expression of recombinant MBP-PIP5K2 and MBP-PIP5K3 in E. coli. Recombinant MBP-PIP5K2 and MBP-PIP5K3 were enriched by affinity chromatography via the MBP-tag. As illustrated in Fig 3.1 and 3.24 pronounced full length bands for MBP-PIP5K2 and MBP-PIP5K3 were detectable after enrichment that had not been detectable in corresponding crude extracts. The expression of the enzymes as fusions to the large N-terminal MBP-tags furthermore facilitated solubility of the recombinant proteins. In addition to full length proteins, however, additional smaller bands were visible in the Coomassie-stained SDS-gels and were also detectable in immunoblots, indicating that these proteins represented smaller protein fragments of MBPPIP5K2 and MBP-PIP5K3, likely originating from premature termination events during translation of the recombinant fusion proteins. It is important to note that the presence of the smaller PI4P 5-kinase fragments impairs the accurate estimation of protein concentrations, resulting in the underestimation of specific catalytic activities after protein enrichment (cf. Table 3.1 and Table 3.4). While the fusion of PIP5K2 or PIP5K3 to N-terminal MBP-tags, thus, represents a disadvantage for the recombinant expression in $E$. coli, it nonetheless enabled 
substantial levels of expression of full-length proteins. Importantly, the technical limitations mentioned do not impair the overall interpretation of the biochemical characterization presented.

To test whether the enriched MBP-PIPK2 and MBP-PIP5K3 proteins were still active after purification in vitro activity tests with radiolabeled ATP and Ptdlns $(4,5) \mathrm{P}_{2}$ as substrate were conducted. By comparing the specific activity of crude extracts with that of the corresponding enriched fractions, a purification factor for both PI4P 5-kinases was calculated (Table 3.1 and 3.4). After affinity chromatography, recombinant MBP-PIP5K2 was at least 5-fold enriched compared to crude extracts. In contrast, enriched MBP-PIP5K3 lost specific activity compared to crude extract. Because both the Coomassie-stained SDS-gel and the immunoblot of affinityenriched MBP-PIP5K3 showed a more concentrated band for full-length MBP-PIP5K3 than was observed in crude extract (Fig. 3.1 and Fig. 3.24), it is likely that MBP-PIP5K3 specific activity decreased during purification. Although it is known that PI4P 5-kinase activity is dependent on the presence of $\mathrm{MgCl}_{2}$ molecules it is possible that the $E$. coli crude extract contains intrinsic cofactors or ions that are necessary for full catalytic activation of the PIP5K3 enzyme. During purification, the MBP-PIP5K3 binds to the MBP-trap matrix, whereas salts and cofactors are collected in the exclusion volume and are not available for the enzyme anymore, thus, decreasing the catalytic activity. As described above, it is also possible that the reduced specific activity of enriched MBP-PIP5K3 is a consequence of the presence of catalytically inactive $\mathrm{C}$-terminally truncated protein fragments arising from cotranslational termination that distort protein estimates and result in an underestimation of the specific catalytic activity of the recombinant full-length protein.

\subsection{Similarities and differences of in vitro phosphorylation of MBP-PIP5K2 and MBP-PIP5K3}

Reversible phosphorylation of recombinant Arabidopsis PIP5K2 and PIP5K3 was investigated in vitro by applying radiolabeled ATP in the presence of PKA or plant extracts exhibiting protein kinase activity. Although full-length PIP5K2 and PIP5K3 were shown to get reversibly phosphorylated by PKA (Fig. 3.3 and 3.26) and by the relevant plant extracts (Fig. 3.4 and 3.27), there were severe differences in the phosphorylation status of both enzymes. The in vitro phosphorylation assays with PKA indicated that the extent of ${ }^{32} \mathrm{P}$-incorporation in recombinant PIP5K2 was higher than that detected for PIP5K3 (Fig. 3.3 and 3.26), suggesting that PKA might prefer PIP5K2 as a substrate. Differences in the technical procedure can be excluded, since PIP5K2 and PIP5K3 were incubated side-by-side with the same amount of PKA and 
radiolabeled ATP under identical conditions, and phosphoimages were exposed for identical times. In line with the notion that PKA might have a higher affinity towards PIP5K2 than to PIP5K3 is the observation that peptide array analyses with PKA revealed about 8 possible phosphorylation sites for PIP5K2 (section 3.4.4) of which only 4 are conserved in PIP5K3 (S274, S275, S370, S455). Additionally, research of information on NetPhosK 2.0 (http://www.cbs.dtu.dk/services/ NetPhosK/; (Blom et al., 1999), a database predicting kinase specific eukaryotic protein phosphorylation sites, predicted 10 putative phosphorylation sites recognized by PKA for PIP5K2, whereas only 8 putative phosphorylation sites of PIP5K3 were predicted to be recognized by PKA. These data support the notion that PIP5K2 contains more sequence motives that are specifically phosphorylated by PKA compared to PIP5K3.

Since PKA is a protein kinase not present in plants, recombinant PIP5K2 and PIP5K3 were also incubated with soluble extracts of Arabidopsis plants and investigated for their phosphorylation status in vitro. Arabidopsis extract of 6-weeks-old plants phosphorylated both full-length MBP-PIP5K2 and full-length MBP-PIP5K3 (Fig. 3.4 and 3.27); however again PIP5K2 was phosphorylated to a higher extent than PIP5K3. A possible conclusion is that PIP5K3 contains fewer sequence motifs specifically recognized by protein kinases present in the plant extract compared to PIP5K2, as has also been concluded for differences in the phosphorylation status of PIP5K2 and PIP5K3 after incubation with PKA. Still, it should be noticed that the leaf extract is a highly complex protein mixture possibly containing protein kinases, phosphatases, proteases and even more protein classes dynamically interacting with each other. Therefore, the data might enable different routes of interpretation. Furthermore, protein kinases are tightly controlled since they are key activators or inhibitors of a diverse set of cellular processes and often only active when phosphorylated themselves. The observation that PIP5K3 was less phosphorylated by a plant extract compared to PIP5K2 can therefore have different explanations. For instance, it is possible, that protein kinase/s specifically phosphorylating PIP5K3 was/were not active or were even degraded, although the experimental setup included a potent mixture of inhibitors of phosphatases and proteases. As already described in the material and methods section the ratio between cold ATP and radiolabeled ATP included in the sample mixture is very important for the functionality of protein kinases. Therefore, it is also possible that the concentration of cold ATP did not approximate the $\mathrm{Km}$ value of the protein kinase/s phosphorylating PIP5K3, and in consequence the phosphorylation reaction might have proceeded slower on MBP-PIP5K3 than on MBPPIP5K2. Another possibility is that cofactors like $\mathrm{Mg}^{2+}$ or $\mathrm{Ca}^{2+}$ needed for full activation of a particular protein kinase were not present in sufficient concentrations. 
Protein kinases have important functions in the mediation of developmental and environmental signals and it is likely that the complement and/or activity status of protein kinases differs between plant organs or during the various stages of plant growth. Since PIP5K3 is predominantly expressed in roots and in seedlings, it was tested whether Arabidopsis seedling extract was capable of phosphorylating MBP-PIP5K3 to a higher extent than leaf extract prepared from 6-weeks-old plants. As illustrated in Figure 3.26 no increase in the extent of phosphorylation of MBP-PIP5K3 treated with seedling extract was detectable compared to leaf extract-treated MBP-PIP5K3 (please note the different amounts of MBPPIP5K3 protein in the Coomassie-stained SDS-gel shown in Fig $3.27 \mathrm{~A}$ ). It was observed that radiolabeled MBP-PIP5K3 phosphorylated by the leaf extract migrated higher than PIP5K3 treated with seedling extract. The observed size difference suggests an additional modification of the MBP-PIP5K3 molecule, the nature of which is so far unknown. It is possible that MBPPIP5K3 was subject to acetylation, glycosylation and/or myristoylation, resulting in the altered migration of the recombinant protein in the SDS-PAGE. This observation leaves room for future investigations.

\subsection{In vitro phosphorylation decreases catalytic activity of MBP-PIP5K2 and MBP- PIP5K3}

Based on the concept presented by Rao et al. (Rao et al., 1998) (Section 1.6) it was a working hypothesis of this study that the introduction of negatively charged groups, such as phosphateresidues, into the tertiary structure of a PI4P 5-kinase results in changes in the catalytic activity of the enzyme. To test this hypothesis, recombinant MBP-PIP5K2 and MBP-PIP5K3 were incubated with PKA or with plant extracts, respectively, followed by in vitro activity tests.

After incubation of MBP-PIP5K2 or MBP-PIP5K3 with PKA and $5 \mathrm{mM}$ ATP, the catalytic activities of either enzyme were reduced by about $40-50 \%$ (Fig. 3.6 and Fig. 3.26). This reduction is consistent with previous reports from other model systems that phosphorylation of PI4P 5kinases results in decreased catalytic activity (Vancurova et al., 1999; Park et al., 2001; Westergren et al., 2001). The decrease of MBP-PIPK3 activity after incubation with PKA was quite unexpected, since in phosphorylation assays only very limited phosphorylation of MBPPIP5K3 had been detected (Fig. 3.26). Although the extent of ${ }^{32} \mathrm{P}$-incorporation into MBPPIP5K2 was stronger than in PIP5K3 after PKA treatment (Fig. 3.3 and Fig. 3.26), catalytic activity of phosphorylated MBP-PIP5K2 was similarly decreased. Therefore, it appears possible that regulation of PIP5K2 activity involves the phosphorylation of more than one site. 
Because PKA does not naturally occur in plants, it was tested whether plant-endogenous protein kinase activity would also phosphorylate recombinant MBP-PIP5K2 or MBP-PIP5K3 in vitro. Treatment of PIP5K2 with leaf extract and $5 \mathrm{mM}$ ATP decreased PI4P 5-kinase activity about $60 \%$ (Fig. 3.7), resembling the observations from PKA-treatment (Fig. 3.6). SAP applied to counteract phosphorylation of MBP-PIP5K2 was not able to restore catalytic activity. While this observation might suggest that the effects described so far were not due to protein phosphorylation, the failure to dephosphorylate is as likely a consequence of the presence of potent phosphatase inhibitors included in the assay mixture. The notion that phosphorylation is indeed responsible for the effects shown in Figures 3.6 and 3.7 is further supported by the observation that the decrease in catalytic activity of MBP-PIP5K2 was ATP dependent and incubation of MBP-PIP5K2 with leaf extract in the absence of exogenous ATP or with only $1 \mathrm{mM}$ ATP did not change catalytic activity. The possibility that leaf extract-induced degradation of MBP-PIP5K2 was responsible for the detected decrease in catalytic activity was excluded by immunoblot analysis (Fig. 3.7 B). The data suggest that incubation of MBP-PIP5K2 with the extract resulted in its phosphorylation, inhibiting catalytic activity in vitro.

In contrast to the inhibition of MBP-PIP5K2 upon incubation with leaf extract, incubation of MBP-PIP5K3 with seedling extract resulted in a 10-fold increase of catalytic activity (Fig. 3.29). The increase was independent from ATP and not sensitive to phosphatase inhibitors included in the reaction mix, suggesting that the enhanced catalytic activity was not related to phosphorylation of MBP-PIP5K3. It is possible that the complex Arabidopsis seedling extract contains endogenous cofactors or ions that are needed for full catalytic activity of PIP5K3 and that are also present in $E$. coli crude extracts but got washed out during purification. This notion might also be an additional explanation for the low specific activity initially determined for recombinant MBP-PIP5K3 enriched by affinity-chromatography (Table 3.4), which could obviously be reactivated (Fig. 3.29). Another possibility is activation of MBP-PIP5K3 by a protein partner present in the seedling extract, the binding to which enhances activity. A possible future experiment to confirm this would be the treatment of PIP5K3 with a denaturated seedling extract and measuring the ability to form $\operatorname{Ptd} \ln s(4,5) \mathrm{P}_{2}$. Interestingly, the application of increasing levels of ATP reduced the enhanced activity stepwise until PI4P 5kinase activity reached the catalytic activity of buffer-treated PIP5K3 again. The data indicate a) that MBP-PIP5K3 activity is enhanced by an unknown component, cofactor or protein ligand present in seedling extract, and b) that the thus activated form of MBP-PIP5K3 is inhibited in its activity by a component of the seedling extract in an ATP dependent fashion and likely by phosphorylation. 


\subsection{N-terminal regions of PIP5K2 might be important for its regulation}

In previous studies it was suggested that N-terminal domains of plant PI4P 5-kinases are involved in the regulation of catalytic activity (Im et al., 2007). While the plant-specific $\mathrm{N}$ terminal extensions of PI4P 5-kinases from Arabidopsis are not required for catalytic activity (Im et al., 2007), (Stenzel et al., 2008); (Ischebeck et al., 2008), it has been proposed that they act as autoinhibitory elements based on the observation that PI4P 5-kinase variants lacking the $\mathrm{N}$-terminal domains display enhanced specific activities (Im et al., 2007), including variants of PIP5K2 (Stenzel et al., 2012) and PIP5K3 (Stenzel et al., 2008). Moreover, it has been demonstrated that an $\mathrm{N}$-terminal domain of PIP5K2 is required for correct subcellular localization and might direct PIP5K2 towards protein-protein interactions required for its specific regulatory context in vivo (Stenzel et al., 2012). To test whether N-terminal domains of a plant PI4P 5-kinase exerted an influence on the phosphorylation status of PIP5K2 by an Arabidopsis leaf extract, several truncated variants of PIP5K2 were created, incubated with leaf extract and $\mathrm{p}-\left[{ }^{32} \mathrm{P}\right] \mathrm{ATP}$ and then analyzed for the extent of ${ }^{32} \mathrm{P}$ incorporation as well as for their catalytic activity. The combined observations that PIP5K2 variants lacking the extreme $\mathrm{N}$ terminus and MORN domain were phosphorylated to a reduced degree compared to that of PIP5K2 full length protein (Fig. 3.10) and that the catalytic activity of this truncation was not influenced as severely by the incubation with leaf extract as the full-length enzyme (Fig. 3.11) indicate the presence of phosphorylation sites in the extreme $\mathrm{N}$-terminus and the MORNdomain that might be important for the regulation of catalytic activity. It is possible that phosphorylation of these domains directly inhibits catalytic activity by modulating the interaction with protein partners, possibly including the catalytic region of PIP5K2, or the binding to substrate vesicles. The additional removal of the Lin-domain increased the phosphorylation status again to wild type level (Fig. 3.10) with no changes in catalytic activity compared to MBP- $\triangle N T-M O R N$ after incubation with leaf extract (Fig. 3.11). It is possible that the elimination of the Lin-domain rendered additional phosphorylation sites in the rest of the protein accessible that would otherwise not be exposed to the cytosol and thus not available for phosphorylation by a soluble protein kinase. The catalytic domain alone still displayed $50 \%$ phosphorylation compared to the wild type control, indicating that this domain might also hold phosphorylation sites. 


\subsection{Reliability of identified phosphorylation sites}

For the identification of phosphorylation sites in recombinant PIP5K2 and PIP5K3 different approaches were pursued. One of the approaches was the review of MS-based phosphopeptide data obtained by other groups that is collected in the database PhosPhat 4.0 (Heazlewood et al., 2008) and is freely available on the internet. In the PhosPhAt 4.0 database an experimentally verified phosphorylation site was found for PIP5K2 at position S409. For PIP5K3 a phosphorylation site at position T472 was revealed, that is conserved in PIP5K2 at position T500. NetPhos prediction software (Blom et al., 1999) was used to determine further putative phosphorylation sites in PIP5K2. As already indicated in the material and methods section specific amino acid sequence motives preferably recognized by protein kinases have been identified for various eukaryotic organisms, but only little is known about such recognition motives in plants. The NetPhos software (Blom et al., 1999) predicted several conserved serine, threonine and tyrosine residues for PIP5K2 and the positions S370, S475, S541 and T546 were chosen for further investigations based on this prediction.

The identification of sites specifically phosphorylated by an Arabidopsis leaf extract was performed by MS analysis. Although the identification of peptides via MS is a high throughput method, the identification of post translational modifications is difficult because modifications, such as phosphorylation, only occur on a small amount of peptides (Kersten et al., 2009). To improve the detection of lowly abundant phosphorylation sites high sequence coverage is required, meaning that the identified peptides should represent a mostly complete protein sequence. The sequence coverage itself depends on the length of the peptide fragments generated after digestion, the purity of the sample and the sensitivity of the detection. If the peptide fragments are too short it is possible that their signal is lost in the background. Other limitations of MS analysis are contaminations with Keratins or trypsin autolysis that increase the background, as well as non-specific cleavage or missed cleavage of proteins that directly impede the identification of protein fragments. For PIP5K2 it was possible to identify two phosphorylation sites by MS, T430 and S598. These phosphorylation sites are both located in the catalytic domain of the protein. Although phosphorylation sites were predicted also for the $\mathrm{N}$-terminal domains, as described in the previous section, no sites were detected by MS in these domains despite of very good sequence coverage in the MS-analysis (Fig. 3.13). It is possible that phosphorylation sites in the $\mathrm{N}$-terminal domains were not detected because of their low abundance or unfavorable ionization properties of the respective tryptic phosphopeptides. 
No additional phosphorylation site was detected for PIP5K3 in our MS analyses. In contrast to the very good sequence coverage for MBP-PIP5K2 (Fig. 3.13), coverage for MBP-PIP5K3 was substantially inferior (Fig. 3.30). The low sequence coverage of MBP-PIP5K3 might in part explain the failure to detect more phosphorylation sites in the protein. Because the extent of MBP-PIP5K3 phosphorylation (Fig. 3.27) was overall lower than that of MBP-PIP5K2 (Fig. 3.4), it is also possible that phosphorylated fragments were present in concentrations too low to be detected with any confidence by MS.

In a last attempt to identify phosphorylation sites in PIP5K2 peptide arrays were used. In a first round of experiments peptide arrays were incubated with commercially available PKA and radiolabeled ATP (Fig. 3.14). Overall 16 PIP5K2 peptides were phosphorylated by PKA on the peptide array with different intensities, suggesting that peptides with particularly high phosphorylation intensity contained more than one phosphorylation site or were bound with a higher affinity. In contrast to incubation with highly concentrated commercial PKA, incubation of peptide arrays with leaf extract gave no meaningful results (Fig. 3.14). Although it has been shown in previous experiments that MBP-PIP5K2 can be phosphorylated by Arabidopsis leaf extract, it should be noted that the peptide array represents an artificial system of peptides bound onto a glass surface. It is possible that other proteins in the extract were also able to bind to the peptides and prevented putative protein kinases from phosphorylating. An additional problem is the adhesion of protein kinases to matrix surfaces (Thiele et al., 2010)that might result in phosphorylation too low to detect.

While it was verified that PI4P 5-kinases can be phosphorylated in vitro, a notion already proposed by others (Vancurova et al., 1999; Park et al., 2001) and in particular for PI4P 5kinases from Arabidopsis (Westergren et al., 2001), experiments in this thesis extend our current understanding by identifying several new phosphorylation sites in the Arabidopsis PI4P 5-kinase isoforms PIP5K2 and PIP5K3 (Fig. 3.13 and section 3.16).

\subsection{Phosphomimicry of some identified phosphorylation sites inhibits catalytic activity of PIP5K2 and PIP5K3}

To further characterize the phosphorylation sites identified, residues were altered to A or D, the resulting MBP-PIP5K2 and MBP-PIP5K3 variants were recombinantly expressed in E. coli and tested for their activity in vitro. MBP-PIP5K2 T430A/D and MBP-PIP5K3 T472A/D represented activity-patterns supporting the working hypothesis for the effects of phosphorylation on catalytic activity, where the A-variants were as active as the wild type enzyme and the D-variants were less active (Fig. 3.15). These sites were experimentally verified 
and are strong candidates for phosphorylation sites regulating PI4P 5-kinase activity. For MBPPIP5K2 T500A/D the same activity pattern was observed, however here the activity of the A variant increased about $50 \%$ compared to wild type MBP-PIP5K2, whereas MBP-PIP5K2 T500D was catalytically inactive (Fig. 3.15). This site was chosen based on its sequence homology to phosphorylated PIP5K3 T472 and is consistent with predicted effects of phosphorylation on activity. Other substitutions tested based on positive MS-identification of phosphorylation sites, such as PIP5K2 S409A/D and S598A/D, were overall strongly reduced in catalytic activity, suggesting that the substitution of these sites had a negative effect on the folding of the PIP5K2 protein (Fig. 3.15). These sites were disregarded in further investigations. The phosphorylation sites that were altered to $A$ and $D$ based on computer aided prediction (PIP5K2 S370, S541 and T546) also supported the hypothesis (Fig. 3.16). The A variants were always more active than the corresponding $D$ variants, but reached only $30-50 \%$ of the activity of wild type PIP5K2. Therefore, these variants were not used in further experiments. As the PIP5K2 variants substituted in position S475 displayed equal activity regardless of whether this position was altered to $A$ or to $D$, this site was also not considered for further experiments (Fig. 3.16).

\subsection{Phosphoablation of position T430 renders the MBP-PIP5K2 protein less susceptible to phosphorylation by Arabidopsis leaf extract}

Based on the hypothesis that the A variant of a particular site prevents its phosphorylation, thus mimicking the constitutively dephosphorylated state of the enzyme, this variant should no longer be targeted by protein kinases. In previous experiments it was shown that MBPPIP5K2 T430A and T500A displayed high in vitro activity whereas the corresponding D variants were less active or even inactive (Fig. 3.15). Therefore it was tested whether the corresponding phosphoablated A-variants were still a substrate for in vitro phosphorylation by Arabidopsis leaf extract. As illustrated in Figure 3.17 the extent of ${ }^{32} \mathrm{P}$ incorporation into MBP-PIP5K2 T430A was reduced by about $50 \%$, indicating that this site indeed is a substrate for a protein kinase. Additionally the data indicate the presence of additional phosphorylation sites. In contrast to these observations, the incorporation of ${ }^{32} \mathrm{P}$ into MBP-PIP5K2 T500A was not impaired, indicating that this site is not a target for phosphorylation by the extracts used. 


\subsection{Are effects of phosphoablation and phosphomimicry related to altered in vivo localization of PIP5K2 and PIP5K3?}

As described in the introduction, the interaction between PI4P 5-kinase and lipid membrane is of key importance for substrate conversion. The association of fluorescence-tagged PIP5K2 and PIP5K3 variants to lipid membranes was analyzed in vivo. While the working hypothesis of this thesis suggested that phosphorylation of PI4P 5-kinases results in their dissociation from the membrane, the data obtained from the in vivo localization experiments (Fig. 3.18 and Fig. 3.32) indicate that membrane association of the investigated substituted variants of PIP5K2-EYFP or EYFP-PIP5K3 was not different to the localization of wild type PIP5K2-EYFP or wild type EYFPPIP5K3. These data indicate that the phosphorylation sites identified in previous experiments did not control membrane association. Additionally, the data also indicate that the selected protein variants are still able to fold correctly since EYFP alone did not localize to the membrane and displayed a diffuse cytosolic localization. The observations are in line with the previous report that PIP5K2 associates more tightly with the plasma membrane of pollen tube cells than some other isoforms of PI4P 5-kinases (Stenzel et al., 2012). In the previous study, the deletion of $\mathrm{N}$-terminal domains of PIP5K2 had not resulted in its dissociation from the membrane (Stenzel et al., 2012), as had been observed for some other isoforms (Stenzel et al., 2012; Ischebeck et al., 2008). The data suggest that the introduction of negative charges by phosphorylation or by phosphomimicry might alter the membrane association of PIP5K2-EYFP and EYFP-PIP5K3 in a more subtle fashion, possibly disabling access of the substrate Ptdlns4P to the catalytic centers while maintaining close association of the enzymes with the membrane (cf. Fig. 1.6).

\subsection{Phosphorylation of PIP5K2 and PIP5K3 in key positions is relevant for in vivo functionality.}

As described in section 3.9 the heterologous expression of Arabidopsis PIP5K2 in tobacco pollen tubes exerts characteristic effects on pollen tube morphology and can thus indicate in vivo functionality of the selected variants. Heterologous expression of either PIP5K2 T430AEYFP or PIP5K2 T430D-EYFP increased the amount of swollen pollen tubes, a known phenomenon for the synthesis of PIP5K2-derived Ptdlns(4,5) $P_{2}$ (Fig. 3.20). The data are consistent with results from the in vitro activity tests, where both MBP-PIP5K2 T430 A and MBP-PIP5K2 T430D displayed substantial catalytic activity (Fig. 3.15). Similarly, the expression of PIP5K2 T500A-EYFP also resulted in a high proportion of swollen pollen tube tips, whereas in 
contrast, pollen tubes expressing PIP5K2 T500D-EYFP displayed normal phenotypes. These data are also in line with the results from in vitro activity tests where the PIP5K2 T500A-EYFP variant was fully active, while the corresponding $D$ variant was inactive. Additional to experiments performed with pollen tubes, PIP5K2-EYFP T500A or PIP5K2-EYFP T500D were also expressed in the pip5k1 pip5k2 double mutant background. Since Arabidopsis pip5k1 pip5k2 double mutants have a severe phenotype, rescue of the phenotype by expression of the PIP5K2 variants investigated so far can aid in the understanding of the role of phosphorylation in the physiological context. The expression of PIP5K2-EYFP T500A in the pip5k1 pip5k2 double mutant background was able to rescue the phenotype. Double mutant plants expressing PIP5K2-EYFP T500A grew normal, indicating that this particular PIP5K2 variant fully assumed physiological functions. For future experiments it would be interesting to test whether double mutant plants expressing PIP5K2-EYFP T500A still respond normally to stress treatment in the same way as has been shown for wild type plants. In contrast, expression of PIP5K2-EYFP T500D did not rescue the pip5k1 pip5k2 mutant phenotype, indicating a lack of function for that PIP5K2 variant. The data are in line with data observed from in vitro activity tests and heterologous expression in tobacco pollen tubes (Fig. 3.15 and Fig. 3.20).

Since PIP5K3 is not expressed in pollen (Stenzel et al., 2008) and has no recognizable effect on the morphology of tobacco pollen tubes upon heterologous expression, PIP5K3-EYFP T472A and PIP5K3-EYFP T472D were not tested for in vivo functionality in pollen. However, the availability of an Arabidopsis T-DNA insertion mutant with a clear phenotype enabled the analysis of in vivo functionality of ectopically expressed variant forms of PIP5K3-EYFP by testing for their ability to rescue the phenotype of the pip $5 k 3$ mutant. The Arabidopsis pip $5 k 3$ mutant is compromised in root hair formation (Stenzel et al., 2008). As depicted in Figure 3.31 the expression of PIP5K3-EYFP T472A in the pip5k3 mutant background was able to reestablish root hair growth, whereas expression of PIP5K3-EYFP T472D did not complement the phenotype. These data are consistent with the corresponding in vitro activity tests of the respective PIP5K3-variants and indicate that the phosphorylation site T472 is a regulator of catalytic function not only in vitro but also in vivo.

\subsection{The hypothesis of Rao et al. (1998) has to be expanded}

The model proposed by Rao et al. (1998) suggests that one mode by which phosphorylation of PI4P 5-kinases might influence their catalytic function is by introducing negative charges at the flattened interfacial region facing the membrane (Rao et al., 1998). The interface region 
contains many positively charged residues, facilitating interaction with membranes rich in anionic phospholipids, such as Ptdlns4P, the substrate of PI4P 5-kinases. The introduction of negative charges into the membrane interface region might, thus, impair membrane association, interfere with membrane-association of the enzyme and, decrease catalytic activity (Burden et al., 1999). Aided by the crystal structure of the human PIPKII $\beta$ (Rao et al., 1998), a 3D-model of the plant PI4P 5-kinase, PIP5K2, was developed (Fig. 4.1-4.3 ; Ingo Heilmann, personal communication). Importantly, the model of Arabidopsis PIP5K2 displays a pronounced accumulation of positive surface charges at the proposed membrane interface region (Fig. 4.1-4.3), similar to the pattern found for human PIPKIIß (Fig. 1.5).

In this thesis it was shown that treatment of recombinant MBP-PIP5K2 and of MBP-PIP5K3 with PKA or with complex biological extracts resulted in phosphorylation of the proteins. When the positions of verified and putative phosphorylation sites investigated in this study were mapped onto the 3D-model of Arabidopsis PIP5K2 (Fig. 4.1-4.3), most of the sites were not located at the membrane interface region. For instance, only one putative phosphorylation site suggested for PIP5K2 by computer-aided prediction (S475, Fig. 4.3) was located in the membrane interface region rich in positive charges, whereas none of the MBP-PIP5K2 residues experimentally determined to be phosphorylated by PKA or leaf extracts were located in that region. The only phosphorylation site found for PIP5K3 in the PhosphAt-database was also not located in the membrane interface region of PIP5K3. These surprising findings indicate that phosphorylation might control PI4P 5-kinases in a manner different from that proposed by Rao and coworkers (Rao et al., 1998). In this context it is important to note that phosphomimicry and phosphoablation nonetheless exerted substantial effects on in vitro catalytic activity (Fig. 3.15, Fig. 3.16 and Fig. 3.31), and on in vivo functionality of the enzymes (Fig. 3.20, Fig. 3.22 and Fig 3.33), albeit without influencing the membrane association of the respective variants of PIP5K2-EYFP or EYFP-PIP5K3 (Fig. 3.18 and Fig. 3.32). It is likely that changes in PI4P 5-kinase surface charges that are introduced by phosphorylation or phosphomimicry result in conformational changes modulating catalytic activity. Such changes can influence the geometry of key residues in the catalytic sites and/or influence the affinity for substrate binding by enabling or preventing access of substrate lipids to the catalytic sites. 


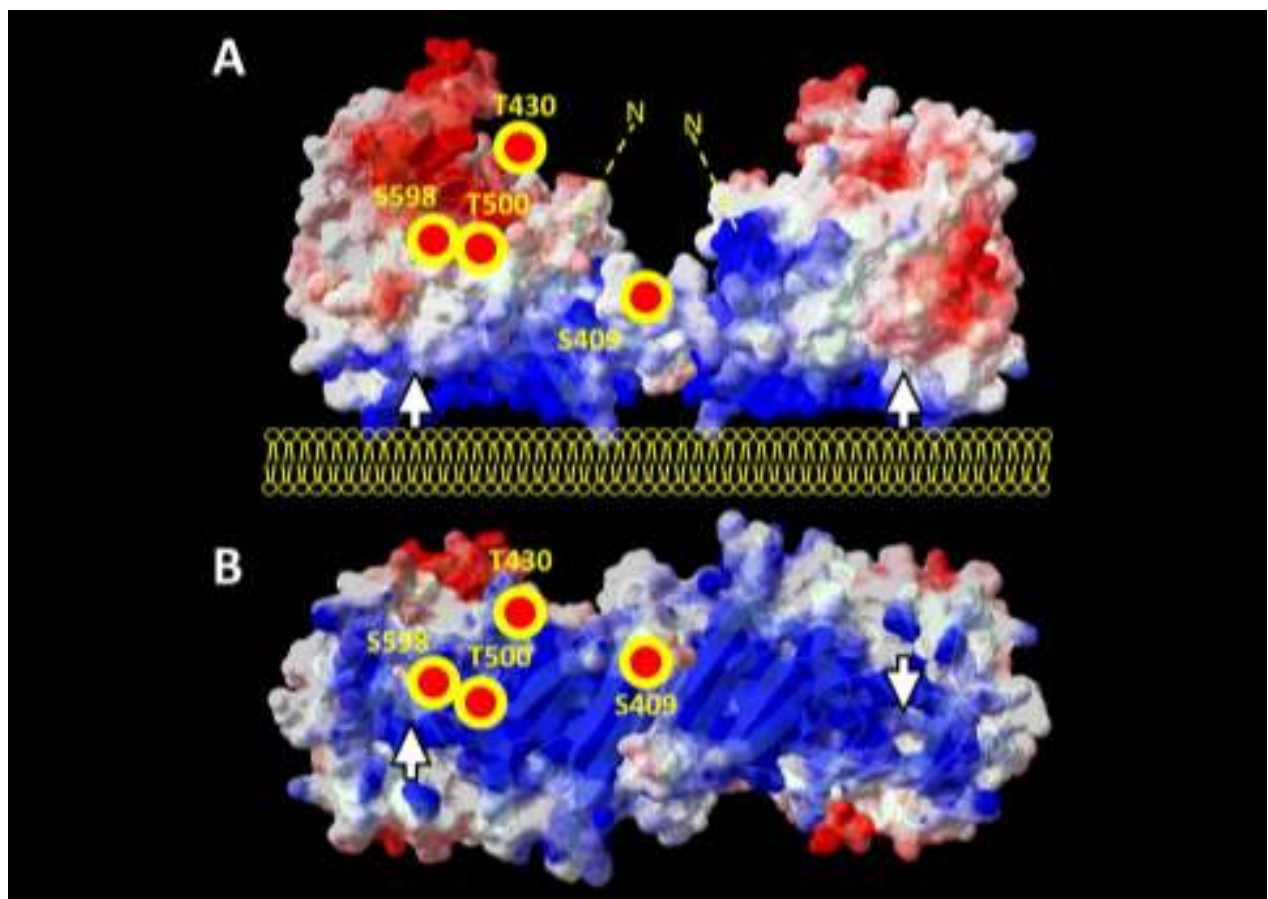

Fig. 4.1. Residues in Arabidopsis PIP5K2 that are strong phosphorylation candidates. Position S409, T430 and S598 are phosphorylation sites that were experimentally verified upon treatment with plant extracts. T500 was chosen based on sequence homology to a phosphorylation site found for PIP5K3 at position T472. The electrostatic potential is indicated by the colors and was calculated by GRASP (Graphical Representation And Analysis of Structural Proteins; (Honig and Nicholls, 1995)). Red, Negative charges. Blue, Positive charges. A, Association of Arabidopsis PIP5K2 with the model membrane consisting (yellow). B, Dimer in ventral orientation. The catalytic centers are indicated with arrows.

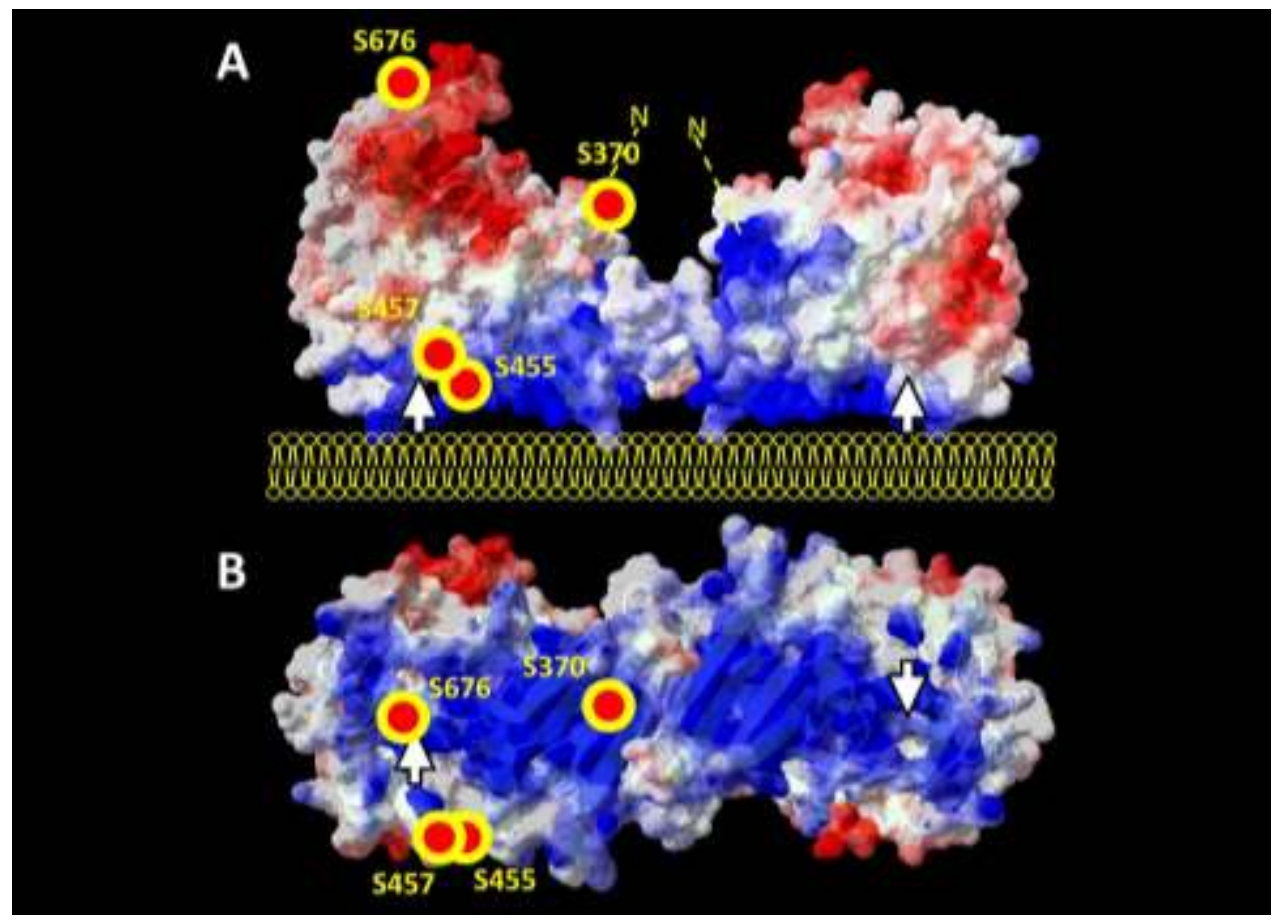

Fig. 4.2. Phosphorylation sites in Arabidopsis PIP5K2 experimentally verified upon PKA treatment. Shown are the residues located to the cytosolic side and the membrane facing site of PIP5K2. The electrostatic potential is indicated by the colors and was calculated by GRASP (Graphical Representation And Analysis of Structural Proteins; (Honig and Nicholls, 1995)). Red, Negative charges. Blue, Positive charges. A, Association of Arabidopsis PIP5K2 with the model membrane consisting (yellow). B, Dimer in ventral orientation. The catalytic centers are indicated with arrows. 


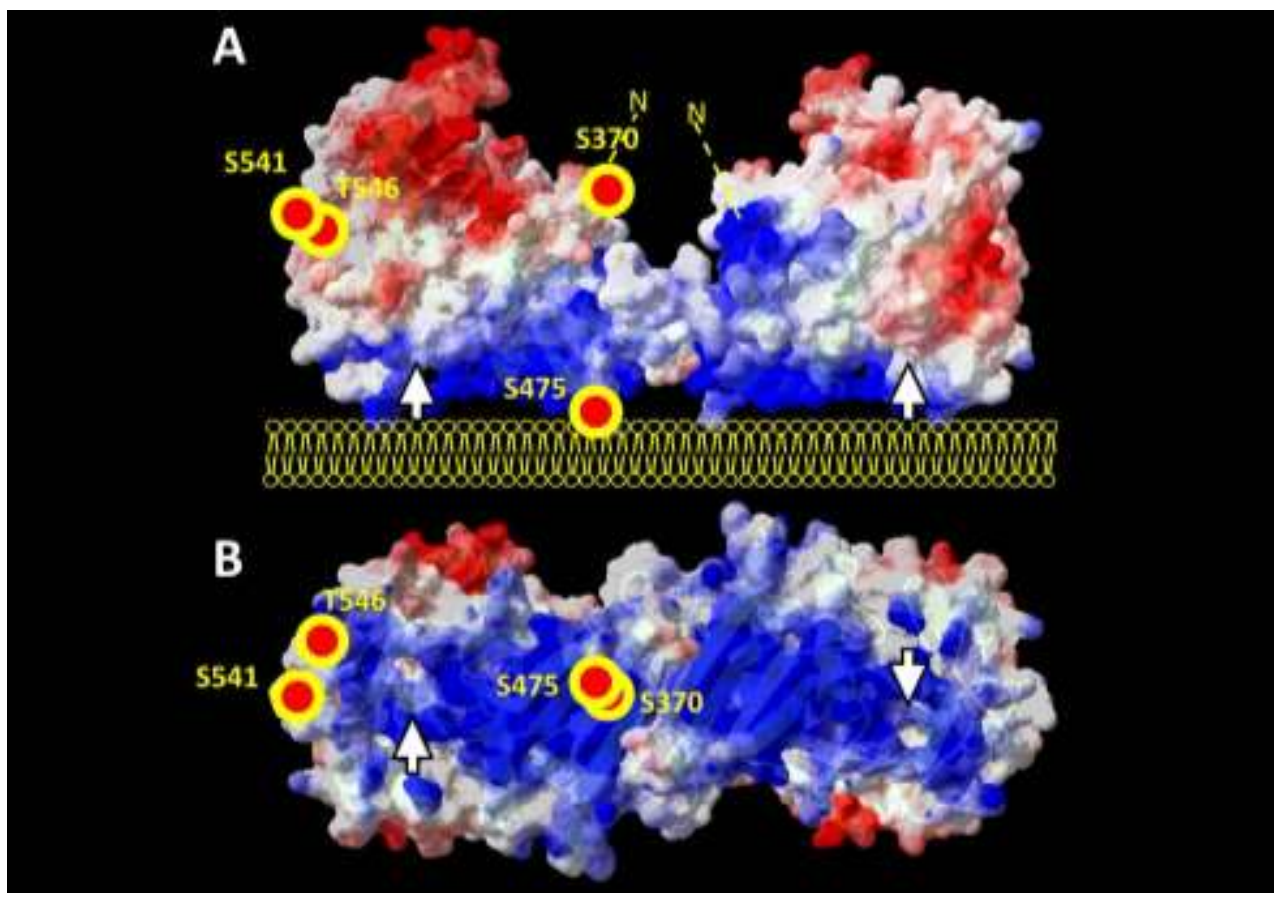

Fig. 4.2. Putative phosphorylation sites in Arabidopsis PIP5K2 based on computer aided prediction. Residues were chosen based on their high phosphorylation score predicted by NetPhos 2.0 and their high conservation in other members of the PIP5K2 family. The electrostatic potential is indicated by the colors and was calculated by GRASP (Graphical Representation And Analysis of Structural Proteins; (Honig and Nicholls, 1995)). Red, Negative charges. Blue, Positive charges. A, Association of Arabidopsis PIP5K2 with the model membrane consisting (yellow). B, Dimer in ventral orientation. The catalytic centers are indicated with arrows.

The data suggest several categories of verified or putative phosphorylation sites of PIP5K2 according to their accessibility for soluble protein kinases at the protein surface and their effects of phosphomimicry or phosphoablation on the catalytic activity of MBP-PIP5K2 variants. The first category includes sites that - according to the 3D-model (Fig. 4.1 - Fig. 4.3) are exposed at the surface of the PI4P 5-kinase protein and that display clearly different effects on catalytic activity upon phosphomimicry and phosphoablation. Sites in this category are T430 or S370. Experiments on the in vivo functionality of PIP5K2 T430A-EYFP and PIP5K2 T430 D-EYFP (Fig. 3.20 and Fig. 3.22) are consistent with the determined in vitro activities for these variants (Fig. 3.15), indicating T430 as a prime candidate for a site with regulatory effects on the function of PIP5K2.

The second category includes residues that are exposed, but whose substitution has either no effect on activity or abolishes activity regardless of what amino acid is introduced. Sites in this category include S409 and S475. The relevance of phosphorylation sites of category two is unclear. It is possible that phosphorylation of such sites might effect a conformational change of the PIP5K2 protein as a prerequisite for further modifications, possibly exposing or masking additional sites of posttranslational modifications. 
A third category represents sites that are not exposed at the surface of the PI4P 5-kinase protein, but are located within the catalytic sites. Examples for the third category are T500 or S598 of PIP5K2 and T472 of PIP5K3. Interestingly, phosphomimicry and phosphoablation of some of these sites, such as T472 of PIP5K3, results in abolished and unimpaired catalytic activity, respectively, suggesting a regulatory role of the charge-distribution around the particular site even within the catalytic center. Position S598 is located immediately adjacent to the ATP binding pocket of PIP5K2. Substitution of this site had severe effects on catalytic activity, and it is possible that a mutation to alanine or aspartate blocks ATP binding or even the transfer of the phosphate group to the inositol group of the substrate lipid. Based on the location of the phosphorylation sites of category three, it appears unlikely that a soluble protein kinase can access these sites, and they are candidates for autophosphorylation. The notion that T472 of PIP5K3 is autophosphorylated is consistent with the observation of very low phosphorylation of MBP-PIP5K3 by PKA or biological extracts. While regulation of PIP5K3 by autophosphorylation might be an interesting concept to pursue in future experiments, it should be highlighted that the 3D-model, based on which T500 and T598 of PIP5K2 or T472 of PIP5K3 are proposed to reside within the catalytic site, might be flawed and should be further optimized, and the analysis redone.

The observation that no phosphorylation of MBP-PIP5K2 or MBP-PIP5K3 occurred at the membrane interface region under the conditions used raises the question whether the experimental design of the in vitro phosphorylation tests might prevent access of this particular region for soluble protein kinases. It is possible that electrostatic interactions of the basic membrane interface region of PI4P 5-kinases with anionic components of the extract or with detergents present in the assay mix might occupy this region, masking putative phosphorylation sites and rendering them inaccessible to in vitro-phosphorylation. While this notion cannot be disregarded for MBP-fusions treated with complex biological extracts, there was also no phosphorylation upon treatment with purified recombinant PKA, which would not introduce many anionic components into the reaction mixture. Overall, it cannot be excluded that phosphorylation sites at the membrane interface region of PIP5K2 or PIP5K3 have been missed in the experiments presented in this thesis. Thus, the hypothesis of Rao et al. (1998) was not rejected for plant PI4P 5-kinases. However, the positive identification of several additional phosphorylation sites that are likely not located at the membrane interface suggests that factors beyond membrane association play a role in the control of PI4P 5-kinases by phosphorylation. Such factors include possible conformational changes in the PI4P 5-kinase protein upon phosphorylation of exposed residues at the cytosolic surface of the protein as well as possibly autophosphorylation of residues within the catalytic sites of the lipid kinase. 


\subsection{PI4P 5-kinases and their role in the response of plants to environmental stimuli}

As already described in the introduction, cellular PtdIns $(4,5) \mathrm{P}_{2}$-levels increase rapidly upon exposing plants to hyperosmotic stress (Heilmann et al., 1999; Pical et al., 1999; König et al., 2007), suggesting a fast activation of the PI4P 5-kinases relevant for the stress induced production of PtdIns $(4,5) P_{2}$. PIP5K2 is a prime candidate for PI4P 5-kinases with roles in the response to hyperosmotic stress, because it is ubiquitously expressed in all organs of Arabidopsis. Treatment of PIP5K2 with extracts prepared from stressed plants had no adverse effect on the phosphorylation status of PIP5K2 (Fig. 3.23), indicating that the activity of protein kinases phosphorylating PIP5K2 was not changed in these extracts. Based on the information that phosphorylation of PI4P 5-kinases inhibits their activity, it follows that an activation of the enzymes might be achieved by their dephosphorylation. As described in the Material and Methods section, the preparation of plants extracts as required for this study must include phosphatase inhibitors, and therefore the action of phosphatases cannot be analyzed under the conditions used.

The data presented in this thesis indicate phosphorylation by plant-endogenous enzymes as one of the posttranslational modifications impeding PI4P 5-kinase activity. The notion that phosphorylation inhibits PI4P 5-kinases raises the question for the relevance of such a mode of regulation at a larger scale. The emerging picture suggests that under normal conditions PI4P 5-kinases are present in an inactive, phosphorylated state, and the Ptdlns $(4,5) \mathrm{P}_{2}$-levels are low. Upon perception of stress PI4P 5-kinases can rapidly be activated by dephosphorylation, enabling increases of Ptdlns $(4,5) \mathrm{P}_{2}$-levels. In this fashion the plant can keep a functional signalling system in an inactive state during periods of favorable conditions. Once the environment turns unfavourable and stress is perceived, the activation of the phosphorylated PI4P 5-kinases will not cost additional energy that is possibly not available anymore due to the now limiting conditions. Based on the results presented here, it will be interesting to test whether a pre-phosphorylated MBP-PIP5K2 is dephosphorylated more rapidly by extracts prepared from plants challenged with hyperosmotic stress than by extracts from non-stressed plants. Overall, the data reported in this thesis contribute an important piece of information on the control of PI4P 5-kinase activity by posttranslational modification and will aid further insights into the adaption of plants to environmental stresses. 


\section{SUMMARY}

Phosphatidylinositol-4,5-bisphosphate $\left(\operatorname{Ptd} \ln (4,5) \mathrm{P}_{2}\right)$ is a phospholipid that controls a diverse set of physiological functions by modulating localization or activity of target proteins. Ptdlns $(4,5) \mathrm{P}_{2}$ is formed by PI4P 5-kinases, which occur as 11 isoenzymes in Arabidopsis. In various eukaryotic models PI4P 5-kinases are regulated by posttranslational modification and it has been shown that different PI4P 5-kinases from other model systems, including Arabidopsis PIP5K1, can be phosphorylated. Based on the solved X-ray structure of the human PIPKII $\beta$ it has been proposed that phosphorylation at the membrane-oriented face of the protein impairs electrostatical interactions with the substrate membrane and thus, inhibits catalytic activity. For plant PI4P 5-kinases only little information is available and so far it remained unclear whether other Arabidopsis PI4P 5-kinases can be phosphorylated, what amino acids are phosphorylated, and what consequences arise for localization and functionality of the enzymes in plants. In the context of this thesis, phosphorylation of two Arabidopsis PI4P 5kinases, PIP5K2 and PIP5K3 was investigated. Recombinantly expressed and affinity-purified PIP5K2 and PIP5K3 were found to be phosphorylated in vitro by a commercially available PKA and also by complex Arabidopsis extracts containing protein kinase activity. Phosphorylation of PIP5K2 and PIP5K3 decreased catalytic activity in an ATP dependent manner. Interestingly, treatment of PIP5K3 with plant extract without added ATP increased activity, whereas the addition of ATP resulted in a decrease in activity, indicating the presence of both activating and inhibiting factors in the plant extract. The sites of phosphorylation were determined by computer aided prediction, MS-based analysis of tryptic peptides and by peptide array analysis, and several phosphorylation sites were determined for PIP5K2 and PIP5K3. The respective amino acids were changed to alanine or aspartate residues, mimicking the dephosphorylated or phosphorylated states, respectively. The recombinant variant proteins were characterized in vitro for catalytic function and their subcellular localization was analysed during heterologous expression as fluorescence-tagged fusions in tobacco pollen tubes. Variant proteins were also expressed in relevant Arabidopsis mutant backgrounds and tested for complementation of known phenotypes. The investigations revealed PIP5K2 T430, PIP5K2 T500 and PIP5K3 T472 as strong candidates for phosphorylation sites. The location of the phosphorylation sites on a 3D-model of plant PI4P 5-kinases indicates that the control of PI4P 5-kinases by phosphorylation not only occurs by controlling membrane association but also includes phosphorylation sites not located in the protein-membrane interface. 


\section{LITERATURE}

Alberts, B., Johnson, A., Lewis, J., Raff, M., Roberts, K., and Walter, P. (2002). Molecular Biology of the Cell. (New York: Garland Science).

Anderson, R.A., Boronenkov, I.V., Doughman, S.D., Kunz, J., and Loijens, J.C. (1999). Phosphatidylinositol phosphate kinases, a multifaceted family of signaling enzymes. J Biol Chem 274, 9907-9910.

Audhya, A., and Emr, S.D. (2003). Regulation of PI4,5P2 synthesis by nuclear-cytoplasmic shuttling of the Mss4 lipid kinase. Embo J 22, 4223-4236.

Bailly, M., Ichetovkin, I., Grant, W., Zebda, N., Machesky, L.M., Segall, J.E., and Condeelis, J. (2001). The F-actin side binding activity of the Arp2/3 complex is essential for actin nucleation and lamellipod extension. Curr Biol 11, 620-625.

Balla, T. (2005). Inositol-lipid binding motifs: signal integrators through protein-lipid and protein-protein interactions. J Cell Sci 118, 2093-2104.

Balla, T. (2006). Phosphoinositide-derived messengers in endocrine signaling. J Endocrinol 188, 135-153.

Benzioni, A., and Forti, M. (1989). Jojoba. In Oil Crops of the World, G. Robbelen, R.K. Downey, and A. Ashri, eds (New York: McGraw-Hill Publishing Company), pp. 448-461.

Berdy, S.E., Kudla, J., Gruissem, W., and Gillaspy, G.E. (2001). Molecular characterization of At5PTase1, an inositol phosphatase capable of terminating inositol trisphosphate signaling. Plant Physiol 126, 801-810.

Berridge, M.J. (1983). Rapid accumulation of inositol trisphosphate reveals that agonists hydrolyse polyphosphoinositides instead of phosphatidylinositol. Biochem J 212, 849858.

Berridge, M.J., and Irvine, R.F. (1984). Inositol trisphosphate, a novel second messenger in cellular signal transduction. Nature 312, 315-321.

Blom, N., Gammeltoft, S., and Brunak, S. (1999). Sequence and structure-based prediction of eukaryotic protein phosphorylation sites. Journal of molecular biology 294, 1351-1362.

Bradford, M.M. (1976). A rapid and sensitive method for the quantitation of microgram quantities of protein utilizing the principle of protein-dye binding. Anal Biochem 72, 248-254.

Braun, M., Baluska, F., von Witsch, M., and Menzel, D. (1999). Redistribution of actin, profilin and phosphatidylinositol-4, 5-bisphosphate in growing and maturing root hairs. Planta 209, 435-443.

Burden, L.M., Rao, V.D., Murray, D., Ghirlando, R., Doughman, S.D., Anderson, R.A., and Hurley, J.H. (1999). The flattened face of type II beta phosphatidylinositol phosphate kinase binds acidic phospholipid membranes. Biochemistry 38, 15141-15149.

Carricaburu, V., and Fournier, B. (2001). Phosphoinositide fatty acids regulate phosphatidylinositol 5-kinase, phospholipase $\mathrm{C}$ and protein kinase $\mathrm{C}$ activities. European journal of biochemistry / FEBS 268, 1238-1249.

Chen, Q., Brglez, I., and Boss, W.F. (1991). Inositol phospholipids as plant second messengers. Symp Soc Exp Biol 45, 159-175.

Cho, M.H., and Boss, W.F. (1995). Transmembrane signaling and phosphoinositides. Methods Cell Biol 49, 543-554.

Cho, M.H., Chen, Q., Okpodu, C.M., and Boss, W.F. (1992). Separation and quantification of of $\left[{ }^{3} \mathrm{H}\right]$ inositol phospholipids using thin-layer-chromatography and a computerized ${ }^{3} \mathrm{H}$ imaging scanner. LC-GC 10, 464-468. 
Ciruela, A., Hinchliffe, K.A., Divecha, N., and Irvine, R.F. (2000). Nuclear targeting of the beta isoform of type II phosphatidylinositol phosphate kinase (phosphatidylinositol 5phosphate 4-kinase) by its alpha-helix 7. Biochem J 346 Pt 3, 587-591.

Clarke, J.H., Richardson, J.P., Hinchliffe, K.A., and Irvine, R.F. (2007). Type II PtdInsP kinases: location, regulation and function. Biochem Soc Symp, 149-159.

Clough, S.J., and Bent, A.F. (1998). Floral dip: a simplified method for Agrobacterium-mediated transformation of Arabidopsis thaliana. Plant J 16, 735-743.

Davis, A.J., Im, Y.J., Dubin, J.S., Tomer, K.B., and Boss, W.F. (2007). Arabidopsis phosphatidylinositol phosphate kinase 1 binds F-actin and recruits phosphatidylinositol 4-kinase beta1 to the actin cytoskeleton. J Biol Chem 282, 14121-14131.

de la Fuente van Bentem, S., Anrather, D., Roitinger, E., Djamei, A., Hufnagl, T., Barta, A., Csaszar, E., Dohnal, I., Lecourieux, D., and Hirt, H. (2006). Phosphoproteomics reveals extensive in vivo phosphorylation of Arabidopsis proteins involved in RNA metabolism. Nucleic Acids Res 34, 3267-3278.

Delmas, P., and Brown, D.A. (2005). Pathways modulating neural KCNQ/M (Kv7) potassium channels. Nat Rev Neurosci 6, 850-862.

Desrivieres, S., Cooke, F.T., Parker, P.J., and Hall, M.N. (1998). MSS4, a phosphatidylinositol-4phosphate 5-kinase required for organization of the actin cytoskeleton in Saccharomyces cerevisiae. J Biol Chem 273, 15787-15793.

DeWald, D.B., Torabinejad, J., Jones, C.A., Shope, J.C., Cangelosi, A.R., Thompson, J.E., Prestwich, G.D., and Hama, H. (2001). Rapid accumulation of phosphatidylinositol 4,5bisphosphate and inositol 1,4,5-trisphosphate correlates with calcium mobilization in salt-stressed Arabidopsis. Plant Physiol 126, 759-769.

Dissmeyer, N., and Schnittger, A. (2011). Use of phospho-site substitutions to analyze the biological relevance of phosphorylation events in regulatory networks. Methods Mol Biol 779, 93-138.

Doughman, R.L., Firestone, A.J., and Anderson, R.A. (2003). Phosphatidylinositol phosphate kinases put PI4,5P(2) in its place. J Membr Biol 194, 77-89.

Dowd, P.E., Coursol, S., Skirpan, A.L., Kao, T.H., and Gilroy, S. (2006). Petunia phospholipase c1 is involved in pollen tube growth. Plant Cell 18, 1438-1453.

Drobak, B.K., Dewey, R.E., and Boss, W.F. (1999). Phosphoinositide kinases and the synthesis of polyphosphoinositides in higher plant cells. Int Rev Cytol 189, 95-130.

Drobak, B.K., Watkins, P.A.C., Valenta, R., Dove, S.K., Lloyd, C.W., and Staiger, C.J. (1994). Inhibition of plant plasma membrane phosphoinositide phospholipase $\mathrm{C}$ by the actinbinding protein, profilin. Plant J 6, 389-400.

Elge, S., Brearley, C., Xia, H.J., Kehr, J., Xue, H.W., and Mueller-Roeber, B. (2001). An Arabidopsis inositol phospholipid kinase strongly expressed in procambial cells: synthesis of Ptdlns(4,5)P2 and Ptdlns(3,4,5)P3 in insect cells by 5-phosphorylation of precursors. Plant J 26, 561-571.

Endemann, G., Dunn, S.N., and Cantley, L.C. (1987). Bovine brain contains two types of phosphatidylinositol kinase. Biochemistry 26, 6845-6852.

Engelman DM (2005) Membranes are more mosaic than fluid. Nature 438: 578-580

Engelsberger, W. R., Schulze, W. X. (2011). Nitrate and ammonium lead to distinct global dynamic phosphorylation patterns when resupplied to nitrogen-starved Arabidopsis seedlings. Plant J 69, 978-995

Ercetin, M.E., and Gillaspy, G.E. (2004). Molecular characterization of an Arabidopsis gene encoding a phospholipid-specific inositol polyphosphate 5-phosphatase. Plant Physiol 135, 938-946.

Estacion, M., Sinkins, W.G., and Schilling, W.P. (2001). Regulation of Drosophila transient receptor potential-like (TrpL) channels by phospholipase C-dependent mechanisms. J Physiol 530, 1-19. 
Foti, M., Audhya, A., and Emr, S.D. (2001). Sac1 lipid phosphatase and Stt4 phosphatidylinositol 4-kinase regulate a pool of phosphatidylinositol 4-phosphate that functions in the control of the actin cytoskeleton and vacuole morphology. Mol Biol Cell 12, 2396-2411.

Gilroy, S., Read, N.D., and Trewavas, A.J. (1990). Elevation of cytoplasmic calcium by caged calcium or caged inositol triphosphate initiates stomatal closure. Nature 346, 769-771.

Graves, P.R., and Haystead, T.A. (2003). A functional proteomics approach to signal transduction. Recent Prog Horm Res 58, 1-24.

Gungabissoon, R.A., Jiang, C.-J., Drobak, B.K., Maciver, S.K., and Hussey, P.J. (1998). Interaction of maize actin-depolymerising factor with actin and phosphoinositides and its inhibition of plant phospholipase C. Plant J 16, 689-696.

Gupta, R., Ting, J.T., Sokolov, L.N., Johnson, S.A., and Luan, S. (2002). A tumor suppressor homolog, AtPTEN1, is essential for pollen development in Arabidopsis. Plant Cell 14, 2495-2507.

Haider, S., Tarasov, A.I., Craig, T.J., Sansom, M.S., and Ashcroft, F.M. (2007). Identification of the PIP2-binding site on Kir6.2 by molecular modelling and functional analysis. EMBO J 26, 3749-3759.

Heazlewood, J.L., Durek, P., Hummel, J., Selbig, J., Weckwerth, W., Walther, D., and Schulze, W.X. (2008). PhosPhAt: a database of phosphorylation sites in Arabidopsis thaliana and a plant-specific phosphorylation site predictor. Nucleic Acids Res 36, D1015-1021.

Heilmann, I. (2008). Towards understanding the function of stress-inducible Ptdlns $(4,5) \mathrm{P}_{2}$ in plants. Communicative \& Integrative Biology 1, 204-206.

Heilmann, I. (2009). Using genetic tools to understand plant phosphoinositide signalling. Trends Plant Sci 14, 171-179.

Heilmann, I., Perera, I.Y., Gross, W., and Boss, W.F. (1999). Changes in phosphoinositide metabolism with days in culture affect signal transduction pathways in Galdieria sulphuraria. Plant Physiol 119, 1331-1339.

Heilmann, I., Perera, I.Y., Gross, W., and Boss, W.F. (2001). Plasma membrane phosphatidylinositol 4,5-bisphosphate levels decrease with time in culture. Plant Physiol 126, 1507-1518.

Hetherington, A.M. (2001). Guard cell signaling. Cell 107, 711-714.

Hilgemann, D.W., and Ball, R. (1996). Regulation of cardiac Na+,Ca2+ exchange and KATP potassium channels by PIP2. Science 273, 956-959.

Hilpela, P., Vartiainen, M.K., and Lappalainen, P. (2004). Regulation of the actin cytoskeleton by $\mathrm{PI}(4,5) \mathrm{P} 2$ and $\mathrm{PI}(3,4,5) \mathrm{P} 3$. Curr Top Microbiol Immunol 282, 117-163.

Hinchliffe, K.A., Ciruela, A., and Irvine, R.F. (1998). PIPkins1, their substrates and their products: new functions for old enzymes. Biochim Biophys Acta 1436, 87-104.

Hofgen, R., and Willmitzer, L. (1988). Storage of competent cells for Agrobacterium transformation. Nucleic Acids Res 16, 9877.

Honig, B., and Nicholls, A. (1995). Classical electrostatics in biology and chemistry. Science 268, 1144-1149.

Hood, E.E., Gelvin, S.B., Melchers, L.S., and Hoekema, A. (1993). New Agrobacterium helper plasmids for gene transfer to plants. Transgenic Res 2, 208-218.

Horton, H.R., Moran, L.A., Scrimgeour, K.G., Perry, M.D., and Rawn, J.D. (2006). Lipids and membranes. In Principles of Biochemistry, G. Carlson, ed (Upper Saddle River, New Jersey: Pearson Prentice Hall), pp. 253-292.

Huang, H.J., Lin, Y.M., Huang, D.D., Takahashi, T., and Sugiyama, M. (2003). Protein tyrosine phosphorylation during phytohormone-stimulated cell proliferation in Arabidopsis Hypocotyls. Plant Cell Physiol 44, 770-775.

Hughes, W.E., Cooke, F.T., and Parker, P.J. (2000). Sac phosphatase domain proteins. Biochem J 350, 337-352. 
Hunter, T. (2007). The age of crosstalk: phosphorylation, ubiquitination, and beyond. Molecular cell 28, 730-738.

Hunter, T. (2009). Tyrosine phosphorylation: thirty years and counting. Curr Opin Cell Biol 21, 140-146.

Hurley, J.H., and Grobler, J.A. (1997). Protein kinase C and phospholipase C: bilayer interactions and regulation. Curr Opin Struct Biol 7, 557-565.

Im, Y.J., Davis, A.J., Perera, I.Y., Johannes, E., Allen, N.S., and Boss, W.F. (2007). The Nterminal Membrane Occupation and Recognition Nexus Domain of Arabidopsis Phosphatidylinositol Phosphate Kinase 1 Regulates Enzyme Activity. J Biol Chem 282, 5443-5452.

Inoue H, Nojima H, Okayama H (1990) High efficiency transformation of Escherichia coli with plasmids. Gene 96: 23-28

Ischebeck, T., and Heilmann, I. (2010). PIP-kinases as key regulators of plant function. In Lipid Signaling in Plants, T. Munnik, ed (Berlin, Germany: Springer), pp. 79-93.

Ischebeck, T., Stenzel, I., and Heilmann, I. (2008). Type B phosphatidylinositol-4-phosphate 5kinases mediate pollen tube growth in Nicotiana tabacum and Arabidopsis by regulating apical pectin secretion. Plant Cell 20, 3312-3330.

Ischebeck, T., Seiler, S., and Heilmann, I. (2010). At the poles across kingdoms: phosphoinositides and polar tip growth. Protoplasma 240, 13-31.

Ischebeck, T., Stenzel, I., Hempel, F., Jin, X., Mosblech, A., and Heilmann, I. (2011). Phosphatidylinositol-4,5-bisphosphate influences $\mathrm{Nt}-\mathrm{Rac5}$-mediated cell expansion in pollen tubes of Nicotiana tabacum. Plant J 65, 453-468.

Jackson, M., Crick, D.C., and Brennan, P.J. (2000). Phosphatidylinositol is an essential phospholipid of mycobacteria. J Biol Chem 275, 30092-30099.

Janke, C., Magiera, M.M., Rathfelder, N., Taxis, C., Reber, S., Maekawa, H., Moreno-Borchart, A., Doenges, G., Schwob, E., Schiebel, E., and Knop, M. (2004). A versatile toolbox for PCR-based tagging of yeast genes: new fluorescent proteins, more markers and promoter substitution cassettes. Yeast 21, 947-962.

Justin, A.M., Hmyene, A., Kader, J.C., and Mazliak, P. (1995). Compared selectivities of the phosphatidylinositol-synthase from maize coleoptiles either in microsomal membranes or after solubilization. Biochim Biophys Acta 1255, 161-166.

Kersten, B., Agrawal, G.K., Durek, P., Neigenfind, J., Schulze, W., Walther, D., and Rakwal, R. (2009). Plant phosphoproteomics: an update. Proteomics 9, 964-988.

King, C.E., Stephens, L.R., Hawkins, P.T., Guy, G.R., and Michell, R.H. (1987). Multiple metabolic pools of phosphoinositides and phosphatidate in human erythrocytes incubated in a medium that permits rapid transmembrane exchange of phosphate. Biochem J 244, 209-217.

König, S., Mosblech, A., and Heilmann, I. (2007). Stress-inducible and constitutive phosphoinositide pools have distinct fatty acid patterns in Arabidopsis thaliana. FASEB J 21, 1958-1967.

König, S., Ischebeck, T., Lerche, J., Stenzel, I., and Heilmann, I. (2008). Salt stress-induced association of phosphatidylinositol-4,5-bisphosphate with clathrin-coated vesicles in plants. Biochem J 415, 387-399.

Kost, B. (2008). Spatial control of Rho (Rac-Rop) signaling in tip-growing plant cells. Trends Cell Biol 18, 119-127.

Kost, B., Lemichez, E., Spielhofer, P., Hong, Y., Tolias, K., Carpenter, C., and Chua, N.H. (1999). Rac homologues and compartmentalized phosphatidylinositol 4, 5-bisphosphate act in a common pathway to regulate polar pollen tube growth. J Cell Biol 145, 317-330.

Kovar, D.R., Drobak, B.K., and Staiger, C.J. (2000). Maize profilin isoforms are functionally distinct. Plant Cell 12, 583-598. 
Kusano, H., Testerink, C., Vermeer, J.E.M., Tsuge, T., Shimada, H., Oka, A., Munnik, T., and Aoyama, T. (2008). The Arabidopsis phosphatidylinositol phosphate 5-kinase PIP5K3 is a key regulator of root hair tip growth. Plant Cell 20, 367-380.

Laemmli, U.K. (1970). Cleavage of structural proteins during the assembly of the head of bacteriophage T4. Nature 227, 680-685.

Lassing, I., and Lindberg, U. (1985). Specific interaction between phosphatidylinositol 4,5bisphosphate and profilactin. Nature 314, 472-474.

Lee, M.C., and Miller, E.A. (2007). Molecular mechanisms of COPII vesicle formation. Semin Cell Dev Biol 18, 424-434.

Lee, Y., Choi, Y.B., Suh, S., Lee, J., Assmann, S.M., Joe, C.O., Kelleher, J.F., and Crain, R.C. (1996). Abscisic Acid-Induced Phosphoinositide Turnover in Guard Cell Protoplasts of Vicia faba. Plant Physiol 110, 987-996.

Lee, Y., Kim, Y.W., Jeon, B.W., Park, K.Y., Suh, S.J., Seo, J., Kwak, J.M., Martinoia, E., and Hwang, I. (2007). Phosphatidylinositol 4,5-bisphosphate is important for stomatal opening. Plant J 52, 803-816.

Lemmon, M.A. (2003). Phosphoinositide recognition domains. Traffic 4, 201-213.

Li, C., Schilmiller, A.L., Liu, G., Lee, G.I., Jayanty, S., Sageman, C., Vrebalov, J., Giovannoni, J.J., Yagi, K., Kobayashi, Y., and Howe, G.A. (2005). Role of beta-oxidation in jasmonate biosynthesis and systemic wound signaling in tomato. Plant Cell 17, 971-986.

Liu, K., Li, L., and Luan, S. (2005). An essential function of phosphatidylinositol phosphates in activation of plant shaker-type $\mathrm{K}^{+}$channels. Plant J 42, 433-443.

Liu, Y., Casey, L., and Pike, L.J. (1998). Compartmentalization of phosphatidylinositol 4,5bisphosphate in low-density membrane domains in the absence of caveolin. Biochem Biophys Res Commun 245, 684-690.

Löfke, C., Ischebeck, T., König, S., Freitag, S., and Heilmann, I. (2008). Alternative metabolic fates of phosphatidylinositol produced by PI-synthase isoforms in Arabidopsis thaliana. Biochem J 413, 115-124.

Lou, Y., Gou, J.Y., and Xue, H.W. (2007). PIP5K9, an Arabidopsis phosphatidylinositol monophosphate kinase, interacts with a cytosolic invertase to negatively regulate sugar-mediated root growth. Plant Cell 19, 163-181.

Ma, X., Shor, O., Diminshtein, S., Yu, L., Im, Y.J., Perera, I., Lomax, A., Boss, W.F., and Moran, N. (2009). Phosphatidylinositol $(4,5)$ bisphosphate inhibits K+-efflux channel activity in NT1 tobacco cultured cells. Plant Physiol 149, 1127-1140.

Mahs, A., Ischebeck, T., Heilig, Y., Stenzel, I., Hempel, F., Seiler, S., and Heilmann, I. (2012). The Essential Phosphoinositide Kinase MSS-4 Is Required for Polar Hyphal Morphogenesis, Localizing to Sites of Growth and Cell Fusion in Neurospora crassa. PLoS One 7, e51454.

Mei, Y., Jia, W.J., Chu, Y.J., and Xue, H.W. (2012). Arabidopsis phosphatidylinositol monophosphate 5-kinase 2 is involved in root gravitropism through regulation of polar auxin transport by affecting the cycling of PIN proteins. Cell research 22, 581-597.

Meijer, H.J., and Munnik, T. (2003). Phospholipid-based signaling in plants. Annu Rev Plant Biol 54, 265-306.

Melin, P.M., Pical, C., Jergil, B., and Sommarin, M. (1992). Polyphosphoinositide phospholipase $C$ in wheat root plasma membranes. Partial purification and characterization. Biochim Biophys Acta 1123, 163-169.

Memon, A.R., and Boss, W.F. (1990). Rapid light-induced changes in phosphoinositide kinases and $\mathrm{H}(+)$-ATPase in plasma membrane of sunflower hypocotyls. J Biol Chem 265, 14817-14821.

Molina, H., Horn, D.M., Tang, N., Mathivanan, S., and Pandey, A. (2007). Global proteomic profiling of phosphopeptides using electron transfer dissociation tandem mass spectrometry. Proc Natl Acad Sci U S A 104, 2199-2204. 
Morandell, S., Stasyk, T., Grosstessner-Hain, K., Roitinger, E., Mechtler, K., Bonn, G.K., and Huber, L.A. (2006). Phosphoproteomics strategies for the functional analysis of signal transduction. Proteomics 6, 4047-4056.

Mueller-Roeber, B., and Pical, C. (2002). Inositol phospholipid metabolism in Arabidopsis. Characterized and putative isoforms of inositol phospholipid kinase and phosphoinositide-specific phospholipase C. Plant Physiol 130, 22-46.

Munnik, T., Irvine, R.F., and Musgrave, A. (1998). Phospholipid signalling in plants. Biochim Biophys Acta 1389, 222-272.

Ohlrogge, J., and Browse, J. (1995). Lipid biosynthesis. Plant Cell 7, 957-970.

Ojala, P.J., Paavilainen, V., and Lappalainen, P. (2001). Identification of yeast cofilin residues specific for actin monomer and PIP2 binding. Biochemistry 40, 15562-15569.

Okpodu, C.M., Gross, W., Burkhart, W., and Boss, W.F. (1995). Purification and Characterization of a Soluble Phosphatidylinositol 4-Kinase from Carrot Suspension Culture Cells. Plant Physiol 107, 491-500.

Olsen, J.V., Blagoev, B., Gnad, F., Macek, B., Kumar, C., Mortensen, P., and Mann, M. (2006). Global, in vivo, and site-specific phosphorylation dynamics in signaling networks. Cell 127, 635-648.

Ormo, M., Cubitt, A.B., Kallio, K., Gross, L.A., Tsien, R.Y., and Remington, S.J. (1996). Crystal structure of the Aequorea victoria green fluorescent protein. Science 273, 1392-1395.

Park, S.J., Itoh, T., and Takenawa, T. (2001). Phosphatidylinositol 4-phosphate 5-kinase type I is regulated through phosphorylation response by extracellular stimuli. J Biol Chem 276, 4781-4787.

Perera, I.Y., Heilmann, I., and Boss, W.F. (1999). Transient and sustained increases in inositol 1,4,5-trisphosphate precede the differential growth response in gravistimulated maize pulvini. Proc Natl Acad Sci U S A 96, 5838-5843.

Perera, I.Y., Davis, A.J., Galanopoulou, D., Im, Y.J., and Boss, W.F. (2005). Characterization and comparative analysis of Arabidopsis phosphatidylinositol phosphate 5-kinase 10 reveals differences in Arabidopsis and human phosphatidylinositol phosphate kinases. FEBS letters 579, 3427-3432.

Phillips, S.E., Vincent, P., Rizzieri, K.E., Schaaf, G., Bankaitis, V.A., and Gaucher, E.A. (2006). The diverse biological functions of phosphatidylinositol transfer proteins in eukaryotes. Crit Rev Biochem Mol Biol 41, 21-49.

Pical, C., Westergren, T., Dove, S.K., Larsson, C., and Sommarin, M. (1999). Salinity and hyperosmotic stress induce rapid increases in phosphatidylinositol 4,5-bisphosphate, diacylglycerol pyrophosphate, and phosphatidylcholine in Arabidopsis thaliana cells. J Biol Chem 274, 38232-38240.

Pike, L.J. (1992). Phosphatidylinositol 4-kinases and the role of polyphosphoinositides in cellular regulation. Endocr Rev 13, 692-706.

Preuss, M.L., Schmitz, A.J., Thole, J.M., Bonner, H.K., Otegui, M.S., and Nielsen, E. (2006). A role for the RabA4b effector protein PI-4Kbeta1 in polarized expansion of root hair cells in Arabidopsis thaliana. J Cell Biol 172, 991-998.

Ramachandran, S., Christensen, H.E., Ishimaru, Y., Dong, C.H., Chao-Ming, W., Cleary, A.L., and Chua, N.H. (2000). Profilin plays a role in cell elongation, cell shape maintenance, and flowering in Arabidopsis. Plant Physiol 124, 1637-1647.

Rao, V.D., Misra, S., Boronenkov, I.V., Anderson, R.A., and Hurley, J.H. (1998). Structure of type Ilbeta phosphatidylinositol phosphate kinase: a protein kinase fold flattened for interfacial phosphorylation. Cell 94, 829-839.

Raucher, D., Stauffer, T., Chen, W., Shen, K., Guo, S., York, J.D., Sheetz, M.P., and Meyer, T. (2000). Phosphatidylinositol 4,5-bisphosphate functions as a second messenger that regulates cytoskeleton-plasma membrane adhesion. Cell 100, 221-228.

Read SM, Clarke AE, Bacic A (1993) Stimulation of growth of cultured Nicotiana tabacum W 38 pollen tubes by poly(ethylene glycol) and Cu(II) salts. Protoplasma 177: 1-14 
Reinders, J., and Sickmann, A. (2005). State-of-the-art in phosphoproteomics. Proteomics 5, 4052-4061.

Robert, H.S., and Offringa, R. (2008). Regulation of auxin transport polarity by AGC kinases. Curr Opin Plant Biol 11, 495-502.

Rohacs, T., Lopes, C.M., Michailidis, I., and Logothetis, D.E. (2005). PI(4,5)P2 regulates the activation and desensitization of TRPM8 channels through the TRP domain. Nat Neurosci 8, 626-634.

Rozelle, A.L., Machesky, L.M., Yamamoto, M., Driessens, M.H., Insall, R.H., Roth, M.G., LubyPhelps, K., Marriott, G., Hall, A., and Yin, H.L. (2000). Phosphatidylinositol 4,5bisphosphate induces actin-based movement of raft-enriched vesicles through WASPArp2/3. Curr Biol 10, 311-320.

Rudge, S.A., Anderson, D.M., and Emr, S.D. (2004). Vacuole size control: regulation of PtdIns(3,5)P2 levels by the vacuole-associated Vac14-Fig4 complex, a PtdIns(3,5)P2specific phosphatase. Mol Biol Cell 15, 24-36.

Santarius, M., Lee, C.H., and Anderson, R.A. (2006). Supervised membrane swimming: small G-protein lifeguards regulate PIPK signalling and monitor intracellular Ptdlns(4,5)P2 pools. Biochem J 398, 1-13.

Schmid, A.C., Wise, H.M., Mitchell, C.A., Nussbaum, R., and Woscholski, R. (2004). Type II phosphoinositide 5-phosphatases have unique sensitivities towards fatty acid composition and head group phosphorylation. FEBS letters 576, 9-13.

Shevchenko, A., Wilm, M., Vorm, O., and Mann, M. (1996). Mass spectrometric sequencing of proteins silver-stained polyacrylamide gels. Analytical chemistry 68, 850-858.

Shibasaki, Y., Ishihara, H., Kizuki, N., Asano, T., Oka, Y., and Yazaki, Y. (1997). Massive actin polymerization induced by phosphatidylinositol-4-phosphate 5-kinase in vivo. J Biol Chem 272, 7578-7581.

Shimomura, O., Johnson, F.H., and Saiga, Y. (1962). Extraction, purification and properties of aequorin, a bioluminescent protein from the luminous hydromedusan, Aequorea. J Cell Comp Physiol 59, 223-239.

Singer, S.J., and Nicholson, G.L. (1972). The fluid mosaic model of the structure of cell membranes. Science 175.

Sohn, R.H., Chen, J., Koblan, K.S., Bray, P.F., and Goldschmidt-Clermont, P.J. (1995). Localization of a binding site for phosphatidylinositol 4,5-bisphosphate on human profilin. J Biol Chem 270, 21114-21120.

Somerville, C., Browse, J., Jaworski, J.G., and Ohlrogge, J.B. (2000). Lipids. In Biochemistry and Molecular Biology of Plants, B.B. Buchanan, W. Gruissem, and R.L. Jones, eds (Rockville, MD: American Society of Plant Physiologists), pp. 456-526.

Sousa, E., Kost, B., and Malho, R. (2008). Arabidopsis Phosphatidylinositol-4-Monophosphate 5-Kinase 4 Regulates Pollen Tube Growth and Polarity by Modulating Membrane Recycling. Plant Cell 20, 3050-3064.

Sperling, P., and Heinz, E. (2003). Plant sphingolipids: structural diversity, biosynthesis, first genes and functions. Biochim Biophys Acta 1632, 1-15.

Starling, A.P., East, J.M., and Lee, A.G. (1995). Phosphatidylinositol 4-phosphate increases the rate of dephosphorylation of the phosphorylated $\mathrm{Ca}(2+)$-ATPase. J Biol Chem 270, 14467-14470.

Stenzel, I., Ischebeck, T., Quint, M., and Heilmann, I. (2012). Variable regions of PI4P 5-kinases direct PtdIns(4,5)P2 toward alternative regulatory functions in tobacco pollen tubes. Front Plant Sci 2, 1-14.

Stenzel, I., Ischebeck, T., König, S., Holubowska, A., Sporysz, M., Hause, B., and Heilmann, I. (2008). The type B phosphatidylinositol-4-phosphate 5-kinase 3 is essential for root hair formation in Arabidopsis thaliana. Plant Cell 20, 124-141. 
Stevenson-Paulik, J., Love, J., and Boss, W.F. (2003). Differential regulation of two Arabidopsis type III phosphatidylinositol 4-kinase isoforms. A regulatory role for the pleckstrin homology domain. Plant Physiol 132, 1053-1064.

Stevenson, J.M., Perera, I.Y., and Boss, W.F. (1998). A phosphatidylinositol 4-kinase pleckstrin homology domain that binds phosphatidylinositol 4-monophosphate. J Biol Chem 273, 22761-22767.

Stevenson, J.M., Perera, I.Y., Heilmann, I., Persson, S., and Boss, W.F. (2000). Inositol signaling and plant growth. Trends Plant Sci 5, 252-258.

Strahl, T., and Thorner, J. (2007). Synthesis and function of membrane phosphoinositides in budding yeast, Saccharomyces cerevisiae. Biochim Biophys Acta 1771, 353-404.

Sugiyama, N., Nakagami, H., Mochida, K., Daudi, A., Tomita, M., Shirasu, K., and Ishihama, Y. (2008). Large-scale phosphorylation mapping reveals the extent of tyrosine phosphorylation in Arabidopsis. Mol Syst Biol 4, 193.

Suh, B.C., and Hille, B. (2008). PIP2 is a necessary cofactor for ion channel function: how and why? Annu Rev Biophys 37, 175-195.

Takeshima, H., Komazaki, S., Nishi, M., lino, M., and Kangawa, K. (2000). Junctophilins: a novel family of junctional membrane complex proteins. Molecular cell 6, 11-22.

Tang, G.Q., Hardin, S.C., Dewey, R., and Huber, S.C. (2003). A novel C-terminal proteolytic processing of cytosolic pyruvate kinase, its phosphorylation and degradation by the proteasome in developing soybean seeds. Plant J 34, 77-93.

Tang, G.Q., Novitzky, W.P., Carol Griffin, H., Huber, S.C., and Dewey, R.E. (2005). Oleate desaturase enzymes of soybean: evidence of regulation through differential stability and phosphorylation. Plant J 44, 433-446.

Thiele, A., Weiwad, M., Zerweck, J., Fischer, G., and Schutkowski, M. (2010). High density peptide microarrays for proteome-wide fingerprinting of kinase activities in cell lysates. Methods Mol Biol 669, 173-181.

Thingholm, T.E., Jensen, O.N., and Larsen, M.R. (2009). Analytical strategies for phosphoproteomics. Proteomics 9, 1451-1468.

Thole, J.M., Vermeer, J.E., Zhang, Y., Gadella, T.W., Jr., and Nielsen, E. (2008). ROOT HAIR DEFECTIVE4 encodes a phosphatidylinositol-4-phosphate phosphatase required for proper root hair development in Arabidopsis thaliana. Plant Cell 20, 381-395.

Turkish, A.R., and Sturley, S.L. (2009). The genetics of neutral lipid biosynthesis: an evolutionary perspective. American journal of physiology. Endocrinology and metabolism 297, E19-27.

Vancurova, I., Choi, J.H., Lin, H., Kuret, J., and Vancura, A. (1999). Regulation of phosphatidylinositol 4-phosphate 5-kinase from Schizosaccharomyces pombe by casein kinase I. J Biol Chem 274, 1147-1155.

Vanhaesebroeck, B., Leevers, S.J., Ahmadi, K., Timms, J., Katso, R., Driscoll, P.C., Woscholski, R., Parker, P.J., and Waterfield, M.D. (2001). Synthesis and function of 3phosphorylated inositol lipids. Annu Rev Biochem 70, 535-602.

Varsanyi, M., Tolle, H.G., Heilmeyer, M.G., Jr., Dawson, R.M., and Irvine, R.F. (1983). Activation of sarcoplasmic reticular Ca2+ transport ATPase by phosphorylation of an associated phosphatidylinositol. Embo J 2, 1543-1548.

Vincent, P., Chua, M., Nogue, F., Fairbrother, A., Mekeel, H., Xu, Y., Allen, N., Bibikova, T.N., Gilroy, S., and Bankaitis, V.A. (2005). A Sec14p-nodulin domain phosphatidylinositol transfer protein polarizes membrane growth of Arabidopsis thaliana root hairs. J Cell Biol 168, 801-812.

Vollhardt, K. P. C., Schore, N. E., Peter, K. (2005). Organische Chemie. Wiley-VCH.

Wasteneys, G.O., and Galway, M.E. (2003). Remodeling the cytoskeleton for growth and form: an overview with some new views. Annu Rev Plant Biol 54, 691-722.

Wasteneys, G.O., and Yang, Z. (2004). New views on the plant cytoskeleton. Plant Physiol 136, 3884-3891. 
Westergren, T., Dove, S.K., Sommarin, M., and Pical, C. (2001). AtPIP5K1, an Arabidopsis thaliana phosphatidylinositol phosphate kinase, synthesizes Ptdlns $(3,4) \mathrm{P}(2)$ and Ptdlns $(4,5) \mathrm{P}(2)$ in vitro and is inhibited by phosphorylation. Biochem J 359, 583-589.

Whatmore, J., Morgan, C.P., Cunningham, E., Collison, K.S., Willison, K.R., and Cockcroft, S. (1996). ADP-ribosylation factor 1-regulated phospholipase D activity is localized at the plasma membrane and intracellular organelles in HL60 cells. Biochem J 320 ( Pt 3), 785-794.

Witke, W. (2004). The role of profilin complexes in cell motility and other cellular processes. Trends Cell Biol 14, 461-469.

Xue, H.W., Pical, C., Brearley, C., Elge, S., and Muller-Rober, B. (1999). A plant 126-kDa phosphatidylinositol 4-kinase with a novel repeat structure. Cloning and functional expression in baculovirus-infected insect cells. J Biol Chem 274, 5738-5745.

Yamamoto, M., Hilgemann, D.H., Feng, S., Bito, H., Ishihara, H., Shibasaki, Y., and Yin, H.L. (2001). Phosphatidylinositol 4,5-bisphosphate induces actin stress-fiber formation and inhibits membrane ruffling in CV1 cells. J Cell Biol 152, 867-876.

Yang, X.J., and Seto, E. (2008). Lysine acetylation: codified crosstalk with other posttranslational modifications. Molecular cell 31, 449-461.

Yin, H.L., and Janmey, P.A. (2003). Phosphoinositide regulation of the actin cytoskeleton. Annu Rev Physiol 65, 761-789.

Zhao, Y., Yan, A., Feijo, J.A., Furutani, M., Takenawa, T., Hwang, I., Fu, Y., and Yang, Z. (2010). Phosphoinositides regulate clathrin-dependent endocytosis at the tip of pollen tubes in Arabidopsis and tobacco. Plant Cell 22, 4031-4044.

Zimmermann, P., Hirsch-Hoffmann, M., Hennig, L., and Gruissem, W. (2004). GENEVESTIGATOR. Arabidopsis microarray database and analysis toolbox. Plant Physiol 136, 2621-2632. 


\section{APPENDIX}

\section{Table 7.1. List of the oligonucleotides used in this thesis}

\begin{tabular}{|c|c|c|}
\hline No. & Oligonucleotide sequence & Purpose \\
\hline P1 & 5'-actaaagggcaataatccttccacc-3' & $\begin{array}{l}\text { Identification of T-DNA insertion } \\
\text { mutants, PIP5K1 for }\end{array}$ \\
\hline P2 & $5^{\prime}$-gcaaattctcatggccaagtgga-3' & $\begin{array}{l}\text { Identification of T-DNA insertion } \\
\text { mutants, PIP5K1 rev }\end{array}$ \\
\hline P3 & 5'-caggtttgatacaatgcacaccat-3' & $\begin{array}{l}\text { Identification of T-DNA insertion } \\
\text { mutants, PIP5K2 for }\end{array}$ \\
\hline P4 & $5^{\prime}$-tgggagtctgatggagaagctg-3' & $\begin{array}{l}\text { Identification of T-DNA insertion } \\
\text { mutants, PIP5K2 rev }\end{array}$ \\
\hline P5 & 5'-gtaggtattatagatcgttagcttg-3' & $\begin{array}{l}\text { Identification of T-DNA insertion } \\
\text { mutants, PIP5K3 for }\end{array}$ \\
\hline P6 & $5^{\prime}$-tatcccaactaggtcttggattct-3' & $\begin{array}{l}\text { Identification of T-DNA insertion } \\
\text { mutants, PIP5K3 rev }\end{array}$ \\
\hline P7 & $5^{\prime}$-tggttcacgtagtgggccatcg-3' & $\begin{array}{l}\text { Identification of T-DNA insertion } \\
\text { mutants, LBa1SALK for }\end{array}$ \\
\hline P8 & $5^{\prime}$-atggtgagcaagggcgaggag $-3^{\prime}$ & $\begin{array}{l}\text { Identification of T-DNA insertion } \\
\text { mutants, PIP5K3 end for }\end{array}$ \\
\hline P9 & $5^{\prime}$-attaccatcttgcagattggc -3 & $\begin{array}{l}\text { Identification of T-DNA insertion } \\
\text { mutants, EYFP rev }\end{array}$ \\
\hline P10 & 5'-gcatggaattaggtacgctgtcggcaaacacgc-3' & $\begin{array}{l}\text { QuickChange site-directed } \\
\text { mutagenesis, PIPK2 S370A for }\end{array}$ \\
\hline P 11 & 5'-gcgtgtttgccgacagcgtacctaattccatgc-3' & $\begin{array}{l}\text { QuickChange site-directed } \\
\text { mutagenesis, PIPK2 S370A rev }\end{array}$ \\
\hline P 12 & 5'-gcatggaattaggtacgatgtcggcaaacacgc-3' & $\begin{array}{l}\text { QuickChange site-directed } \\
\text { mutagenesis, PIPK2 S370D for }\end{array}$ \\
\hline P 13 & $5^{\prime}$-gcgtgtttgccgacatcgtacctaattccatgc-3' & $\begin{array}{l}\text { QuickChange site-directed } \\
\text { mutagenesis, PIPK2 S370D rev }\end{array}$ \\
\hline P 14 & $5^{\prime}$-cacaccaccgcatctagctgtggatttccgctgg-3' & $\begin{array}{l}\text { QuickChange site-directed } \\
\text { mutagenesis, PIPK2 S409A for }\end{array}$ \\
\hline P 15 & $5^{\prime}$-ccagcggaaatccacagctagatgcggtggtgtg-3' & $\begin{array}{l}\text { QuickChange site-directed } \\
\text { mutagenesis, PIPK2 S409A rev }\end{array}$ \\
\hline P 16 & $5^{\prime}$-cacaccaccgcatctagatgtggatttccgctgg-3' & $\begin{array}{l}\text { QuickChange site-directed } \\
\text { mutagenesis, PIPK2 S409D for }\end{array}$ \\
\hline P17 & $5^{\prime}$-ccagcggaaatccacatctagatgcggtggtgtg-3' & $\begin{array}{l}\text { QuickChange site-directed } \\
\text { mutagenesis, PIPK2 S409D rev }\end{array}$ \\
\hline P 18 & $5^{\prime}$-caagacggtgaagaaagctgaagtcaaggtgcttc- $3^{\prime}$ & $\begin{array}{l}\text { QuickChange site-directed } \\
\text { mutagenesis, PIPK2 S475A for }\end{array}$ \\
\hline P 19 & $5^{\prime}$-gaagcaccttgacttcagctttcttcaccgtcttg-3' & $\begin{array}{l}\text { QuickChange site-directed } \\
\text { mutagenesis, PIPK2 S475A rev }\end{array}$ \\
\hline P 20 & $5^{\prime}$-caagacggtgaagaaagatgaagtcaaggtgcttc-3' & $\begin{array}{l}\text { QuickChange site-directed } \\
\text { mutagenesis, PIPK2 S475D for }\end{array}$ \\
\hline P 21 & 5'-gaagcaccttgacttcatctttcttcaccgtcttg-3' & $\begin{array}{l}\text { QuickChange site-directed } \\
\text { mutagenesis, PIPK2 S475D rev }\end{array}$ \\
\hline
\end{tabular}




\section{Table 7.1. List of the oligonucleotides used in this thesis (continued)}

\begin{tabular}{|c|c|c|}
\hline P 22 & 5'-gaaaatacacttgtggctaggttctatggtgtgc-3' & $\begin{array}{l}\text { QuickChange site-directed } \\
\text { mutagenesis, PIPK2 T500A for }\end{array}$ \\
\hline P 23 & 5'-gcacaccatagaacctagccacaagtgtattttc-3' & $\begin{array}{l}\text { QuickChange site-directed } \\
\text { mutagenesis, PIPK } 2 \text { T500A rev }\end{array}$ \\
\hline P 24 & 5'-cagtatgaaaatacacttgtggataggttctatggtgtgc-3' & $\begin{array}{l}\text { QuickChange site-directed } \\
\text { mutagenesis, PIPK2 T500A for }\end{array}$ \\
\hline P 25 & $5^{\prime}$-gcacaccatagaacctatccacaagtgtattttcatactg-3' & $\begin{array}{l}\text { QuickChange site-directed } \\
\text { mutagenesis, PIPK2 T500A rev }\end{array}$ \\
\hline P 26 & $5^{\prime}$-gaccttaaagggtctgcccatggacggtatacc-3' & $\begin{array}{l}\text { QuickChange site-directed } \\
\text { mutagenesis, PIPK2 S541A for }\end{array}$ \\
\hline P 27 & 5'-ggtataccgtccatgggcagaccctttaaggtc-3' & $\begin{array}{l}\text { QuickChange site-directed } \\
\text { mutagenesis, PIPK2 S541A rev }\end{array}$ \\
\hline P 28 & 5'-gaccttaaagggtctgaccatggacggtatacc- $3^{\prime}$ & $\begin{array}{l}\text { QuickChange site-directed } \\
\text { mutagenesis, PIPK2 S541D for }\end{array}$ \\
\hline P 29 & 5'-ggtataccgtccatggtcagaccctttaaggtc-3' & $\begin{array}{l}\text { QuickChange site-directed } \\
\text { mutagenesis, PIPK2 S541D rev }\end{array}$ \\
\hline P 30 & $5^{\prime}$-cccatggacggtatgcctccaaacctgaagg-3' & $\begin{array}{l}\text { QuickChange site-directed } \\
\text { mutagenesis, PIPK2 T546A for }\end{array}$ \\
\hline P 31 & 5'-ccttcaggtttggaggcataccgtccatggg-3' & $\begin{array}{l}\text { QuickChange site-directed } \\
\text { mutagenesis, PIPK2 T546A rev }\end{array}$ \\
\hline P 32 & 5'-cccatggacggtatgactccaaacctgaagg-3' & $\begin{array}{l}\text { QuickChange site-directed } \\
\text { mutagenesis, PIPK2 T546D for }\end{array}$ \\
\hline P 33 & 5'-ccttcaggtttggagtcataccgtccatggg-3' & $\begin{array}{l}\text { QuickChange site-directed } \\
\text { mutagenesis, PIPK2 T546D rev }\end{array}$ \\
\hline P 34 & $5^{\prime}$-caagaactctttagtcgccaagttctttggggtgc-3' & $\begin{array}{l}\text { QuickChange site-directed } \\
\text { mutagenesis, PIPK3 T472A for }\end{array}$ \\
\hline P 35 & 5'-gcaccccaaagaacttggcgactaaagagttcttg-3' & $\begin{array}{l}\text { QuickChange site-directed } \\
\text { mutagenesis, PIPK3 T472A rev }\end{array}$ \\
\hline P 36 & 5'-caagaactctttagtcgacaagttctttggggtgc-3' & $\begin{array}{l}\text { QuickChange site-directed } \\
\text { mutagenesis, PIPK3 T472D for }\end{array}$ \\
\hline P 37 & 5'-gcaccccaaagaacttgtcgactaaagagttcttg-3' & $\begin{array}{l}\text { QuickChange site-directed } \\
\text { mutagenesis, PIPK3 T472D rev }\end{array}$ \\
\hline
\end{tabular}


A



C



B

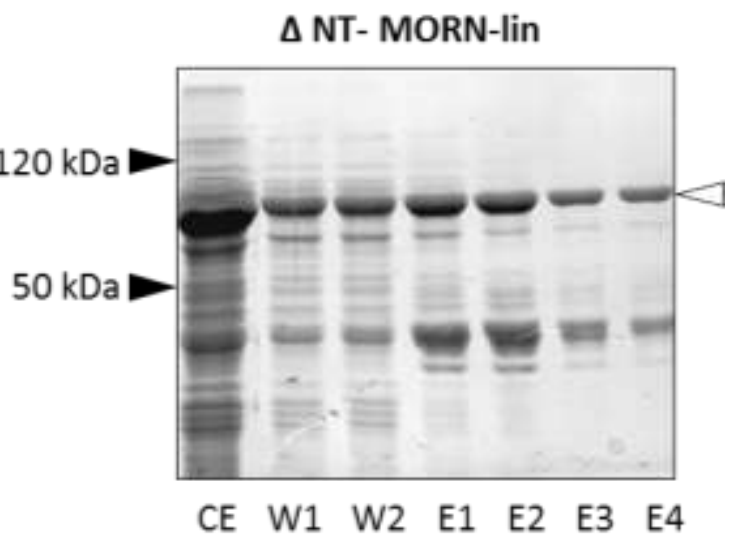

Fig. 7.1: Purification of truncated MBP-PIP5K2 versions. PIP5K2 truncations were recombinantly expressed in E. coli and enriched using affinity chromatography. CE, crude extract; W, wash; E, eluted fraction. White arrowheads mark the size of the respective truncation. Black arrowheads indicate protein sizes. NT, N-terminal domain; MORN, membrane-occupation-and-recognition-nexus domain; Lin, Linker domain; Cat, catalytic domain. 



Fig. 7.2: Spectra of phosphorylated peptides identified with MS-analyses. Recombinant MBP-PIP5K2 was phosphorylated with leaf extract and proteins were separated by SDS-PAGE. The coomassie-stained MBP-PIP5K2 band was tryptic digested and peptides were analysed with MS. Figure shows the respective spectra. $A$, spectrum of the corresponding peptide for phosphorylation site T430 (ELFTVDPADYLAICGNDALR); B, spectrum of the corresponding peptide for phosphorylation site S598 (IMDYSLLVGVHFR). 
1.



2.

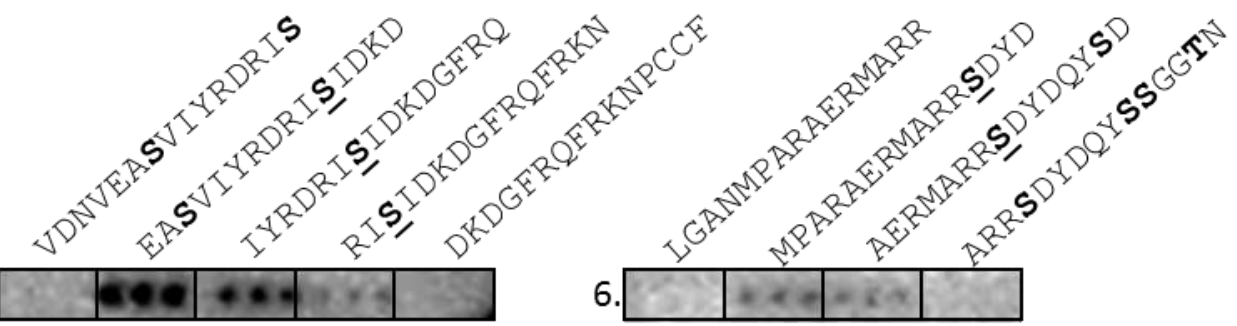

3.

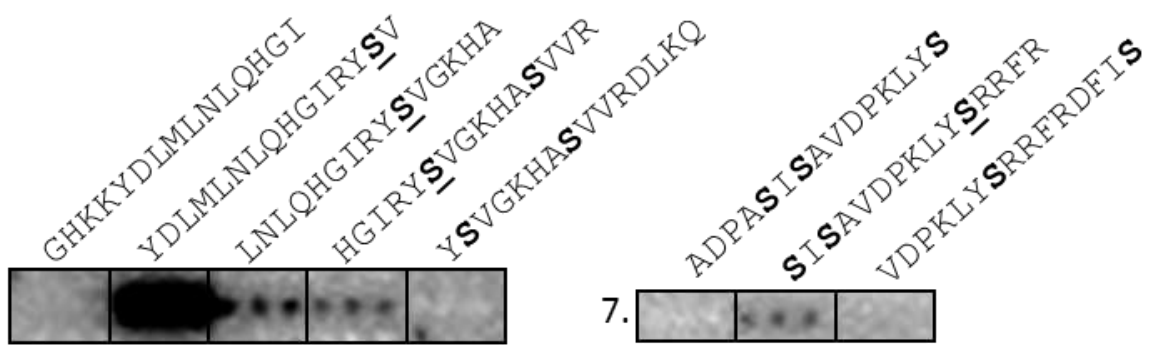

4.

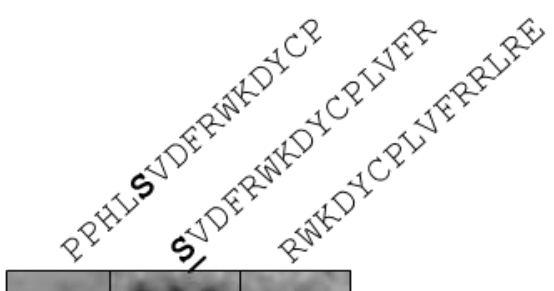

Fig. 7.3: Phosphorylated peptides determined with peptide array analyses. Phosphorylated peptides were identified by using a peptide map supplemented with the peptide array kit. Possible phosphorylation sites are shown in bold letters. By comparing phosphorylated peptides with not phosphorylated neighbour peptides phosphorylation sites can be estimated (bold underlined letters). 1, phosphorylation sites: T270, S274, S275; 2, phosphorylation sites: S323; 3, phosphorylation sites: S370; 4, phosphorylation sites: S409; 5, phosphorylation sites: S455, S457, S462; 6, phosphorylation sites: S676; 7, S739. 


\subsection{List of abbreviations}

\begin{tabular}{|c|c|}
\hline Agrobac. tumefaciens & Agrobacterium tumefaciens \\
\hline$A m p^{R}$ & Ampicillin \\
\hline ATP & Adenosine trisphosphate \\
\hline ADP & Adenosine diphosphate \\
\hline AP & Alkaline phosphatase \\
\hline APS & ammonium persulfate \\
\hline BASTA & Glufosinate-Ammonium \\
\hline $\mathrm{CaCl}_{2}$ & Calcium chloride \\
\hline Cat & Catalytic domain \\
\hline cDNA & Complementary desoxyribonucleic acid \\
\hline CTAB & Cetyltrimethylammoniumbromid \\
\hline $\mathrm{CuSO}_{4}$ & Copper sulfate \\
\hline DAG & Diacyl glycerol \\
\hline Dim & Dimerization domain \\
\hline DGDG & Digalactosyldiacylglycerol \\
\hline $\mathrm{dd} \mathrm{H}_{2} \mathrm{O}$ & Double distilled water \\
\hline DMF & Dimethylformamid \\
\hline DMSO & dimethyl sulfoxide \\
\hline DNA & Desoxyribonucleic acid \\
\hline dNTPs & Desoxyribonucleotide \\
\hline DTT & dithiothreitol \\
\hline E. coli & Escherichia coli \\
\hline EDTA & Ethylene diamine tetra acetate \\
\hline EGTA & ethylene glycol tetraacetic acid \\
\hline $\mathrm{ER}$ & Endoplasmic recticulum \\
\hline EYFP & Enhanced yellow fluorescent protein \\
\hline $\mathrm{H}_{3} \mathrm{BO}_{3}$ & Boric acid \\
\hline $\mathrm{HCl}$ & Hydrochloric acid \\
\hline HEPES & 4-(2-hydroxyethyl)-1-piperazineethanesulfonic acid \\
\hline InsP 3 & Inositol 3-phosphate \\
\hline IPTG & Isopropyl- $\beta$-D-thiogalactosylpyranosid \\
\hline $\operatorname{Kan}^{\mathrm{R}}$ & Kanamycin \\
\hline $\mathrm{KCl}$ & Potassium chloride \\
\hline
\end{tabular}




\begin{tabular}{|c|c|}
\hline $\mathrm{KOH}$ & Potassium hydroxide \\
\hline Lin & Linker domain \\
\hline LC/MS & $\begin{array}{l}\text { Reversed-Phase High Performance Liquid Chromatography coupled to } \\
\text { mass spectrometry }\end{array}$ \\
\hline $\mathrm{MgCl}_{2}$ & Magnesium chlorite \\
\hline MGDG & Monogalactosyldiacylglycerol \\
\hline $\mathrm{MgSO}_{4}$ & Magnesium sulphate \\
\hline $\mathrm{MnCl}_{2}$ & Manganese (II)-chloride \\
\hline MORN & Membrane occupation aand recognition nexus-domain \\
\hline MBP & Maltose binding protein \\
\hline MS & Mass spectrometry \\
\hline $\mathrm{Na}_{2} \mathrm{MoO}_{4}$ & Sodium molybdate \\
\hline $\mathrm{NaCl}$ & Sodium chlorite \\
\hline $\mathrm{NaOH}$ & Sodium hydroxide \\
\hline $\mathrm{NaF}$ & Sodium fluoride \\
\hline $\mathrm{Na}_{3} \mathrm{VO}_{4}$ & Sodium orthovanadate \\
\hline NBT & nitro blue tetrazolium chloride \\
\hline $\mathrm{NH}_{4} \mathrm{OH}$ & Ammonium hydroxide \\
\hline $\mathrm{NH}_{4} \mathrm{HCO}_{3}$ & Ammonium bicarbonate \\
\hline PCR & Polymerase chain reaction \\
\hline $\mathrm{PI}$ & Phosphoinositide \\
\hline Pl4-kinases & Ptdlns 4-kinases \\
\hline PIP5-kinases & PtdIns4P 5-kinases \\
\hline PI-PLCs & PI-specific PLCs \\
\hline PIPES & piperazine-N,N'-bis(2-ethanesulfonic acid) \\
\hline PLC & Phospholipase C \\
\hline $\mathrm{PI3K}$ & Phosphoinositide 3-kinase \\
\hline $\mathrm{PI} 4 \mathrm{~K}$ & Phosphoinositide 4-kinase \\
\hline PKA & Protein kinase $A$ \\
\hline PMSF & phenylmethanesulfonylfluoride \\
\hline Ptdlns & Phosphatidylinositol \\
\hline Ptdlns4,5P 2 & Phosphatidylinositol 4,5-bisphosphate \\
\hline Ptdlns4P & Phosphatidylinositol 4-phosphate \\
\hline PIPKs & Phosphatidylinositol-bisphosphate-kinase \\
\hline PIS & Phosphatidylinositol-synthase \\
\hline
\end{tabular}




$\begin{array}{ll}\text { PtdCho } & \text { Phosphatidylcholine } \\ \text { PtdEtn } & \text { Phosphatidylethanolamine } \\ \text { PtdOH } & \text { Phosphatidic acid } \\ \text { PtdSer } & \text { Phosphatidylserine } \\ \text { SAP } & \text { Shrimp alkaline phosphatase } \\ \text { SDS } & \text { Sodium dodecyl phosphate } \\ \text { TAG } & \text { Triacylglycerol } \\ \text { TEMED } & \text { Tetramethylethylenediamine } \\ \text { TEV } & \text { Tobacco etch virus } \\ \text { TFA } & \text { trifluoroacetic acid } \\ \text { TRIS } & \text { Tris(hydroxymethyl)aminomethane } \\ \text { X-phosphate } & \text { 5-bromo-4-chloro-3-indolyl phosphate }\end{array}$




\section{ACKNOWLEDGMENTS - DANKSAGUNG}

An erster Stelle möchte ich mich bei meinem Doktorvater Prof. Dr. Ingo Heilmann für das interessante Forschungsprojekt und die motivierende und umfassende Betreuung bedanken. Vielen Dank für die vielen anregenden und lehrreichen Diskussionen; für die Unterstützung bei der Umsetzung eigener Ideen; für die beruhigenden Worte vor und nach Vorträgen (oder radioaktiven Experimenten); für die Möglichkeit, auf so vielen Tagungen in der ganzen Welt, meine Daten vorstellen zu können und nicht zuletzt für die Fröhlichkeit, die mich in den letzten dreieinhalb Jahren gerne zur Arbeit kommen ließ.

Bei PD Dr. Thomas Teichmann möchte ich mich ganz herzlich für die Übernahme des Korreferates bedanken.

Meinem Thesis Komitee bestehend aus Prof. Dr. Ingo Heilmann, Prof. Dr. Andrea Polle und Prof. Dr. Steven Johnsen, danke ich für das Interesse an meiner Arbeit, die guten Ratschläge und anregenden Diskussionen.

Bei Prof. Dr. Ivo Feußner, Prof. Dr. Gregor Bucher und PD Dr. Thomas Teichmann bedanke ich mich für die Bereitschaft, zusammen mit meinem Thesis Komitee meine Prüfungskommission zu bilden. Bei Prof. Dr. Ivo Feußner möchte ich mich insbesondere für die Möglichkeit bedanken, in seiner Abteilung die ersten Jahre meiner Doktorarbeit absolvieren zu können und die hervorragende Ausstattung nutzen zu dürfen, sowie für interessante und konstruktive Diskussionen.

Der Göttinger Graduiertenschule für Neurowissenschaften und Molekulare Biowissenschaften (GGNB) danke ich für das interessante Angebot an Methodenkursen, Soft-Skill-Kursen und Freizeitaktivitäten sowie besonders für die Finanzierung meiner Promotion in Form zweier 3monatiger Brückenstipendien und die Finanzierung allerlei Tagungen. Zudem möchte ich dem Dorothea Schlözer-Programm für die 2-jährige Finanzierung meiner Promotion und die Möglichkeit zur Vernetzung mit zahlreichen anderen Wissenschaftlerinnen danken.

Ganz besonders möchte ich mich auch bei Dr. Waltraud Schulze und der AG Baginsky für das Messen meiner MS-Proben, sowie Prof. Dr. Mike Schutkowski für die Hilfe beim Peptide Array und Dr. Nico Dissmeyer für die hilfreichen Tipps rund um Proteinkinasen bedanken.

Bedanken möchte ich mich desweiteren bei PD Dr. Margret Köck für die Möglichkeit, das Isotopenlabor ihrer Abteilung nutzen zu dürfen. Ein großes Dankeschön geht in diesem Zusammenhang auch an Karin Klar.

Der AG Behrens der Uni Halle sei gedankt für die Möglichkeit, das konfokale Mikroskop nutzen zu können. 
Bei unseren Gärtnerinnen Susanne Mester (Uni Göttingen) und Frau Rosinsky (Uni Halle) bedanke ich mich für die gewissenhafte Pflege meiner Pflanzen und die vielen Anzuchtstipps auch für das heimische Grün.

Dr. Ellen Hornung danke ich für den pCAMBIA-Vektor, ihr offenes Ohr und ihre stete Hilfsbereitschaft.

Bedanken möchte ich mich auch bei Dr. Till Ischebeck und Dr. Irene Stenzel für die Versorgung mit transgenem Saatgut, allerlei Vektoren und die vielen hilfreichen Gedankenanstöße.

Bei Dr. Mareike Heilmann möchte ich mich ganz herzlich für die umfangreiche Rund-umVersorgung mit wissenschaftlichen Tipps, Tricks und Anregungen.

Ein besonderes Dankeschön geht auch an Franziska Hempel für die zahlreichen, teils auch hitzigen, wissenschaftlichen Diskussionen, die Unterstützung beim Studieren, Experimentieren und beim Englischlernen.

Bei meiner Praktikantin Sophie Narstedt und meinem Bachelor Lutz Bocher bedanke ich mich für ihre fleißige Mitarbeit.

Desweiteren bedanke ich mich bei der Abteilung Biochemie der Pflanze aus Göttingen für die angenehme Arbeitsatmosphäre und die vielen fröhlichen Wanderungen.

Ein großes Dankeschön gilt der AG Heilmann, den ehemaligen Mitgliedern, den neuen Mitgliedern und den ständigen Mitgliedern. Danke an: Alina, für ihren großartigen Musikgeschmack; Anette, für ihre grenzenlose Ruhe; Till, für die spannenden Kickerduelle; Irene, für die Kamera-Gespräche; Mareike, für den Humor, der genau meinen Lachnerv trifft; Ingo, für die klassisch humanistische Bildung; Katha und Anna, für ihr fröhliches Gemüt; Ralitza, für ihr lautes, ansteckendes Lachen; Tobi und Lars, für die Aufrechterhaltung der MännerQuote; Johanna, für die leckeren Backkünste; Marion, für die Hilfsbereitschaft, und nicht zuletzt vor allem Steffi (höhl) und Franzi, meine beiden Mitdoktorandinnen, für die Unterstützung und das viele Lachen in den letzten Jahren.

Bedanken möchte ich mich auch bei Julia und Urs, für die lustigen und oftmals nerdigen Abendbrotgespräche, für das Kochen und Einkaufen während meiner Schreibphase und für die vielfältigen Ablenkungsmaßnahmen vom Arbeitsalltag.

Zuletzt möchte ich mich bei meiner Familie, insbesondere bei meinen Eltern, bedanken. Vielen Dank für den Rückhalt, die Anerkennung, die Ermutigung und vor allem die Möglichkeit, genau das studieren zu können, was mir Spaß macht. 


\section{CURRICULUM VItAE}

$\begin{array}{ll}\text { Name } & \text { Jennifer Lerche } \\ \text { Address } & \text { Department of Cellular Biochemistry } \\ \text { Institute for Biochemistry and Biotechnology } & \text { Martin-Luther-University Halle Wittenberg } \\ & \text { Kurt-Mothes Straße 3, 06120 Halle (Saale), Germany } \\ & \\ \text { Phone } & \text { Jennifer.lerche@biochemtech.uni-halle.de } \\ \text { Home address } & \\ \text { Email } & \text { German } \\ \text { Nationality } & \text { unmarried, no children } \\ \text { Status } & \text { July 3 }{ }^{\text {rd }}, 1985, \text { in Bad Gandersheim, Germany } \\ \text { Date and place of birth }\end{array}$

\section{Scientific Education and Qualifications}

\section{Studies}

2009-2013

Doctoral thesis at the Albrecht-von-Haller-Institute for Plant Sciences (Georg August University Göttingen) and at the Institute for Biochemistry and Biotechnology (Martin Luther University HalleWittenberg) (with Prof. Dr. Ingo Heilmann)

Thesis title: "Control of PI4P 5-kinases by reversible phosphorylation in Arabidopsis thaliana"

since 2009

Accepted to the Göttingen Graduate School for Neurosciences and Molecular Biosciences (GGNB), an interdisciplinary training platform for excellent doctoral students in neurosciences and molecular biosciences

Diploma thesis at the Albrecht-von-Haller-Institute for Plant Sciences (with Prof. Dr. Ivo Feussner/Prof. Dr. Ingo Heilmann)

Thesis title: "Constitutive and stress-induced PI4P 5-kinases in Arabidopsis thaliana" (in German; grade "very good" / 1.0) 
$2004-2009$

Studies of Biology at Georg August University Göttingen;

Degree: Diploma (grade "very good" / 1.3)

Major subject: Biochemistry

Minor subjects: Microbiology and Organical Chemistry

\section{Presentations at International Conferences}

2011

Poster at the $24^{\text {th }}$ Conference of Plant Molecular Biology

(Dabringhausen, Germany)

Poster at the $2^{\text {nd }}$ International Meeting "Conformational Transitions

in Macromolecular Interactions" (Halle, Germany)

Poster at the Plant Science Student Conference (Halle, Germany)

Poster at the EuroFed Lipid conference (Gdansk, Poland)

2010

Poster at the $19^{\text {th }}$ International Symposium on Plant Lipids (Cairns,

Australia)

\section{Scholarships}

2012-2013

GGNB Bridging Fund to finalize Ph.D. studies

$2009-2012$

Scholar ship of the Dorothea Schlözer Fellowship Programme (Georg August University Göttingen)

2009

GGNB Bridging Fund to start Ph.D. studies

\section{Publications}

König, S., Ischebeck, T., Lerche, J., Stenzel, I., and Heilmann, I. (2008). Salt stress-induced association of phosphatidylinositol-4,5-bisphosphate with clathrin-coated vesicles in plants. Biochem J 415, 387-399.

Ternes, P., Feussner, K., Werner, S., Lerche, J., Iven, T., Heilmann, I., Riezman, H., Feussner, I. (2011) Disruption of the ceramide synthase LOH1 causes spontaneous cell death in Arabidopsis thaliana. New Phytol, 192(4), 841-854.

Saavedra L, Balbi V, Lerche J, Mikami K, Heilmann I, Sommarin M. (2011). PIPKs are essential for rhizoid elongation and caulonemal cell development in the moss Physcometrella patens. Plant J,67(4), 635-647. 